\title{
Inaugural-Dissertation
}

Essays on Inequality, Economic Growth and Political Polarization

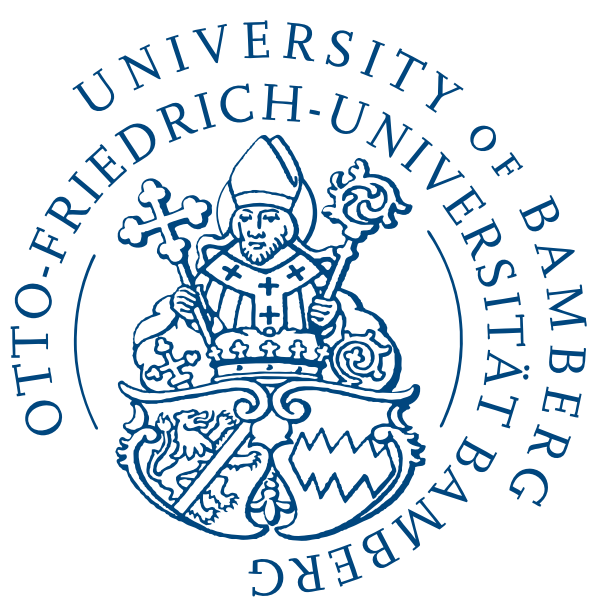

vorgelegt von

Juan Carlos Peña Méndez

März 2021 


\section{Essays on Inequality, Economic Growth and Political Polarization}

\section{INAUGURAL-DISSERTATION}

zur Erlangung des akademischen Grades eines Doktors der Wirtschaftswissenschaften (Dr. rer. pol.)

Fakultät Sozial- und Wirtschaftswissenschaften der Otto-Friedrich-Universität Bamberg

vorgelegt von

Juan Carlos Peña Méndez

Bamberg, März 2021 
Dieses Werk ist als freie Onlineversion über das Forschungsinformationssystem (FIS; https://fis.uni-bamberg.de) der Universität Bamberg erreichbar. Das Werk steht unter der CC-Lizenz CC-BY.

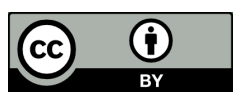

URN: urn:nbn:de:bvb:473-irb-510782

DOI: https://doi.org/10.20378/irb-51078

Datum der Abschlussprüfung: 22. Juni 2021

Erstgutachter:

Prof. Dr. Christian R. Proaño

Professur für Volkswirtschaftslehre, insb. Angewandte Wirtschaftsforschung

Otto-Friedrich-Universität Bamberg

Zweitgutachter:

Prof. Dr. Frank Westerhoff

Lehrstuhl für Volkswirtschaftslehre, insb. Wirtschaftspolitik

Otto-Friedrich-Universität Bamberg

Drittgutachter:

Prof. Mishael Milaković, Ph.D.

Lehrstuhl für Volkswirtschaftslehre, insb. Internationale Wirtschaft

Otto-Friedrich-Universität Bamberg 


\section{Acknowledgements}

This dissertation at hand is the result of my doctorate studies, which I pursued at the graduate program "Bamberg Research Group on Behavioral Macroeconomics (BaGBeM)" as a doctoral candidate. Moreover, I was working at the Professorship for Economics, especially Empirical Economics at the University of Bamberg as a research and teaching assistant and spent a research period at the Macroeconomic Policy Institute (IMK).

This dissertation would not have been possible without the support and cooperation that I received from many people and institutions over the last years. For the different types of aid, I would like to express my sincere gratitude and appreciation. First and foremost, I would like to thank Prof. Dr. Christian R. Proaño, my first thesis supervisor, for the support, guidance and encouragement over the last four years. His supervision and advice helped me to overcome the difficulties I encountered during this thesis. I am very grateful for the space and opportunities he gave me to forge my own path as an independent researcher. I also appreciate his efforts to support my work and other activities such as attending conferences and spending a research period at the IMK that contributed to strengthen my professional profile. Pursuing this dissertation under his supervision has been a remarkable experience.

Second, I would like to thank Prof. Dr. Frank Westerhoff, Chair for Economics, especially Economic Policy, and Prof. Mishael Milaković, Ph.D., Chair for Economics, especially International Economics, both at the University of Bamberg for their openness to my dissertation topic and their willingness to serve as second and third supervisor, respectively. Moreover, I would like to express them my sincerest gratitude for their support, encouragement and organization of multi-day research workshops that made valuable contributions to this dissertation.

I would like to thank the financial support provided by the Hans-Böckler Foundation, which played a key role in achieving this dissertation. Special thanks to Jens Becker and Iris Henkel who were so helpful and provided me assistance throughout specific formal applications. In addition, I would also like to thank the staff of the Hans-Böckler Foundation for having me in events and seminars to acquire additional qualifications.

Moreover, I would like to thank the Economics Department at the University of Bamberg and all its staff for the valuable and satisfying experience. I greatly appreciate the warm and friendly 
atmosphere. Special thanks to Claudia Bauersachs for her constant support with administrative tasks related to the University over the last years.

I would like to thank my co-authors Prof. Dr. Christian R. Proaño, Prof. Dr. Thomas Saalfeld, Jun.-Prof. Dr. Joep Lustenhouwer and Jun.-Prof. Dr. Tomasz Makarewicz. I am delighted to have worked with all of you and I look forward to expand new ideas on future research. I am also very grateful for the professional exchange and the support received from the staff of the IMK during my research period in 2018. Special thanks to Sebastian Gechert, Thomas Theobald, Sebastian Watzka and Sven Schreiber for their thoughtful suggestions for this dissertation. Furthermore, I gratefully acknowledge Prof. Dr. Eric Mayer, head of Research Group Applied Macroeconomics at the University of Würburg, for supporting my arrival at the University of Bamberg.

Last but certainly not least, I would like to thank the following people, without whom this dissertation would not have been possible. My wife, Katharina Schwab, my mother, Julia Méndez, and my uncle, Hugo Méndez, who were so helpful in numerous ways. They had a fundamental role in getting me through the dissertation successfully. This dissertation is dedicated to you. 


\section{Coauthors}

A large part of this dissertation was performed with the productive collaboration of co-authors that I would like to thank for their time, effort and substantial contributions to this thesis.

Chapter 2 is a joint work with Prof. Dr. Christian R. Proaño, Professorship for Economics, especially Empirical Economics, and Prof. Dr. Thomas Saalfeld, Chair of Comparative Politics, both at the University of Bamberg. The title of this project is Inequality, Macroeconomic Performance and Political Polarization: A Panel Analysis of 20 Advanced Democracies. The idea to performance this research project was originated by Christian R. Proaño. Moreover, Thomas Saalfeld contributed with his excellent political knowledge to this research project in many aspects. I contributed with the quantitative research and statistical analysis.

Chapter 3 is a joint work with Prof. Dr. Christian R. Proaño at the University of Bamberg, Jun.Prof. Dr. Joep Lustenhouwer at the University of Heidelberg and Jun.-Prof. Dr. Tomasz Makarewicz at Bielefeld University. The title of this project is Are Some People More Equal than Others? Experimental Evidence on Group Identity and Income Inequality. The idea to performance this research project was originated by Christian R. Proaño and Joep Lustenhouwer. The software for the experimental research was designed by Joep Lustenhouwer. Moreover, Tomasz Makarewicz and I carried out the quantitative analysis of the date obtained from the experiment. Additionally, I contributed by providing my assistance and collaboration to Joep Lustenhouwer and Tomasz Makarewicz in specific tasks in which I gained a lot of computer programming experience. Moreover, we all cooperated to run the sessions in the Bamberg Laboratory for Experimental Research (BLER) at the University of Bamberg throughout the summer term 2019. 


\section{Contents}

$\begin{array}{lll}\text { Acknowledgements } & \text { III }\end{array}$

$\begin{array}{ll}\text { Coauthors } & \text { V }\end{array}$

$\begin{array}{ll}\text { List of Abbreviations } & \text { VII }\end{array}$

$\begin{array}{ll}\text { List of Figures } & \text { IX }\end{array}$

$\begin{array}{ll}\text { List of Tables } & \text { XI }\end{array}$

$\begin{array}{lr}\text { Summary } & 1\end{array}$

1 How is the Growth-Inequality Nexus Influenced by Financial Development? A $\begin{array}{ll}\text { Panel VAR Analysis } & 7\end{array}$

1.1 Introduction . . . . . . . . . . . . . . . . . . . . 8

1.2 Literature Overview . . . . . . . . . . . . . . . . . . . . . 10

1.3 Data and Methodology . . . . . . . . . . . . . . . . . . . 13

1.4 Empirical Results . . . . . . . . . . . . . . . . . . . . . . . . . 19

1.5 Concluding Remarks ............................. . . 31

1.6 Appendix to Chapter 1 . . . . . . . . . . . . . . . . . . . . . 32

2 Inequality, Macroeconomic Performance and Political Polarization: A Panel Anal$\begin{array}{ll}\text { ysis of } 20 \text { Advanced Democracies } & 44\end{array}$

2.1 Introduction . . . . . . . . . . . . . . . . . . . . . . . . . 45

2.2 Literature Overview . . . . . . . . . . . . . . . . . . . . . . . . 47

2.3 Empirical Results . . . . . . . . . . . . . . . . . . . . . 51 


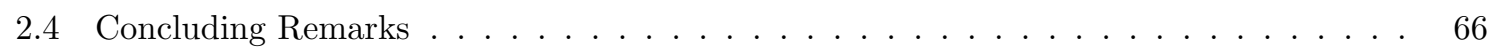

2.5 Appendix to Chapter $2 \ldots \ldots \ldots \ldots \ldots \ldots \ldots$

3 Are Some People More Equal than Others? Experimental Evidence on Group Identity and Income Inequality $\quad 83$

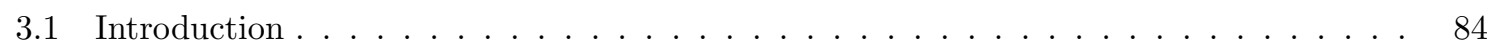

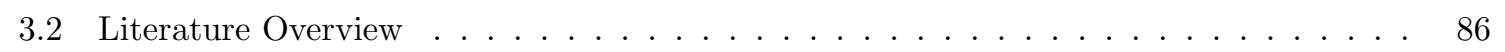

3.3 Experimental Design . . . . . . . . . . . . . . . . . . . . . 90

3.4 Testable Hypotheses and Experimental Procedures . . . . . . . . . . . . . . . . 96

3.5 Experimental Results . . . . . . . . . . . . . . . . . . . . . . . . . 100

3.6 Concluding Remarks . . . . . . . . . . . . . . . . . . . . . . . . . 113

3.7 Appendix to Chapter $3 \ldots \ldots \ldots \ldots \ldots \ldots \ldots \ldots$ 


\section{List of Abbreviations}

\begin{tabular}{ll} 
BLER & Bamberg Laboratory for Experimental Research \\
CE & Colorful Equality treatment \\
CI & Colorful Inequality treatment \\
Ch3040 & Employment chance of either $30 \%$ or $40 \%$ \\
Ch5060 & Employment chance of either $50 \%$ or $60 \%$ \\
Ch7080 & Employment chance of either $70 \%$ or $80 \%$ \\
Ch90 & Employment chance of $90 \%$ \\
EcoGlob & Economic Globalization Index \\
FDI & Financial Development Index \\
FEVD & Forecast error variance decomposition \\
FII & Financial Institutions Index \\
FLVS & Far-left vote share \\
FMI & Financial Markets Index \\
FRVS & Far-right vote share \\
Full_No & Full most preferred choice and No second most preferred choice \\
Full_Partial & Full most preferred choice and Partial second most preferred choice \\
GE & Gray Equality treatment \\
GI & Generalized Method of Moments \\
GMM & Frality treatment \\
\hline
\end{tabular}




\begin{tabular}{|c|c|}
\hline GiniMarket & Market income inequality \\
\hline GiniNet & Net income inequality \\
\hline HFD & High Financial Development \\
\hline IRFs & Impulse response functions \\
\hline LFD & Low Financial Development \\
\hline Low10 & Income share held by the bottom $10 \%$ \\
\hline Low20 & Income share held by the bottom $20 \%$ \\
\hline MVS & Middle vote share \\
\hline No_Full & No most preferred choice and Full second most preferred choice \\
\hline No_Partial & No most preferred choice and Partial second most preferred choice \\
\hline PVAR & Panel vector autoregressive \\
\hline Partial_Full & Partial most preferred choice and Full second most preferred choice \\
\hline Partial_No & Partial most preferred choice and No second most preferred choice \\
\hline PolGlob & Political Globalization Index \\
\hline SWIID & Standardized World Income Inequality Database \\
\hline SocGlob & Social Globalization Index \\
\hline Top10 & Income share held by the top $10 \%$ \\
\hline Top20 & Income share held by the top $20 \%$ \\
\hline
\end{tabular}




\section{List of Figures}

1.1 Cross-country averages over the FDI, FII and FMI . . . . . . . . . . . . . . . . . . 14

1.2 Income inequality measures and financial development . . . . . . . . . . . . . . . . 16

1.3 Cumulative responses to GDP shock using the FDI . . . . . . . . . . . . . . . . . . . 22

1.4 Cumulative responses to GiniMarket shock using the FDI . . . . . . . . . . . . . . . . 24

1.5 Cumulative responses to Low10 shock using the FDI . . . . . . . . . . . . . . . . . . 26

1.6 Cumulative responses to Top10 shock using the FDI . . . . . . . . . . . . . . . . . . . 29

1.7 Forecast error variance decomposition . . . . . . . . . . . . . . . . . . . . 36

1.8 Cumulative responses of all variables using the FMI . . . . . . . . . . . . . . . . . . 38

1.9 Cumulative responses of all variables using the FII . . . . . . . . . . . . . . . . . . . 40

1.10 Cumulative responses of all variables using the GiniNet and the FDI . . . . . . . . . . 42

2.1 Income inequality measures and parliamentary vote shares . . . . . . . . . . . . . . 54

2.2 Time-varying coefficients obtained from GiniNet and SocGlob of FRVS and FLVS . . 60

2.3 Time-varying coefficients obtained from Low10 and Top10 of FRVS and FLVS . . . . . 65

2.4 Time-varying coefficients obtained from EcoGlob and PolGlob of FRVS and FLVS . . 74

3.1 Distribution of employment probabilities . . . . . . . . . . . . . . . . . 91

3.2 Realized voting frequencies for Full, Partial and No insurance schemes . . . . . . . . . 103 


\section{List of Tables}

1.1 Countries at the top and bottom distribution according to the FDI, FII and FMM . . 15

1.2 Information to estimation results . . . . . . . . . . . . . . . . . . . . . 20

1.3 PVAR-Granger causality test (GDP $\Rightarrow$ GiniMarket/Low10/Top10) . . . . . . . . . 23

1.4 PVAR-Granger causality test (GiniMarket $\Rightarrow$ GDP/Low10/Top10) . . . . . . . . . 25

1.5 PVAR-Granger causality test (Low10 $\Rightarrow$ GDP/GiniMarket/Top10) . . . . . . . . . . . 27

1.6 PVAR-Granger causality test (Top10 $\Rightarrow$ GDP/GiniMarket/Low10) . . . . . . . . . . . 30

2.1 Panel OLS regressions with GiniNet (all countries). Sample: 1970-2016 . . . . . . 56

2.2 Panel OLS regressions with GiniNet (all countries). Subsample: 2000-2016 . . . . . . . 58

2.3 Panel OLS regressions with GiniNet (European countries). Sample: 1970-2016 . . . . 60

2.4 Panel OLS regressions with Gini Net (European countries). Subsample: 2000-2016 . . 61

2.5 Panel OLS regressions with 90-10 ratio (all countries). Sample: 1970-2016 . . . . . . 62

2.6 Panel OLS regressions with Low10 (all countries). Sample: 1970-2016 . . . . . . . . . 63

2.7 Panel OLS regressions with Low10 (all countries). Subsample: 2000-2016 . . . . . . . 63

2.8 Panel OLS regressions with Low10 (European countries). Sample: 1970-2016 . . . . . 64

2.9 Panel OLS regressions with Low10 (European countries). Subsample: 2000-2016 . . . 64

2.10 Panel OLS regressions with 90-10 ratio (all countries). Subsample: 2000-2016 . . . . 75

2.11 Panel OLS regressions with 80-20 ratio (all countries). Sample: 1970-2016 . . . . . . 76

2.12 Panel OLS regressions with 80-20 ratio (all countries). Subsample: 2000-2016 . . . . 76

2.13 Panel OLS regressions with Low20 (all countries). Sample: 1970-2016 . . . . . . . . . 77

2.14 Panel OLS regressions with Low20 (all countries). Subsample: 2000-2016 . . . . . 77

2.15 Panel OLS regressions with Low20 (European countries). Sample: 1970-2016 . . . . . 78

2.16 Panel OLS regressions with Low20 (European countries). Subsample: 2000-2016 . . 78 
2.17 Panel OLS regressions with Top10 (all countries). Sample: 1970-2016 . . . . . . . . . 79

2.18 Panel OLS regressions with Top10 (all countries). Subsample: 2000-2016 . . . . . . . 79

2.19 Panel OLS regressions with Top20 (all countries). Sample: 1970-2016 _ . . . . . . . . . 80

2.20 Panel OLS regressions with Top20 (all countries). Subsample: 2000-2016 . . . . . . . . 80

2.21 Panel OLS regressions with Top10 (European countries). Sample: 1970-2016 ... 81

2.22 Panel OLS regressions with Top10 (European countries). Subsample: 2000-2016 . . . 81

2.23 Panel OLS regressions with Top20 (European countries). Sample: 1970-2016 . . . . 82

2.24 Panel OLS regressions with Top20 (European countries). Subsample: 2000-2016 . . . . 82

3.1 Experimental treatments . . . . . . . . . . . . . . . . . . . . . 91

3.2 Features of experimental sessions . . . . . . . . . . . . . . . . . . . 100

3.3 Frequencies of winning strategies and overall votes . . . . . . . . . . . . . . . . 102

3.4 T-test statistics for differences in regimes and votes between each pair of treatments . 102

3.5 Risk neutral payoff maximizing choices for each treatment . . . . . . . . . . . . . . . . 104

3.6 First and second decisions with non-linear employment chances. Base outcome: No_Partial insurance, CE treatment and Ch5060 . . . . . . . . . . . . . . . . 107

3.7 Survey . . . . . . . . . . . . . . . . . . . . . . . . . 112

3.8 Payoffs for Green and Purple participants in the GE treatment . . . . . . . . . . . . . 116

3.9 Payoffs for Green participants in the GI treatment . . . . . . . . . . . . . . . . . . . 117

3.10 Payoffs for Purple participants in the GI treatment . . . . . . . . . . . . . . . . . . . . 117

3.11 Payoffs for Green participants in the CI treatment . . . . . . . . . . . . . . . . . 118

3.12 Payoffs for Purple participants in the CI treatment . . . . . . . . . . . . . . . . . 118

3.13 Payoffs for Green participants in the CE treatment . . . . . . . . . . . . . . . . . 119

3.14 Payoffs for Purple participants in the CE treatment . . . . . . . . . . . . . . . . 119

3.15 Risk Aversion . . . . . . . . . . . . . . . . . . . . . . 136

3.16 First and second decisions with linear employment chances. Base outcome: No_Partial insurance and $\mathrm{CE}$ treatment . . . . . . . . . . . . . . . . . . 138

3.17 First and second decisions with non-linear employment chances. Base outcome: No_Partial insurance, CE treatment and Ch5060 . . . . . . . . . . . . . . . . 138

3.18 First decision with continuous employment chances. Base outcome: No insurance and GI treatment . . . . . . . . . . . . . . . . . . . . 139 
3.19 Employment chance + treatments . . . . . . . . . . . . . . . . . . . . 140

3.20 First and second decisions with linear employment chances. Base outcome: No_Partial insurance and GI treatment . . . . . . . . . . . . . . . . . . . 140

3.21 First and second decisions with non-linear employment chances. Base outcome: No_Partial insurance, GI treatment and Ch5060 . . . . . . . . . . . . . . . . . . 141

3.22 First decision without employment chances. Base outcome: No insurance and CE

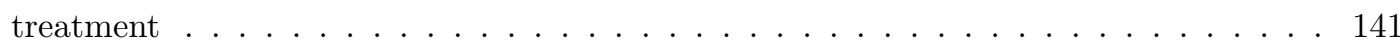

3.23 Frequency probability Ch3040 . . . . . . . . . . . . . . . . . . . . . . 142

3.24 First decision with continuous employment chances. Base outcome: No insurance and $\mathrm{CE}$ treatment . . . . . . . . . . . . . . . . . . . . . . . . 143

3.25 First decision with non-linear employment chances. Base outcome: CE treatment and Ch5060 . . . . . . . . . . . . . . . . . . . . . . . . . 144

3.26 First and second decisions with continuous employment chances. Base outcome: No_Partial insurance and $\mathrm{CE}$ treatment f . . . . . . . . . . . . . . . . 145 


\section{Summary}


Economic inequality has moved into the focus of academic debate in the last decade as it gained increasing public attention in the aftermath of the financial crisis in 2007. According to the estimations of World Inequality Report $2018^{1}$, inequality has increased in most world regions since 1980, but with different magnitudes. In 2016, the top $10 \%$ income share captured the $55 \%, 47 \%, 46 \%$ and $37 \%$ of the total national income in India, United States, Russia and Europe respectively. However, this trend is even more pronounced when looking at individual countries in more detail. One of the most remarkable examples is the United States where, on the one hand, the top 1\% income share increased from $11 \%$ to $20 \%$ from 1980 to 2016 , and, on the other hand, the bottom $50 \%$ declined from $21 \%$ to $13 \%$ in the same period. Some authors argue that inequality is the major social issue of our time since it may unleash different types of economic, political and social problems (Stiglitz 2013 Piketty 2014a, Milanovic, 2016).

From the economics perspective, there is a renewed interest in the literature about the idea that there is a trade-off between income inequality and economic growth. ${ }^{2}$ Theoretical studies provide ambiguous predictions about this relationship. While some theoretical studies suggest that inequality promotes economic growth (Kaldor, 1957, Okun, 2015. Saint-Paul and Verdier, 1993), other studies indicate that inequality can be harmful for economic growth (Perotti, 1996. Galor and Zeira 1993 and Aghion et al., 1999). In addition, concepts such as "trickle-down" and "trickle-up" effects have also recently gained a renewed attention in the academic debate. The former focuses on the accumulation of wealth triggered by economic policies in favor of large companies and high income groups through tax reliefs and facilitation of investment conditions. The increase in wealth of these groups is assumed to be beneficial to all sections of society (Aghion and Bolton, 1997). By contrast, "trickle-up" focuses on the demand side of the economy and it states that poverty constrains demand of low income groups (Van Kesteren et al., 2019). Thus, policies that favor low income groups - generating higher income through access to quality education - will not only alleviate multiple constraints faced by low income groups, but also stimulate economic development by creating additional demand in markets (Thirlwall, 1994).

From a political perspective, the increase of income inequality has been cited as an important contributor to the electoral success of radical and populist parties in the last years. Recent contributions in the economic voting literature - with a special focus on the United States - suggest a close relationship between economic inequality and political polarization (e.g., Duca and Saving 2016 and Voorheis et al., 2015). In Western Europe, traditionally established parties and government coalition parties are challenged by the electoral rise of the National Front in France, the Party for Freedom in the Netherlands, the Alternative for Germany in Germany, the Five Star Movement in Italy, among others. The economic voting literature demonstrates that government parties frequently get punished

\footnotetext{
${ }^{1}$ See Alvaredo et al. (2018).

${ }^{2}$ Moreover, there has been an increasing discussion of "the growth" and "degrowth" paradigms as development policies to achieve economic and social goals in the last years.
} 
(rewarded) for bad (good) performance of the economy. Economic downturns may affect the voters' economic well-being and may lead them to switch from a governing party to a "mainstream" opposition party, or to develop a certain amount of resentment against all mainstream parties and the established political elite. Such general disaffection may be exacerbated in times of economic crisis such as in 2007 and expressed in street demonstrations (Kriesi 2014) or in the support for ideologically extreme parties (de Bromhead et al., 2012, Kriesi, 2014, Bartels, 2014, Funke et al. 2016). In addition, McCarty et al. (2016) stress also the effect of economic inequality on the political landscape and argue that the political polarization in American society may be partially explained by the overall course of fiscal policy and the deregulation of the economy since the Reagan administration.

From a social perspective, perceptions of relative economic positions may affect intergroup behavior and intergroup conflict within a society. The recent immigration waves to European countries and other parts of the world, have intensified social and political tensions about native's perceptions and acceptance of immigrants, their preferences over redistribution and the size of the welfare state. An important example is the Brexit. After the expansion of the EU in 2004, there was a significant increase of immigration of citizens from the new member countries that settled in the UK in search of jobs (Sumption and Vargas-Silva, 2020). This caused a political backlash in the British politics, which, after the 2007 economic crisis and the following recession, culminated with the Brexit referendum that was ultimately won by the "Leave" camp with a narrow margin. A further consequence of this political melee and the narrow victory margin in the referendum was an increased polarization, in terms of political distance, between pro- and anti-EU voters, as well as between Scotland and other British constituent countries. A similar phenomenon can be observed in many Latin American countries with the arrival of Venezuelan migrants in the last years.

Against this background, the motivation for this dissertation arose from the need to gain a deeper understanding about the economic, political and social consequences of income inequality. In particular, this dissertation seeks to address the following questions.

- From the economics perspective:

- Do higher levels of income inequality have a detrimental effect on economic growth?

- Do policies in favor of high income groups have positive effects to the rest of society? Or do investments in low income groups enhance wider economic development?

- From a political perspective:

- Does inequality influence political preferences and thus political polarization?

- Is this political reaction triggered by high or low income groups?

- From a social perspective: 
- Does the relative economic status of the natives influence perceptions and acceptance of immigrants and their willingness for social sharing?

- Does the observed political backlash emerge from an economic anxiety that these immigrants will take over jobs from the native population, and put pressure on the already strained social safety programs? Or is this phenomenon to be interpreted under the aspect of cultural distance between the established population and the arrival of new immigrants?

The present dissertation contributes to the growing and existing literature on income inequality in significant ways. It considers three contributions that empirically analyze the consequences of income inequality. The first essay reviews the link between economic inequality and economic growth using a dataset that covers 110 advanced and developing economies. The second essay investigates the link between political outcomes and income inequality in 20 advanced economies. The third essay examines the relationship between income inequality, group identity and preferences over redistribution.

This dissertation relies on data from several sources also used in related studies. Macroeconomic inequality data were collected from two sources: Standardized World Income Inequality Database SWIID (Solt, 2019) and the World Bank DataBank (World Bank, 2019). The SWIID database maximizes comparability of income inequality across observations and covers a large number of countries and years. Moreover, it provides the distinction between estimates of income inequality pre-tax and post-tax. While the pre-tax Gini coefficient indicates income inequality before taxes and transfers, i.e. market income inequality, the post-tax Gini coefficient indicates income inequality after taxes and transfers, i.e. net income inequality. This dissertation uses the pre-tax and the post-tax Gini coefficients as proxies for average income inequality. Due to the well known limitations of the average income inequality that it underestimates shift at the top and at the bottom of the distribution of income within an economy, other metrics of income inequality are also employed. This thesis uses the income shares held by the top $10 \%$ and $20 \%$, as proxies for high income groups, and the bottom income shares held by the $10 \%$ and $20 \%$, as proxies for low income groups, from the World Bank DataBank. In addition, parliamentary data were obtained from Parliaments and Governments Database (Döring and Manow, 2015), which provides an extensive coverage of general elections in several democratic countries.

From a methodological perspective, this thesis uses a variety of statistical tools to study the implications of income inequality. These include fixed-effects panel regressions, as employed in Mian et al. (2014) and Funke et al. (2016), to wipe out country-specific time constant unobserved heterogeneity. Next, panel vector autoregressive models, as employed in Atems and Jones (2015) and Jeong and Kim (2018), to analyze the feedback interaction of the endogenous variables within a system of linear equations. To disentangle the impact of exogenous shocks, this thesis relies on a standard Choleski decomposition (Sims, 1980) which imposes recursive zero restrictions on contemporaneous structural parameters to the reduced form representation. Moreover, this dissertation includes a laboratory ex- 
periment that allows to elicit opinions and preferences for different political schemes in a controlled environment. The experiment was programmed using oTree (Chen et al. 2016) and it was conducted in German language (including instructions, graphical user interface and oversight) during the summer term 2019. To test the statistical significance of the results obtained from the experiment, this essay employs a multinomial logistic regression model with individual random effects and different latent dependent variables. Moreover, all essays provide a wide range of robustness checks of the main estimates.

Chapter 1 explores the dynamic relationship between economic growth and the different metrics of income inequality using a compiled dataset covering 110 advanced and developing economies from 1980 to 2016. In addition, based on the notion that the country's financial development level itself may play an important role in the Growth-Inequality nexus (Greenwood and Jovanovic, 1990), this essay uses as a sample splitting criterion the Financial Development Index (FDI), provided by Sahay et al. (2015), which takes into account depth, access, and efficiency of financial markets and institutions. The empirical exploration shows that more economic growth leads in general to a more equal pre-tax average income distribution. In addition, a country's financial development level plays an important role in the relationship between economic growth and income inequality (Levine 2005). Countries with high levels of financial development are more efficient in reducing pre-tax average inequality and inequality for low income groups. Moreover, a high pre-tax average income inequality is associated with lower economic growth, although this effect seems to be less strong for countries with low levels of financial development. This finding supports previous research provided by Alesina and Rodrik (1994), Dabla-Norris et al. (2015) and Berg et al. (2018). Additionally, it seems that there is a bi-directional relationship between economic growth and pre-tax average income inequality for countries with a high financial development level. Furthermore, the results highlight the importance of a strong middle income class (pre-tax average income inequality) for a more equitable income distribution within an economy (Kharas, 2010). Finally, it seems that there is a possible "trickle-down" mechanism and a mutual interdependence of pre-tax average and low income groups for countries with low levels of financial development.

Chapter 2 examines the macroeconomic and social determinants of voting behavior, and especially of political polarization, using a compiled dataset for 20 advanced economies from 1980 to 2016 which covers 291 parliamentary elections. This essay defines political polarization as the electoral support for such parties of the extreme left and right. The identification of parties according to their ideological position was based on the party codification by Funke et al. (2016). Far-left (far-right) vote share is calculated by summing up all parliamentary seats of those parties ranging from left (right)-wing populism to the radical left (right) in a given election period. Moreover, this essay controls for various macroeconomic variables used in related studies such as real GDP per capita, unemployment rate, globalization among others. The key finding is that high levels of income inequality can influence electoral choices. More specifically, both post-tax average inequality and low income groups are 
statistically linked to the recent success of far-right parties, while high income groups not. The link of income inequality and political polarization thus seems to be based on the deterioration of the relative economic position especially of the poorest fraction of the population. In addition, the analysis indicates that a significant change in the link between income inequality and political polarization appears to have taken place over the last twenty years.

Chapter 3 investigates the effects of group identity and income inequality on social preferences and polarization by means of a laboratory experiment. In other words, this essay examines whether political polarization and an unwillingness for redistribution are mainly driven by factors associated to relative economic status (income inequality), or rather by social identity and group formation. The experiment splits the subjects into two populations: in-group (representing "natives") and out-group ("migrants"). In-group subjects repeatedly vote whether an unemployment insurance should cover all, some, or no members of their group. By means of a two-by-two design the experiment disentangles the effect of group identity from those of income inequality. In general, the experiment suggests a complex relationship between voting, income distribution and group identity, and by extension, migration. The main results can be summarized as follows. First, subjects in all treatments tend to vote for less inclusive insurance schemes when they sample a higher chance of employment. At the same time, subjects with low employment chances were more likely to cover some subjects if the insurance scheme implies being covered with higher benefits at a lower taxation level. Second, in-group subjects with an ex ante more beneficial distribution of employment chances - relative to the out-group - are less selfish and vote for more inclusive insurance schemes. Third, ex ante more beneficial relative employment chances of in-group subjects also lead to less polarization, i.e. less voting heterogeneity. Finally, revelation and priming of group identity - contrary to popular political discourse - does not lead to discrimination against out-group "migrants" but, on the contrary, can lead to more compassionate and inclusive attitudes. 


\section{Chapter 1}

How is the Growth-Inequality Nexus Influenced by Financial Development? A Panel VAR Analysis ${ }^{1}$

${ }^{1}$ I would like to thank Christian R. Proaño for helpful comments and suggestions on previous drafts of this essay. 


\section{$1.1 \quad$ Introduction}

Two of the most outstanding developments of the last thirty years have been the remarkable liberalization of financial markets both within countries as well as across borders (Stiglitz. 2000) and the dramatic increase in income and wealth inequality (Atkinson et al. 2011, Stiglitz 2013, Piketty 2014b). Against this background, it is today more than ever of central importance to gain a deeper understanding of the interaction between financial development, income distribution or inequality and economic growth.

The Finance-Growth nexus has been widely studied in the economics literature over the years since the classical works by Schumpeter (1912), Goldsmith (1969), McKinnon (1973), Shaw (1973) and others, both theoretically and empirically. As discussed by Cochrane (2009), the benefits of an enlargement of the existing financial system lie primarily in a better diversification and management of risk, as an expansion of the set of traded financial assets is likely to cover a larger set of possible aggregate outcomes, or 'states of nature' (Arrow and Debreu 1954). The notion that a well-functioning financial system can contribute to economic growth by allowing for a more efficient allocation of resources is widely supported by empirical evidence (see e.g. King and Levine 1993 and Beck and Levine, 2004). However, the contribution of financial development on economic growth seems to be of a nonlinear nature. For example, Rioja and Valev (2004) find that the impact on growth of a marginal increase in financial development is small in countries with a low level of financial development, large in rich countries and particularly large in middle-income countries. By contrast, the theoretical and empirical literature on the Finance-Inequality nexus provides only an ambiguous picture of the distributional effects of finance and economic growth, as it is still unclear which segment of the society benefits mostly from financial development. Similarly, the Growth-Inequality nexus seems ambiguous and, as pointed out by Greenwood and Jovanovic (1990), possibly dependent on the stage of economic development. There seems thus to exist an inextricable and multidirectional link between economic growth, financial development and income inequality.

This paper seeks to shed more light into the Growth-Inequality nexus by providing a more holistic description of the dynamic relationship of economic growth and income inequality at different stages of financial development using panel vector-autoregressions (PVARs). More specifically, this paper employs different measures of income inequality such as average and tails income inequality to have a more clear characterization of the income inequality within an economy. We use the pre-tax Gini coefficient, as proxy for average inequality, and the income shares held by the bottom and top 10\%, as proxies for low and high income groups respectively. In other words, we investigate how higher economic growth is distributed across different (high, average and low) income groups within a society by different levels of financial development and; how economic growth and our measures of income inequality are affected by a change of a particular income class. As discussed later in more details, we 
use the Financial Development Index (FDI) provided by Sahay et al. (2015) which takes into account depth, access, and efficiency of financial institutions and markets, as proxy for financial development. Although financial development may impact economic growth and income inequality directly and indirectly, this paper mainly focuses on the latter. This means that we explore the feedback dynamics of economic growth and (average and tails) income inequality for given levels of financial development. To the best of our knowledge, this is one of the first studies that examines the dynamics of the GrowthInequality nexus taking into account different levels of financial development using a large sample data, something rarely done in the literature.

Our main results can be summarized as follows. First, we find that more economic growth leads in general to a more equal average income distribution. Furthermore, it seems that countries with high financial development levels are more efficient in reducing inequality for low income groups. Second, a high average income inequality is associated with lower economic growth, although this effect seems to be less strong for countries with low levels of financial development. This finding supports previous research provided by Alesina and Rodrik (1994), Persson and Tabellini (1994), Dabla-Norris et al. (2015) and Berg et al. (2018). Third, our results highlight the importance of a strong middle income class for a more equitable income distribution within an economy (Kharas 2010). We find that higher average inequality leads to a more unequal income distribution since low income groups become poorer and high income groups richer irrespective of the country's financial development level. Fourth, a bi-directional association may exit between average inequality and low income groups for countries with low levels of financial development, which implies that these two groups of society are more vulnerable to external shocks. By contrast, this is not the case for countries with high levels of financial development where it seems that a deterioration of average and/or low income groups will be, at least partially, compensated by the access to financial markets and the relaxation of credit constraints. These results are in line with Levine (2005) who stresses the positive effect of a well-functioning financial system on society. Finally, our results suggest the existence of 'trickle-down effect' of capital accumulation (Aghion and Bolton, 1997) in countries with low levels of financial development, in which an increase of high income groups reduces inequality by improving both average and low income groups. In addition, economic growth reacts positively to an increase of high income groups, but this response is statistically insignificant.

From a methodological point of view, three papers are closest to ours. Atems and Jones (2015) investigate the Growth-Inequality nexus for a panel of states in the United States from 1930 to 2005 and conclude that higher Gini coefficient decrease economic growth. However, they cannot find statistical evidence that economic growth affects the Gini coefficient. Similarly, Jeong and Kim (2018) find that the pre-tax Gini coefficient has a significant negative effect on economic growth covering 174 countries for the 1960-2013 period using PVARs. In contrast, da Silva (2020) shows that inequality has a positive effect on economic growth using state-level data for Brazil from 1992 to 2011. Moreover, the author also finds that a positive GDP shock reduces inequality. Although these results provide 
some insights into the dynamic between income inequality and economic growth, it is still unclear how more economic growth is distributed across society since previous studies have not treated (average and tails) income inequality in much detail.

The remainder of this paper is organized as follows. A review of the existing literature is presented in Section 1.2. While Section 1.3 presents the data description and methodology, Section 1.4 discusses the estimations results of the PVAR. Finally, Section 1.5 concludes this paper.

\subsection{Literature Overview}

The Growth-Inequality nexus was initially investigated by Kuznets (1955), who pointed out that the relationship between income inequality and income per capita can be described by an inverted U-curve, in which income inequality increases and decreases in the transformation process from a traditional agricultural economy to a modern industrial economy. ${ }^{2}$ After a period where this nexus seemed to be of second-order importance in academic circles, it has recently gained more attention again due to the growing income and wealth inequality in the last decades (Atkinson et al. 2011. Stiglitz 2013 Piketty, 2014b).

Theoretical studies provide ambiguous predictions about the Growth-Inequality nexus. ${ }^{3}$ Kaldor (1957) argues that a more unequal distribution leads to a higher aggregate savings rate and a higher economic growth: As wealth and income inequality influence the average marginal propensity to save, a more unequal income distribution fosters saving and investment since high income groups have a higher propensity to save, enhancing the process of development via capital accumulation. Similarly, Okun (2015), when discussing the potential "big trade-off" between equality and efficiency, argues that redistributive policies or less economic inequality may not only reduce the incentive to work, but also economic activity in general. He concludes that inequality increases innovative processes and thus economic growth. Further, Saint-Paul and Verdier (1993) provide a theoretical framework to describe that democracies with high levels of inequality elect higher taxation schemes to finance education spending which promotes economic growth via capital accumulation.

Other theoretical studies indicate that inequality can be harmful for economic growth. Perotti (1996), Galor and Zeira (1993) and Aghion et al. (1999) argue that inequality can be harmful for

\footnotetext{
${ }^{2}$ At early stages of economy development, he states that average income was lower in rural areas than in cities; and income inequality increases through urbanization processes. As economy develops, the new generations coming from rural areas enjoy the new possibilities provided by the cities. They take jobs with higher wages and income inequality decreases.

${ }^{3}$ In recent years, there has been an increasing discussion of "the growth" and "degrowth" paradigms as development policies to achieve economic and social goals. While the former focus on economic growth as a policy goal, the latter focus on environmental policies to achieve a reduction of our consumption of emissions-intensive goods and thus achieve high levels of human well-being. For a full discussion about theoretical and empirical studies about these two paradigms see Kallis et al. (2018) and Buch-Hansen and Koch (2019).
} 
economic growth due to borrowing constraints and investment in education issues. The existence of fixed costs for investment in education may discourage low income groups to invest little or nothing in education since they are much more affected due to lack collateral and credit history. Persson and Tabellini (1994) and Alesina and Rodrik (1994) state that high levels of inequality increase pressure for redistribution through a higher rate of taxation that decreases economic growth, see also Meltzer and Richard (1981), De La Croix and Doepke (2003), Ostry et al. (2014) and Berg et al. (2018).

Economic and political debates about the Growth-Inequality nexus continued over the 1980s and 1990s bringing new concepts such as "trickle-down" and "trickle-up" effects. The former focuses on the accumulation of wealth triggered by economic policies in favor of large companies and high income groups through tax reliefs and facilitation of investment conditions. The increase in wealth of these groups is assumed to be beneficial to all sections of society (Aghion and Bolton, 1997). By contrast, "trickle-up" focuses on the demand side of the economy and it states that poverty constrains demand of low income groups (Van Kesteren et al., 2019). Thus, policies that favor low income groups - generating higher income through access to quality education - will not only alleviate multiple constraints faced by low income groups, but also stimulate economic development by creating additional demand in markets (Thirlwall, 1994).

Empirical research was limited until mid-1990 due to the insufficient data for income inequality. However, the increasing availability of data on a large number of countries allowed to exploit crosscountry studies in the last decades. Benabou (1996) provides an overview of empirical studies of the Growth-Inequality nexus using cross-country OLS estimations in which they deliver the same message, namely that income inequality is detrimental to economic growth. Using a variety of cross-country data sets and periods, Alesina and Rodrik (1994), Persson and Tabellini (1994) and Perotti (1996) show that more unequal societies have a detrimental effect on economic growth. However, Li and Zou (1998) and Forbes (2000) overturned these findings showing that there is a positive impact of inequality on economic growth using five-year averaged data and controlling country-specific fixed effects.

De Dominicis et al. (2006) suggest that the Growth-Inequality nexus should be restricted to a more homogeneous countries that share similar socio-economical characteristics. Barro (2000) argues that the impact of inequality on growth may differ between poor and rich countries. Splitting approximately 100 countries into rich and poor countries, the author finds that the relationship between inequality and growth rates is non-linear and while higher inequality is associated with lower growth in poor countries, higher inequality is associated with higher growth in rich countries.

A more recent strand of the empirical literature employs the System Generalized Method of Moments (GMM) estimator proposed by Arellano and Bover (1995) to address endogeneity issues. For example, Ostry et al. (2014), Dabla-Norris et al. (2015) and Berg et al. (2018), using System GMM estimations, find a negative association between income inequality and economic growth averaging 
data over fixed length intervals. More related to the methodology used in this paper and discussed in more detail in Section 1.3.2, studies like Atems and Jones (2015) examine the causality effect between economic growth and income inequality using PVAR models for states of the US from 1930 to $2005 .^{4}$ The author show that causality effects goes from inequality to economic growth but not the other way around. Similarly, Jeong and Kim (2018) find that average income inequality decreases significantly economic growth using 174 countries from 1960 to 2013 period. By contrast, da Silva (2020) shows that inequality has a positive effect of economic growth using state-level data for Brazil from 1992 to 2011, while a positive GDP shock reduces inequality.

An additional strand on the literature has focused on the role of financial development for economic growth and inequality. According to Levine (2005), a well-developed financial system may promote economic growth by the reduction of market frictions, i.e. information and transaction costs, since financial intermediaries allocate efficiently savings to high return projects, generate more investment opportunities, and offer more risk diversification services associated with individual projects. ${ }^{5}$

Increasing the access and availability of financial services to those participants who previously were not part of the financial system due to financial restrictions may however also affect a society's income inequality. Theoretical studies such as Galor and Zeira (1993), Banerjee and Newman (1993) and Galor and Moav (2004) predict that deeper finance reduces income inequality. These authors argue that financial intermediaries will allocate capital and resources more efficiently when financial market and institutions work well. The reduction of financial frictions benefits the society as a whole, specially low income groups, via investment in human capital, and financially constrained firms, via access to external finance. On the other hand, Greenwood and Jovanovic (1990) argue the existence of a nonlinear association between finance and inequality by which the effects of finance on income distribution depends on the level of economic development: At initial stages of development income inequality may increase since only high income groups have the possibility to enjoy financial services. As development continues, income inequality is likely to decrease as the poorer rest of the population gain access to the financial system and benefit from its financial services. ${ }^{6}$

A common feature of the aforementioned set of empirical studies on the Growth-Inequality nexus is that they use the Gini coefficient as the measure of income inequality. However, it is well known that this measure underestimates changes at the tails of the income distribution (Atkinson 1970). By

\footnotetext{
${ }^{4}$ As it will be discussed below, the PVAR methodology combines cross-sectional as well as time series variation and captures the interactive feedback dynamics among the variables, i.e. the effects in both directions.

${ }^{5}$ Levine (2005) argues that a financial system has five major functions through which it can influence on economic growth, namely a) the production of information ex ante about possible investments and the allocation of capital, b) the monitoring of investments and the exertion of corporate governance after providing finance, c) the diversification and management of risk, d) the collection and aggregation of savings and e) the facilitation of exchange of goods and services.

${ }^{6}$ The empirical evidence on the impact of financial development on income inequality and how this effect is distributed to all sections of society is inconclusive and, given the focus of the present paper on the Growth-Inequality nexus, lies beyond its scope.
} 
contrast, this paper aims to contribute the existing literature by analyzing the dynamic relationship between economic growth and (average and tails) income inequality taking into account different stages of financial development using PVARs. ${ }^{7}$ The next section provides detail information about the variables used in this paper.

\subsection{Data and Methodology}

\subsubsection{Data description}

For our analysis we use an unbalanced panel of 110 countries of an annual frequency ranging from 1980 to $2016{ }^{8}$ Our indicators for average income inequality, the pre-tax and the post-tax Gini coefficients (denoted GiniMarket and GiniNet, respectively), stem from the Standardized World Income Inequality Database (SWIID), Versions 8-9 (Solt, 2019). We employ the SWIID database for several reasons. First, the SWIID database covers a larger numbers of countries and years compared to other inequality datasets, such as the Luxembourg Income Study, see Solt (2009). Second, the SWIID database maximizes comparability of income inequality across observations giving the opportunity to realize a more coherent cross-national study. For instance, Acemoglu et al. (2015) uses the SWIID to examine the impact of democracy on inequality on a large number of countries. Third, the SWIID provides estimates of income inequality pre-tax and post-transfer. While GiniMarket indicates income inequality before taxes and transfers, i.e. market income inequality, GiniNet indicates income inequality after taxes and transfers, i.e. net income inequality. Both coefficients range from 0 to 100 , where 0 means perfect equality and 100 means perfect inequality. The difference between these two measures of income inequality is obviously due to the cross-country policy government intervention. We follow Berg et al. (2018) and Jeong and Kim (2018) and use the market income inequality (GiniMarket), abstracting to some extent from the redistributional effect of fiscal policy. Nonetheless, we also use the net income inequality (GiniNet) coefficient to test the robustness of our results.

As previously mentioned, a main shortcoming of the Gini coefficient (either pre- or after tax) is the well known fact that it underestimates changes at the tails of the income distribution (Atkinson 1970). Since we intend to measure the impact of economic growth on tails of the income distribution at different stages of financial development, we use the income shares of the bottom 10\% (Low10) and of the top 10\% (Top10) from the World Bank (2019) as additional endogenous variables. The use of these income shares is motivated by the fact that average income inequality provides only a very limited account of the factual distribution of income within an economy, as well as because the

\footnotetext{
${ }^{7}$ In the following, we will not investigate how economic growth and income inequality may affect financial development, but leave this dimension for further research.

${ }^{8}$ Appendix 1.6 contains the list of countries, the definitions and summary statistics of all variables used throughout this paper.
} 
sharp increase in overall income inequality over the last decades has been primarily driven by an overproportional rise of income at the top of the distribution (Piketty, 2014b).

Following De Dominicis et al. (2006) and based on the notion that financial development itself may play an important role in the Growth-Inequality nexus (Greenwood and Jovanovic 1990), we use as a sample splitting criterion the Financial Development Index (FDI), provided by Sahay et al. (2015), which ranges from 0 (lowest value of financial development) to 1 (highest value of financial development). The FDI index takes into account depth (size and magnitude of liquidity of markets), access (ability to access financial services), and efficiency (ability of institutions to provide financial services at low cost and with sustainable revenues, and the level of activity of capital markets), and it can itself be decomposed into the Financial Institutions Index (FII) and the Financial Markets Index (FMI). While the FII covers banks, mutual funds, insurance firms, pension funds and other types of nonbank financial institutions, the FMI covers mainly bond and stock markets. ${ }^{9}$

(a) Average distribution for FDI

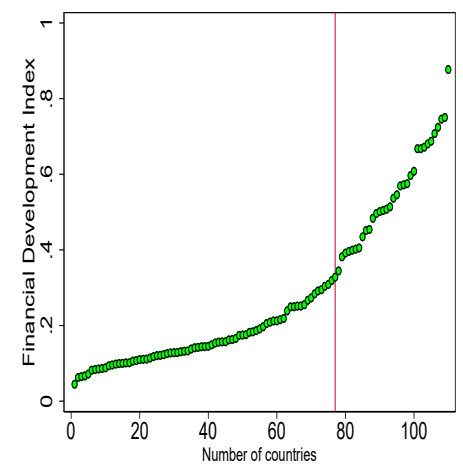

(b) Average distribution for FII

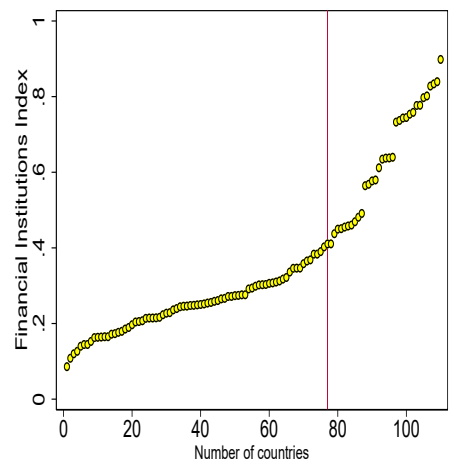

(c) Average distribution for FMI

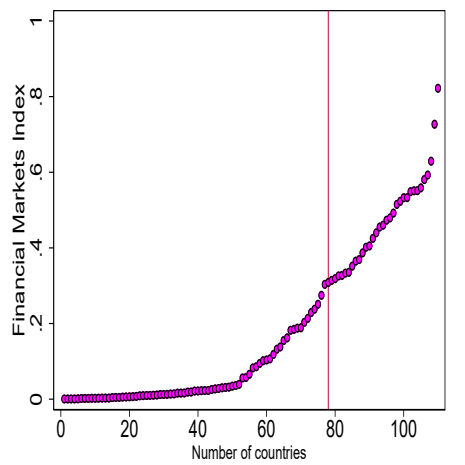

Figure 1.1: Average distributions of Financial Development Index (FDI), Financial Institutions Index (FII) and Financial Markets Index (FMI).

Figure 1.1 shows the cross-country averages over the whole sample period of the FDI (1.1a), FII (1.1b) and FMI (1.1c), respectively. The graph illustrates the large differences across countries in our sample since most of them are located in low ranges of the overall distribution for all three indexes. These differences are the largest for the FMI. From this point onwards (including our estimation results presented in Section 1.4), we use the FDI (Figure 1.1a) since it summarizes the FII and the FMI. Nonetheless, we use the FII (Figure 1.1b) and the FMI (Figure 1.1c) independently to test the sensitivity of our results. We split the country sample into two subgroups: Countries above the $70^{\text {th }}$ percentile (red line) of overall average distributions are classified as High Financial Development (HFD) countries, and countries below the $70^{\text {th }}$ percentile are classified as Low Financial Development

\footnotetext{
${ }^{9}$ For a full description of all categories of every single index, see Svirydzenka (2016).
} 
(LFD) countries. ${ }^{10}$ Accordingly, we have 110 (ALL) countries in which 77 are classified as High Financial Development (HFD) countries and 33 as Low Financial Development (LFD) countries. ${ }^{11}$

Table 1.1 reports the top 10 and bottom 10 countries according to FDI, FII and FMM respectively, showing there is little cross-country variation considering the extremes values of the average distributions.

Table 1.1: Countries at the Top and Bottom Distribution according to alternative Financial Development Metrics

Financial Development Index

\begin{tabular}{ll|ll}
\multicolumn{3}{c|}{ Bottom 10 } & \multicolumn{2}{c}{ Top 10} \\
110. Tajikistan & 105.Mozambique & 1. Switzerland & 6. Canada \\
109. Sierra Leone & 104.Sudan & 2. United States & 7. Netherlands \\
108. Turkmenistan & 103.Cameroon & 3. United Kingdom & 8. Germany \\
107. Guinea & 102.Madagascar & 4. Australia & 9. Ireland \\
106. Uganda & 101.Angola & 5. Japan & 10. Spain \\
\hline
\end{tabular}

Financial Institutions Index

Bottom 10 Top 10

\begin{tabular}{ll|ll} 
110. Tajikistan & 105.Angola & 1. Switzerland & 6. Canada \\
109. Turkmenistan & 104.Mozambique & 2. Japan & 7. Ireland \\
108. Sierra Leone & 103.Chad & 3. United Kingdom & 8. Portugal \\
107. Guinea & 102.Paraguay & 4. Australia & 9. Spain \\
106. Uganda & 101.Azerbaijan & 5. Netherlands & 10. Denmark \\
\hline
\end{tabular}

\begin{tabular}{ll|ll}
\hline \multicolumn{4}{c}{ Financial Markets Index } \\
\cline { 3 - 4 } & & \multicolumn{2}{c}{ Top 10} \\
110. Myanmar & 105.Cameroon & 1. Switzerland & 6. Sweden \\
109. Uganda & 104.Tajikistan & 2. United States & 7. Spain \\
108. Sudan & 103.Nicaragua & 3. United Kingdom & 8. Canada \\
107. Bosnia and Herzegovina & 102.Moldova & 4. Australia & 9. Japan \\
106. Nepal & 101.Lesotho & 5. Germany & 10. Ireland \\
\hline \hline
\end{tabular}

\footnotetext{
${ }^{10} \mathrm{~A}$ similar procedure was also applied for FII and the FMI in the robustness estimations.

${ }^{11}$ Similar results as the ones discussed below were obtained with the $65^{\text {th }}$ and $75^{\text {th }}$ percentiles as threshold values.
} 
Figure 1.2 illustrates the average evolution of Gini coefficients and tail income inequality measures over the last decades. The first row of Figure 1.2 shows the development, on average, of the GiniMarket (Figure 1.2a), the GiniNet (Figure 1.2b) and the difference between the former and the latter (Figure 1.2c) for ALL (solid line), HFD (dashed line) and LFD (dotted line) countries. Interestingly, it can be seen from Figure 1.2a that the GiniMarket in HFD countries started to increase gradually during the mid-1980s, which coincides with the wave of financial liberalization in advanced economies, and exceeds inequality existing in ALL and LFD countries since the beginning of the 1990s. However, HFD countries have been more successful in reducing the GiniNet as shown in Figure 1.2b. While the average evolution of the GiniNet in HFD countries is by $33 \%$ over the last decades, the average evolution of the GiniNet in ALL and LFD countries is by $37 \%$ and $41 \%$, respectively. It is also worth noting that there is a slight increase of the GiniNet in the last years of the sample in all three categories.

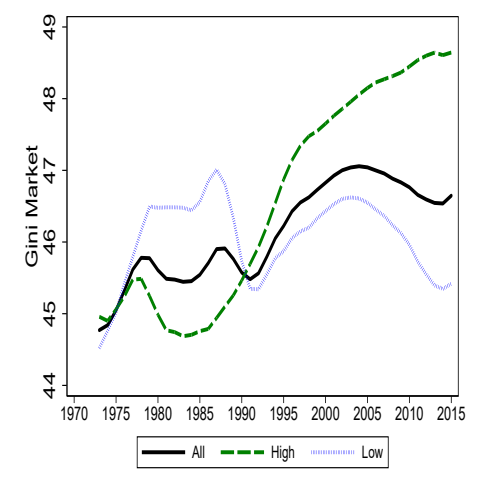

(a) Gini Market

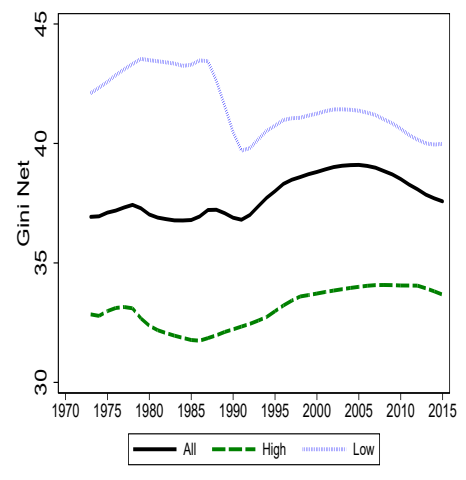

(b) Gini Net

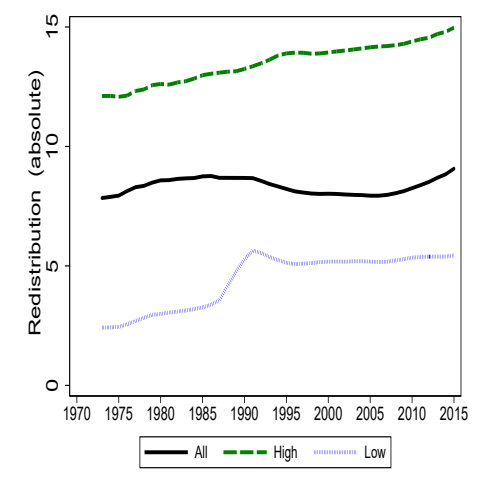

(c) Redistribution

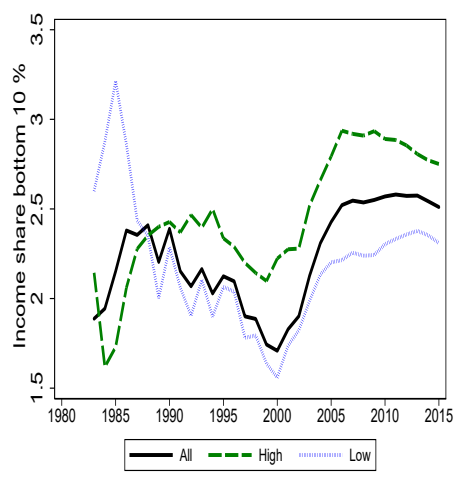

(d) Income share bottom $10 \%$

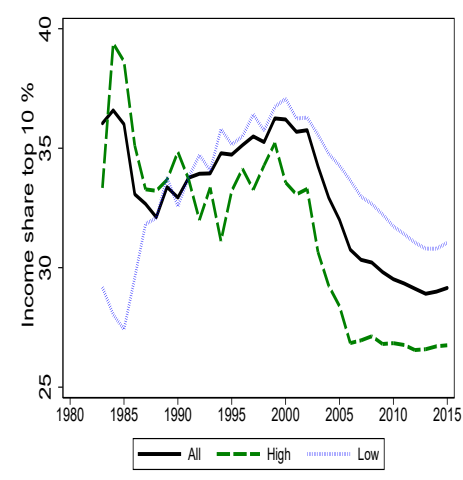

(e) Income share top $10 \%$

Figure 1.2: Average Gini coefficients and tails income inequality measures for ALL countries (solid line), High development (dashed line) and Low Financial Development countries (dotted line). Data are presented using four-year moving average for all variables.

Figure 1.2c illustrates the structural differences between HFD and LFD countries and the extent 
to which government interventions have played a key role to reduce net inequality in HFD countries. ${ }^{12}$

The second row of Figure 1.2 shows the average evolution of the income share held by the bottom $10 \%$ (Low10) and the top 10\% (Top10) for ALL (solid line), HFD (dashed line) and LFD (dotted line) countries as illustrated in Figure 1.2d and Figure 1.2e, respectively. On the one hand, Figure 1.2d shows a gradual increase of the Low10 in HFD countries since the mid-1980s from $1.6 \%$ in 1985 to $2.5 \%$ in 1995 . Then, the Low10 declines until 1999 and then rises steadily over the following years reaching $3 \%$ in 2005, although a slight decrease in the last years can be observed. On the other hand, Figure $1.2 \mathrm{~d}$ shows a steady decline of the Low10 from $3.2 \%$ in 1985 to $1.5 \%$ in 2000 in LFD countries, after which the Low10 increases gradually up to $2.3 \%$ in 2015. A similar picture can be observed in Figure 1.2e where HFD countries has been reducing the income shares of high income groups more rapidly than LFD countries over the last decades. The Top10 decreases considerably from $35 \%$ in 1985 to $25 \%$ in 2015. By contrast, the Top10 in LFD countries increases the first years of the sample from $27 \%$ in 1985 to $36 \%$ in 2000 , after which the Top10 declines continuously to reach $31 \%$ until 2015 .

\subsubsection{Methodology}

To examine more formally the dynamic interaction between economic growth and (average and tails) income inequality, we estimate a panel VAR introduced by Holtz-Eakin et al. (1988) using annual data. In general terms, our model is represented by a system of linear equations where the reduced-form can be expressed as

$$
\mathbf{Y}_{i t}=\mathbf{Y}_{i t-1} \mathbf{A}_{1}+\mathbf{Y}_{i t-2} \mathbf{A}_{2}+\ldots+\mathbf{Y}_{i t-p} \mathbf{A}_{p}+\mu_{i}+\epsilon_{i t}
$$

where $i \in\{1,2, \ldots, N\}$ and $t \in\{1,2, \ldots, T\}$ denote the respective country and time indices, $p$ the number of lags, $Y_{i t}$ a $1 \times k$ vector of endogenous variables, $Y_{i t-j}$ a $1 \times k$ vector of lagged endogenous variables, $A_{j}$ a $k \times k$ matrix that captures the coefficients for endogenous variables, and $u_{i}$ and $\epsilon_{i t}$ are $1 \times k$ vectors of (country- and) variable-specific panel-fixed effects and idiosyncratic shocks, respectively. Following Abrigo and Love (2016), we assume $E\left[\epsilon_{i t}\right], E\left[\epsilon_{i t}^{\prime} \epsilon_{i t}\right]=\Sigma$ and $E\left[\epsilon_{i t}^{\prime} \epsilon_{i s}\right]=0$ for all $t>s$. Put it differently, we assume that country-specific disturbances may be only contemporaneously correlated with each other, both not over time neither with themselves nor with any other. The vector of endogenous variables consists of the growth rate of real GDP per capita (GDP), pre-tax income inequality (GiniMarket), the bottom 10\% income share (Low10) and the top $10 \%$ income share (Top10), i.e. $\mathbf{Y}_{i t}=[$ GDP, GiniMarket, Low10, Top10].

We estimate Equation (1.1) using GMM, which allows for unobserved cross-country heterogeneity as country fixed effects, using use the Stata package developed by Abrigo and Love (2016). The key advantage of the GMM estimator is that potentially endogenous right-hand side variables are

\footnotetext{
${ }^{12}$ The absolute redistribution is the difference between the Gini Market and the Gini Net.
} 
instrumented using appropriate lags of the endogenous variables and additional moment conditions as "internal" instruments to control for endogeneity (Arellano and Bond, 1991 Roodman 2009; Berg et al., 2018). We use the forward orthogonal transformation (FOD) (Arellano and Bover 1995) to remove the country fixed effects which minimizes missing observations. ${ }^{13}$ To verify the validity of our instruments, we report the results of the standard Hansen test of over-identifying restrictions under the null hypothesis that the instrumental variables are not correlated with the residual. A no rejection of the null hypothesis supports the joint validity of the selected instrument set. We set the lag length equal to one based on the Bayesian information criteria (BIC) and Akaike information criteria (AIC) as shown in Appendix 1.6.

For identification, we rely on a standard Choleski decomposition (Sims, 1980). This identification imposes recursive zero restrictions on contemporaneous structural parameters to the reduced form, $u_{i t}=A_{0}^{-1} \epsilon_{i t}$. Here $A_{0}^{-1}$ is used to orthogonalize the residuals as

$$
u_{i t}=\left[\begin{array}{cccc}
a_{11}^{0} & 0 & 0 & 0 \\
a_{21}^{0} & a_{22}^{0} & 0 & 0 \\
a_{31}^{0} & a_{32}^{0} & a_{33}^{0} & 0 \\
a_{41}^{0} & a_{42}^{0} & a_{43}^{0} & a_{44}^{0}
\end{array}\right]^{-1}\left(\begin{array}{c}
\epsilon_{i t}^{G D P} \\
\epsilon_{i t}^{G i n i M} \\
\epsilon_{i t}^{\text {Low } 10} \\
\epsilon_{i t}^{T o p 10}
\end{array}\right)
$$

The first variable introduced in the model is the 'most exogenous' and the last variable is the 'most endogenous'. This implies that the first variable in the model does not contemporaneously respond to any other variable and the last variable responds contemporaneously to all other variables in the model. We order the variables as follows: GDP, GiniMarket, Low10 and Top10 as presented in Equation (1.2). Following Atems and Jones (2015) and Jeong and Kim (2018), we assume that our measures of (average and tails) income inequality are contemporaneously endogenous to economic growth. Moreover, as pointed out by Kharas (2010, p.7) "Underpinning the performance of the G7, and indeed driving the global economy, is a large middle class", we assume that the middle class performs a crucial function for entrepreneurship and innovation that contributes to reduce income inequality within an society. For this reason, we assume that the Low10 and the Top10 are contemporaneously endogenous to GiniMarket. Finally, we assume that the Top10 is the most endogenous variable since it is likely based on multiple income sources as wages and capital gains (Owyang and Shell, 2016). ${ }^{14}$

An advantage of using the PVAR methodology is that it captures the effects in both directions. This implies that we can explore not only how higher economic growth is distributed across high, average, low income groups, but also how economic growth and our measures of income inequality are affected by a change of a particular income class. In other words, we can also investigate the impact of the Low10 and the Top10, which may be related to "trickle-up" and "trickle-down" effects respectively, on the endogenous variables.

\footnotetext{
${ }^{13}$ For a detail discussion of the panel VAR approach, see Abrigo and Love (2016).

${ }^{14}$ To test the sensitivity of our results, we also change the identitication assumption on ordering which is described later in more detail.
} 
The fulfillment of the stability condition is crucial for the interpretation of the estimated impulseresponse functions (IRFs) and the forecast error variance decomposition (FEVD). Hamilton (1994) shows that a time-series VAR is stable if all eigenvalues $(z)$ of matrix $A$ are strictly less than one which implies that these are inside of the unit circle. A stable VAR process is invertible and has a MA $(\infty)$ representation iff

$$
\operatorname{det}\left(I_{N p}-A z\right) \neq 0 \quad \text { for } \quad|z| \leq 1
$$

Further, Blundell and Bond (1998) demonstrate that the GMM estimator suffer from the weak instrument problems when the endogenous variables are non-stationary or even being close to a random walk. As macroeconomic time series are often integrated of order one (I(1)), we test for unit roots across panels for all endogenous variables. We use the Fisher-type Augmented Dickey-Fuller test designed for unbalanced panel data provided by Choi (2001) where the null hypothesis being tested is that all panels contain a unit root. Moreover, we demean the time series to mitigate the crosssectional dependence as suggested by Levin et al. (2002). We run the panel unit root test considering two models: the first with intercept and the second with intercept \& trend for all variables (GDP, GiniMarket, Low10, Top10) using the three different categories (ALL, HFD and LFD countries) as shown in Appendix 1.6. In general, we cannot reject the null hypothesis that our main variables are a unit root process in levels in the three categories. However, the null hypothesis can be rejected in favor of the alternative that at least one panel is stationary when we run the panel unit root test using growth rates for all variables in the three categories. Since we are interested in the response of the levels of all variables rather that the growth rates, the IRFs presented in this paper are the cumulative IRFs which must be interpreted as the effect on the levels and not the growth rates.

Finally, we rely on the notion of Granger causality (Granger 1969) to assess the causal relationship between economic growth and (average and tails) income inequality. The main idea behind this test is whether the past realization of one variable $(x)$ can help to predict another variable $(y)$ conditional on it is own past realizations $(y)$. If this is indeed the case, we say that $x$ Granger cause $y$ and we are able to reject the null hypothesis that $x$ does not Granger-cause $y$.

\subsection{Empirical Results}

Figures 1.3-1.6 illustrate the estimated cumulative IRFs of the PVAR consisting of the following variables $\mathbf{Y}_{i t}=[G D P$, GiniMarket, Low10,Top10]'. More specifically, they show the cumulative IRFs of the endogenous variables to an orthogonal one standard deviation shocks to the growth rate of the real per capita income (GDP), the average market income inequality (GiniMarket), the income share held by the bottom 10\% (Low10) and top 10\% (Top10) for ALL countries (first row), HFD 
countries (second row) and LFD countries (third row), respectively, over a time span of 10 years. ${ }^{15}$ By cumulating the impact over time, Figures 1.31.6 show the effect on the levels, rather than the effect on the growth rates. Confidence intervals were generated using Monte Carlo simulations based on 1000 draws with $90 \%$ probability bands and impulse responses based on Cholesky identification scheme given by Equation (1.2). All variables are ordered from the most 'most exogenous' to the 'most endogenous', $\mathbf{Y}_{i t}=[$ GDP, GiniMarket, Low10,Top 10$]$ '. All endogenous variables react thus contemporaneously to a GDP shock.

Table 1.2 reports the test of over-identifying restrictions of our estimations. As can be seen, the Hansen test of over-identifying restrictions under the null hypothesis that the instrumental variables are not correlated with the residual cannot be rejected, confirming the join validity of the selected instrument set. Table 1.2 provides information about the numbers of lags used as instruments for each estimation. ${ }^{16}$ Moreover, it shows the transformation method used (forward orthogonal deviationFOD) to remove country-fixed effects, and information about model stability which implies that Equation (1.3) is satisfied.

Table 1.2: Estimation results

\begin{tabular}{l|c|c|c}
\hline & All countries & High FD countries & Low FD countries \\
\hline Number of lags & 1 & 1 & 1 \\
Hansen Test $\chi^{2}$-Statistic & 95.16 & 35.04 & 76.01 \\
Pr $>\chi^{2}$ & 0.12 & 0.33 & 0.14 \\
\hline Number of observations & 526 & 215 & 311 \\
Lags used as instruments & $1 / 6$ & $1 / 2$ & $1 / 5$ \\
Model stable & yes & yes & yes \\
Transformation & FOD & FOD & FOD \\
Econometric Method & GMM & GMM & GMM \\
\hline
\end{tabular}

Numbers of lags based on the Bayesian information criteria (BIC) and Akaike information criteria (AIC). Hansen Test $=$ test of instrument over-identification restrictions where a no rejection of the null hypothesis validate the instruments. Forward orthogonal deviation to remove panel-specific fixed effects.

We conducted a variety of robustness to test the sensitivity of our results. As pointed out in

\footnotetext{
${ }^{15}$ As previously mentioned, we decided to split our sample into High Financial development (HFD) and Low Financial development (LFD) to evaluate the dynamic relationships at different stages of financial development. HFD countries are those countries above the $70^{\text {th }}$ percentile of the overall average distribution of the Financial Development Index. LFD countries are countries below the $70^{\text {th }}$ percentile of the overall average distribution of the Financial Development Index. See Section 1.3 for a detailed explanation.

${ }^{16}$ The program designed by Abrigo and Love (2016) provides the set of lags used as instruments. For example, 1/4 means that we included the first, second, third and fourth lag of the dependent variable in the model.
} 
Section 1.3, we re-estimated the models using the Gini net income inequality (GiniNet), the Financial Institutions Index (FII) and Financial Markets Index (FMI) independently, and report the corresponding estimations results in Appendix 1.6. Furthermore, we re-estimated the models changing the threshold value from $65^{\text {th }}$ and $75^{\text {th }}$ percentile; we change the identification assumption on ordering: ${ }^{17}$ and we use the KAOPEN index, developed by Chinn and Ito (2008), as another proxy for financial development. ${ }^{18}$ Our estimations results are widely robust with respect to these changes.

\section{Dynamic Responses to GDP Shock}

Figure 1.3 illustrates the cumulative dynamic reactions of the endogenous variables to an orthogonal one standard deviation GDP shock. As shown in the first column of Figure 1.3, the GiniMarket gradually decreases in different magnitudes in all three cases. ${ }^{19}$

For ALL countries, the GiniMarket decreases persistently over time and the decline is significantly different from zero after 10 years following the positive GDP shock. The GiniMarket decreases by $.05 \%$ within the first year, and up to $.1 \% 10$ years after following the GDP shock. Similar responses can be observed in both HFD and LFD countries, where the magnitude of the negative effect of GDP on GiniMarket is stronger in the former than the latter. While the GiniMarket in HFD countries decreases by about .15\%, the GiniMarket in LFD countries decreases by about .06\% after 10 years following the GDP shock. Both responses are marginal significant the first years, after which both become insignificant in about six years. Furthermore, Table 1.3 reports the results of the panel Granger causality test for the effect of GDP on the GiniMarket. It can be shown that we can reject the null hypothesis of Granger non-causality for the effect of GDP on the GiniMarket at the $1 \%$ level for ALL countries and at the $10 \%$ level for both HFD and LFD countries. These findings suggest that higher economic growth decreases average income inequality irrespective of the financial development level. While these results cannot be directly comparable to previous studies, it is also worth noting that these findings are in line with da Silva (2020) who shows that economic growth reduces average income inequality.

The Low10 is also affected to a one standard deviation GDP shock in all three categories as shown in the second column of Figure 1.3, although the response is only statistically significant in HFD countries. In HFD countries, the Low10 increases gradually and significantly over time by about .8\% after nine years. Moreover, the magnitude of the positive effect of GDP on the Low10 is stronger

\footnotetext{
${ }^{17} \mathrm{We}$ assume that GDP and our measures of tails income inequality are contemporaneously endogenous to average income inequality, i.e. $\mathbf{Y}_{i t}=[$ GiniMarket, GDP, Low10, Top 10]'.

${ }^{18}$ These results are also available upon request. In addition, we also re-estimated the models using non-overlapping five-year averaged data to purge business cycle effects. These estimations generally yield similar results to those that are described in this Section, however, they are based on a smaller information set and thus less reliable, particularly when the sample is divided into HFD and LFD countries.

${ }^{19}$ For the same variable the range of the $\mathrm{y}$-axis in all graphs is normalized to facilitate a graphical comparison.
} 
in HFD than in ALL and LFD countries. According to Table 1.3 we can reject the null hypothesis of Granger-non causality for the effect of GDP on the Low10 at the 5\% level in HFD countries, but not in ALL and LFD countries. Finally, a one-time one standard deviation GDP shock decreases the Top10 in all three cases. However, these responses are not significantly different from zero as shown in the last column of Figure 1.3. In addition, Table 1.3 also indicates that there is no effect of GDP on the Top10 since the null hypothesis of Granger-non causality cannot be rejected for all cases.

\section{All Countries}
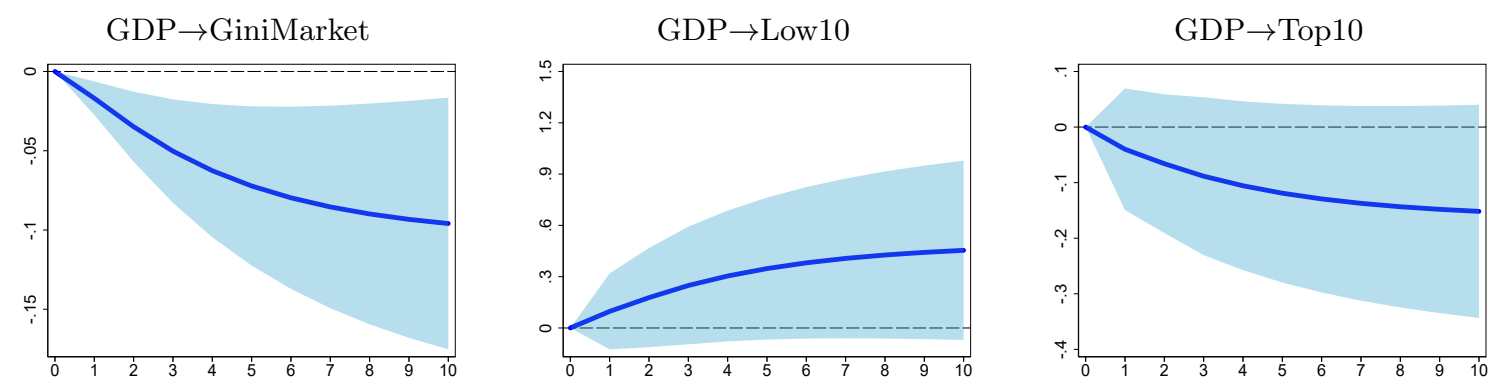

\section{High Financial Development Countries}

GDP $\rightarrow$ GiniMarket

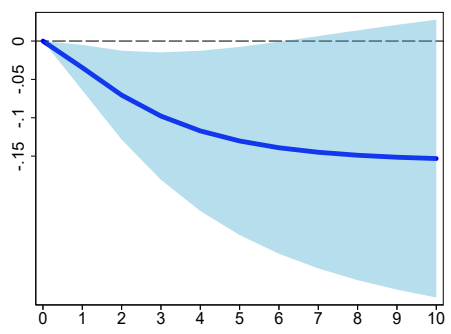

GDP $\rightarrow$ Low 10

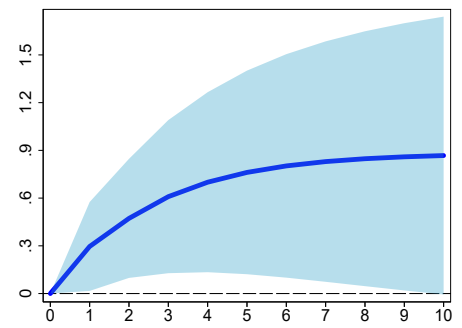

$\mathrm{GDP} \rightarrow \mathrm{Top} 10$

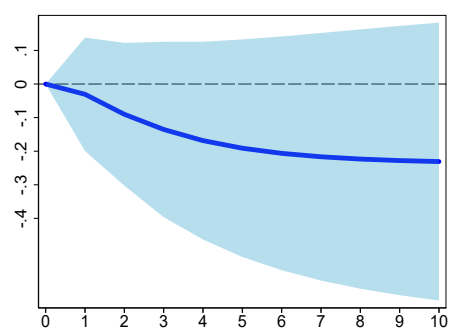

Low Financial Development Countries

GDP $\rightarrow$ GiniMarket

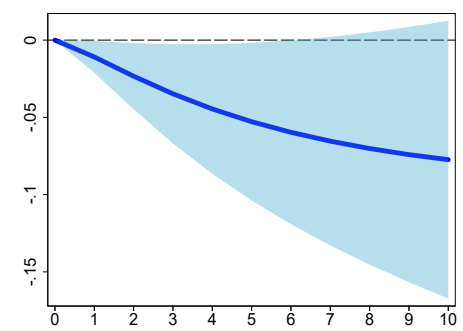

$\mathrm{GDP} \rightarrow$ Low10

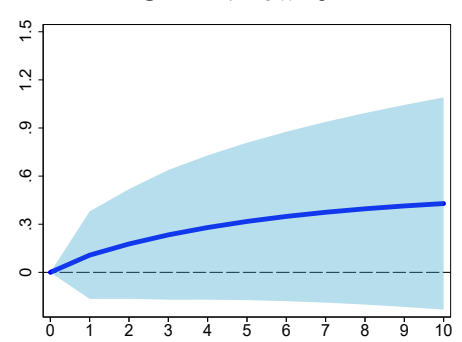

$\mathrm{GDP} \rightarrow \mathrm{Top} 10$

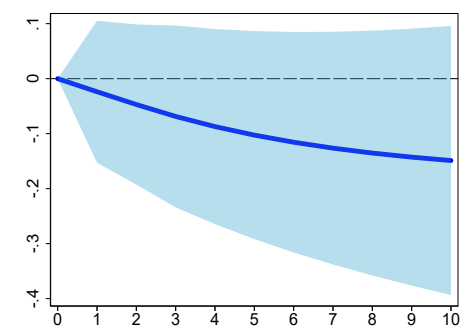

Figure 1.3: Cumulative impulse response functions of the GiniMarket, the Low10 and the Top10 to a positive one-standard deviation shock to the growth rate of real GDP per capita in all countries, High- and Low Financial Development countries. Confidence intervals generated by Monte Carlo simulations based on 1000 draws with $90 \%$ probability bands. Cumulative impulse responses based on Cholesky decomposition, see Equation (1.2). 
Table 1.3: Panel VAR-Granger causality test (GDP $\Rightarrow$ GiniMarket/Low10/Top10)

\begin{tabular}{l|cc|cc|cc}
\hline Variable & \multicolumn{2}{|c|}{ All countries } & \multicolumn{2}{c|}{ High FD } & \multicolumn{2}{c}{ Low FD } \\
\hline & $\chi^{2}$-Statistic & (P-value) & $\chi^{2}$-Statistic & (P-value) & $\chi^{2}$-Statistic & (P-value) \\
\hline GiniMarket & 7.211 & $(0.007)^{* * *}$ & 3.318 & $(0.069)^{*}$ & 2.965 & $(0.085)^{*}$ \\
Low10 & 1.033 & $(0.310)$ & 4.699 & $(0.03)^{* *}$ & 0.820 & $(0.365)$ \\
Top10 & 0.274 & $(0.601)$ & 0.069 & $(0.792)$ & 0.073 & $(0.787)$ \\
\hline
\end{tabular}

${ }^{*} p<0.10,{ }^{* *} p<0.05,{ }^{* * *} p<0.01$ indicate the P-values of the panel VAR-Granger causality test. Null Hypothesis: GDP does not Granger-cause GiniMarket/Low10/Top10. Results based on the estimations presented in Table 1.2

These results suggest that countries with high levels of financial development are more efficient in reducing income inequality since the GiniMarket and the Low10 benefit significantly from higher economic growth. In other words, average and low income groups benefit from higher economic growth when the level of financial development is high and only average income groups when the level of financial development is low. This finding supports the idea of Levine (1997), who stresses the positive effect of a well-functioning financial system on society. The relaxation of credit constraints and the increased accessibility to financial services to those participants who previously were excluded of the financial system may play a fundamental role for the reduction of income inequality, especially for low income groups.

\section{Dynamic Responses to GiniMarket Shock}

Figure 1.4 shows the cumulative dynamic responses of the endogenous variables to an orthogonal one standard deviation GiniMarket shock. A number of results illustrated in these figures are worth highlighting: First, while GDP growth does not react to the other variables contemporaneously by assumption, it reacts with delay in a negative manner to an increase of GiniMarket in all three cases as shown in the first column of Figure 1.4. While the response of ALL countries is significantly different from zero over time, the responses of both HFD and LFD countries are marginally significant the first two years.

For ALL countries, GDP decreases by .06\% within the first year, and up to $3.5 \% 10$ years ahead after the GiniMarket shock. These findings are in line with those of Ostry et al. (2014), Dabla-Norris et al. (2015), Atems and Jones (2015), Jeong and Kim (2018) and Berg et al. (2018), who provide evidence that average income inequality affects negatively economic growth. Table 1.4 indicates that we can reject the null hypothesis of Granger non-causality for the effect of the GiniMarket on GDP at the $5 \%$ level for all countries and at the $10 \%$ level for HFD countries, but not for LFD countries. Moreover, Tables 1.3 and 1.4 suggests that a bi-directional association may exist between GiniMarket 
and GDP but only for countries with high financial development level. These findings do not support previous research such as Atems and Jones (2015) and Jeong and Kim (2018), who argue that the relationship between economic growth and average inequality goes from average inequality to economic growth.

\section{All Countries}
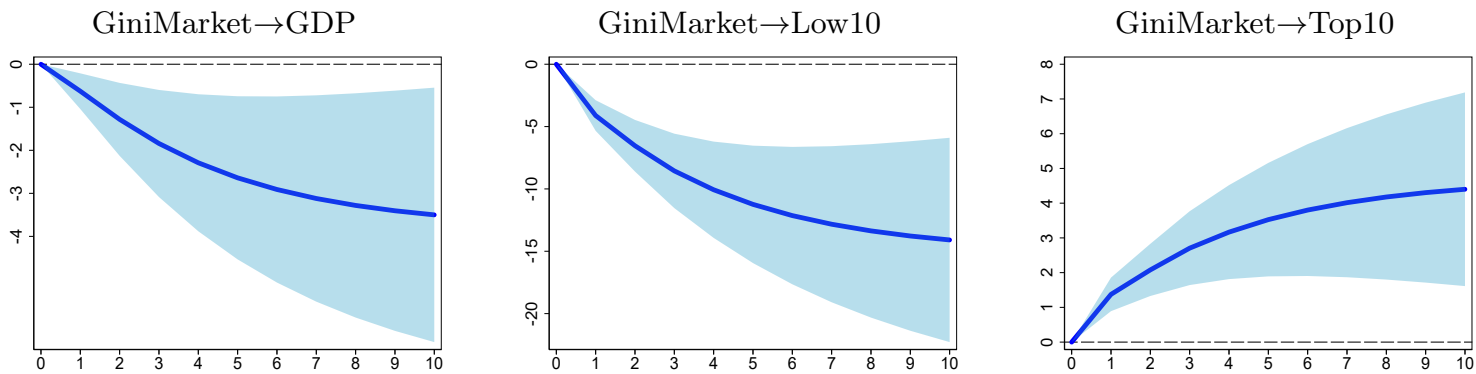

High Financial Development Countries
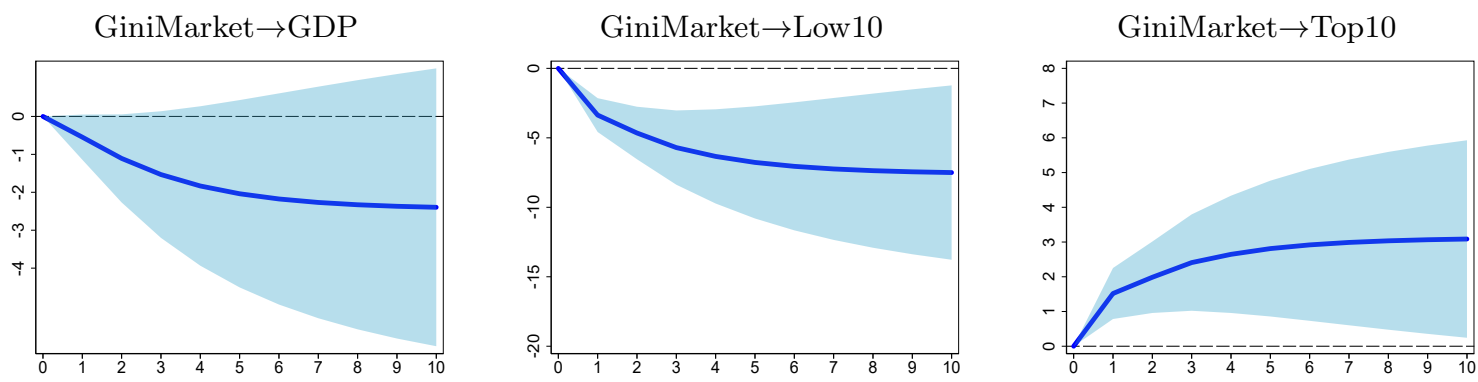

Low Financial Development Countries
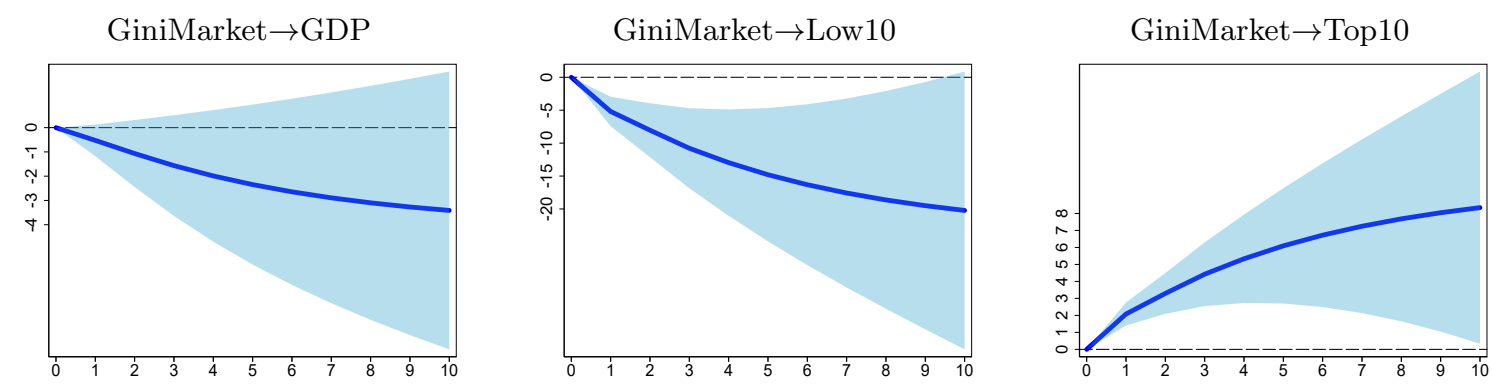

Figure 1.4: Cumulative impulse response functions of the growth rate of real GDP per capita, the Low10 and the Top10 to a positive one-standard deviation shock to GiniMarket in High- and Low Financial Development countries. Confidence intervals generated by Monte Carlo simulations based on 1000 draws with $90 \%$ probability bands. Cumulative impulse responses based on Cholesky decomposition, see Equation (1.2). 
Table 1.4: Panel VAR-Granger causality test (GiniMarket $\Rightarrow$ GDP/Low10/Top10)

\begin{tabular}{l|cc|cc|cc}
\hline Variable & \multicolumn{2}{|c|}{ All countries } & \multicolumn{2}{c|}{ High FD } & \multicolumn{2}{c}{ Low FD } \\
\hline & $\chi^{2}$-Statistic & (P-value) & $\chi^{2}$-Statistic & (P-value) & $\chi^{2}$-Statistic & (P-value) \\
\hline GDP & 5.707 & $(0.017)^{* *}$ & 3.298 & $(0.069)^{*}$ & 1.872 & $(0.171)$ \\
Low10 & 26.430 & $(0.000)^{* * *}$ & 8.734 & $(0.003)^{* * *}$ & 13.036 & $(0.000)^{* * *}$ \\
Top10 & 17.807 & $(0.000)^{* * *}$ & 8.146 & $(0.004)^{* * *}$ & 19.766 & $(0.000)^{* * *}$ \\
\hline
\end{tabular}

${ }^{*} p<0.10,{ }^{* *} p<0.05,{ }^{* *} p<0.01$ indicate the P-values of the panel VAR-Granger causality test. Null Hypothesis: GiniMarket does not Granger-cause GDP/Low10/Top10. Results based on the estimations presented in Table 1.2

Second, an increase of one standard deviation in the orthogonal component of the GiniMarket decreases gradually and significantly the Low10 in all three cases as shown in the second row of Figure 1.4. The strongest negative effect on the Low10 is attributed to LFD countries. The Low10 decreases by $5 \%$ within the first year, and up to $20 \% 10$ years ahead after the GiniMarket shock. Similar reactions are shown in ALL and HFD countries but in a lower magnitude. Table 1.4 displays further support for the effect of the GiniMarket on the Low10 since the null hypothesis can be rejected in al three cases at the $1 \%$ level.

Finally, the last column of Figure 1.4 indicates that the Top10 significantly increases to a GiniMarket shock in all three cases. Similarly, the strongest effect is accounted for LFD countries. While the Top10 in LFD countries increases by 8\%, the Top10 in ALL and HFD countries increases by 3\% and $4 \%$ within the first 10 years after the shock respectively. According to Table 1.4, we can reject the null hypothesis for the effect of the GiniMarket on the Top10 at the $1 \%$ level irrespective of the financial development level of the country. These results highlight the importance of a strong middle income class for a more equitable income distribution within an economy (Kharas, 2010). A higher average income inequality is harmful for economic growth and leads to a more unequal income distribution since low income groups become poorer and high income groups richer irrespective of the country's financial development level.

\section{Dynamic Responses to Low10 Shock}

Figure 1.5 displays the cumulative dynamic responses of the endogenous variables to an orthogonal one standard deviation Low10 shock, which might be related to a "trickle-up" effect. The first two columns illustrate the delay reactions of both GDP and GiniMarket since both variables, by assumption, do not react to the other variables contemporaneously. One the one hand, GDP does not react in a statistically significant manner in any specification, and this is also confirmed by Table 1.5, where the null hypothesis of Granger non-causality for the effect of Low10 on GDP cannot be rejected in all 
All Countries

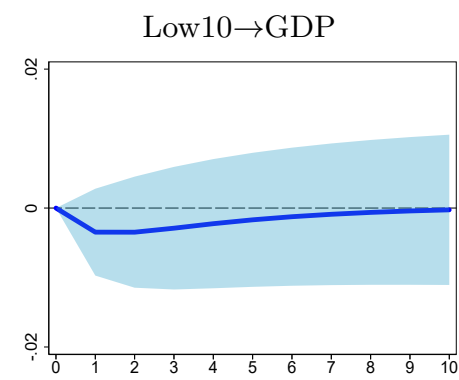

Low $10 \rightarrow$ GiniMarket

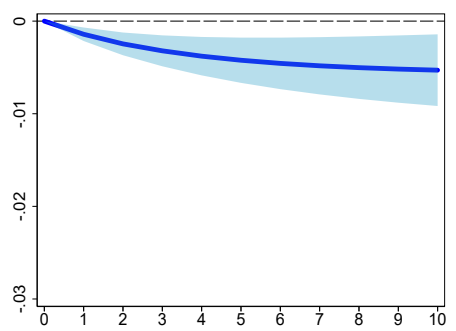

Low $10 \rightarrow$ Top10

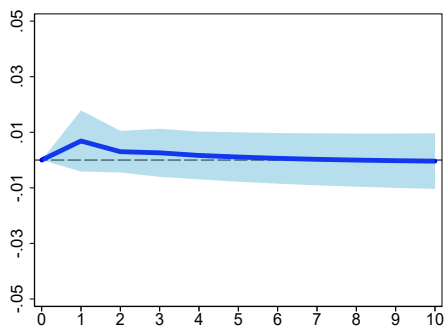

High Financial Development Countries
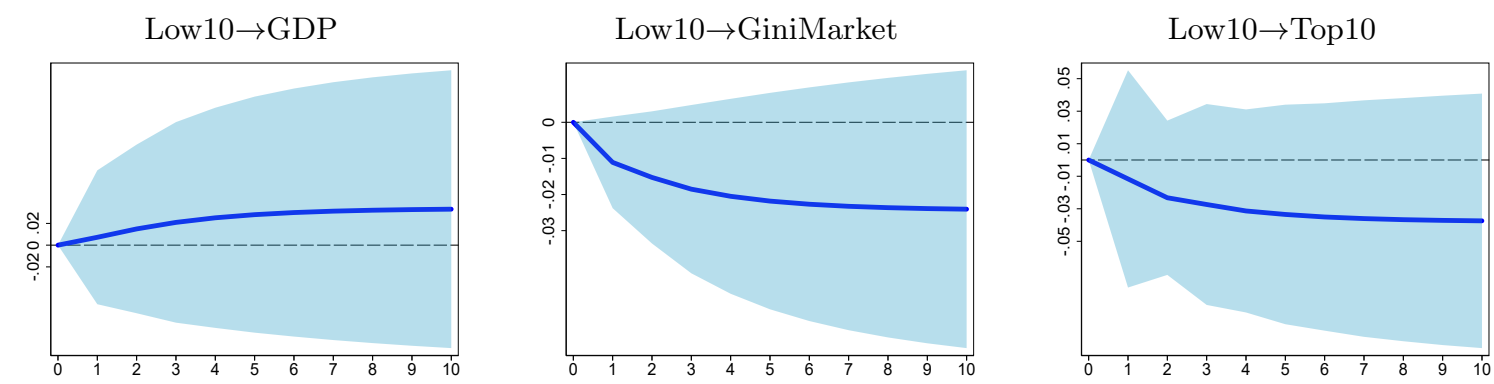

Low Financial Development Countries
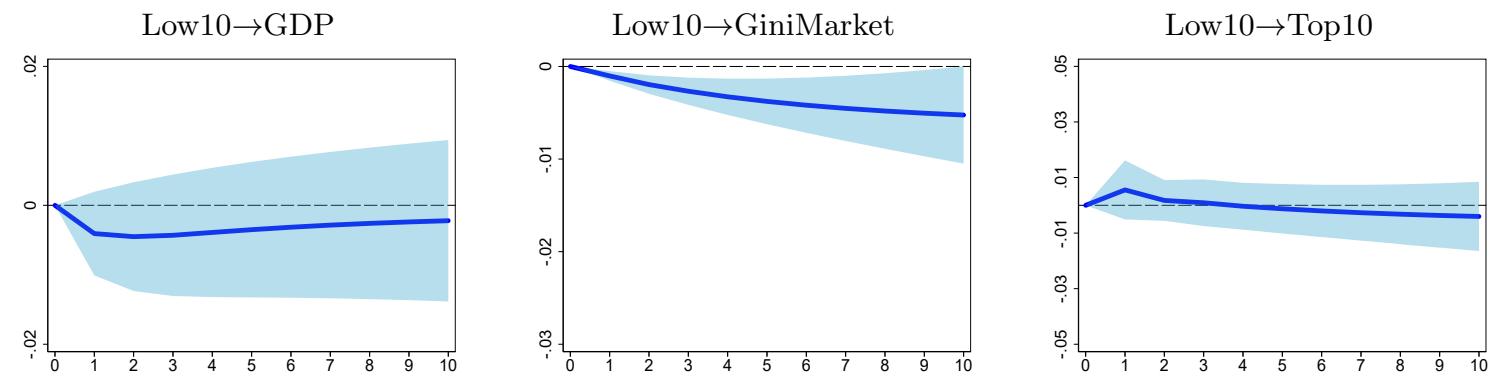

Figure 1.5: Cumulative impulse response functions of the growth rate of real GDP per capita,the GiniMarket and the Top10 to a positive one-standard deviation shock to Low10 in all countries, Highand Low Financial Development countries. Confidence intervals generated by Monte Carlo simulations based on 1000 draws with $90 \%$ probability bands. Cumulative impulse responses based on Cholesky decomposition, see Equation (1.2). 
Table 1.5: Panel VAR-Granger causality test (Low10 $\Rightarrow$ GDP/GiniMarket/Top10)

\begin{tabular}{l|cc|cc|cc}
\hline Variable & \multicolumn{2}{|c|}{ All countries } & \multicolumn{2}{c}{ High FD } & \multicolumn{2}{c}{ Low FD } \\
\hline & $\chi^{2}$-Statistic & $($ P-value) & $\chi^{2}$-Statistic & (P-value) & $\chi^{2}$-Statistic & (P-value) \\
\hline \multicolumn{3}{r}{ Null Hypothesis: Low10 does not Granger-cause GDP/GiniMarket/Top10 } \\
\hline GDP & 0.838 & $(0.360)$ & 1.184 & $(0.277)$ & 1.130 & $(0.288)$ \\
GiniMarket & 12.088 & $(0.001)^{* * *}$ & 2.265 & $(0.132)$ & 13.826 & $(0.000)^{* * *}$ \\
Top10 & 1.070 & $(0.301)$ & 0.001 & $(0.970)$ & 0.931 & $(0.335)$ \\
\hline${ }^{*}<0.10,{ }^{* *} p$ & $<0.05,{ }^{* * *} p<0.01$ indicate the P-values of the panel VAR-Granger causality test. Null \\
Hypothesis: Low10 does not Granger-cause GDP/GiniMarket/Top10. Results based on the estimations presented \\
in Table 1.2
\end{tabular}

On the other hand, the GiniMarket decreases for the three different cases, though to different extents. According to Table 1.5, we can reject the null hypothesis that the Low10 does not Granger cause the GiniMarket for ALL and LFD countries and the 10\% level, but not for HFD countries. Moreover, Tables 1.4 and 1.5 suggest that there may exist a bi-directional relationship between the GiniMarket and the Low10 in LFD countries. This finding suggests that low financial development make average and low income groups more vulnerable and exposed to economic uncertainties. For example, the negative effect of an external shock, such as the COVID-19, will impact differently the society as a whole, being average and low income groups the most affected since they face more credit constraints in countries with a low financial development. By contrast, the mutual interdependence of average and low income groups disappears when financial markets and institutions are more developed. This will mean that the impact of a negative shock will not necessarily lead to a deterioration of average and low income groups simultaneously since any relaxation of credit constraint will, at least partially, compensate the negative effect of any shock. This finding provides further support for the hypothesis that a well-functioning financial system has a positive effect of society.

Furthermore, the last column of Figure 1.5 shows the contemporaneous impact of the Low10 on the Top10 where these responses are not statistically different from zero for all three cases. Moreover, we cannot reject the null of Granger non-causality for Low10 on Top10 irrespective of the financial development level, as shown in Table 1.5. These results provide little support for the existence of a "trickle-up" mechanism since a relative economic improvement of low income groups does not significantly affect neither GDP, nor the Top10 in all three cases.

\section{Dynamic Responses to Top10 Shock}

Figure 1.6 illustrates the cumulative dynamic responses of the endogenous variables to an orthogonal one standard deviation Top10 shock, which might be related to a "trickle-down" effect. By construc- 
tion, Top10 is the 'most endogenous' variable since it responds (theoretically) contemporaneously to all other variables in the model. It is worth noting that while GDP in ALL and LFD countries increases for a long period of time, GDP in HFD countries does not react to a positive Top10 shock. However, these delay responses are statistically insignificant in all three cases. Table 1.6 also indicates that the Top10 does not affect GDP since we cannot reject the null of Granger non-causality for the effect of the Top10 on GDP in all three cases.

By contrast, the GiniMarket and the Low10 react significantly and the effects remain statistically significant over time as shown in the last two columns of Figure 1.6. However, the responses are only significantly for ALL and LFD countries. The GiniMarket decreases gradually over time in both ALL and LFD countries, where the magnitude of the negative impact is stronger in LFD countries. While the GiniMarket in LFD countries decreases by .1\%, the GiniMarket in ALL countries decreases by $.04 \% 10$ years ahead after the shock. Similarly, the strongest positive effect of the Top10 on the Low10 is assigned to LFD countries. The last column of Figure 1.6 shows that the Low10 in LFD countries increases by . $7 \%$ within the first year, and up to $1 \% 10$ years ahead in response to the shock. Table 1.6 reports further support for the effect of the Top10 on the GiniMarket as well as the Low10 since the null of Granger non-causality can be rejected at $1 \%$ level in both cases only for LFD countries, but not for HFD countries.

These findings suggest the existence of a "trickle-down" mechanism for countries with low financial development, in which a higher wealth of income groups benefit the rest of the society. In other words, average and low income groups benefit when high income groups become richer (Aghion and Bolton 1997). Moreover, this may also be explained from the point of view that developing economies have, in general, less competitive markets and few dominant firms. Then, if small elites are the owners of these few dominant firms, and if they experience an increase in their business activities, this will provide more jobs opportunities to middle and low groups. These results may be linked to the works of Acemoglu et al. (2006) and Rioja and Valev (2004) who argue that countries with low levels of financial development follows an investment-based growth strategy. 
All Countries

Top10 $\rightarrow$ GDP

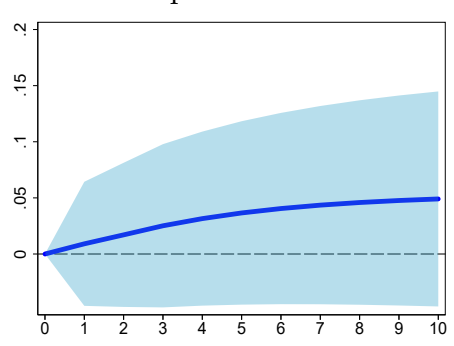

Top $10 \rightarrow$ GiniMarket

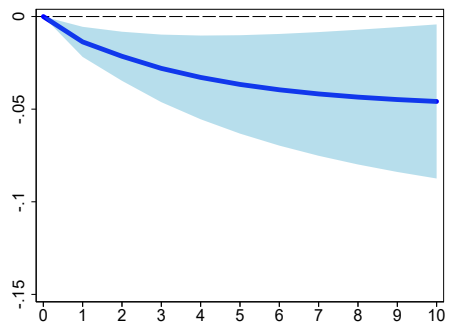

Top $10 \rightarrow$ Low 10

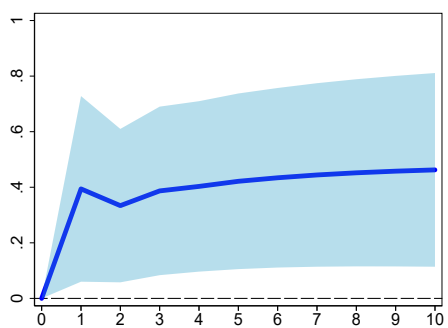

High Financial Development Countries

Top10 $\rightarrow$ GDP

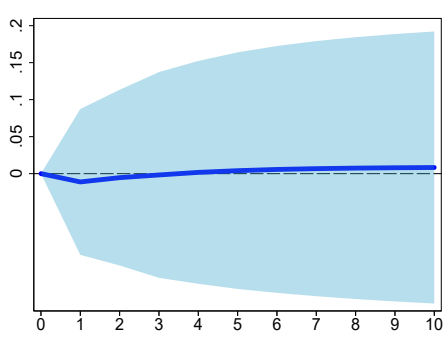

Top $10 \rightarrow$ GiniMarket

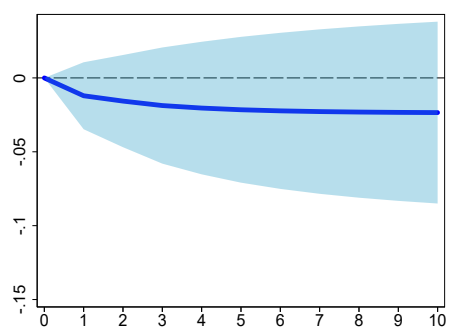

Top $10 \rightarrow$ Low10

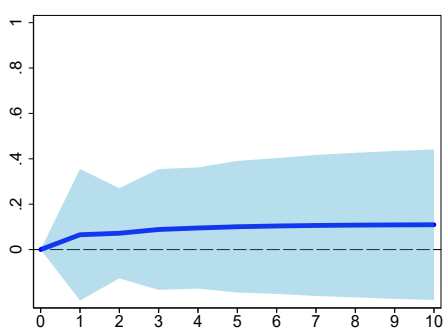

\section{Low Financial Development Countries}

Top $10 \rightarrow$ GDP

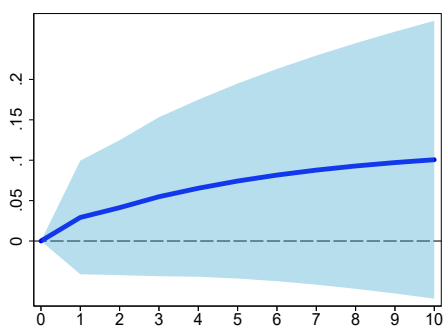

Top10 $\rightarrow$ GiniMarket

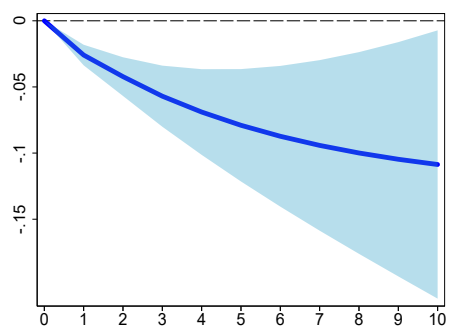

Top $10 \rightarrow$ Low 10

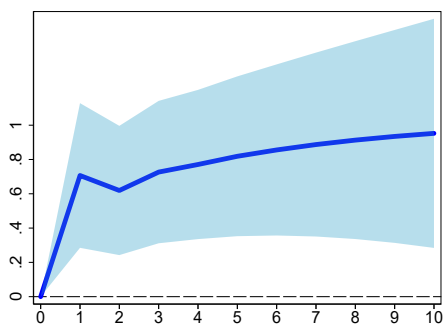

Figure 1.6: Cumulative impulse response functions of the growth rate of real GDP per capita, the GiniMarket and the Low10 to a positive one-standard deviation shock to Top10 in all countries, Highand Low Financial Development countries. Confidence intervals generated by Monte Carlo simulations based on 1000 draws with $90 \%$ probability bands. Cumulative impulse responses based on Cholesky decomposition, see Equation (1.2). 
Table 1.6: Panel VAR-Granger causality test (Top10 $\Rightarrow$ GDP/GiniMarket/Low10)

\begin{tabular}{l|cl|cl|cl}
\hline Variable & \multicolumn{2}{|c|}{ All countries } & \multicolumn{2}{c|}{ High FD } & \multicolumn{2}{c}{ Low FD } \\
\hline & $\chi^{2}$-Statistic & $(\mathrm{P}$-value $)$ & $\chi^{2}$-Statistic & (P-value) & $\chi^{2}$-Statistic & (P-value) \\
\hline GDP & 0.371 & $(0.543)$ & 0.015 & $(0.901)$ & 0.712 & $(0.399)$ \\
GiniMarket & 9.692 & $(0.002)^{* * *}$ & 0.069 & $(0.792)$ & 29.268 & $(0.000)^{* * *}$ \\
Low10 & 4.285 & $(0.038)^{* *}$ & 0.025 & $(0.874)$ & 7.846 & $(0.005)^{* * *}$ \\
\hline
\end{tabular}

${ }^{*} p<0.10,{ }^{* *} p<0.05,{ }^{* * *} p<0.01$ indicate the P-values of the panel VAR-Granger causality test. Null Hypothesis: Top10 does not Granger-cause GDP/GiniMarket/Low10. Results based on the estimations presented in Table 1.2

\section{Forecast Error Variance Decomposition}

Appendix 1.6 shows the FEVD of all estimations presented in Section 1.4. Each row of Figure 1.7 shows the proportion of variation for each variable by each case that is attributed to the shock. The solid line describes the contributions of ALL countries, the dashed line of HFD countries and the dotted line of LFD countries. The first row shows the contributions for GDP shocks in the forecast error variance of the remaining variables. GDP innovations contribute with $10 \%, 7 \%$ and $2.3 \%$ to the forecast error variance of the GiniMarket, the Low10 and the Top10 in HFD countries, respectively. GDP innovations account for a small fraction to the forecast error variance in ALL countries and LFD countries. The second row illustrates the contributions accounted for GiniMarket shocks. While GiniMarket contributions for GDP and the Low 10 are again higher in HFD countries, GiniMarket contributions for the Top10 are higher in LFD countries. For HFD countries, GiniMarket innovations contribute with $7 \%$ and $13 \%$ to the forecast error variance of GDP and the Low10, respectively. For LFD countries, the share of forecast error variance of the Top10 accounted for GiniMarket shock is $20 \%$. Next, the third row illustrates fractions of the forecast variance of GDP, the GiniMarket and the Top10 accounted for innovations of the Low10, being the HFD countries those having the highest contributions. For HFD countries, Low10 innovations contribute with 5\%,1\% and 14\% to GDP, the GiniMarket and the Top10, respectively. Finally, the last row of Figure 1.7 shows that LFD countries are more influenced by the Top10 contributions for the forecast variance of GDP, the GiniMarket and the Low 10 .

In summary, these FEVD deliver additional insights worth highlighting. It can be observed that contributions of the GDP in the forecast error variance of the other variables are the largest in HFD countries. This provides further evidence that countries with high financial development levels are more efficient in reducing inequality. Moreover, Top10 contributions in GDP, GiniMarket and Low10 are the largest in LFD countries. This finding suggests the validity of "trickle-down" effect in which economic policies in favor of large companies and high income groups have a positive effect on lower 
economic classes in society (Aghion and Bolton, 1997).

\subsection{Concluding Remarks}

Although extensive research has provided many insights into the Growth-Inequality nexus, it is still unclear how higher economic growth is distributed across different income groups within a society since previous studies have not treated tails income inequality in much detail. This paper aimed to contribute to the existing literature by analyzing the dynamic relationship between economic growth and (average and tails) income inequality taking into account different stages of financial development using PVARs.

In general, our results suggested that a country's financial development level performs an important function in the Growth-Inequality nexus. Countries with high levels of financial development are more efficient in reducing income inequality. More specifically, average and low income groups benefit from higher economic growth when the level of financial development is high. Moreover, it seems that there is a bi-directional relationship between economic growth and average income inequality, but only for countries with high financial development level. In addition, we showed that a lower Gini coefficient plays a fundamental role for a more equitable income distribution within an economy. More average inequality leads to a more unequal income distribution since low income groups become poorer and high income groups richer irrespective of the country's financial development level. Furthermore, it seems that there is a mutual interdependence between average and low income groups for countries with low levels of financial development. This is, however, not true for countries for high financial development levels where it seems that deterioration of average and/or low income groups will be, at least partially, compensated by the access to financial markets and the relaxation of credit constraints. Moreover, our results suggested the existence of a "trickle-down" mechanism for countries with low financial development, in which a higher wealth of income groups benefit the rest of the society. With other words, average and low income groups benefit when high income groups become richer (Aghion and Bolton, 1997).

Future research with more focus on different measures of tails income inequality is therefore suggested. It may be possible to use the top or bottom $5 \%, 1 \%$ or $0.1 \%$ income shares to have a more clear factual distribution of income inequality within an economy. However, this can be a difficult task due to the insufficient data for income inequality in developing countries. The evolution and progress of new data for income inequality in the near future will hopefully provide a deeper understanding of the drives of income inequality within and across countries. 
1.6 Appendix to Chapter 1 
Country List

\begin{tabular}{|c|c|c|c|}
\hline Albania & Dominican Republic & Lithuania & Sierra Leone \\
\hline Algeria & Ecuador & Madagascar & Slovenia \\
\hline Angola & El Salvador & Malawi & South Africa \\
\hline Argentina & Ethiopia & Malaysia & Spain \\
\hline Armenia & Fiji & Maldives & Sri Lanka \\
\hline Australia & Finland & Mali & St. Lucia \\
\hline Austria & France & Mauritania & Sudan \\
\hline Azerbaijan & Germany & Mexico & Suriname \\
\hline Bangladesh & Ghana & Moldova & Sweden \\
\hline Belgium & Greece & Mongolia & Switzerland \\
\hline Belize & Guatemala & Morocco & Tajikistan \\
\hline Benin & Guinea & Mozambique & Tajikistan \\
\hline Bhutan & Guyana & Myanmar & Tanzania \\
\hline Bolivia & Honduras & Nepal & Thailand \\
\hline Bosnia and Herzegovina & Hungary & Netherlands & Togo \\
\hline Botswana & Iceland & Nicaragua & Trinidad and Tobago \\
\hline Brazil & India & Niger & Tunisia \\
\hline Bulgaria & Indonesia & Nigeria & Turkmenistan \\
\hline Burkina Faso & Ireland & Pakistan & Uganda \\
\hline Burundi & Israel & Panama & United Kingdom \\
\hline Cameroon & Italy & Papua New Guinea & United States \\
\hline Canada & Jamaica & Paraguay & Uruguay \\
\hline Chad & Japan & Peru & Uzbekistan \\
\hline China & Jordan & Philippines & Vietnam \\
\hline Colombia & Kazakhstan & Poland & Zambia \\
\hline Costa Rica & Kenya & Portugal & \\
\hline Cyprus & Latvia & Romania & \\
\hline Czech Republic & Lebanon & Rwanda & \\
\hline Denmark & Lesotho Senegal & Serbia & \\
\hline
\end{tabular}




\section{Variable Definition and Data Source}

\begin{tabular}{lll}
\hline \hline Variable & Definition & Source \\
\hline Gini Coefficient before taxes & $\begin{array}{l}\text { Index which ranges from 0 (perfect inequality) to 100 } \\
\text { (perfect equality) before government intervention }\end{array}$ & SWIID \\
Gini Coefficient after taxes & $\begin{array}{l}\text { Index which ranges from 0 (perfect inequality) to 100 } \\
\text { (perfect equality) after government intervention }\end{array}$ & SWIID \\
& Income share held by highest 10\% & World Bank \\
Top10 & Income share held by lowest 10\% & World Bank \\
Low10 & GDP per capita (constant 2010US\$) & World Development Indicators \\
Financial Developed Index & Index normalized between 0 (low level of financial & Sahay et al. (2015) \\
& development) and 1 (high level of financial & \\
& development). It captures information about financial \\
& corporations and market capitalization & \\
\hline \hline
\end{tabular}

\section{Summary Statistics}

\begin{tabular}{lccccc}
\hline \hline \multicolumn{1}{c}{ Variable } & Mean & Std. Dev. & Min. & Max. & N \\
\hline GiniMarket & 46.279 & 6.095 & 30.6 & 68.8 & 3677 \\
GiniNet & 37.866 & 8.608 & 19.7 & 60.2 & 3677 \\
Top 10 income share & 31.42 & 7.390 & 19 & 54.2 & 1104 \\
Bottom 10 income share & 2.363 & 1.046 & 0 & 5 & 1104 \\
Log GDP per capita & 8.116 & 1.519 & 4.885 & 11.275 & 5585 \\
Financial Development Index & 0.279 & 0.223 & 0 & 1 & 4290 \\
Financial Intitutions Index & 0.366 & 0.23 & 0 & 1 & 4290 \\
Financial Markets Index & 0.182 & 0.237 & 0 & 1 & 4290 \\
\hline \hline
\end{tabular}




\section{Selection Order Criteria}

\begin{tabular}{cccc}
\hline \hline Lag & BIC & AIC & QIC \\
\hline \multicolumn{4}{c}{ All countries } \\
1 & -357.18 & -67.84 & -183.96 \\
2 & -293.03 & -61.55 & -154.45 \\
3 & -221.71 & -48.10 & -117.77 \\
4 & -151.38 & -35.64 & -82.09 \\
5 & -76.14 & -18.27 & -41.50 \\
\hline \multicolumn{4}{c}{ HFD countries } \\
1 & -275.90 & -77.52 & -157.65 \\
2 & -233.95 & -76.27 & -139.96 \\
3 & -171.33 & -54.34 & -101.60 \\
4 & -110.54 & -34.25 & -65.07 \\
5 & -50.49 & -14.88 & -29.26 \\
\hline \multicolumn{4}{c}{ LFD countries } \\
1 & -323.86 & -67.99 & -171.72 \\
2 & -269.21 & -64.51 & -147.50 \\
3 & -208.28 & -54.75 & -117.00 \\
4 & -141.86 & -39.51 & -81.01 \\
5 & -69.90 & -18.72 & -39.47 \\
\hline \hline
\end{tabular}




\section{Forecast Error Variance Decomposition}

\section{GDP contributions}
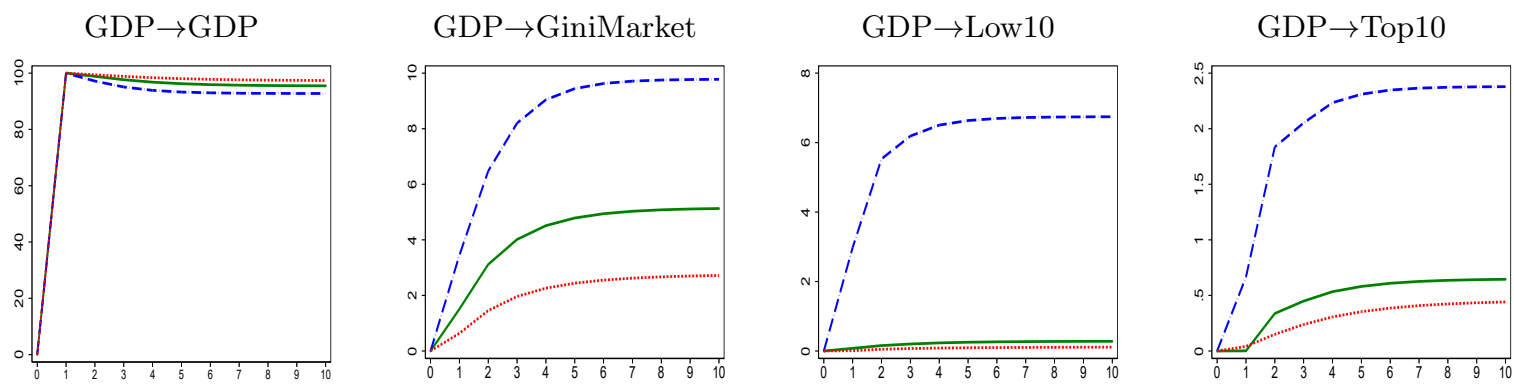

\section{GiniMarket contributions}

GiniMarket $\rightarrow$ GDP

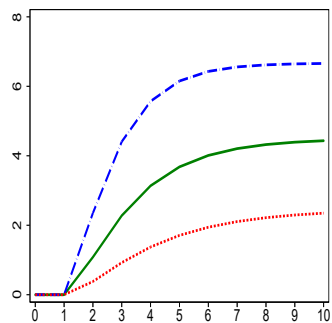

Low $10 \rightarrow$ GDP

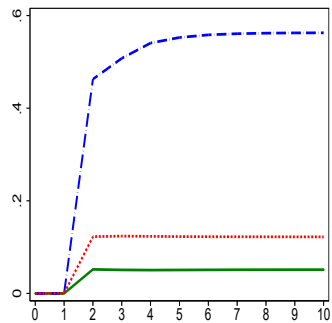

Top10 $\rightarrow$ GDP

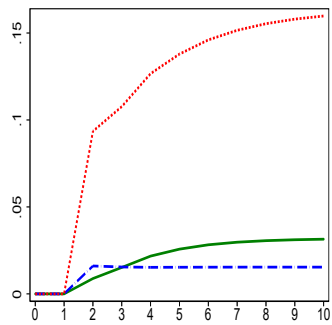

GiniMarket $\rightarrow$ GiniMarket

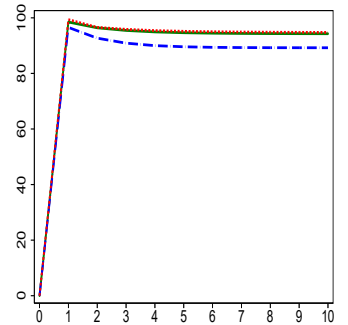

Low10 contributions

Low $10 \rightarrow$ GiniMarket

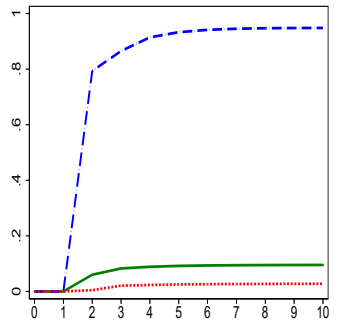

Top10 contributions

Top10 $\rightarrow$ GiniMarket

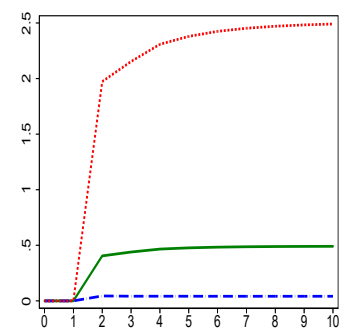

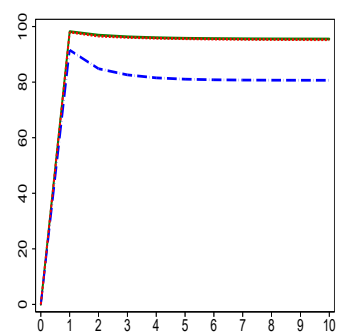

GiniMarket $\rightarrow$ Low10

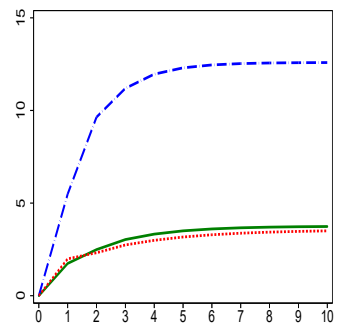

GiniMarket $\rightarrow$ Top10

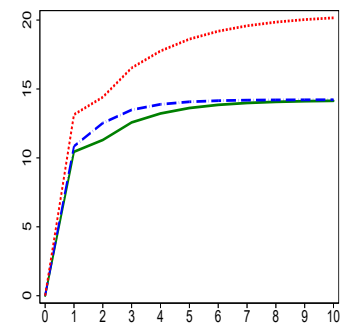

Low $10 \rightarrow$ Top10

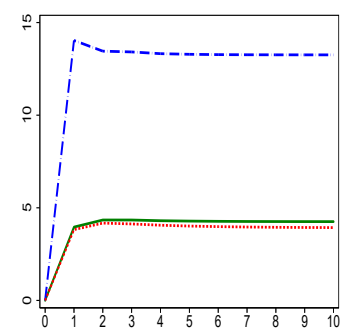

Top $10 \rightarrow$ Top 10
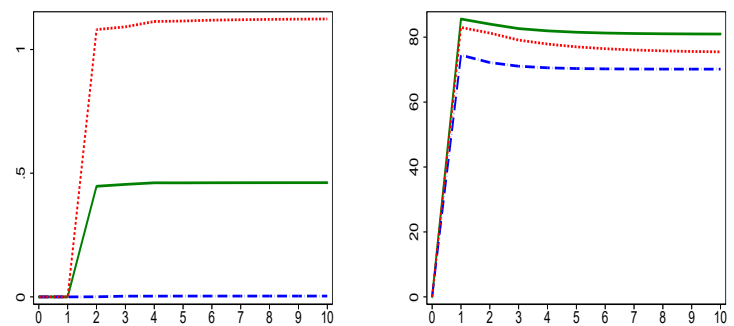

Figure 1.7: Variance decomposition of the growth rate of real GDP per capita, the GiniMarket, the Low10 and the Top10 for ALL countries (green line), High Financial Development countries (blue line) and Low Financial Development countries (red line). 


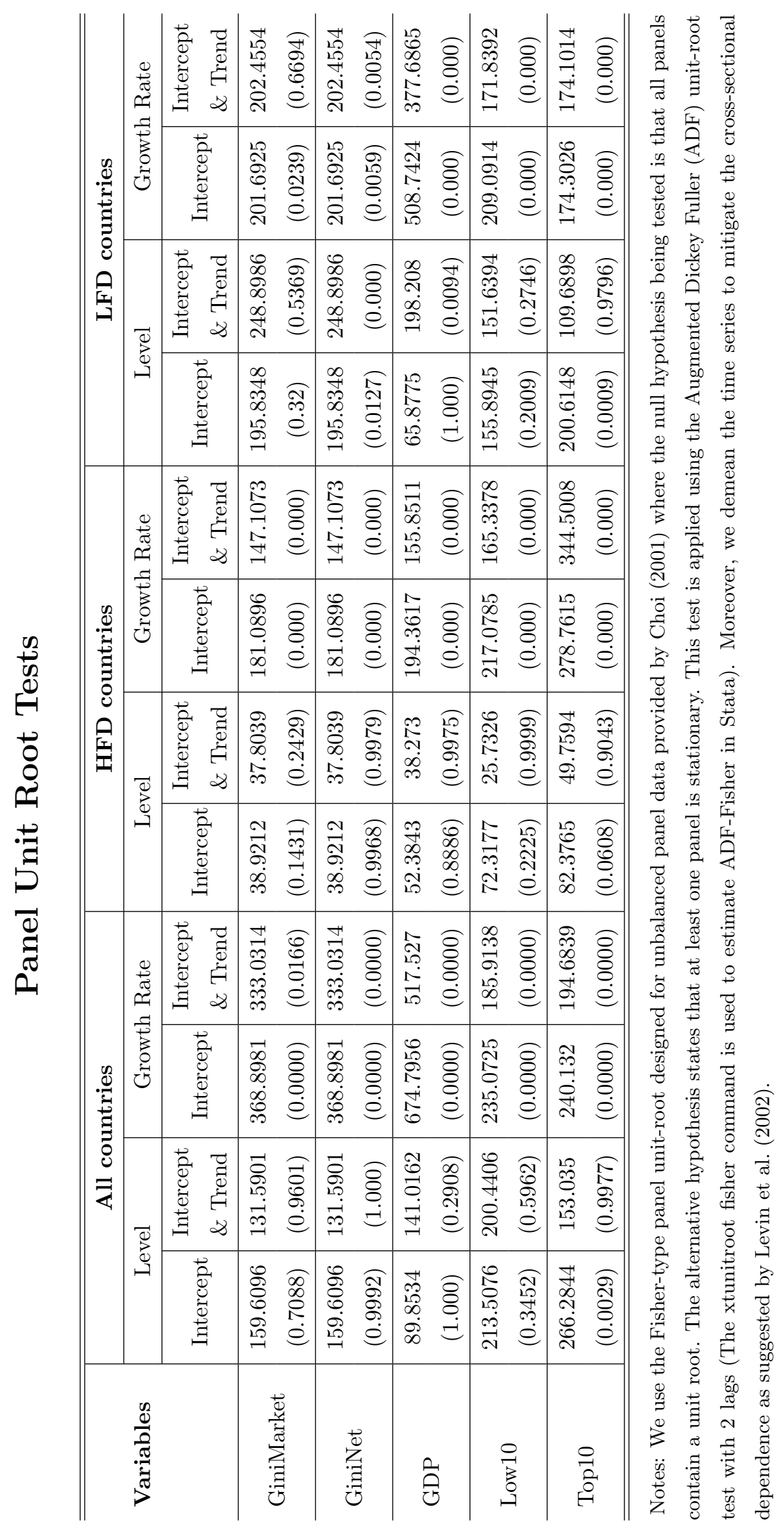




\section{Robustness Check}

\section{Financial Markets Index (FMI)}

Figure 1.8: Cumulative responses using the Financial Markets Index.

\section{Dynamic Responses to GDP shock}

High Financial Development Countries

GDP $\rightarrow$ GiniMarket

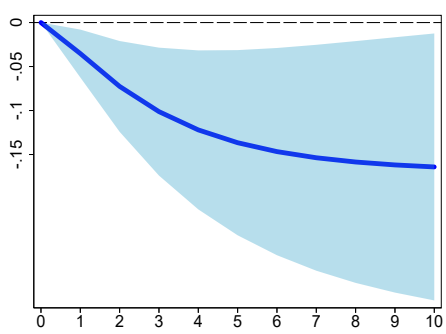

$\mathrm{GDP} \rightarrow$ Low10

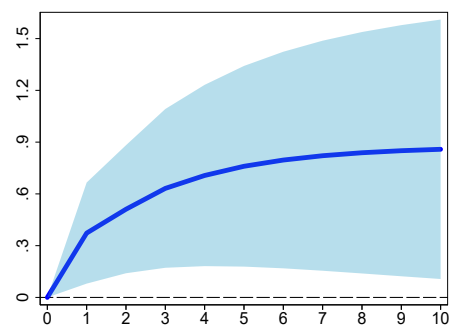

$\mathrm{GDP} \rightarrow \mathrm{Top} 10$

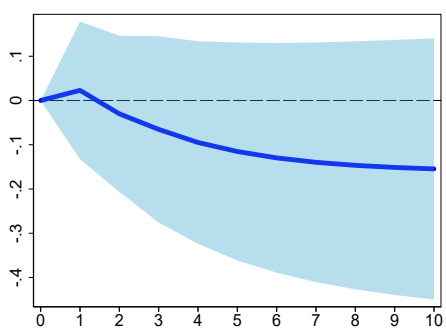

Low Financial Development Countries

$\mathrm{GDP} \rightarrow$ GiniMarket

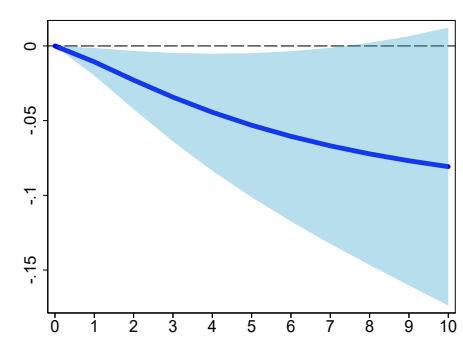

$\mathrm{GDP} \rightarrow \operatorname{Low} 10$

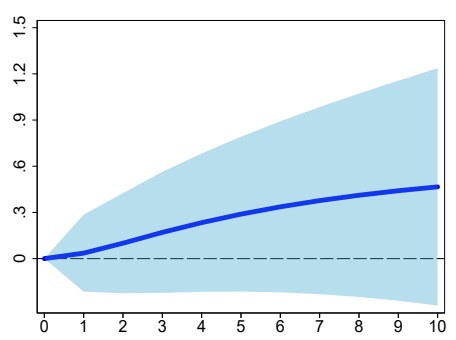

$\mathrm{GDP} \rightarrow \mathrm{Top} 10$

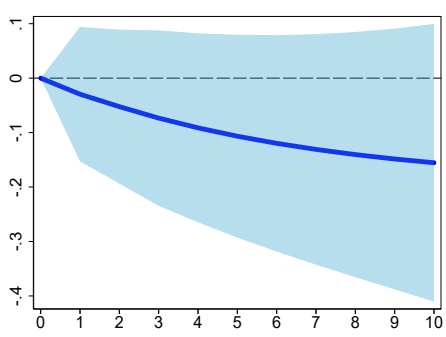

Dynamic Responses to GiniMarket shock

High Financial Development Countries

GiniMarket $\rightarrow$ GDP

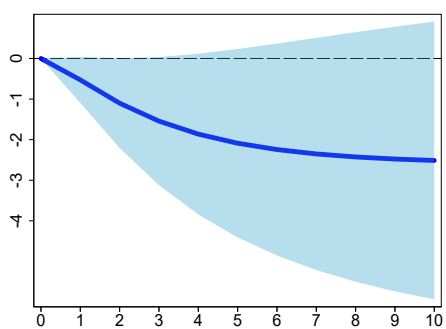

GiniMarket $\rightarrow$ Low 10

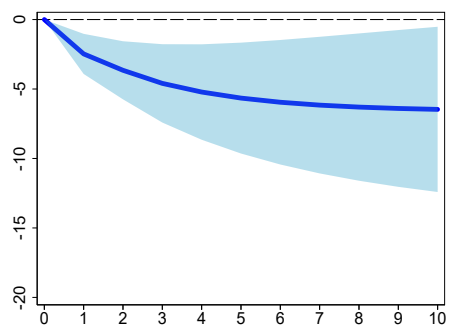

GiniMarket $\rightarrow$ Top10

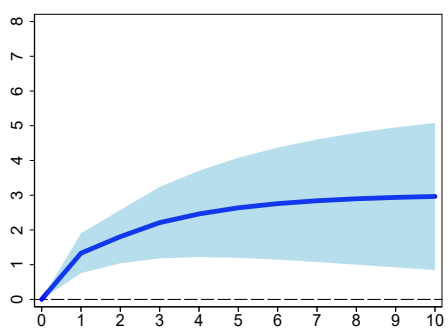

Low Financial Development Countries

GiniMarket $\rightarrow$ GDP

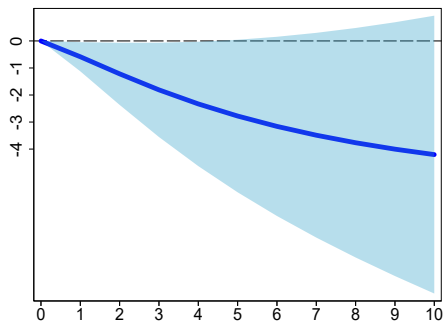

GiniMarket $\rightarrow$ Low10

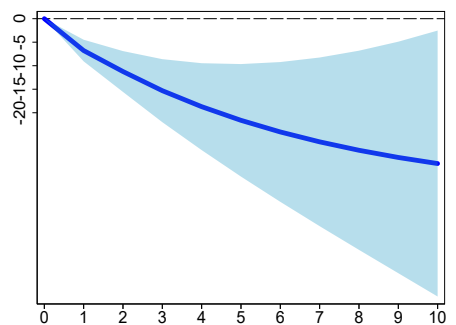

GiniMarket $\rightarrow$ Top10

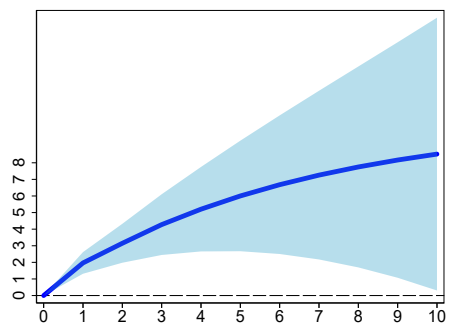




\section{Dynamic Responses to Low10 shock}

High Financial Development Countries
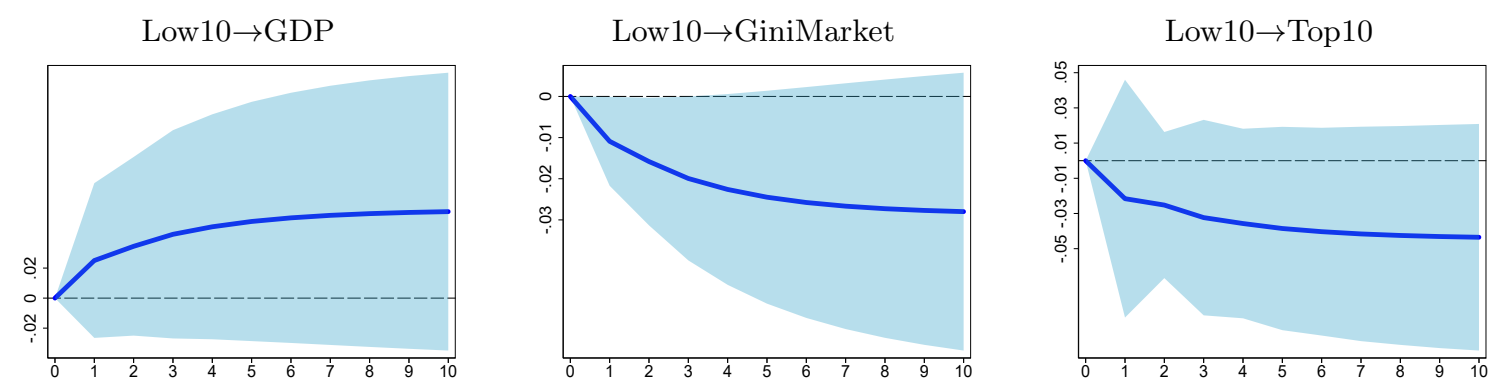

Low10 Financial Development Countries
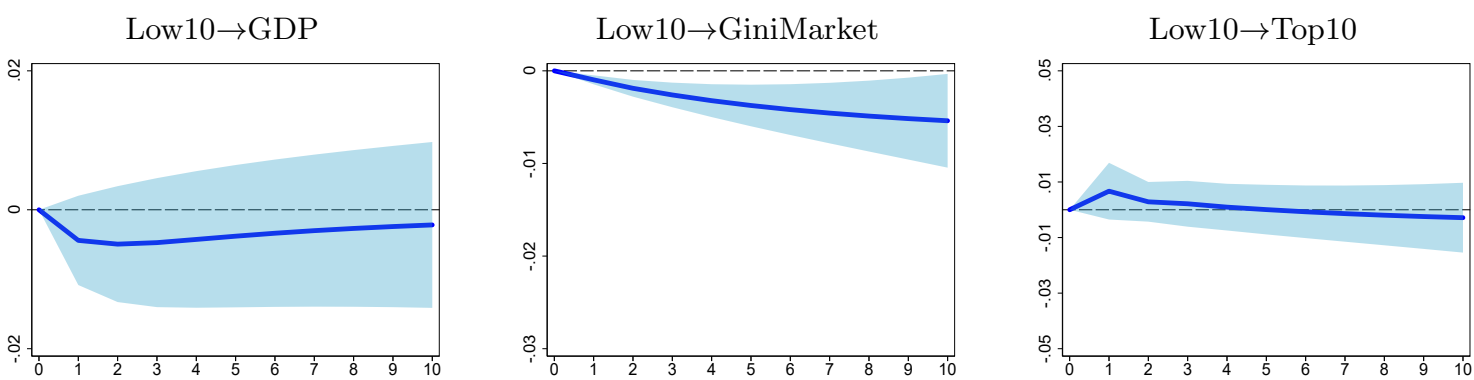

\section{Dynamic Responses to Top10 shock}

High Financial Development Countries
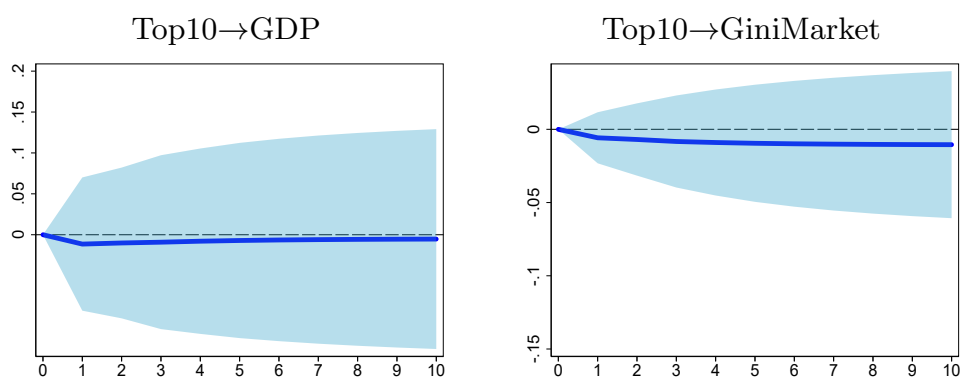

Top $10 \rightarrow$ Low 10

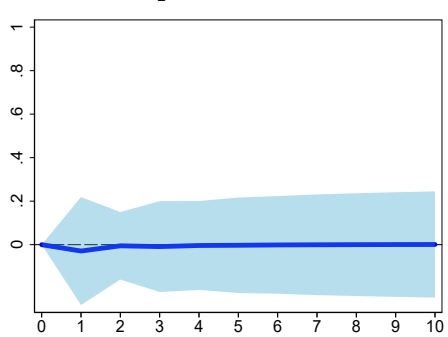

Low Financial Development Countries

Top $10 \rightarrow$ GDP

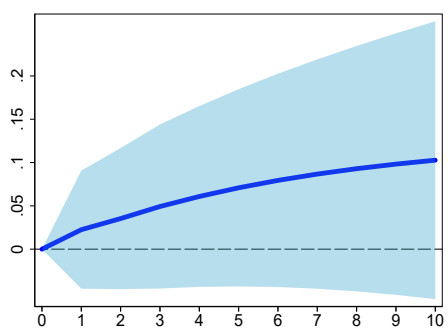

Top $10 \rightarrow$ GiniMarket

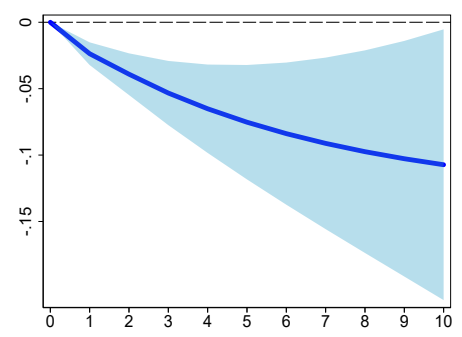

Top $10 \rightarrow$ Low10

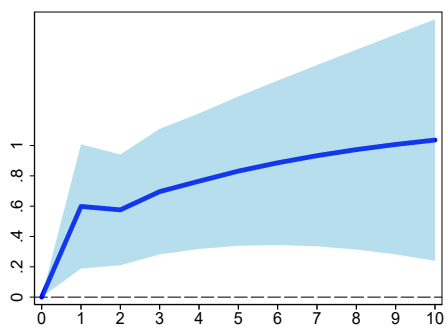




\section{Financial Institutions Index (FII)}

Figure 1.9: Cumulative responses using the Financial Institutions Index.

Dynamic Responses to GDP shock

High Financial Development Countries

GDP $\rightarrow$ GiniMarket

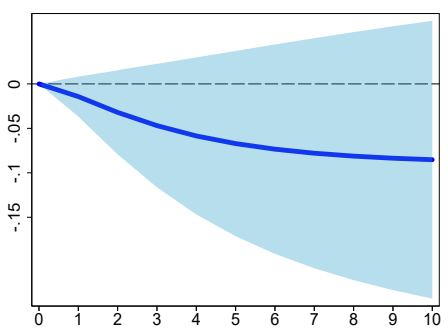

$\mathrm{GDP} \rightarrow$ Low10

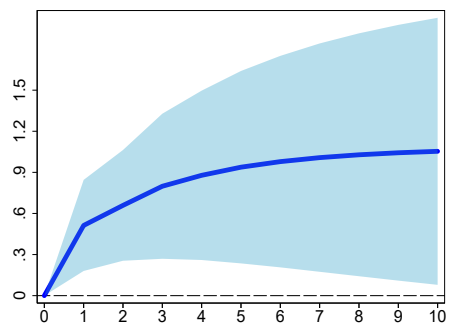

$\mathrm{GDP} \rightarrow \mathrm{Top} 10$

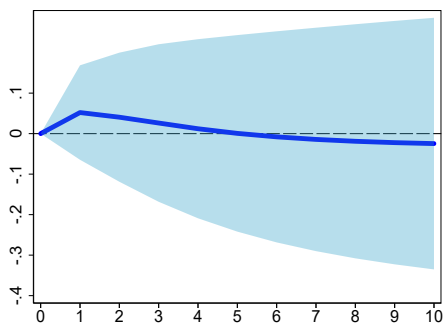

Low Financial Development Countries

$\mathrm{GDP} \rightarrow$ GiniMarket

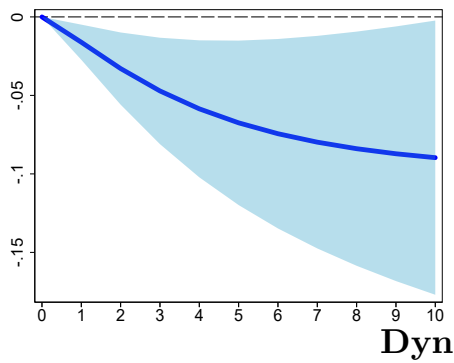

$\mathrm{GDP} \rightarrow$ Low10

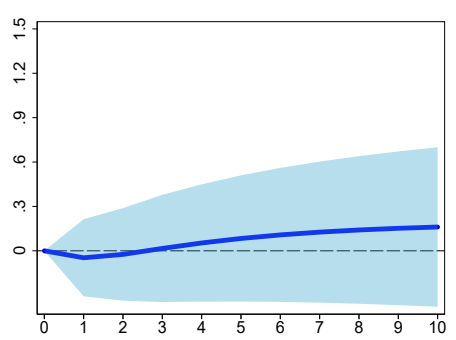

$\mathrm{GDP} \rightarrow \mathrm{Top} 10$

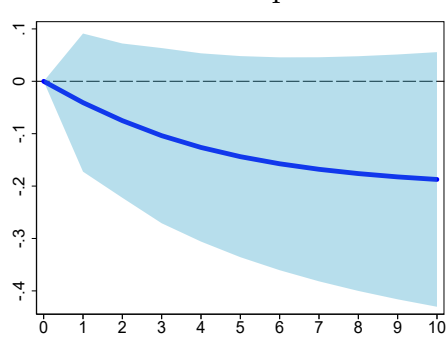

High Financial Development Countries

GiniMarket $\rightarrow$ GDP

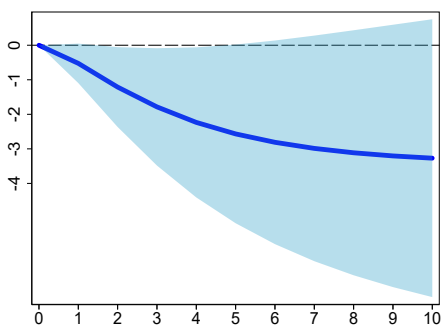

GiniMarket $\rightarrow$ Low 10

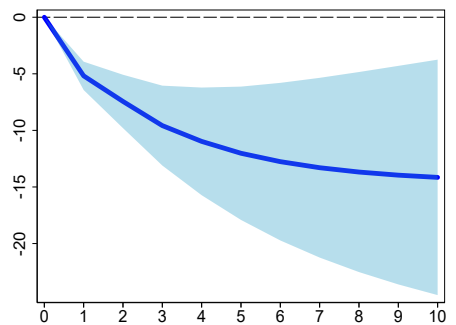

GiniMarket $\rightarrow$ Top10

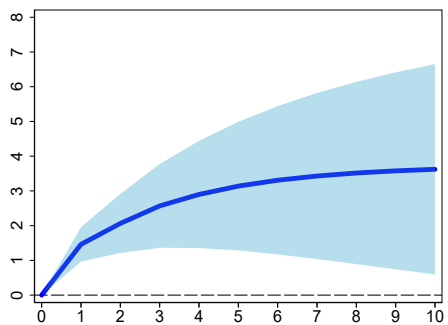

Low Financial Development Countries

GiniMarket $\rightarrow$ GDP

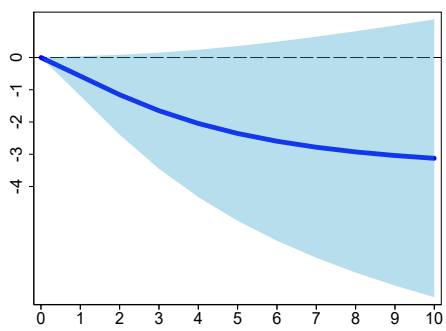

GiniMarket $\rightarrow$ Low10

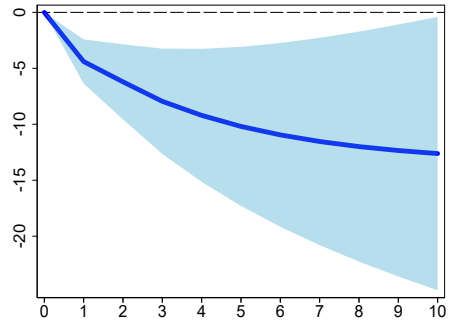

GiniMarket $\rightarrow$ Top10

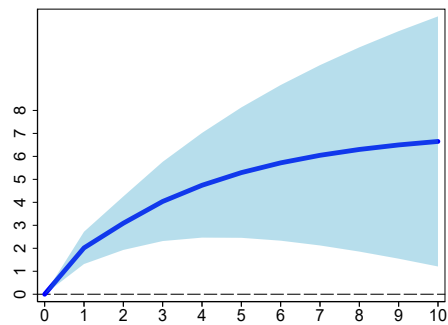




\section{Dynamic Responses to Low10 shock}

High Financial Development Countries

Low $10 \rightarrow$ GDP

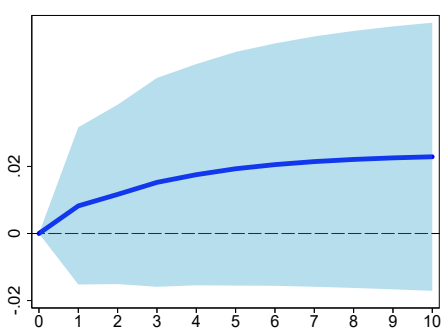

Low $10 \rightarrow$ GiniMarket

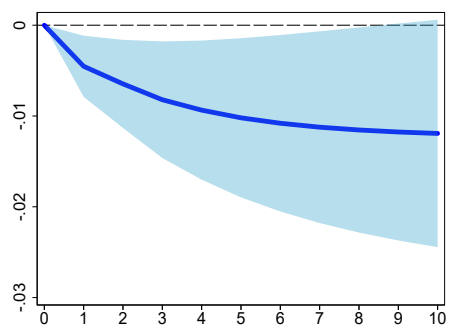

Low $10 \rightarrow$ Top 10

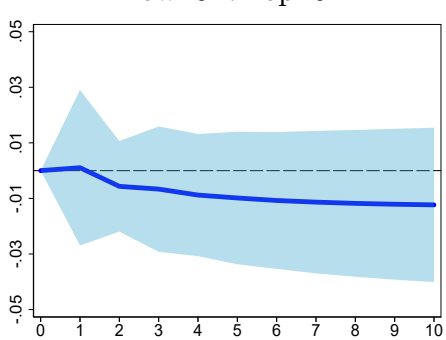

Low10 Financial Development Countries

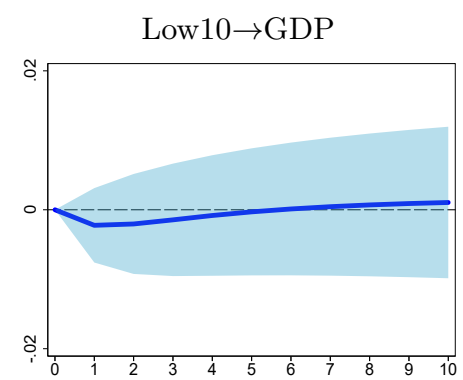

Low $10 \rightarrow$ GiniMarket

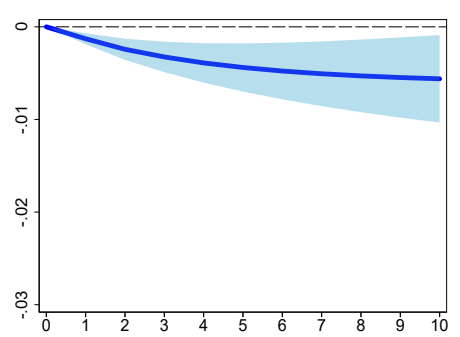

Low $10 \rightarrow$ Top 10

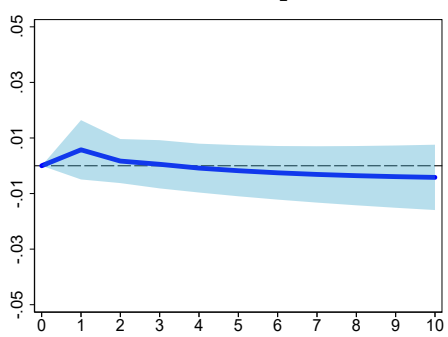

\section{Dynamic Responses to Top10 shock}

High Financial Development Countries

Top10 $\rightarrow$ GDP

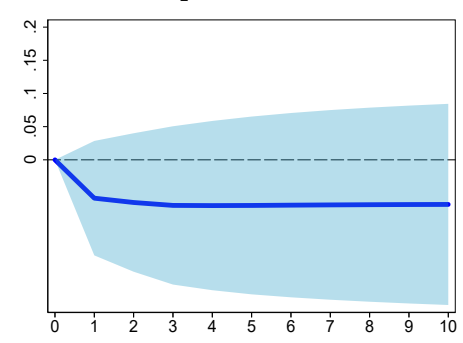

Top10 $\rightarrow$ GiniMarket

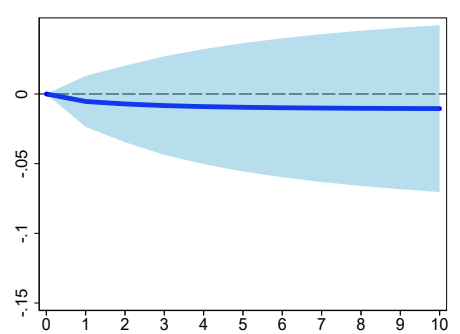

Top $10 \rightarrow$ Low10

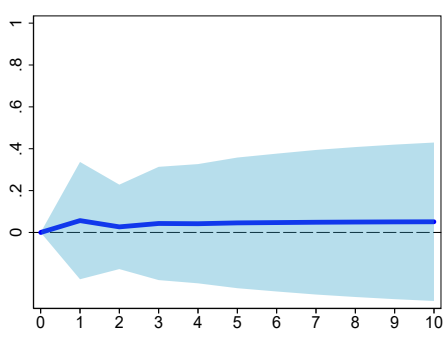

Low Financial Development Countries

Top $10 \rightarrow$ GDP

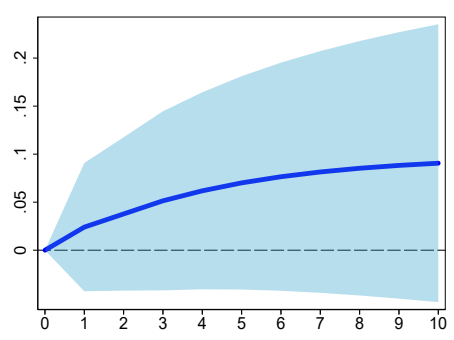

Top10 $\rightarrow$ GiniMarket

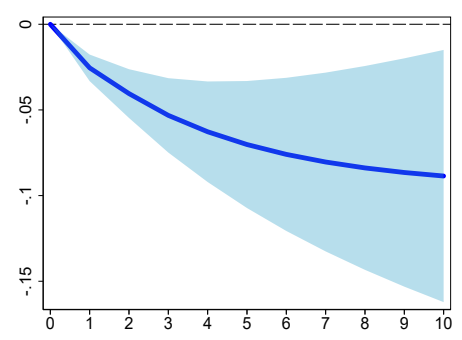

Top10 $\rightarrow$ Low 10

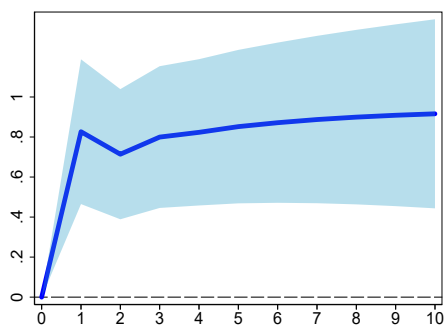




\section{Net Income Inequality (GiniNet) \& Financial Development Index (FDI)}

Figure 1.10: Cumulative responses using using the GiniNet and the Financial Development Index Dynamic Responses to GDP shock

High Financial Development Countries
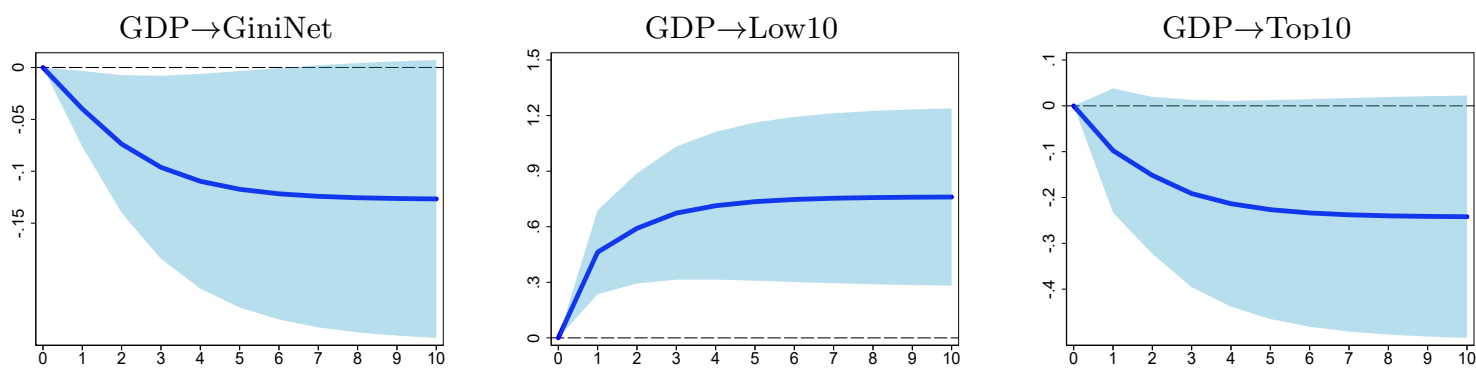

Low Financial Development Countries
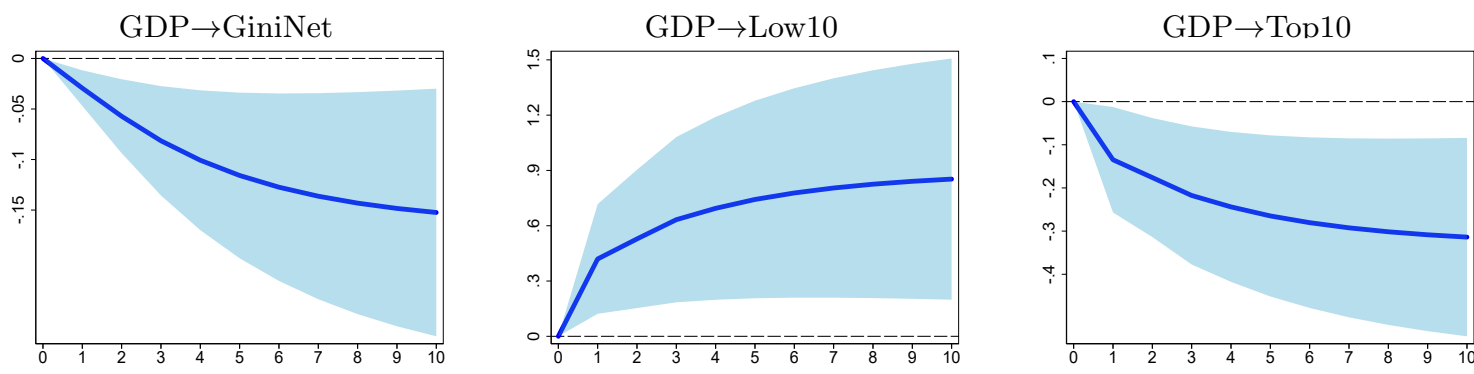

Dynamic Responses to GiniNet shock

High Financial Development Countries

GiniNet $\rightarrow$ GDP

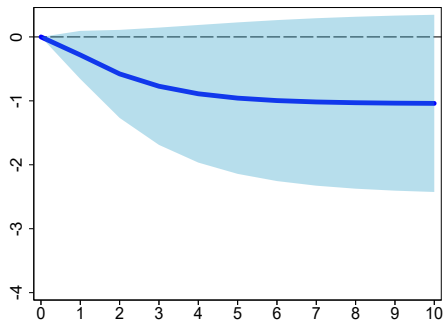

Low Financial Development Countries

GiniNet $\rightarrow$ GDP

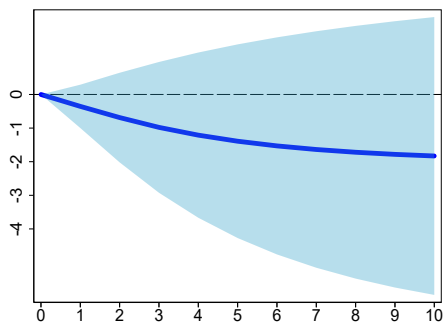

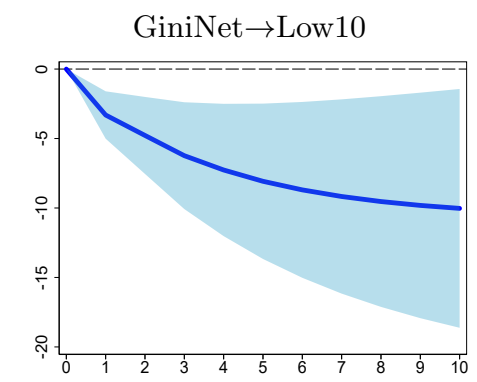

GiniNet $\rightarrow$ Low 10
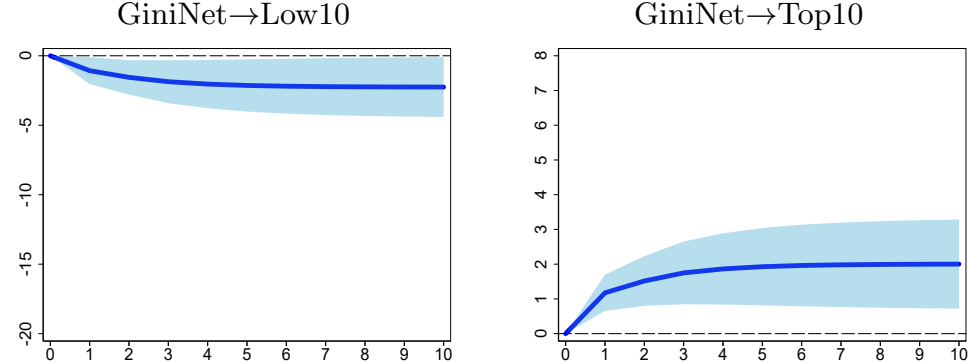

GiniNet $\rightarrow$ Top10

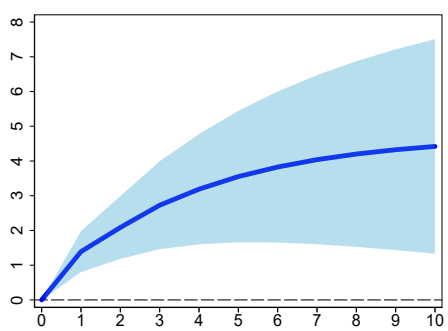




\section{Dynamic Responses to Low10 shock}

High Financial Development Countries

Low10 $\rightarrow$ GDP

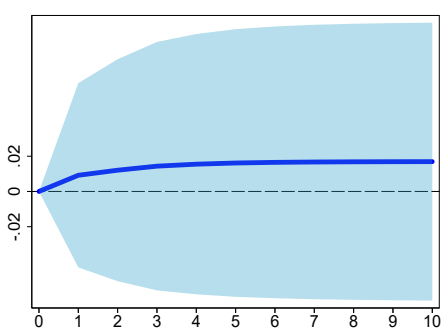

Low $10 \rightarrow$ GiniNet

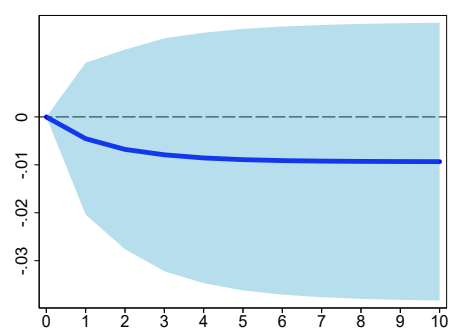

Low $10 \rightarrow$ Top 10

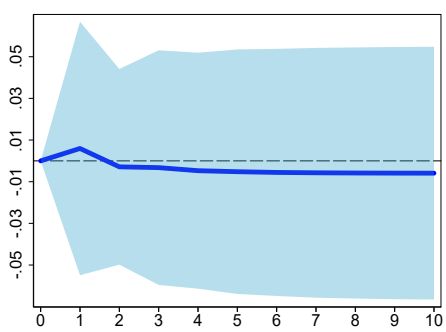

Low10 Financial Development Countries
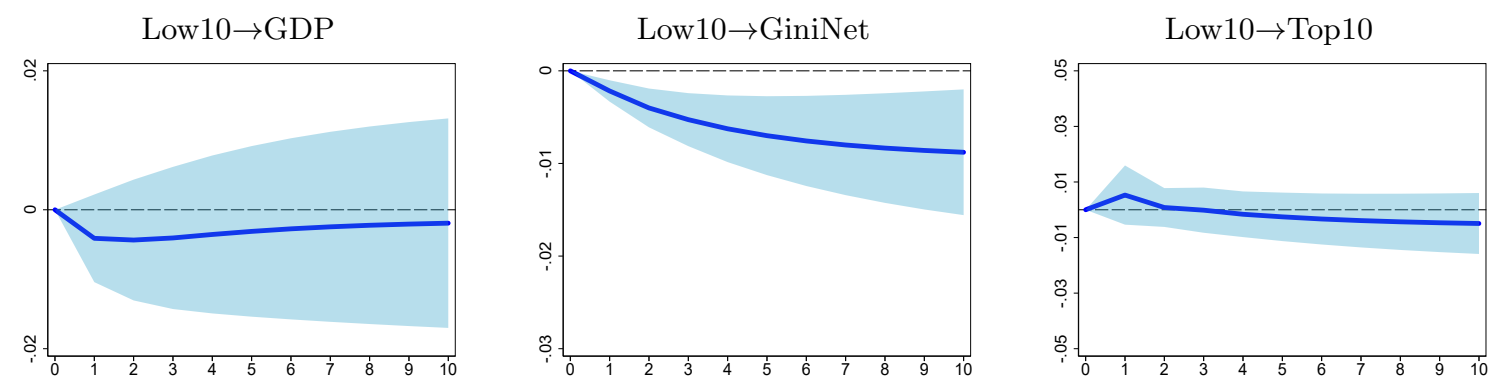

\section{Dynamic Responses to Top10 shock}

High Financial Development Countries

Top10 $\rightarrow$ GDP

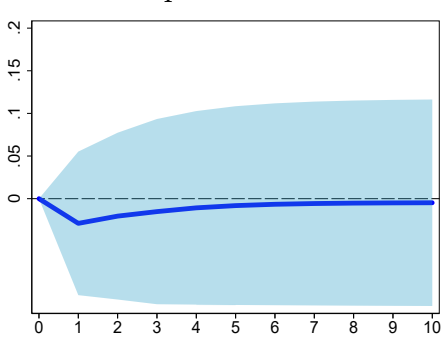

Top10 $\rightarrow$ GiniNet

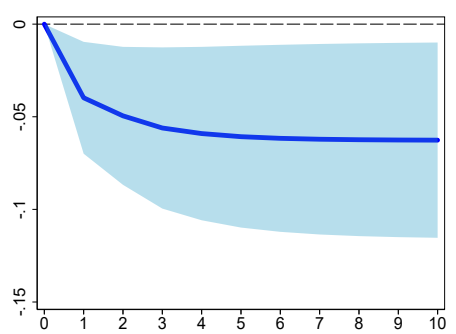

Top $10 \rightarrow$ Low10

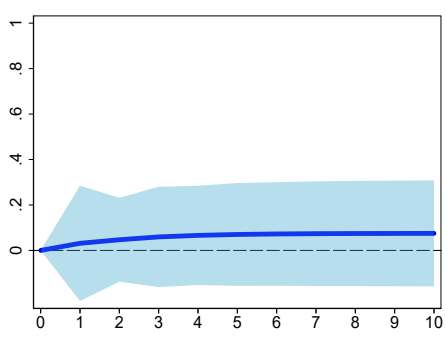

Low Financial Development Countries

Top10 $\rightarrow$ GDP

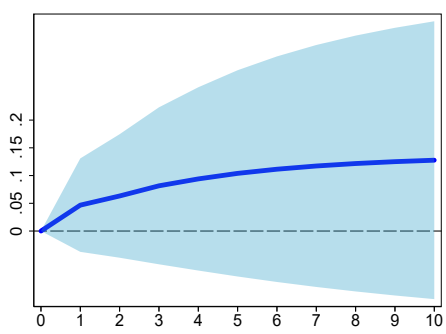

Top10 $\rightarrow$ GiniNet

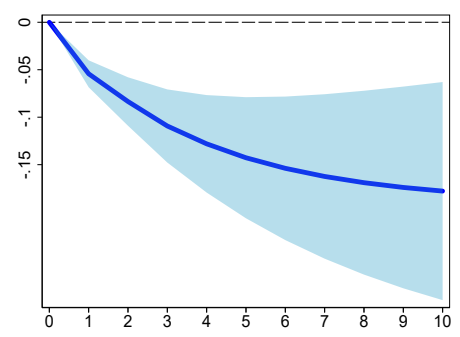

Top $10 \rightarrow$ Low 10

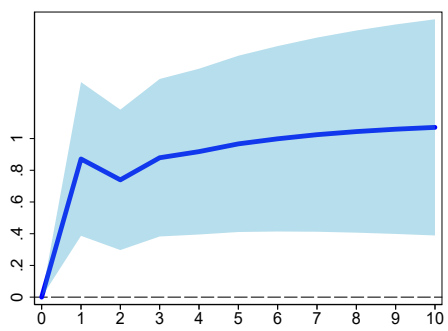




\section{Chapter 2}

\section{Inequality, Macroeconomic Performance and Political Polarization: A Panel Analysis of 20 Advanced Democracies ${ }^{1}$}

\footnotetext{
${ }^{1}$ This Chapter is based on joint work with Prof. Dr. Christian R. Proaño and Prof. Dr. Thomas Saalfeld. Due to suggestions of anonymous referees, this paper reflects the state of research up to January 2021. Earlier versions of this paper can be found in BERG Working Paper No. 157 and BERG Working Paper No. 149.
} 


\section{$2.1 \quad$ Introduction}

The increasing electoral success for radical and populist parties on the left and right of the political spectrum to levels not witnessed since the 1960s and 1970s (Duca and Saving, 2016 McCarty, 2019 Bergmann et al., 2020) is certainly one of the defining phenomena of the last decade. This development has been driven both by endogenous processes arising from party competition (Hetherington 2001 Hetherington and Weiler, 2009, Lachat, 2008, Wagner, 2012, Abou-Chadi, 2016) as well as by socioeconomic factors (Anderson and Beramendi, 2012, Arzheimer, 2013, Bornschier and Kriesi, 2013, Mian et al., 2014, Han, 2016, Vlaicu, 2018). Clearly, both dimensions are relevant as there is little doubt that voting behavior is partly driven by voters" "demand" and preferences generated by exogenous processes, and partly by attempts of party leaders and political entrepreneurs on the "supply" side of political competition to mobilize voters.

The present paper seeks to contribute to the scholarly debate on societal and political polarization focusing on the demand side, and in particular on the effects of income inequality at the societal level on aggregate electoral outcomes. We build methodologically and theoretically on the established notion in Political Science of a "macro polity" (Erikson et al., 2002) which relates electoral results and government popularity to macro-level economic variables in a longitudinal design that can be extended to include a cross-national comparative dimension. A special focus of our analysis is the study of the role of income inequality, measured in a number of ways at the level of the entire society, in variations of political polarization. This seems particularly important due the dramatic rise in income and wealth inequality around the world over the last decades (Atkinson et al. 2011 Stiglitz 2013, Piketty, 2014b). Against this background we employ panel models to investigate how average and tail income inequality have influenced the electoral success of far-left, far-right and centrist parties in legislative elections in OECD countries over the last fifty years.

Indeed, an important caveat of more standard survey-based voter studies and work focusing on party strategies is that traditionally both have neglected the variability of macro-level conditions such as the economic situation or income inequality in different societies and over time. Only recently multilevel studies on the determinants of electoral success have demonstrated that, and how, contextual variables such as income inequality at the macro level might affect individual voter support for radical left and right parties. Such recent studies have shown, for example, that radical right-wing parties draw considerable electoral support form voters suffering the most from societal inequality, namely those in lower socio-economic positions (Lubbers et al., 2002, Arzheimer and Carter, 2006, Rydgren, 2012 Werts et al., 2013). At the meso level of organizations, the literature on niche parties has found that the interests of people at the lower levels of the income distribution are often less well represented by mainstream parties (Gilens, 2012, Carnes, 2013, Carnes and Lupu, 2015, Elsässer et al. 2017 O'Grady, 2019), and new far-left or far-right populist parties can be said to exploit that gap. Further 
work in the niche-party paradigm also suggests that popular support for extreme parties may grow or shrink depending on the strategies of more centrist parties (Meguid, 2005). More demand-side oriented studies have further found that voters penalize government parties retrospectively during and after an economic crisis (Dassonneville and Lewis-Beck, 2014 Fraile and Lewis-Beck, 2014), if responsibility can be attributed. In some cases, voters dissatisfied with their economic position abstain or switch their vote to a mainstream opposition party. In others, new parties, especially new populist parties of the left and right, benefit from a crisis (Kriesi, 2014).

The results of such multi-level models (comparing either nations or regions) are however still somewhat inconclusive depending on the data and research design. Han (2016), for example, explores whether income inequality has dissimilar effects on the support for radical right parties for different social groups across a number of countries, and finds that income inequality encourages poorer people to vote for radical right parties, while it discourages more affluent people from doing so, see also Han and Chang (2016). Further, Rooduijn and Burgoon (2018, p. 1746) find that "[r]adical left and right parties are increasingly successful, particularly among those who experience individual economic difficulties" but that this effect depends on national contexts, a macro-level variable. Counter-intuitively, they find that rising inequality in society actually dampens individual voting for a far-left or far-right party. They explain differences between left and right in this context largely with a "risk aversion mechanism" where far-right outsider parties are seen as a comparatively risky choice for middle-class voters fearing to lose out under conditions of economic uncertainty and inexperienced governments, whereas lower-income voters are less likely to see welfarist far-left parties as a risk for their future well-being. By contrast, Engler and Weisstanner (2020 p. 17) find that rising income inequality increases the probability of voting for far-right parties, but this effect is "strongest among individuals with middle incomes and high status" facing the risk of losing social status (rather than income), see also Burgoon et al. (2019). On a different note, Bloise et al. (2019) investigate voting trends in Italian elections in a regional comparison longitudinal study. They explore the role of income inequality, wealth levels and economic conditions on changes in voting patterns at the regional level from 1994 to 2018 and show that both the Lega and Five Star Movement have benefited from the political and economic upheaval of the last year at the expense of mainstream parties.

The focus of this paper on the aggregate level is not to discount valuable insights that can be, and have been, gained from the study of individual voters and their perceptions with so-called multilevel models, but hopes instead to make use of a larger number of countries and a larger window of observation to detect possible variations in the structural relationship between electoral outcomes and macro-level economic variables. We rely on a simplified but sufficient classification of parties according to their locations along a one-dimensional political spectrum, referring to far-left parties as political parties placed to the left of mainstream center-left parties (such as social democrats) expressing scepticism of capitalism and advocating decisive socio-economic redistribution. Parties located to the right of mainstream center-right parties (such as conservatives and Christian democrats), by contrast, 
are classified as far-right parties. Their ideology is frequently "nativist" in character considering "non-native elements (persons and ideas)" as being "fundamentally threatening to the homogeneous nation-state" (Mudde, 2007, p. 19). In both cases, off-centre ideologies can be combined with a strong dose of populism highlighting the struggle of the "good" (native) people who are betrayed by a corrupted and "evil elite" (Hawkins, 2010). Our definition of political polarization is thus based on electoral support for such parties of the extreme left and right. Rather than including a possible second (e.g. cultural) dimension of political conflict (Inglehart and Norris, 2016), our ranking of far-left and far-right parties is based on a generalized socio-economic left-right dimension, not least because our main independent variables are socio-economic in nature and because this dimension has been shown to be the most salient dimension of political conflict and competition in the longer run (Benoit and Laver, 2006).

One of our main findings is that a significant change in the link between income inequality and political polarization appears to have taken place over the last twenty years. Indeed, we find that both average inequality, measured by the post-tax Gini coefficient, and the bottom $10 \%$ income share are statistically associated with the recent success of far-right parties, while the top $10 \%$ or top $20 \%$ incomes shares are not. The link of income inequality and political polarization seems thus to be based on the deterioration of the relative economic position especially of the poorest fraction of the population, although we do not claim that these actually constituted the main source of electoral support for such parties. Furthermore, we do not find any empirical support for the notion that cultural resentment associated with social and economic globalization has led to an increase in the popularity of far-right parties.

The remainder of this paper is organized as follows: We discuss the existing literature on economics, inequality, and political polarization concerning the connection between these phenomena in Section 2.2. The econometric methodology we use in our analysis is described in Section 2.3, as well as the estimation results. Finally, we draw some conclusions from our study in Section 2.4

\subsection{Literature Overview}

The study of the link between macroeconomic indicators such as economic growth, unemployment, inflation, government debt, or income inequality on the one hand, and voting behavior on the other has a long tradition in Political Science and Political Sociology. This body of scholarship has offered a rich discussion of the mechanisms that connect socio-economic conditions in a society with individual voting behavior and collective electoral outcomes. Traditionally studies have typically focused on a single level of analysis. In the following we overview the existing literature on micro-, macro- and cultural level factors. 


\subsubsection{Micro-level Factors}

At the micro level of individual voters, survey-based election studies have assessed the statistical association between respondents' voting intentions and behavior and their evaluations of their personal (egotropic) and the general (sociotropic) economic situation both retrospectively with regard to the recent past and prospectively relating to the future. Lewis-Beck and Stegmaier (2013 p. 370) summarize a large body of evidence accumulated since the 1970s as follows: "sociotropic evaluations overwhelm egotropic ones. The relatively strong impact of sociotropic retrospective evaluations seems equally clear, regardless of whether the democracy is new or old, low-income or high-income. What remains somewhat controversial is the impact of prospective economic voting". The evidence collected by Lewis-Beck and Stegmaier also suggests that voters have little knowledge about the economy (ibid., p. 373-4) and that the extent to which they vote on perceived economic performance is conditioned by political institutions (e.g. the clarity of government responsibility for the economy) and other macro-level factors (see below).

The economic voting literature demonstrates that government parties frequently get punished (rewarded) for bad (good) performance of the economy. The assumed mechanisms are straightforward: Economic downturns may affect the voters' economic well-being and lead them to take one of four actions: (1) they may stick with the government party or parties, because they do not blame them for the downturn or deem any alternatives as being too risky under the circumstances; (2) they may switch from a governing party to a "mainstream" opposition party, the assumed mechanism for electoral accountability in democratic theory; (3) they may abstain; or (4) they may develop a certain amount of resentment against all mainstream parties and the established political elite. Such general disaffection may be exacerbated in times of economic crisis and expressed in street demonstrations (Kriesi, 2014) or in the support for ideologically extreme parties (Kramer, 1971, Kriesi, 2014, Bartels 2014). Mian et al. (2014), for example, demonstrate that, while the vote share of government coalitions decreases during and after a crisis, the vote share of the opposition parties, the fractionalization of the party system, and voter polarization increase. Similarly, Funke et al. (2016) investigate the political aftermath of financial crises using a longitudinal dataset covering 140 years. They find that far-right parties benefit after a financial crisis, increasing their vote share, on average, by $30 \%$. However, they find no similar evidence for increasing support of far-left parties. ${ }^{2}$

The significant increase in income and wealth inequality around the world in recent decades documented by Atkinson et al. (2011), Stiglitz (2013) and Piketty (2014b), among others, has brought this issue to the center of the political debate, and particularly as a possible explanation of the increasing political polarization in many societies. For instance, Solt (2010) demonstrates how income inequality

\footnotetext{
${ }^{2}$ Another important contribution related to the success of far-right parties is the work of de Bromhead et al. (2012) who shows how far-right parties benefit in hard economic periods during the 1920s and 1930s. They define "hard economic periods" as times characterized by contractions of GDP.
} 
can alter the rates of electoral participation between more and less affluent voters. He finds that higher levels of income inequality reduce the electoral participation of poorer people. In a study of the United States, Gilens (2005) finds that the relatively higher rate of electoral participation among the rich results in policy choices that are biased toward their preferences. These findings could largely be replicated for Germany by Elsässer et al. (2017). In addition, it seems that income inequality not only affects the rates of electoral participation, but also the ideological position of political parties. Pontusson and Rueda (2010) find that left-wing parties move more to the left of the political spectrum when income inequality increases. However, the extent to which left-wing parties move to the left depends on the political mobilization of low-income voters. These findings demonstrate how crises and increasing inequality may affect both the emergence of new radical challenger parties and the ideological repositioning of mainstream parties. This literature also provides some plausible potential mechanisms accounting for the macro-level association between income inequality and polarization.

\subsubsection{Macro-level Factors}

With the growing availability of representative surveys covering voting intentions and behavior, macrolevel studies seemed to have become largely a method for historical phsephologists examining, for example, the role socio-economic factors (especially unemployment) in the rise of Nazism in Germany (Falter, 2013). With the growing sophistication of ecological regression models (King et al. 2004) and the interest in macro indicators such as presidential approval ratings or government popularity (Duch and Stevenson, 2006) or "public-mood" measures (Ura and Ellis, 2012 Stimson, 2018), the interest in macro-level models has grown again. Macro-level studies of economic voting have largely confirmed the findings of those at the micro level, with unemployment, inflation, and GDP growth consistently being the most efficient predictors of government popularity and voting intentions. Although studies on the aggregate level are unsuitable for investigating individual sources of government support, they do allow the modelling of longer-term and cumulative effects of crises as well as of cross-national, institutional or diachronic differences. For example, research on the impact of macro-economic conditions on public support for the government shows the importance of cross-national variations in political institutions (Powell et al., 1993, Anderson, 1995, Hellwig and Samuels, 2008) and differentiated levels of exposure to global trade (Hellwig and Samuels, 2007). They also demonstrate that penalties for poor economic performance for incumbent parties generally come on top of a regular electoral cost of governing (Lewis-Beck and Stegmaier, 2013, p. 376-379). Since polarization (rather than voting for extreme parties) is a macro-level indicator, studies located at this level of analysis are particularly pertinent for the present analysis.

Recent studies such as Duca and Saving (2016) document a close relationship between economic inequality and political polarization in the United States at the macro level. Voorheis et al. (2015) corroborate this finding using U.S. state-level and suggest additionally that higher income inequality 
may cause state legislatures move to the right, leading often to a replacement of Democratic legislators by Republican ones. Along these lines McCarty et al. (2016) stress also the effect of economic inequality on the political landscape and argue that the political polarization in American society may be partially explained by the overall course of fiscal policy and the deregulation of the economy since the Reagan administration. By contrast, Iversen and Soskice (2015), using a country-level panel of advanced economies argue that more egalitarian societies - in terms of education and access to information may be more polarized politically. ${ }^{3}$

\subsubsection{Cultural Factors}

In addition to the relevance of economic factors, scholars have also identified globalization and other social processes unleashed by economic modernization as further important determinants of political polarization. Since globalization can be conceptually understood as deeper political, cultural, and economic integration across national borders, it is possible that these processes may also generate considerable changes in societies, which in turn may trigger polarization between a cosmopolitan left benefiting from these processes economically and culturally and a nativist right resentful not only of being left behind economically but also about a loss of cultural identity and social recognition. Thus, some authors have emphasized the possible role of a "cultural backlash" (Inglehart and Norris, 2016) and produced an influential thesis focusing on the "losers of modernization" (Betz 1994) to explain the growing popularity of far-left/right parties in recent decades.

According to the cultural backlash thesis, Western societies have shifted toward more post-materialist values since the 1970s (the so-called "silent revolution"). These cultural transformations are believed to have created defensive reactions among some social groups, especially those holding traditional values, being less educated and older relative to the average population. The "losers of modernization" thesis is similar. It emphasizes the idea that some groups in society will be unable to adapt to the post-industrial processes unleashed by globalization. People with lower levels of education, in particular, are thought to be adversely affected by these transformations. Consequently, political conflict is hypothesized to be triggered by the fact that these groups feel that they are not sufficiently represented through the mainstream parties. Far-right parties have benefited from this situation, commonly leading to an increase in nativist sentiments, accompanied by anti-immigration and (in the European Union) anti-EU attitudes.

Some empirical research has concluded that cultural backgrounds may play a key role in voting for populist parties (Inglehart and Norris, 2016). However, scholars disagree about the linkage between far-right parties and the cultural context. While Knigge (1998) and Swank and Betz (2003) find a

\footnotetext{
${ }^{3}$ As we will discuss below, this finding are in stark contrast to our results. It should be noted, however, that their measure of polarization is based on the aggregation of subjective responses from correspondents in each of the countries they study, while our measure is based on the share of seats in the parliament occupied by far-left and far-right countries.
} 
positive relationship between far-right parties and the level of immigration, Dülmer and Klein (2005) and Rydgren (2008) are unable to find any statistical relationship between them.

In sum, there is a theoretically and empirically rich body of scholarship on the link between social inequality and the perception of absolute or relative deprivation on the one hand and voting for far-left or far-right parties on the other. At the aggregate level, the link between economic performance and social inequality on the one hand and success of ideologically extreme parties at one end - or (as in the German Weimar Republic, 1919-1933) both ends - of the political spectrum on the other still requires further research. While recent multi-level studies have proposed some plausible mechanisms connecting variations in social inequality to polarization as an outcome, they have done so under a number of assumptions that we are proposing to put to a test. One crucial assumption is that the effect of social inequality on polarization is invariate across time. By using a relatively long time series across a number of countries, we will be able to account for any potential changes in this respect. The econometric methodology employed in this paper, as well as the data, and the results are described in the next section.

\section{$2.3 \quad$ Empirical Results}

\subsubsection{Data Description}

For our empirical analysis, we use panel data for 20 advanced countries on an annual basis ranging from 1970 to 2016. Our dependent variables are based on the outcomes of parliamentary elections. For this purpose, we use the Parliaments and Governments Database by Döring and Manow (2015), which provides extensive coverage of general elections in several democratic countries. We calculate the vote share of far-left parties, the vote share of far-right parties, and the vote share of the remaining (mainstream)parties in each parliamentary election for all countries in our sample. ${ }^{4}$ In total, we collect 291 parliamentary elections throughout 1970-2016. A list of all parliamentary elections, as well as of all countries analyzed in this paper, can be found in Appendix 2.5

To identify parties according to their ideological position, we follow the party codification by Funke et al. (2016), who analyze the link between political outcomes and financial crises in 20 advanced economies from 1870 to 2014. Accordingly, the far-right vote share (FRVS) is composed of those political parties ranging from right-wing populism to the radical right along the political spectrum. These parties possess not only nationalistic and authoritarian attitudes, but also anti-immigrant sentiments. For example, the National Front in France and the Party for Freedom in the Netherlands belong in this category since they are considered anti-EU political movements and have criticized

\footnotetext{
${ }^{4}$ All national elections analyzed in this paper were held in one particular year, with the exception of Greece, which held two national elections in 2012. In this case, we used the second election from that year.
} 
the EU elite for the uncontrolled flows of migrants and refugees from countries at war into Europe. Similarly, the far-left vote share (FLVS) is calculated by summing up all parliamentary seats of those parties ranging from left-wing populism to the radical left. These parties support greater egalitarianism based on Marxist-Leninist positions, and reject the current international economic order, such as Syriza in Greece and Podemos in Spain. A list of all parties that are categorized as far-left and far-right is given in Appendix $2.5^{5}$ Further, in an attempt to capture the development of electoral support for the traditionally established parties over time, we calculate the middle vote share (MVS), which is equal to the sum of the vote shares from those political parties that are not categorized as far-left/right, i.e. those parties that do not possess populist and/or radical positions.

Our indicators for average income inequality, the pre-tax and the post-tax Gini coefficients (GiniMarket and GiniNet, respectively) stem from the Standardized World Income Inequality Database (SWIID) by Solt (2016). GiniMarket indicates income inequality before taxes and transfers, i.e. market income inequality; GiniNet indicates income inequality after taxes and transfers, i.e. net income inequality. We use the SWIID database for several reasons. First, the SWIID database covers a larger numbers of countries and years compared to other inequality datasets, for instance the Luxembourg Income Study Solt (2009). Second, the SWIID database maximizes comparability of income inequality across observations giving the opportunity to realize a more appropriate cross-national research. For instance Acemoglu et al. (2015) uses the SWIID to examine the impact of democracy on inequality on a large number of countries. Third, the SWIID provides the distinction between estimates of income inequality pre-tax and post-transfer. Given this differentiation, we are able to explore the effect of net distribution on political polarization. As indicators of tail income inequality, we use the income shares held by the top $10 \%$ and $20 \%$ and the bottom $10 \%$ and $20 \%$ from the World Bank (2019), as well as the $90 / 10$ ratios. The use of these income shares is motivated by the fact that average income inequality provides only a very limited account of the factual distribution of income within an economy, as well as because the sharp increase in income inequality over the last decades has been primarily driven by an overproportional rise of income at the top of the distribution (Piketty 2014b).

We use various macroeconomic variables as controls. First, we include the unemployment rate, defined as the number of unemployed persons as a percentage of the labor force, as Visser et al. (2014) and March and Rommerskirchen (2015) find evidence for the electorate to turn toward far-left parties when the unemployment rate increases. ${ }^{6}$ Regarding the link between the unemployment rate and far-right parties, while some studies have been able to find a positive relationship (Jackman and Volpert, 1996), most empirical studies have reported either no statistical evidence (Swank and Betz

\footnotetext{
${ }^{5}$ We are aware that this party classification may have some limitations, as many political parties have changed their ideologies and positions over time. Moreover, some political parties have disappeared or have joined other political parties. Unfortunately, to the best of our knowledge, there is no existing dataset which would account for these structural shifts in the analyzed countries.

${ }^{6}$ Moreover, Bartolini (2000) shows that the success of communist parties has been historically more marked in countries with socioeconomic problems.
} 
2003; Lubbers and Scheepers, 2002) or a negative relationship (Knigge, 1998 Lubbers and Scheepers 2000). Second, we include the real GDP per capita expressed in 2011 US dollars from the Maddison Project Database, Version 2018 (Bolt et al., 2018), which provides comparable data on income levels for a broad sample of countries, as macroeconomic performance is often found to be related with voting outcomes. According to the so-called clientele hypothesis, far-left parties may benefit in economically hard times (Rattinger, 1981; Nannestad and Paldam, 1994), not least because far-left governments are likely to pursue redistributive policies that benefit lower-income groups by taxing the rich (Kelley and Evans, 1993). Further, we also include the growth rate of the real house price index as provided by the OECD (2017), as a considerable increase in residential property prices could negatively affect traditionally established parties and government coalition parties, and positively affect far-left/right parties. We include housing credit (in real terms), which describes the amount of money that is provided by banks to households as a further control variable, the data for this variable is obtained from the Bank for International Settlements (2017), as well as the inflation rate, measured as the annual growth rate of the GDP implicit deflator from the World Bank (2017) and the growth rate of the government expenditures to GDP ratio. Furthermore, we include two dummy variables. The first dummy variable is a recession dummy constructed by applying the Bry and Boschan (1971) algorithm to the quarterly real GDP per capita series from the Federal Reserve Bank of St. Louis (the list of all recessions in each country as identified by this algorithm can be found in Appendix 2.5). The second dummy variable represents systemic financial crises, defined as situations where the banking sector experiences difficulties; more specifically, financial corporations are unable to fulfill their obligations and many of them default on payments, resulting in a situation followed by significant fiscal costs and output losses (Laeven and Valencia, 2008, 2012). This data is taken from the Macrohistory Database by Jordà et al. (2017). A list of systemic financial crises can be found in Appendix 2.5

Finally, we use the data underlying Dreher (2006) KOF Globalization Index as a measure of globalization. This dataset provides information about changes in the degree of globalization of several countries over time. The Globalization Index is constructed as a weighted measure of economic, political, and social components. First, the economic component measures a country's degree of trade flows: goods, services, and capital. Higher levels of the economic component indicate fewer trade barriers. Second, the social component consists of migration rates and the flow of information related to access to TV and the internet, among others. Finally, the political component indicates the level of international integration in terms of numbers of membership of international treaties. Last but not least, we also include the voter turnout rate from International IDEA (2019) as a control variable, as voters' dissatisfaction may not necessarily lead to an abrupt change in their voting behavior but, instead, may first crystalize in a temporary voting absence. Summary statistics of all variables used throughout this paper are reported in Appendix 2.5

Figure 2.1 illustrates the two versions of the Gini coefficient (pre- and after tax) as reported by Solt (2016) (left graph) and the evolution of the annual cross-country average voting shares for far-left 
(FLVS), middle or mainstream (MVS) and far-right (FRVS) parties.

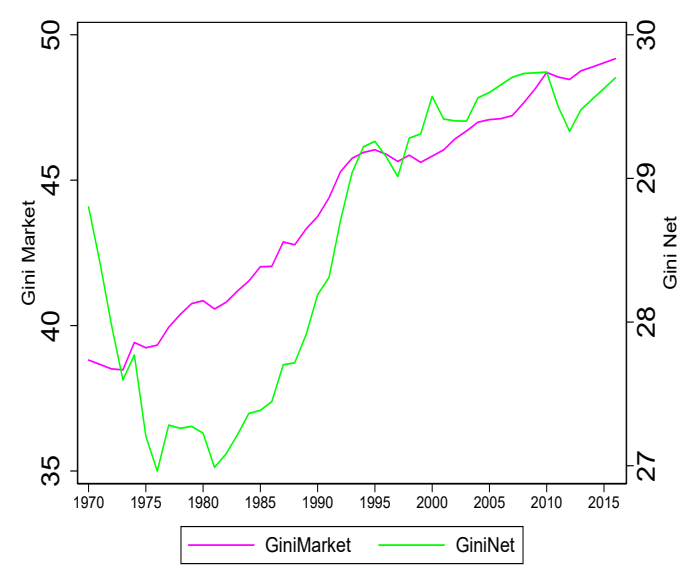

(a) GiniMarket vs GiniNet

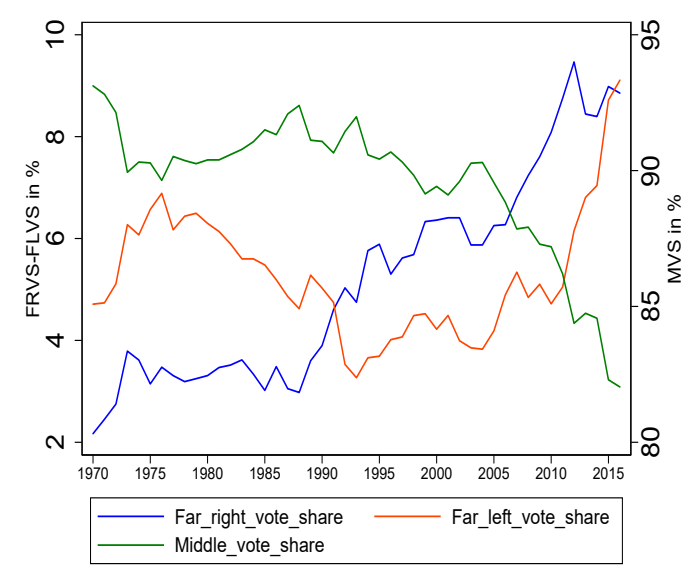

(b) Extreme left- and -right vs middle vote share

Figure 2.1: Income inequality measures and parliamentary vote shares (cross-country averages). Sources: Döring and Manow (2015) and Solt (2016).

Figure 2.1a illustrates the fact that the sharp increase in income inequality over the last two decades (see e.g. Stiglitz, 2013), measured in terms of the pre-tax and after tax Gini coefficient (GiniMarket and GiniNet, respectively) has gone hand in hand with the surge in political extremism (both far-left and far-right) and the decline in electoral support for moderate parties on both the left and the right. This issue leads us to think that inequality may be related to political polarization in a positive and systematic manner.

Figure $2.1 \mathrm{~b}$ indicates that there was little variation between the extreme (far-left or far-right) vote share and the middle vote share through the 1970s and 1980s. However in the 1990s, the extreme vote share started to increase, implying a decrease in the middle vote share. From 1990 to 2016, the extremist parties have more than doubled their presence in national parliaments on average. As can be observed, while far-right parties had a more or less constant vote share in the first two decades of our sample, the far-left parties experienced a steep decline in the parliamentary presence during the 1980s, which can be related to the decline of the political influence of the Soviet Union. Since the beginning of the 1990s, however, both far-left and far-right parties have experienced a surge in electoral support by the population, while moderate left and right parties have experienced a significant decline in their parliamentary representation. ${ }^{7}$

\footnotetext{
${ }^{7}$ It is also important to note that some countries in our sample data illustrate a relatively higher presence of radical and populist parties on both extremes of the political spectrum. The presence of far-left parties is relatively higher in Greece, Spain, Italy, and Ireland compared to the other countries of the sample. Similarly, the presence of far-right parties is more pronounced in Austria, Denmark, Finland, Norway, and Switzerland compared to the other countries of the sample.
} 


\subsubsection{Methodology}

We use panel OLS regressions with fixed effects to investigate how changes in income inequality as well as social changes can influence electoral choices. More specifically, we estimate different specifications with five different dependent variables: the far-left vote share (FLVS), the middle vote share (MVS), and the far-right vote share (FRVS). The general regression model can be described as follows:

$$
\begin{aligned}
& F L V S_{i t}=\beta G_{i t-1}+\delta E_{c o G l o b} i t-1+\gamma \text { SocGlob }_{i t-1}+\zeta \text { PolGlob }_{i t-1}+X_{i t-1} \theta+\alpha_{i}+\kappa_{t}+\epsilon_{i t}, \\
& M V S_{i t}=\beta G_{i t-1}+\delta E_{c o G l o b} i t-1+\gamma \text { SocGlob }_{i t-1}+\zeta \text { PolGlob }_{i t-1}+X_{i t-1} \theta+\alpha_{i}+\kappa_{t}+\epsilon_{i t}, \\
& F R V S_{i t}=\beta G_{i t-1}+\delta E_{c o G l o b} i t-1+\gamma \text { SocGlob }_{i t-1}+\zeta \text { PolGlob }_{i t-1}+X_{i t-1} \theta+\alpha_{i}+\kappa_{t}+\epsilon_{i t},
\end{aligned}
$$

where subscripts $i=1, \ldots, N$ denote the respective countries, and $t=1, \ldots, T$ the time index. $G_{t}$ is a $N \times 1$ vector containing alternative income inequality variables (to be specified below), and $E c o G l o b_{t}, S_{o c G l o b}$ and PolGlob $_{t}$ are $N \times 1$ vectors containing the economic, social and political globalization proxies, respectively, to be specified in detail below. $X_{t}$ is an $N \times K$ matrix and $\theta$ is a $K \times 1$ vector, with $K$ being the number of further explanatory variables to be described in the next subsection. $\alpha_{i}$ is an $N \times 1$ vector of country fixed effects, $\kappa_{t}$ is a $T \times 1$ vector of time effects and finally $\epsilon_{i t}$ is an $N \times 1$ vector of uncorrelated disturbances with zero mean and heteroscedastic country-specific variances $\sigma_{i, \epsilon}^{2}{ }^{8}$ Accordingly, the significance levels reported in all following tables are based on heteroscedasticity-robust standard errors.

At this point it is worth highlighting the potential endogeneity that our main explanatory variables may be subject to which could make a causal interpretation of our estimates problematic. This potential problem could be circumvented by the use of appropriate instruments for our measures of income inequality. This is, however, not a straightforward task. Recently, Krieger and Meierrieks (2019), when investigating the effect of income inequality on terrorism, use the share of mature-aged cohorts (i.e. persons between the ages of 40 and 59) in the country's working age population as an instrument for the Gini coefficient since, as they argue, when mature-aged cohorts are relatively large less income inequality may arise due to more labor market competition. They consider however a much large number of countries (114 in total), so that they have a much larger variation in their sample as we do. Further, this variable is not suitable as an instrument in our analysis as a mature population is likely to systematically vote for traditional parties. Another alternative, which may be considered as an valid instrument for income inequality, its the ten-year-lag. This instrument is also discussed by Krieger and Meierrieks (2019), but the implementation of this instrument will considerably reduced the sample data, especially in our case since we are analyzing election periods. Similarly, it is quite challenging to find any valid instrument for the tail income inequality, i.e. the top $10 \%$ and $20 \%$ income share, as well as the bottom $10 \%$ and $20 \%$ income share.

\footnotetext{
${ }^{8}$ We opt for this option and do use a clustering scheme for the possible cross-country-correlation as most cross-country effects may be reflected in similar macroeconomic developments and not on unexplained disturbances.
} 
In order to circumvent this potential problem, all explanatory variables in our analysis enter the regression models with a lag, where the $t-1$ dating refers not to the previous year, but to the previous election period. Given the asynchronicity of the electoral cycles in our country sample we believe that the potential endogeneity problem in our regressions, while existent, may not be too relevant to invalidate the following results.

\subsubsection{Estimation Results}

\section{Average Income Inequality}

We start by discussing our econometric results using the after-tax Gini coefficient as the economic inequality measure. ${ }^{9}$ We compare two estimation samples: 1970-2016 and 2000-2016. The reason for the analysis of the second sample is twofold: first we hope to gain deeper insights into more recent developments, and second, we would like to investigate whether the introduction of the euro, which affected a large number of countries in our dataset, may represent an important structural shift in many dimensions.

Table 2.1: Panel OLS regressions with GiniNet (all countries). Sample: 1970-2016

\begin{tabular}{lcccccc}
\hline \hline & \multicolumn{2}{c}{ FLVS } & \multicolumn{2}{c}{ MVS } & \multicolumn{2}{c}{ FRVS } \\
& NTE & TE & NTE & TE & NTE & TE \\
\hline GiniNet $(t-1)$ & $0.349^{*}$ & 0.214 & $-0.302^{* *}$ & -0.172 & 0.105 & 0.045 \\
Unemployment rate $(t-1)$ & $0.375^{* *}$ & $0.371^{* *}$ & $-0.278^{* *}$ & $-0.286^{* *}$ & 0.044 & 0.060 \\
RGDP growth $(t-1)$ & $-0.228^{* * *}$ & $-0.230^{* * *}$ & $0.263^{* *}$ & $0.219^{* *}$ & -0.166 & -0.099 \\
Inflation $(t-1)$ & 0.031 & 0.076 & 0.016 & -0.005 & -0.054 & -0.067 \\
Credit HH/GDP growth $(t-1)$ & -0.019 & $-0.272^{* *}$ & 0.036 & 0.129 & -0.035 & 0.076 \\
Real house price growth $(t-1)$ & 0.093 & 0.045 & $-0.116^{* *}$ & -0.052 & 0.081 & 0.033 \\
Gov. exp/GDP growth $(t-1)$ & $-0.187^{* * *}$ & $-0.212^{* * *}$ & $0.160^{* *}$ & $0.137^{*}$ & -0.054 & 0.005 \\
Recession dummy $(t-1)$ & 0.048 & 0.079 & -0.011 & -0.001 & -0.031 & -0.076 \\
Financial crisis dummy $(t-1)$ & 0.041 & 0.042 & -0.036 & -0.023 & 0.013 & -0.007 \\
Economic glob. $(t-1)$ & -0.251 & -0.108 & $0.283^{*}$ & 0.170 & -0.174 & -0.146 \\
Social glob. $(t-1)$ & $-0.552^{*}$ & $-0.857^{* *}$ & 0.097 & $0.333^{*}$ & $0.399^{* * *}$ & $0.349^{* *}$ \\
Political glob. $(t-1)$ & 0.148 & 0.243 & -0.042 & -0.148 & -0.083 & -0.019 \\
\hline Observations & 174 & 174 & 174 & 174 & 174 & 174 \\
\hline \hline
\end{tabular}

Standardized beta coefficients, ${ }^{*} p<0.10,{ }^{* *} p<0.05,{ }^{* * *} p<0.01$ significance levels

\footnotetext{
${ }^{9}$ We also estimated the following regression models using the pre-tax variant of the Gini coefficient (GiniMarket). As this variable is only indirectly related to the actual income distribution perceivable by households in an economy, the results of those regressions were much weaker than the ones using the after-tax Gini coefficient (GiniNet), as expected. These and all other results no present in the Appendices are available upon request.
} 
We estimate the regressions for the far-left (FLVS), the far-right (FRVS) and middle or mainstream voting shares (MVS) using the first lags $(t-1)$ of the explanatory variables to account for a possible endogeneity bias. Further, since the independent variables have different dimensions, we report the standardized regression coefficients to interpret our results in a more intuitive way. ${ }^{10}$

Table 2.1 shows the standardized coefficients of the panel OLS regressions for the FLVS, the FRVS and the MVS without and with time effects (NTE and TE, respectively) included in the regressions using the first lag $(t-1)$ of the explanatory variables and with the GiniNet variable for the estimation sample 1970-2016.

In general terms, the estimation results reported in Table 2.1 are in line with well established knowledge concerning the impact of macroeconomic variables on voting behavior. Independently of whether or not time effects are included in the panel regressions, poor economic performance (represented by an increase in the unemployment rate and by a decrease in the growth rate of the real GDP per capita) increases electoral support for far-left parties (FLVS), and decreases the support for middle or mainstream parties (which are likely to be or have been part of the government and therefore may also be partly responsible for the upswing in economic activity). These two variables are statistically significant at the $5 \%$ level. These results show that far-left parties benefit from economic downturns. The standardized coefficient of the unemployment rate on FLVS 0.37 can be interpreted as follows: an increase in the unemployment rate by one standard deviation increases the FLVS by 0.37 standard deviations on average. Interestingly, far-right party voting is not positively (or negatively) related with changes of the unemployment rate. These findings are consistent with those of Visser et al. (2014) and March and Rommerskirchen (2015), who argue that the electorate shifts toward far-left parties when the unemployment rate increases. Similarly, a higher growth rate of the government-expenditures-to-GDP-ratio can be associated with stronger support for mainstream parties and a weaker electoral support of far-left parties. Further, a higher social globalization seems to be associated with a higher support both of middle and far-right parties in detriment of far-left parties; This result supports to some extent the cultural backlash hypothesis put forward by Inglehart and Norris (2016) to explain the electoral success of Donald Trump. In addition, neither the recession dummy nor the financial crisis dummy seems to be able to explain the electoral support for far-left or far-right parties in contrast to the findings of Funke et al. (2016), who however consider a much larger time span in their study than we do in this paper.

As for the income inequality variable (the GiniNet coefficient), the results are much less robust for the estimated period, as a statistically significant influence of the expected sign (positive for the FLVS and negative for the MVS) is only found in the corresponding panel regressions without time

\footnotetext{
${ }^{10}$ See Bring (1994), who discusses two possibilities of calculating standardized regression coefficients. In this paper, we calculate the standardized regression coefficients by multiplying the estimated coefficient with the ratio between the standard deviation of the independent variable with respect to the standard deviation of the dependent variable: $B_{i}=\hat{\beta}_{i} \cdot\left(\frac{\sigma_{i}}{\sigma_{y}}\right)$.
} 
effects. On a first sight, our data and estimation methodology does not seem to support the notion that economic inequality influences significantly the voting behavior in the analyzed countries. ${ }^{11}$

Table 2.2: Panel OLS regressions with GiniNet (all countries). Subsample: 2000-2016

\begin{tabular}{lcccccc}
\hline \hline & \multicolumn{2}{c}{ FLVS } & \multicolumn{2}{c}{ MVS } & \multicolumn{2}{c}{ FRVS } \\
& NTE & TE & NTE & TE & NTE & TE \\
\hline GiniNet $(t-1)$ & 0.200 & 0.306 & $-0.563^{* * *}$ & $-0.673^{* * *}$ & $0.595^{* *}$ & $0.654^{* *}$ \\
Unemployment rate $(t-1)$ & $0.757^{* * *}$ & $0.803^{* * *}$ & $-0.584^{* * *}$ & $-0.565^{* * *}$ & 0.137 & 0.070 \\
RGDP growth $(t-1)$ & $-0.231^{* * *}$ & $-0.210^{* * *}$ & $0.188^{* *}$ & $0.229^{* * *}$ & -0.055 & -0.129 \\
Inflation $(t-1)$ & 0.085 & 0.178 & -0.090 & -0.104 & 0.048 & -0.013 \\
Credit HH/GDP growth $(t-1)$ & -0.095 & $-0.264^{*}$ & $0.180^{*}$ & 0.297 & -0.164 & -0.176 \\
Real house price growth $(t-1)$ & $0.167^{* *}$ & $0.185^{* * *}$ & $-0.202^{* * *}$ & $-0.235^{* * *}$ & $0.131^{*}$ & 0.160 \\
Gov. exp/GDP growth $(t-1)$ & $-0.188^{* * *}$ & $-0.146^{*}$ & 0.033 & -0.065 & 0.119 & $0.217^{*}$ \\
Recession dummy $(t-1)$ & -0.006 & 0.048 & 0.037 & 0.018 & -0.044 & -0.067 \\
Financial crisis dummy $(t-1)$ & $0.076^{*}$ & 0.092 & -0.053 & -0.054 & 0.006 & -0.006 \\
Economic glob. $(t-1)$ & 0.032 & -0.051 & -0.082 & -0.174 & 0.084 & 0.283 \\
Social glob. $(t-1)$ & 0.050 & 0.092 & -0.142 & -0.179 & 0.151 & 0.165 \\
Political glob. $(t-1)$ & 0.009 & -0.040 & -0.217 & -0.299 & $0.289^{*}$ & $0.444^{*}$ \\
\hline Observations & 87 & 87 & 87 & 87 & 87 & 87 \\
\hline \hline
\end{tabular}

Standardized beta coefficients, ${ }^{*} p<0.10,{ }^{* *} p<0.05,{ }^{* * *} p<0.01$ significance levels

Table 2.2 reports the estimation results for the 2000-2016 subsample. Three interesting differences are worth discussing: First and foremost, the income inequality variable did not seem to have a robust impact on any of the endogenous variables in the 1970-2016 sample, in the 2000-2016 subsample its coefficient is highly statistically significant and of a important magnitude in the MVS and the FRVS regressions. Accordingly, economic inequality can be associated with a higher support for far-right parties to the detriment of established mainstream parties. This finding corroborates the ideas of Jesuit et al. (2009), who suggested that higher levels of income inequality increase electoral support for far-right parties. As the sharp increase in economic inequality cannot be considered an exogenous process, but it has been instead promoted by active tax and labor market policies, the rise of far-right parties can be considered, to a certain extent, a product of political decisions. Further, while the

\footnotetext{
${ }^{11}$ To test the robustness of our results, we also ran our regressions using the average of all independent variables in the periods between the parliamentary elections for each country. For example, parliamentary elections in Italy took place in $(\ldots), 1996,2001,2006,(\ldots)$. Then, for the observation of the year 2001 in Italy, we calculate the average from 1996-2000. For the observations of the year 2006, we calculate the average from 2001 to 2005 , and so on. This calculation was done for all independent variables in all countries. Since it was not possible to make a meaningful calculation of the year dummy variables in these regressions, we exclude them from these regression variants, i.e. we exclude the recession and financial crises dummies. The results of the panel OLS regressions with fixed effects with the averages of the independent variables using the GiniNet variable are quite similar to the ones reported in Tables 2.1 and 2.2 and are available upon request.
} 
standardized coefficients of the real GDP per capita estimated in this recent subsample are quite similar to those reported in Table 2.1, the standardized coefficients of the unemployment rate in the FLVS and the MVS regressions are about twice as large as in the previous estimation, suggesting that the state of the labor market may have gained relevance for particularly for the support of farleft parties. Last but not not least, the growth rate of house prices in real terms has also gained in relevance for the support of far-left parties, as the positive and statistically significant coefficients reported in Table 2.2 indicate. This is also related to a clientele hypothesis after which far-left parties may benefit from higher housing prices since their ideological position supports market regulation and government intervention. Finally, it is worth highlighting the fact that besides from the social component of the Globalization Index, electoral support for far-right parties seems to be decoupled from macroeconomic fundamentals when the complete estimation sample is considered. ${ }^{12}$

In order to investigate the apparent structural shift in the relationships among income inequality, globalization, and electoral support for far-left and far-right parties in more detail, we estimate rolling regressions using an estimation window of the length of the first subsample (1970-1999) up to the last estimation subsample (1987-2016) resulting in 18 periods in total. This procedure may serve as an important opportunity to advance the understanding of the political consequences of a major economic integration via the introduction of the Euro, as previously mentioned. ${ }^{13}$

Figure 2.2 shows the point estimates and the corresponding standard errors of four key coefficients: the GiniNet on the FLVS (Figure 2.2a), the GiniNet on the FRVS (Figure 2.2c), the social component of the Globalization Index on the FLVS (Figure 2.2d), on the MVS (Figure 2.2d) and on the FRVS (Figure 2.2f) from the rolling panel regressions with different subsamples with a fixed estimation window length from 1970-1999 up to 2000-2016. While the point estimates in Figures 2.2a are relatively constant over time, the GiniNet seems to exert an increasingly negative positive effect on the MVS (FRVS) in recent times, corroborating the estimation results summarized in Table 2.3 Income inequality thus seems to have become a major driving force behind the rise of far-right parties in detriment of mainstream parties in recent times. Interestingly, Figures $2.2 \mathrm{~d} \cdot 2.2 \mathrm{f}$ which would correspond to the cultural backlash hypothesis of Inglehart and Norris (2016) for the United States, do not seem to be corroborated by data stemming from other advanced economies.

\footnotetext{
${ }^{12}$ Additionally, we estimated the regression models excluding those parliamentary elections where far-left/right parties were part of the government coalition. This could be an important determinant, as Dornbusch and Edwards (1989) pointed out the negative consequences of having populist governments. The results are robust in the sense that economic distress plays an important role for the FLVS, but not for the FRVS. Again, the GiniNet coefficient loses its statistical significance when the whole sample is analyzed, but remains statistically significant when recent years. These estimation results are also available upon request.

${ }^{13}$ It should be noted, however, that this procedure comes at the cost of estimation accuracy because of the shorter estimation sample used in each of the rolling regressions.
} 


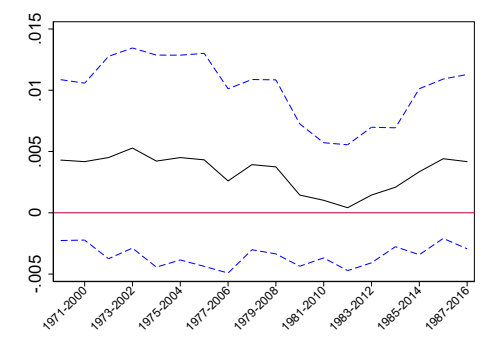

(a) GiniNet Coefficient on FLVS

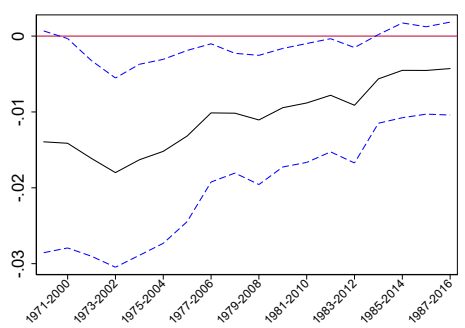

(d) SocGlob Coefficient on FLVS

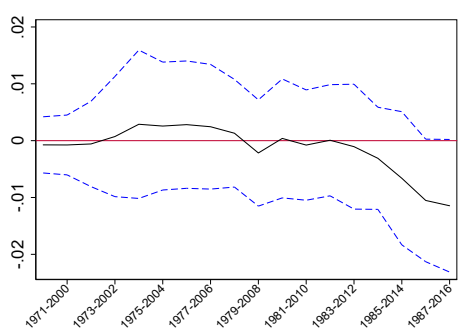

(b) GiniNet Coefficient on MVS

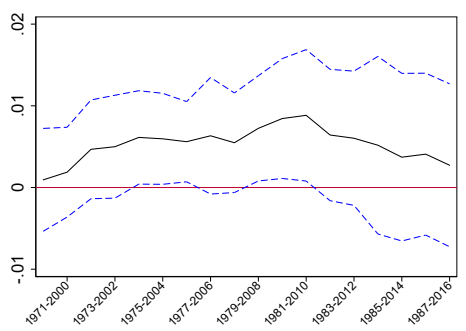

(e) SocGlob Coefficient on MVS

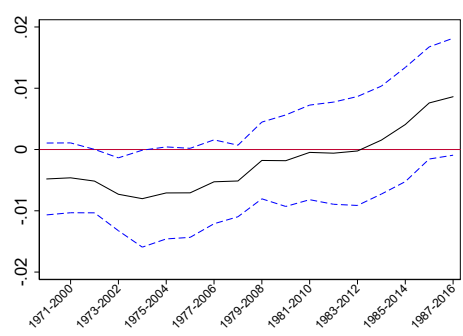

(c) GiniNet Coefficient on FRVS

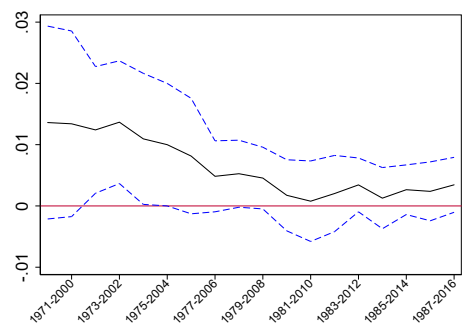

(f) SocGlob Coefficient on FRVS

Figure 2.2: Time-varying coefficients (not standardized) obtained from rolling panel regressions of FLVS, MVS and FRVS.

Table 2.3: Panel OLS regressions with GiniNet (European countries). Sample: 1970-2016

\begin{tabular}{lcccccc}
\hline \hline & \multicolumn{2}{c}{ FLVS } & \multicolumn{2}{c}{ MVS } & \multicolumn{2}{c}{ FRVS } \\
& NTE & TE & NTE & TE & NTE & TE \\
\hline GiniNet $(t-1)$ & $0.451^{* *}$ & $0.725^{* * *}$ & $-0.387^{* *}$ & $-0.515^{*}$ & 0.095 & 0.001 \\
Unemployment rate $(t-1)$ & $0.407^{* *}$ & $0.401^{* *}$ & $-0.330^{* *}$ & -0.324 & 0.058 & 0.057 \\
RGDP growth $(t-1)$ & $-0.278^{* * *}$ & $-0.167^{* *}$ & $0.358^{* * *}$ & $0.279^{* * *}$ & $-0.228^{*}$ & $-0.227^{*}$ \\
Inflation $(t-1)$ & 0.096 & $0.416^{* * *}$ & -0.016 & -0.141 & -0.074 & -0.217 \\
Credit HH/GDP growth $(t-1)$ & -0.053 & $-0.350^{* *}$ & 0.065 & 0.215 & -0.039 & 0.047 \\
Real house price growth $(t-1)$ & 0.111 & 0.023 & $-0.160^{* * *}$ & -0.081 & 0.116 & 0.092 \\
Gov. exp/GDP growth $(t-1)$ & $-0.170^{* * *}$ & $-0.286^{* * *}$ & $0.169^{* *}$ & $0.188^{*}$ & -0.068 & 0.022 \\
Recession dummy $(t-1)$ & -0.009 & 0.001 & 0.031 & 0.033 & -0.036 & -0.047 \\
Financial crisis dummy $(t-1)$ & 0.056 & 0.017 & -0.053 & 0.057 & 0.019 & -0.098 \\
Economic glob. $(t-1)$ & $-0.360^{*}$ & $-0.607^{*}$ & $0.312^{*}$ & $0.457^{* *}$ & -0.079 & -0.038 \\
Social glob. $(t-1)$ & -0.404 & -0.060 & 0.067 & 0.067 & $0.310^{*}$ & -0.034 \\
Political glob. $(t-1)$ & 0.211 & $0.515^{* * *}$ & -0.096 & $-0.324^{* * *}$ & -0.076 & -0.058 \\
\hline Observations & 131 & 131 & 131 & 131 & 131 & 131 \\
\hline \hline
\end{tabular}

Standardized beta coefficients, ${ }^{*} p<0.10,{ }^{* *} p<0.05,{ }^{* * *} p<0.01$ significance levels 
Table 2.4: Panel OLS regressions with Gini Net (European countries). Subsample: 2000-2016

\begin{tabular}{lcccccc}
\hline \hline & \multicolumn{2}{c}{ FLVS } & \multicolumn{2}{c}{ MVS } & \multicolumn{2}{c}{ FRVS } \\
& NTE & TE & NTE & TE & NTE & TE \\
\hline GiniNet $(t-1)$ & 0.265 & $0.534^{* *}$ & $-0.688^{* * *}$ & $-0.880^{* * *}$ & $0.642^{*}$ & $0.644^{*}$ \\
Unemployment rate $(t-1)$ & $0.889^{* *}$ & $1.012^{* * *}$ & $-0.743^{* *}$ & $-0.901^{* * *}$ & 0.148 & 0.240 \\
RGDP growth $(t-1)$ & $-0.268^{* * *}$ & $-0.154^{* *}$ & $0.284^{* * *}$ & $0.264^{* * *}$ & -0.121 & -0.199 \\
Inflation $(t-1)$ & 0.128 & 0.270 & -0.118 & -0.197 & 0.036 & 0.008 \\
Credit HH/GDP growth $(t-1)$ & -0.025 & -0.030 & 0.185 & 0.120 & $-0.214^{*}$ & -0.126 \\
Real house price growth $(t-1)$ & $0.230^{* * *}$ & $0.226^{* *}$ & $-0.288^{* * *}$ & $-0.349^{* * *}$ & $0.162^{*}$ & 0.242 \\
Gov. exp/GDP growth $(t-1)$ & $-0.177^{* *}$ & $-0.224^{*}$ & 0.034 & -0.048 & 0.117 & 0.264 \\
Recession dummy $(t-1)$ & -0.051 & -0.030 & 0.083 & 0.055 & -0.060 & -0.043 \\
Financial crisis dummy $(t-1)$ & $0.071^{*}$ & 0.037 & -0.073 & 0.068 & 0.029 & -0.120 \\
Economic glob. $(t-1)$ & -0.048 & 0.069 & -0.016 & -0.260 & 0.064 & 0.270 \\
Social glob. $(t-1)$ & $0.149^{* *}$ & 0.267 & -0.133 & -0.274 & 0.036 & 0.110 \\
Political glob. $(t-1)$ & 0.053 & -0.055 & -0.250 & -0.154 & $0.272^{*}$ & 0.246 \\
\hline Observations & 69 & 69 & 69 & 69 & 69 & 69 \\
\hline \hline
\end{tabular}

Standardized beta coefficients, ${ }^{*} p<0.10,{ }^{* *} p<0.05, * * * p<0.01$ significance levels

Tables 2.3 and 2.4 report the estimation results obtained using European countries only, i.e., not taking into account Australia, Canada, Japan and the United States. In general terms, the results reported in these two tables are quite in line with those obtained using the full set of countries with a single but key exception: When the full estimation sample 1970-2016 is considered the estimated inequality coefficient in the FLVS regression is highly statistically significant independently of whether time fixed effects are included in the regression or not. In this context, the statistically significant and positive coefficient for the FRVS in the 2000-2016 European sample is even more remarkable, as it indicates a radical shift in the way how income inequality may affect electoral outcomes, away from supporting far-left parties towards benefiting far-right parties.

\section{Tail Income Inequality}

A main shortcoming of the Gini coefficient (either pre- or after tax) is the well known fact that it underestimates changes at the tails of the income distribution (Atkinson 1970).

Table 2.5 reports the results using the 90/10 income share ratio as an aggregate measure of tail income inequality. As in the previous cases, here we also find the robust results concerning the macroeconomic performance variables (unemployment rate and real GDP per capita growth), as well as a statistically significant and positive (negative) influence on the FLVS (MVS) for the 1970-2016, and no significant effect on the FRVS. When the 2000-2016 subsample is considered, the previously found 
Table 2.5: Panel OLS regressions with income share ratio 90_10 (all countries). Sample: 1970-2016

\begin{tabular}{lcccccc}
\hline \hline & \multicolumn{2}{c}{ FLVS } & \multicolumn{2}{c}{ MVS } & \multicolumn{2}{c}{ FRVS } \\
& NTE & TE & NTE & TE & NTE & TE \\
\hline Ratio 90_10 $(t-1)$ & $0.255^{*}$ & $0.256^{* *}$ & $-0.291^{* *}$ & $-0.236^{*}$ & $0.192^{* *}$ & 0.114 \\
Unemployment rate $(t-1)$ & $0.425^{* *}$ & $0.798^{* * *}$ & $-0.287^{*}$ & $-0.569^{* * *}$ & 0.040 & 0.119 \\
RGDP growth $(t-1)$ & $-0.187^{* * *}$ & $-0.217^{* * *}$ & $0.210^{* * *}$ & $0.253^{* * *}$ & -0.136 & -0.172 \\
Inflation $(t-1)$ & -0.060 & $0.152^{*}$ & 0.052 & -0.071 & -0.021 & -0.030 \\
Credit HH/GDP growth $(t-1)$ & -0.081 & -0.035 & 0.056 & 0.096 & -0.010 & -0.107 \\
Real house price growth $(t-1)$ & 0.068 & $0.175^{* * *}$ & -0.074 & $-0.210^{*}$ & 0.046 & 0.147 \\
Gov. exp/GDP growth $(t-1)$ & -0.143 & $-0.248^{* *}$ & 0.075 & 0.103 & 0.017 & 0.068 \\
Recession dummy $(t-1)$ & 0.006 & -0.012 & 0.068 & 0.101 & -0.102 & -0.132 \\
Financial crisis dummy $(t-1)$ & 0.045 & 0.020 & -0.051 & 0.049 & 0.033 & -0.086 \\
Economic glob. $(t-1)$ & -0.248 & -0.069 & 0.256 & 0.107 & -0.149 & -0.092 \\
Social glob. $(t-1)$ & -0.018 & 0.145 & -0.107 & -0.075 & $0.168^{*}$ & -0.018 \\
Political glob. $(t-1)$ & 0.010 & -0.272 & 0.026 & 0.075 & -0.045 & 0.128 \\
\hline Observations & 108 & 108 & 108 & 108 & 108 & 108 \\
\hline \hline
\end{tabular}

Standardized beta coefficients, ${ }^{*} p<0.10,{ }^{* *} p<0.05, * * * p<0.01$ significance levels

positive effect on FRVS is however not further present, as reported on Table 2.10 in the Appendix $2.5^{14}$

It is however quite likely that changes at the different tails of the income distribution may also have different effects on electoral outcomes because they affect different parts of the population. In order to investigate this issue, we ran separate regressions including the bottom and top $10 \%$ and $20 \%$ income shares. Tables 2.6 and 2.7 report the estimation results for FLVS, MVS and FRVS using the bottom 10\% income share for the estimation samples 1970-2016 and 2000-2016, respectively, without and with time effects.

For the 1970-2016 sample we find a significant and negative (positive) influence of the bottom 10\% income share on the FLVS (MVS) in the panel estimations with time effects. Accordingly, an increase of the bottom $10 \%$ income share by one standard deviation can be associated with a 0.308 standard deviations lower far-left voting share (FLVS), and a 0.344 standard deviations higher middle voting share (MVS). An improvement of the economic conditions of the poorest share of the population seems to be linked with a decrease in electoral support to far-left parties, and a higher approval of mainstream parties when the whole 1970-2016 sample is considered.

As it was the case with the measure of post-tax average income inequality, the estimation results for the 2000-2016 for the bottom 10\% indicate a dramatic shift in the link between income inequality and voting behavior: While the link between this variable and the FRVS was not robust in the full

\footnotetext{
${ }^{14}$ Tables 2.11 and 2.12 report the estimation results with a 80/20 income share ratios for the full and the 2000-2016 sample.
} 
Table 2.6: Panel OLS regressions with bottom 10\% income share (all countries). Sample: 1970-2016

\begin{tabular}{lcccccc}
\hline \hline & \multicolumn{2}{c}{ FLVS } & \multicolumn{2}{c}{ MVS } & \multicolumn{2}{c}{ FRVS } \\
& NTE & TE & NTE & TE & NTE & TE \\
\hline Inc. share bottom $10(t-1)$ & -0.201 & $-0.308^{* *}$ & 0.279 & $0.344^{* *}$ & $-0.223^{*}$ & -0.223 \\
Unemployment rate $(t-1)$ & $0.428^{* *}$ & $0.814^{* * *}$ & -0.281 & $-0.582^{* * *}$ & 0.030 & 0.123 \\
RGDP growth $(t-1)$ & $-0.187^{* * *}$ & $-0.212^{* * *}$ & $0.208^{* *}$ & $0.246^{* * *}$ & -0.133 & -0.166 \\
Inflation $(t-1)$ & -0.039 & $0.187^{*}$ & 0.035 & -0.114 & -0.016 & 0.000 \\
Credit HH/GDP growth $(t-1)$ & -0.142 & -0.093 & 0.121 & 0.134 & -0.049 & -0.110 \\
Real house price growth $(t-1)$ & 0.090 & $0.193^{* * *}$ & -0.098 & $-0.225^{* *}$ & 0.061 & 0.152 \\
Gov. exp/GDP growth $(t-1)$ & -0.110 & $-0.209^{* *}$ & 0.037 & 0.081 & 0.042 & 0.065 \\
Recession dummy $(t-1)$ & 0.001 & -0.004 & 0.071 & 0.091 & -0.101 & -0.126 \\
Financial crisis dummy $(t-1)$ & 0.043 & 0.055 & -0.048 & 0.005 & 0.031 & -0.054 \\
Economic glob. $(t-1)$ & -0.217 & -0.055 & 0.225 & 0.101 & -0.131 & -0.096 \\
Social glob. $(t-1)$ & -0.024 & 0.090 & -0.103 & -0.027 & 0.167 & -0.039 \\
Political glob. $(t-1)$ & -0.032 & $-0.400^{*}$ & 0.094 & 0.241 & -0.105 & 0.003 \\
\hline Observations & 108 & 108 & 108 & 108 & 108 & 108 \\
\hline \hline
\end{tabular}

Standardized beta coefficients, ${ }^{*} p<0.10,{ }^{* *} p<0.05,{ }^{* * *} p<0.01$ significance levels

Table 2.7: Panel OLS regressions bottom 10\% income share (all countries). Subsample: 2000-2016

\begin{tabular}{lcccccc}
\hline \hline & \multicolumn{2}{c}{ FLVS } & \multicolumn{2}{c}{ MVS } & \multicolumn{2}{c}{ FRVS } \\
& NTE & TE & NTE & TE & NTE & TE \\
\hline Inc. share bottom $10(t-1)$ & -0.212 & -0.260 & $0.349^{* * *}$ & $0.457^{* *}$ & $-0.289^{* *}$ & $-0.394^{*}$ \\
Unemployment rate $(t-1)$ & $0.701^{* *}$ & $0.824^{* * *}$ & $-0.538^{* * *}$ & $-0.630^{* * *}$ & 0.113 & 0.130 \\
RGDP growth $(t-1)$ & $-0.213^{* * *}$ & $-0.190^{* * *}$ & $0.219^{* * *}$ & $0.248^{* * *}$ & -0.110 & $-0.171^{*}$ \\
Inflation $(t-1)$ & -0.008 & 0.115 & 0.029 & -0.058 & -0.032 & -0.023 \\
Credit HH/GDP growth $(t-1)$ & 0.036 & -0.059 & 0.011 & 0.100 & -0.047 & -0.084 \\
Real house price growth $(t-1)$ & 0.115 & $0.175^{* *}$ & -0.122 & $-0.231^{* *}$ & 0.064 & 0.161 \\
Gov. exp/GDP growth $(t-1)$ & $-0.189^{* *}$ & $-0.206^{* *}$ & 0.117 & 0.097 & 0.008 & 0.049 \\
Recession dummy $(t-1)$ & -0.010 & -0.005 & 0.070 & 0.086 & -0.087 & -0.113 \\
Financial crisis dummy $(t-1)$ & $0.087^{* *}$ & 0.031 & $-0.110^{* * *}$ & 0.027 & 0.074 & -0.063 \\
Economic glob. $(t-1)$ & -0.140 & 0.037 & 0.090 & -0.152 & 0.001 & 0.175 \\
Social glob. $(t-1)$ & 0.073 & 0.070 & 0.002 & 0.012 & -0.067 & -0.078 \\
Political glob. $(t-1)$ & -0.055 & -0.056 & -0.018 & -0.043 & 0.074 & 0.108 \\
\hline Observations & 80 & 80 & 80 & 80 & 80 & 80 \\
\hline \hline
\end{tabular}

Standardized beta coefficients, ${ }^{*} p<0.10,{ }^{* *} p<0.05, * * * p<0.01$ significance levels 
sample estimation, the bottom $10 \%$ income share appears to be linked in a statistically significant manner not with the FLVS as it was the case when the 1970-2016 sample was considered, but with the FRVS in the 2000-2016 sample, as it was the case with the GiniNet coefficient (see Table 2.2).

Table 2.8: Panel OLS regressions bottom 10\% income share (European countries). Sample: 1970-2016

\begin{tabular}{lcccccc}
\hline \hline & \multicolumn{2}{c}{ FLVS } & \multicolumn{2}{c}{ MVS } & \multicolumn{2}{c}{ FRVS } \\
& NTE & TE & NTE & TE & NTE & TE \\
\hline Inc. share bottom $10(t-1)$ & -0.121 & -0.333 & 0.260 & $0.891^{*}$ & -0.235 & $-0.873^{*}$ \\
Unemployment rate $(t-1)$ & $0.678^{* *}$ & $0.936^{* * *}$ & $-0.505^{*}$ & $-0.737^{* *}$ & 0.068 & 0.147 \\
RGDP growth $(t-1)$ & $-0.195^{* * *}$ & $-0.175^{* * *}$ & $0.261^{* * *}$ & $0.245^{* *}$ & -0.172 & -0.168 \\
Inflation $(t-1)$ & 0.098 & 0.136 & -0.061 & -0.126 & -0.006 & 0.046 \\
Credit HH/GDP growth $(t-1)$ & -0.131 & -0.010 & 0.139 & 0.062 & -0.068 & -0.072 \\
Real house price growth $(t-1)$ & $0.163^{*}$ & $0.198^{* *}$ & $-0.193^{* *}$ & $-0.262^{* *}$ & $0.110^{*}$ & 0.169 \\
Gov. exp/GDP growth $(t-1)$ & -0.040 & $-0.281^{*}$ & -0.027 & 0.049 & 0.070 & 0.180 \\
Recession dummy $(t-1)$ & -0.040 & -0.037 & 0.113 & 0.030 & -0.112 & -0.006 \\
Financial crisis dummy $(t-1)$ & 0.055 & 0.009 & -0.079 & -0.000 & 0.055 & -0.008 \\
Economic glob. $(t-1)$ & $-0.547^{* *}$ & -0.044 & $0.454^{* *}$ & -0.454 & -0.116 & 0.632 \\
Social glob. $(t-1)$ & 0.218 & 0.117 & $-0.299^{* *}$ & -0.043 & $0.201^{* *}$ & -0.047 \\
Political glob. $(t-1)$ & 0.051 & -0.249 & 0.070 & 0.127 & -0.136 & 0.051 \\
\hline Observations & 79 & 79 & 79 & 79 & 79 & 79 \\
\hline \hline
\end{tabular}

Standardized beta coefficients, ${ }^{*} p<0.10,{ }^{* *} p<0.05,{ }^{* * *} p<0.01$ significance levels

Table 2.9: Panel OLS regressions bottom 10\% income share (European countries). Sample: 2000-2016

\begin{tabular}{lcccccc}
\hline \hline & \multicolumn{2}{c}{ FLVS } & \multicolumn{2}{c}{ MVS } & \multicolumn{2}{c}{ FRVS } \\
& NTE & TE & NTE & TE & NTE & TE \\
\hline Inc. share bottom $10(t-1)$ & -0.193 & -0.244 & $0.411^{* *}$ & $0.693^{* *}$ & $-0.350^{*}$ & $-0.665^{*}$ \\
Unemployment rate $(t-1)$ & $0.825^{* *}$ & $0.900^{* * *}$ & $-0.655^{* *}$ & $-0.755^{* * *}$ & 0.088 & 0.148 \\
RGDP growth $(t-1)$ & $-0.257^{* * *}$ & $-0.170^{* * *}$ & $0.277^{* * *}$ & $0.254^{* * *}$ & -0.121 & -0.171 \\
Inflation $(t-1)$ & 0.018 & 0.109 & 0.040 & -0.108 & -0.068 & 0.039 \\
Credit HH/GDP growth $(t-1)$ & 0.074 & -0.010 & -0.011 & 0.064 & -0.053 & -0.073 \\
Real house price growth $(t-1)$ & $0.181^{*}$ & $0.193^{* *}$ & $-0.191^{*}$ & $-0.271^{* *}$ & 0.080 & 0.171 \\
Gov. exp/GDP growth $(t-1)$ & $-0.198^{* *}$ & $-0.268^{*}$ & 0.132 & 0.050 & 0.011 & 0.179 \\
Recession dummy $(t-1)$ & -0.030 & -0.035 & 0.074 & 0.030 & -0.068 & -0.006 \\
Financial crisis dummy $(t-1)$ & $0.100^{* *}$ & 0.009 & $-0.139^{* * *}$ & -0.000 & 0.086 & -0.008 \\
Economic glob. $(t-1)$ & -0.189 & -0.030 & 0.155 & -0.329 & -0.027 & 0.448 \\
Social glob. $(t-1)$ & 0.102 & 0.066 & -0.006 & -0.026 & -0.085 & -0.028 \\
Political glob. $(t-1)$ & -0.030 & -0.058 & -0.035 & 0.032 & 0.072 & 0.013 \\
\hline Observations & 67 & 67 & 67 & 67 & 67 & 67 \\
\hline \hline
\end{tabular}

Standardized beta coefficients, ${ }^{*} p<0.10,{ }^{* *} p<0.05,{ }^{* * *} p<0.01$ significance levels 
When only European countries are considered (see Tables 2.8 and 2.9), we find that the $10 \%$ lowest income share seems to have a stronger and statistically significant positive impact on the FRVS in recent times (2000-2016) as it was also the case with the GiniNet coefficient. ${ }^{15}$

To corroborate these findings we ran rolling panel regressions analogously to those illustrated in Figure 2.2. As it can be clearly observed in the first row of Figure 2.3 these rolling regressions illustrate in a quite clear manner the increasingly stronger link between the bottom $10 \%$ income share and the electoral support for far-right parties in detriment of mainstream parties. Indeed, what appears to be a structural change in the link between the bottom $10 \%$ income share and the electoral success of mainstream and far-right parties seems to have occurred - even in a more pronounced manner than in the case of the GiniNet - over the last 20 years.

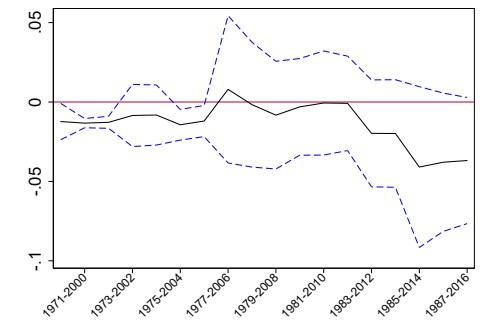

(a) Low $10 \%$ on FLVS

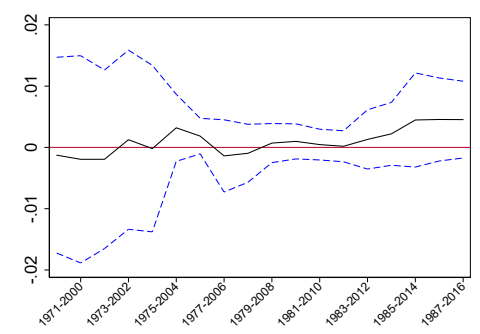

(d) Top $10 \%$ on FLVS

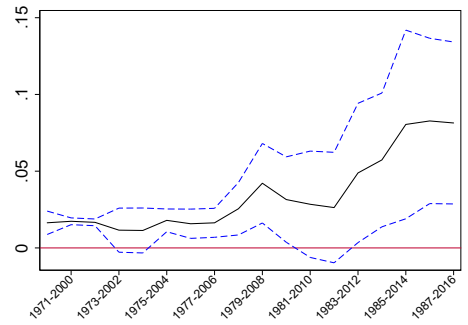

(b) Low $10 \%$ on MVS

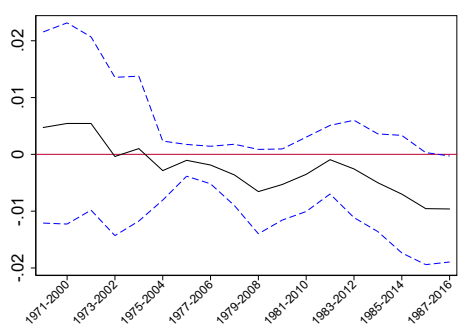

(e) Top $10 \%$ on MVS

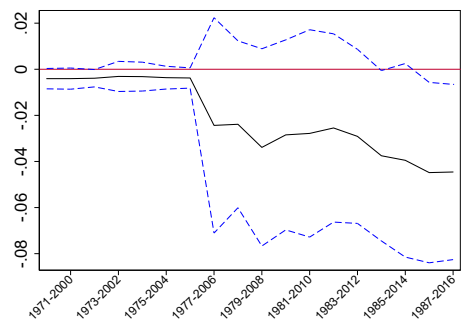

(c) Low $10 \%$ on FRVS

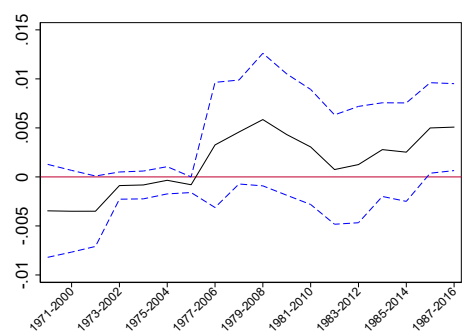

(f) Top $10 \%$ on FRVS

Figure 2.3: Time-varying coefficients obtained from rolling panel regressions of FRVS and FLVS.

We ran similar panel regressions using the top $10 \%$ income share for both estimation samples (Tables 2.17 to 2.24 in the Appendix 2.5), and found no robust statistically significant linkages between this variable and the electoral support for far-left or far-right parties, irrespectively of whether we use all countries in our sample, or only the European ones. In the second row of Figure 2.3 we illustrate the rolling regression estimates of the top $10 \%$ income share coefficients in the FLVS, MVS and FRVS regressions. In contrast to the coefficients corresponding to the bottom $10 \%$ income share depicted

\footnotetext{
${ }^{15}$ Additionally, we ran all these estimations using the bottom $20 \%$ income share as a right-hand side variable finding similar, but less robust results. These estimations can be found in Tables 2.13 through 2.16 in Appendix 2.5
} 
in the panels (b) and (c) in the first row, the empirical evidence show in the panels (e) and (f) for a structural change concerning the link between the top $10 \%$ income share and MVS and FRVS seems much less robust. The link between income inequality and the voting share of far-right parties seems thus to be a story of a higher income inequality driven by the relative deterioration of the economic conditions of the poorer share of the population in the analyzed countries, rather than a story of the Top X percent.

\subsection{Concluding Remarks}

In recent decades, many mature liberal democracies in Europe and beyond have experienced growing social inequality on the one hand, and increasing social protest and political polarization on the other. Yet, despite a great deal of sophisticated quantitative and qualitative research, the link between social and economic change and its political repercussions still requires further work. While it is a well-established finding in scholarship on "economic voting" that governments are penalized for poor economic performance, less is known on the conditions for voters on aggregate to switch to politically radical parties - on the left or right - rather than abstaining or voting for a mainstream opposition party. Not least, scholarship seeking to explain the rise of populist parties on the radical right or left has not specified the conditions for voters to switch to either a radical right-wing or, alternatively, a radical left-wing party. Finally, crises often have a delayed impact, even though most empirical studies on the effect of economic crises have been relatively short-term. Also, the current literature in political science offers few insights into the question whether, and to what extent, the effect of social inequality and other variables has historically changed over time.

This paper seeks to address some of these questions. The answers are normatively important, as they help us to understand the conditions under which social and political grievances are translated into political responses at the systemic level. The responsiveness of liberal democracies is often seen as a key condition for their "input-oriented legitimacy" (Scharpf, 1999). Given the close link between liberal democracies and liberal market economies as institutions, the way discontent with growing inequality is manifested in representative democracies is a crucial question for both sets of institutions. When grievances are manifested by strengthening radical parties of the left and/or right, they will lead to the polarization of political conflict in legislatures and other decision-making bodies, potentially reducing the effectiveness of the system in responding to crises. This constitutes a challenge to the "output-oriented legitimacy" (Scharpf, 1999) of political systems.

It is against this background that we investigate the determinants of political polarization from the demand-side of voters, using a panel analysis based on annual aggregate data for 20 advanced economies between 1970 and 2016. The study covers 291 parliamentary elections. Our empirical analyses deliver a variety of interesting insights to complement the existing literature on economic 
voting: On the one hand, our analyses support the accepted wisdom of the literature on economic voting, showing that government parties get penalized for poor economic performance. In addition, our results support accounts claiming that far-left parties have benefited from periods of weak economic performance, while mainstream parties (irrespective of their government status) have been penalized at the polls. Moving beyond standard accounts, we find that income inequality has become a main driver for the growing electoral support of far-right parties, while it is simultaneously associated with electoral losses for mainstream parties. Rather than focusing on a "second", cultural dimension of political conflict (a claim investigated in a large number of analyses), we demonstrate that hard breadand-butter issues and social inequality matter in explaining the recent popularity of far-right parties in Western European democracies. As previously mentioned, the link between income inequality and far-right parties seems to be a story of a higher income inequality driven by the relative deterioration of the economic conditions of the poorer share of the population in the analyzed countries, than a story of the Top X percent.

Our findings on the effect of globalization and of social change suggest that potential cultural factors are an important part of the story, but that social inequality should not be ignored and is an important part of a more complete explanation. Our rolling panel design also reveals that the effects of many of the explanatory variables have changed over time. Not only do our findings suggest a time-varying and/or regime-dependent nature of voter support for far-left and far-right parties, but also an interesting interplay between macroeconomic and social electoral outcomes. In particular, both average and tail income inequality (the former measured by the post-tax Gini coefficient and the latter by the bottom $10 \%$ income share) seem to have played a favouring role in the increasing electoral success of far-right parties in recent times. While we do not explicitly model the effect of different government policies (e.g. redistributive taxation or welfare expenditure), policy studies demonstrate that the increase in income inequality is not a natural phenomenon, but the result of policy choices made by governments, including policies on taxation benefiting more affluent groups in Western societies to the detriment of groups at the lower end of the income ladder (OECD 2010). Thus, the rise of far-right parties can be considered a home-made problem to a certain extent that can be addressed through public policy. 


\subsection{Appendix to Chapter 2}




\section{Parliamentary Elections 1970-2016}

\begin{tabular}{|c|c|}
\hline Australia & $\begin{array}{l}\text { 1969, 1972, 1974, 1975, 1977, 1980, 1983, 1984, 1987, 1990, 1993, 1996, 1998, } \\
2001,2004,2007,2010,2013,2016\end{array}$ \\
\hline Austria & $\begin{array}{l}1970,1971,1975,1979,1983,1986,1990,1994,1995,1999,2002,2006,2008, \\
2013\end{array}$ \\
\hline Belgium & $\begin{array}{l}\text { 1968, 1971, 1974, 1977, 1978, 1981, 1985, 1987, 1991, 1995, 1999, 2003, 2007, } \\
\text { 2010, } 2014\end{array}$ \\
\hline Canada & $\begin{array}{l}\text { 1968, 1972, 1974, 1979, 1980, 1984, 1988, 1993, 1997, 2000, 2004, 2006, 2008, } \\
2011,2015\end{array}$ \\
\hline Switzerland & $1967,1971,1975,1979,1983,1987,1991,1995,1999,2003,2007,2011,2015$ \\
\hline Germany & $1969,1972,1976,1980,1983,1987,1990,1994,1998,2002,2005,2009,2013$ \\
\hline Denmark & $\begin{array}{l}\text { 1968, 1971, 1973, 1975, 1977, 1979, 1981, 1984, 1987, 1988, 1990, 1994, 1998, } \\
2001,2005,2007,2011,2015\end{array}$ \\
\hline Spain & 1977, 1979, 1982, 1986, 1989, 1993, 1996, 2000, 2004, 2008, 2011, 2015, 2016 \\
\hline Finland & 1970, 1972, 1975, 1979, 1983, 1987, 1991, 1995, 1999, 2003, 2007, 2011, 2015 \\
\hline France & $1968,1973,1978,1981,1986,1988,1993,1997,2002,2007,2012$ \\
\hline United Kir & $1970,1974,1979,1983,1987,1992,1997,2001,2005,2010,2015$ \\
\hline Greece & $\begin{array}{l}1974,1977,1981,1985,1989,1990,1993,1996,2000,2004,2007,2009,2012, \\
2015\end{array}$ \\
\hline Ireland & 1969, 1973, 1977, 1981, 1982, 1987, 1989, 1992, 1997, 2002, 2007, 2011, 2016 \\
\hline Italy & $1968,1972,1976,1979,1983,1987,1992,1994,1996,2001,2006,2008,2013$ \\
\hline Japan & $\begin{array}{l}\text { 1969, 1972, 1976, 1979, 1980, 1983, 1986, 1990, 1993, 1996, 2000, 2003, 2005, } \\
2009,2012,2014\end{array}$ \\
\hline Netherlands & $\begin{array}{l}\text { 1967, 1971, 1972, 1977, 1981, 1982, 1986, 1989, 1994, 1998, 2002, 2003, 2006, } \\
2010,2012\end{array}$ \\
\hline Norway & $1969,1973,1977,1981,1985,1989,1993,1997,2001,2005,2009,2013$ \\
\hline Portugal & $\begin{array}{l}1975,1976,1979,1980,1983,1985,1987,1991,1995,1999,2002,2005,2009, \\
2011,2015\end{array}$ \\
\hline Sweden & $\begin{array}{l}1970,1973,1976,1979,1982,1985,1988,1991,1994,1998,2002,2006,2010, \\
2014\end{array}$ \\
\hline United States & $\begin{array}{l}1970,1972,1974,1976,1978,1980,1980,1982,1984,1986,1988,1990,1992, \\
1994,1996,1998,2000,2002,2004,2006,2008,2010,2012,2014,2016\end{array}$ \\
\hline
\end{tabular}

There are 291 parliamentary elections in total. The data was compiled by Döring and Manow (2015।. Dictatorial regimes, i.e. Spain 1970-1976, Greece 1970-1973 and Portugal 1970-1974, are not considered throughout this paper. 


\section{Far-left and far-right Parties from 1970 to 2016}

\begin{tabular}{|c|c|c|}
\hline \multirow[t]{2}{*}{ Australia } & Right & Australia First, Citizens Electoral Council, One Nation, Rise Up Australia \\
\hline & Left & $\begin{array}{l}\text { Communist Party of Australia, Democratic Socialist Electoral League, Democratic Socialist Perspective, Socialist } \\
\text { Alliance }\end{array}$ \\
\hline \multirow[t]{2}{*}{ Austria } & Right & Alliance for the Future of Austria, Freedom Party of Austria, Movement for Political Renewal \\
\hline & Left & Socialist Left Party, Communist Party of Austria \\
\hline \multirow[t]{2}{*}{ Belgium } & Right & Flemish Block, Flemish Interest, Libertarian-Direct-Democratic, National Front, People's Party, People's Union \\
\hline & Left & Communist Party of Belgium, Left Socialist Party, Worker's Party of Belgium \\
\hline \multirow[t]{2}{*}{ Canada } & Right & No parties identified \\
\hline & Left & Communist Party of Canada, Communist Party of Canada - Marxist-Leninst \\
\hline \multirow[t]{2}{*}{ Switzerland } & Right & Freedom Party of Switzerland, Geneva Citizens' Movement, Swiss Democrats, Swiss People's Party, Ticino League \\
\hline & Left & $\begin{array}{l}\text { Alternative Left, Autonomous Socialist Party, Progressive Organizations of Switzerland, Solidarity, Swiss Party of } \\
\text { Labour }\end{array}$ \\
\hline \multirow[t]{2}{*}{ Germany } & Right & $\begin{array}{l}\text { Alternative for Germany, Civil Rights Movement Solidarity, German Party, German People's Union, Law and Order } \\
\text { Offensive, National Democratic Party of Germany, Patriots for Germany, Popular Vote, Pro Germany, Pro German } \\
\text { Middle, Statt Party, The Offensive }\end{array}$ \\
\hline & Left & $\begin{array}{l}\text { Action Democratic Progress, Alliance of Germans, Collection to Actions, German Communist Party, German Union } \\
\text { for Peace, Marxist-Leninist Party of Germany, The Left }\end{array}$ \\
\hline \multirow[t]{2}{*}{ Denmark } & Right & Danish People's Party, Progress Party \\
\hline & Left & $\begin{array}{l}\text { Communist Party of Denmark, Common Course, Left Socialists, Socialist People's Party, Unity List-Red Green } \\
\text { Alliance }\end{array}$ \\
\hline \multirow[t]{2}{*}{ Spain } & Right & Basque Left, Basque Nationalist Party \\
\hline & Left & Communist Party of Spain, We Can, In Common We Can, Workers' Party of Marxist Unification, United Left \\
\hline \multirow{2}{*}{ Finland } & Right & Finns Party, Finnish Rural Party \\
\hline & Left & Communist Worker's Party, Communist Party of Finland, Finnish People's Democratic League, Left Alliance \\
\hline \multirow[t]{2}{*}{ France } & Right & Movement for France, National Front, National Republican Movement \\
\hline & Left & French Communist Party, Unified Socialist Party, Left Front, Revolutionary Communist League, Worker's Struggle \\
\hline \multirow[t]{2}{*}{ United Kingdom } & Right & $\begin{array}{l}\text { British National Party, Democratic Unionist Party, English Democrats, National Democratic Party, National Front, } \\
\text { United Kingdom Independence Party }\end{array}$ \\
\hline & Left & $\begin{array}{l}\text { Communist Party of Great Britain, Green Party of England and Wales, Plaid Cymru, Respect Party, Scottish Socialist } \\
\text { Party, Sinn Féin, Socialist Alternative, Socialist Labor Party }\end{array}$ \\
\hline \multirow[t]{2}{*}{ Greece } & Right & Golden Dawn, Independent Greeks, National Democratic Union, National Political Union, Popular Orthodox Rally \\
\hline & Left & $\begin{array}{l}\text { Coalition of the Radical Left, Communist Party of Greece, Communist Party of Greece (Interior), Democratic Left, } \\
\text { Synaspismos, United Democratic Left }\end{array}$ \\
\hline \multirow[t]{2}{*}{ Ireland } & Right & No parties identified \\
\hline & Left & $\begin{array}{l}\text { Communist Party of Ireland, Democratic Left, People Before Profit Alliance, Sinn Féin, Socialist Labour Party, } \\
\text { Socialist Party, Workers Party }\end{array}$ \\
\hline \multirow[t]{2}{*}{ Italy } & Right & $\begin{array}{l}\text { Brothers of Italy, Casa Pound, Italian Social Movement, National Alliance, New Force, No Euro, Northern League, } \\
\text { Social Alternative, The Freedomites, The Right, Tricolour Flame }\end{array}$ \\
\hline & Left & $\begin{array}{l}\text { Civil Revolution, Communist Refoundation Party, Communist Worker's Party, Critical Left, Democratic Party of the } \\
\text { Left, Five Star Movement, Italian Communist Party, Party of Italian Communists }\end{array}$ \\
\hline \multirow[t]{2}{*}{ Japan } & Right & Japan Restauration Party \\
\hline & Left & Japanese Communist Party \\
\hline \multirow[t]{2}{*}{ Netherlands } & Right & $\begin{array}{l}\text { Centre Democrats, Centre Party, Democratic Political Turning Point, Liveable Netherland, One NL, Party for Freedom, } \\
\text { Patriotic Democratic Appeal, Pim Fortuyn List, Proud of the Netherlands }\end{array}$ \\
\hline & Left & $\begin{array}{l}\text { Communist Party of the Netherlands, New Communist Party of the Netherlands, Pacifist Socialist Party, Socialist } \\
\text { Party }\end{array}$ \\
\hline \multirow[t]{2}{*}{ Norway } & Right & Democrats in Norway, Fatherland Party, Norwegian People's Party, Progress Party, The Democrats \\
\hline & Left & Communist Party of Norway, Socialist Left Party, The Red Party \\
\hline \multirow[t]{2}{*}{ Portugal } & Right & Democratic and Social Centre-People's Party, National Renovator Party \\
\hline & Left & $\begin{array}{l}\text { Unified Democratic Coalition, Bloc of the Left, Left Revolutionary Front, People's Democratic Union, People's Socialist } \\
\text { Front, Portuguese Communist Party, Portuguese Labour Party, Portuguese Workers' Communist Party, Revolutionary } \\
\text { Socialist Party, United People Alliance, Workers Party of Socialist Unity }\end{array}$ \\
\hline \multirow[t]{2}{*}{ Sweden } & Right & New Democracy, Sweden Democrats \\
\hline & Left & Communist Party of Sweden, The Left Party \\
\hline \multirow[t]{2}{*}{ United States } & Right & No parties identified \\
\hline & Left & No parties identified \\
\hline
\end{tabular}




\section{Recessions}

\begin{tabular}{l|l}
\hline \hline Country & Year \\
\hline Australia & $1971,1975,1977,1981,1990$ \\
\hline Austria & $1974,1980,1982,1983,1992,2000,2008,2012$ \\
\hline Belgium & $1974,1976,1980,1992,2000,2008,2012$ \\
\hline Canada & $1974,1980,1981,1990,2008,2014$ \\
\hline Switzerland & $1974,1977,1981,1990,1992,1994,1996,1998,2002,2008$ \\
\hline Germany & $1974,1980,1982,1992,1995,2001,2002,2008,2012$ \\
\hline Denmark & $1973,1977,1980,1987,1990,1992,1997,2001,2006,2007,2011,2015$ \\
\hline Spain & $1974,1978,1980,1992,2008,2010$ \\
\hline Finland & $1975,1976,1980,1990,2007,2012,2013$ \\
\hline France & $1974,1980,1992,2008$ \\
\hline UK & $1973,1974,1979,1990,2008$ \\
\hline Greece & $1973,1976,19980,1981,1985,1990,1992,1992,2007,2014$ \\
\hline Ireland & $1975,1982,1985,2007,2012,2016$ \\
\hline Italy & $1974,1977,1981,1992,1997,2001,2002,2008,2011,2013$ \\
\hline Japan & $1973,1993,1997,2001,2008,2010,2012,2014$ \\
\hline Netherlands & $1973,1074,1976,1979,1982,2003,2008,2011$ \\
\hline Norway & $1980,1981,1987,1992,2002,2007,2010,2015$ \\
\hline Portugal & $1974,1980,1983,1992,2002,2008,2010$ \\
\hline Sweden & $1970,1976,1980,1990,2007,2011$ \\
\hline USA & $1973,1980,1981,1990,2007$ \\
\hline \hline
\end{tabular}

We use quarterly real GDP per capita from the Federal Reserve Bank of St. Louis to identify the peaks and troughs of economic activity for each country by applying the algorithm of Bry and Boschan (1971). 


\section{Systemic Financial Crises}

\begin{tabular}{l|l}
\hline \hline Country & Year \\
\hline Australia & 1989 \\
\hline Austria & 2008 \\
\hline Belgium & 2008,2012 \\
\hline Switzerland & 1991,2008 \\
\hline Germany & 2008 \\
\hline Denmark & 1987,2008 \\
\hline Spain & 1977,2008 \\
\hline Finland & 1970 \\
\hline France & 2008 \\
\hline UK & $1974,1991,2007$ \\
\hline Greece & 2008 \\
\hline Ireland & 2008 \\
\hline Italy & $1990,2008,2011,2013$ \\
\hline Japan & 1997 \\
\hline Netherlands & 2008 \\
\hline Norway & 1988 \\
\hline Portugal & 2008 \\
\hline Sweden & 1991,2008 \\
\hline USA & 1984,2007 \\
\hline \hline
\end{tabular}

This refers to a systematic crisis characterized by government intervention. This dataset is drawn from Jordà et al. (2017). 
Summary Statistics

\begin{tabular}{lccccc}
\hline \hline \multicolumn{1}{c}{ Variable } & Mean & Std. Dev. & Min. & Max. & N \\
\hline Far-left vote share & 0.053 & 0.071 & 0 & 0.533 & 291 \\
Far-right vote share & 0.052 & 0.075 & 0 & 0.359 & 291 \\
Middle vote share & 0.896 & 0.11 & 0.373 & 1 & 291 \\
Government vote share & 0.541 & 0.122 & 0.123 & 0.865 & 291 \\
Opposition vote share & 0.459 & 0.122 & 0.135 & 0.877 & 291 \\
GiniMarket & 44.442 & 5.344 & 26.301 & 56.572 & 915 \\
GiniNet & 28.537 & 4.401 & 16.605 & 38.736 & 915 \\
Income share top 10\% & 24.73 & 2.23 & 20.1 & 30.8 & 241 \\
Income share top 20\% & 39.67 & 2.62 & 34.7 & 46.9 & 241 \\
Income share bottom 10\% & 3.02 & .591 & 1.7 & 4 & 241 \\
Income share bottom 20\% & 7.82 & 1.15 & 5 & 9.9 & 241 \\
Unemployment rate & 0.068 & 0.041 & 0.01 & 0.275 & 909 \\
RGDP growth & 0.018 & 0.023 & -0.09 & 0.096 & 904 \\
Inflation & 0.05 & 0.05 & -0.045 & 0.288 & 924 \\
Real house price growth & 0.02 & 0.069 & -0.177 & 0.388 & 838 \\
Gov. expenditure/GDP growth & 0.001 & 0.007 & -0.038 & 0.031 & 894 \\
Recession dummy & 0.319 & 0.466 & 0 & 1 & 924 \\
Financial crisis dummy & 0.03 & 0.172 & 0 & 1 & 924 \\
Globalization index & 75.487 & 12.425 & 36.661 & 92.848 & 924 \\
Economic glob. & 25.499 & 5.178 & 11.98 & 34.974 & 924 \\
Social glob. & 26.414 & 6.147 & 9.050 & 34.56 & 924 \\
Political glob. & 23.429 & 2.846 & 12.495 & 26.54 & 924 \\
Voter turnout & 0.764 & 0.127 & .422 & .9577 & 921 \\
\hline \hline
\end{tabular}




\section{Further Rolling Regressions Results}
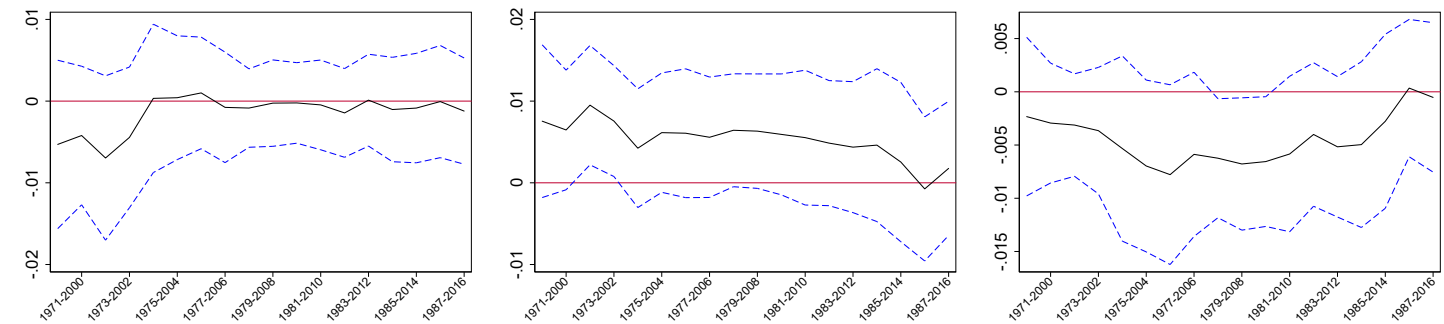

(a) EcoGlob. Coefficient on FLVS

(b) EcoGlob Coefficient on MVS

(c) EcoGlob. Coefficient on FRVS

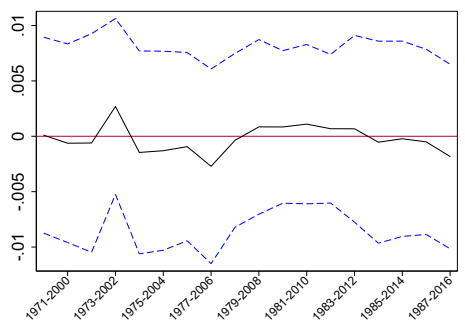

(d) PolGlob Coefficient on FLVS

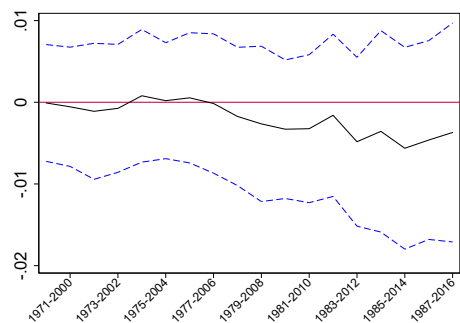

(e) PolGlob Coefficient on MVS

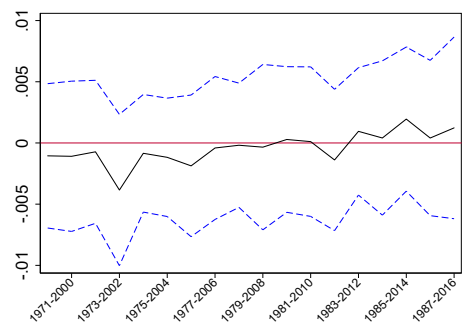

(f) PolGlob Coefficient on FRVS

Figure 2.4: Time-varying coefficients obtained from rolling panel regressions of FRVS and FLVS. 


\section{Further Tail Income Inequality Results}

Table 2.10: Panel OLS regressions with ratio 90_10 (all countries). Subsample: 2000-2016

\begin{tabular}{lcccccc}
\hline \hline & \multicolumn{2}{c}{ FLVS } & \multicolumn{2}{c}{ MVS } & \multicolumn{2}{c}{ FRVS } \\
& NTE & TE & NTE & TE & NTE & TE \\
\hline Ratio 90_10 $(t-1)$ & 0.182 & $0.230^{*}$ & $-0.246^{* *}$ & $-0.295^{*}$ & 0.175 & 0.199 \\
Unemployment rate $(t-1)$ & $0.690^{* *}$ & $0.841^{* * *}$ & $-0.544^{* * *}$ & $-0.675^{* * *}$ & 0.131 & 0.176 \\
RGDP growth $(t-1)$ & $-0.225^{* * *}$ & $-0.193^{* * *}$ & $0.238^{* * *}$ & $0.260^{* * *}$ & -0.125 & $-0.183^{*}$ \\
Inflation $(t-1)$ & 0.008 & 0.151 & -0.011 & -0.109 & 0.007 & 0.015 \\
Credit HH/GDP growth $(t-1)$ & 0.019 & -0.048 & 0.057 & 0.106 & -0.095 & -0.103 \\
Real house price growth $(t-1)$ & 0.115 & $0.177^{* * *}$ & -0.134 & $-0.245^{* *}$ & 0.082 & 0.177 \\
Gov. exp/GDP growth $(t-1)$ & $-0.189^{* *}$ & $-0.216^{* *}$ & 0.104 & 0.091 & 0.026 & 0.067 \\
Recession dummy $(t-1)$ & -0.013 & -0.009 & 0.079 & 0.106 & -0.096 & -0.136 \\
Financial crisis dummy $(t-1)$ & $0.086^{* *}$ & 0.021 & $-0.105^{* *}$ & 0.056 & 0.067 & -0.095 \\
Economic glob. $(t-1)$ & -0.172 & 0.032 & 0.120 & -0.165 & -0.012 & 0.197 \\
Social glob. $(t-1)$ & 0.088 & 0.112 & -0.022 & -0.049 & -0.048 & -0.032 \\
Political glob. $(t-1)$ & -0.073 & -0.082 & -0.017 & -0.042 & 0.087 & 0.130 \\
\hline Observations & 80 & 80 & 80 & 80 & 80 & 80 \\
\hline \hline
\end{tabular}

Standardized beta coefficients, ${ }^{*} p<0.10,{ }^{* *} p<0.05,{ }^{* * *} p<0.01$ significance levels 
Table 2.11: Panel OLS regressions with income share ratio 80_20 (all countries). Sample: 1970-2016

\begin{tabular}{lcccccc}
\hline \hline & \multicolumn{2}{c}{ FLVS } & \multicolumn{2}{c}{ MVS } & \multicolumn{2}{c}{ FRVS } \\
& NTE & TE & NTE & TE & NTE & TE \\
\hline Ratio 80_20 $(t-1)$ & $0.282^{*}$ & 0.253 & $-0.360^{* *}$ & $-0.306^{*}$ & $0.267^{* * *}$ & 0.216 \\
Unemployment rate $(t-1)$ & $0.432^{* *}$ & $0.809^{* * *}$ & $-0.291^{*}$ & $-0.576^{* * *}$ & 0.040 & 0.119 \\
RGDP growth $(t-1)$ & $-0.191^{* * *}$ & $-0.224^{* * *}$ & $0.214^{* * *}$ & $0.260^{* * *}$ & -0.138 & -0.175 \\
Inflation $(t-1)$ & -0.054 & $0.167^{*}$ & 0.051 & -0.095 & -0.026 & -0.010 \\
Credit HH/GDP growth $(t-1)$ & -0.084 & -0.057 & 0.049 & 0.082 & 0.003 & -0.067 \\
Real house price growth $(t-1)$ & 0.066 & $0.183^{* * *}$ & -0.068 & $-0.211^{*}$ & 0.039 & 0.142 \\
Gov. exp/GDP growth $(t-1)$ & -0.147 & $-0.227^{* *}$ & 0.084 & 0.110 & 0.007 & 0.041 \\
Recession dummy $(t-1)$ & 0.004 & -0.011 & 0.069 & 0.098 & -0.100 & -0.130 \\
Financial crisis dummy $(t-1)$ & 0.050 & 0.015 & -0.058 & 0.047 & 0.039 & -0.080 \\
Economic glob. $(t-1)$ & -0.264 & -0.041 & $0.283^{*}$ & 0.087 & -0.173 & -0.089 \\
Social glob. $(t-1)$ & -0.018 & 0.178 & -0.109 & -0.134 & $0.171^{*}$ & 0.037 \\
Political glob. $(t-1)$ & 0.029 & -0.257 & 0.006 & 0.091 & -0.034 & 0.093 \\
\hline Observations & 108 & 108 & 108 & 108 & 108 & 108 \\
\hline \hline
\end{tabular}

Standardized beta coefficients, ${ }^{*} p<0.10,{ }^{* *} p<0.05,{ }^{* * *} p<0.01$ significance levels

Table 2.12: Panel OLS regressions with ratio 80_20 (all countries). Sample: 2000-2016

\begin{tabular}{lcccccc}
\hline \hline & \multicolumn{2}{c}{ FLVS } & \multicolumn{2}{c}{ MVS } & \multicolumn{2}{c}{ FRVS } \\
& NTE & TE & NTE & TE & NTE & TE \\
\hline Ratio 80_20 $(t-1)$ & 0.209 & 0.218 & $-0.292^{* *}$ & $-0.328^{*}$ & 0.214 & 0.255 \\
Unemployment rate $(t-1)$ & $0.695^{* *}$ & $0.860^{* * *}$ & $-0.547^{* * *}$ & $-0.695^{* * *}$ & 0.131 & 0.188 \\
RGDP growth $(t-1)$ & $-0.228^{* * *}$ & $-0.197^{* * *}$ & $0.242^{* * *}$ & $0.263^{* * *}$ & -0.129 & $-0.185^{*}$ \\
Inflation $(t-1)$ & 0.016 & 0.163 & -0.019 & -0.133 & 0.012 & 0.037 \\
Credit HH/GDP growth $(t-1)$ & 0.020 & -0.049 & 0.053 & 0.096 & -0.090 & -0.088 \\
Real house price growth $(t-1)$ & 0.115 & $0.181^{* * *}$ & -0.133 & $-0.246^{* *}$ & 0.079 & 0.175 \\
Gov. exp/GDP growth $(t-1)$ & $-0.193^{* *}$ & $-0.207^{* *}$ & 0.110 & 0.090 & 0.020 & 0.061 \\
Recession dummy $(t-1)$ & -0.014 & -0.017 & 0.080 & 0.111 & -0.097 & -0.137 \\
Financial crisis dummy $(t-1)$ & $0.089^{* *}$ & 0.022 & $-0.109^{* *}$ & 0.049 & 0.071 & -0.086 \\
Economic glob. $(t-1)$ & -0.183 & 0.039 & 0.140 & -0.165 & -0.028 & 0.191 \\
Social glob. $(t-1)$ & 0.104 & 0.140 & -0.045 & -0.097 & -0.031 & 0.009 \\
Political glob. $(t-1)$ & -0.086 & -0.090 & 0.006 & -0.009 & 0.068 & 0.092 \\
\hline Observations & 80 & 80 & 80 & 80 & 80 & 80 \\
\hline \hline
\end{tabular}

Standardized beta coefficients, ${ }^{*} p<0.10,{ }^{* *} p<0.05, * * * p<0.01$ significance levels 
Table 2.13: Panel OLS regressions with bottom 20\% income share (all countries). Sample: 1970-2016

\begin{tabular}{lcccccc}
\hline \hline & \multicolumn{2}{c}{ FLVS } & \multicolumn{2}{c}{ MVS } & \multicolumn{2}{c}{ FRVS } \\
& NTE & TE & NTE & TE & NTE & TE \\
\hline Inc. share bottom $20(t-1)$ & -0.244 & -0.260 & $0.383^{* *}$ & $0.379^{* *}$ & $-0.333^{* * *}$ & $-0.314^{*}$ \\
Unemployment rate $(t-1)$ & $0.434^{* *}$ & $0.806^{* * *}$ & $-0.285^{*}$ & $-0.568^{* * *}$ & 0.029 & 0.110 \\
RGDP growth $(t-1)$ & $-0.186^{* * *}$ & $-0.217^{* * *}$ & $0.204^{* *}$ & $0.249^{* * *}$ & -0.129 & -0.166 \\
Inflation $(t-1)$ & -0.038 & $0.172^{*}$ & 0.039 & -0.111 & -0.023 & 0.009 \\
Credit HH/GDP growth $(t-1)$ & -0.125 & -0.098 & 0.090 & 0.113 & -0.020 & -0.075 \\
Real house price growth $(t-1)$ & 0.083 & $0.192^{* * *}$ & -0.086 & $-0.219^{* *}$ & 0.051 & 0.145 \\
Gov. exp/GDP growth $(t-1)$ & -0.122 & $-0.200^{*}$ & 0.055 & 0.093 & 0.027 & 0.041 \\
Recession dummy $(t-1)$ & 0.004 & -0.007 & 0.065 & 0.091 & -0.095 & -0.123 \\
Financial crisis dummy $(t-1)$ & 0.048 & 0.030 & -0.056 & 0.020 & 0.038 & -0.054 \\
Economic glob. $(t-1)$ & -0.237 & -0.020 & 0.259 & 0.063 & -0.163 & -0.071 \\
Social glob. $(t-1)$ & -0.015 & 0.142 & -0.117 & -0.107 & $0.180^{*}$ & 0.029 \\
Political glob. $(t-1)$ & -0.010 & -0.304 & 0.073 & 0.185 & -0.095 & -0.001 \\
\hline Observations & 108 & 108 & 108 & 108 & 108 & 108 \\
\hline \hline
\end{tabular}

Standardized beta coefficients, ${ }^{*} p<0.10,{ }^{* *} p<0.05,{ }^{* * *} p<0.01$ significance levels

Table 2.14: Panel OLS regressions with bottom 20\% income share (all countries). Subsample: 20002016

\begin{tabular}{lcccccc}
\hline \hline & \multicolumn{2}{c}{ FLVS } & \multicolumn{2}{c}{ MVS } & \multicolumn{2}{c}{ FRVS } \\
& NTE & TE & NTE & TE & NTE & TE \\
\hline Inc. share bottom $20(t-1)$ & -0.206 & -0.182 & $0.378^{* * *}$ & $0.396^{*}$ & $-0.334^{*}$ & -0.380 \\
Unemployment rate $(t-1)$ & $0.712^{* *}$ & $0.843^{* * *}$ & $-0.542^{* * *}$ & $-0.650^{* * *}$ & 0.110 & 0.141 \\
RGDP growth $(t-1)$ & $-0.218^{* * *}$ & $-0.195^{* * *}$ & $0.227^{* * *}$ & $0.253^{* * *}$ & -0.116 & $-0.173^{*}$ \\
Inflation $(t-1)$ & 0.015 & 0.131 & -0.001 & -0.091 & -0.011 & 0.007 \\
Credit HH/GDP growth $(t-1)$ & 0.019 & -0.069 & 0.025 & 0.102 & -0.051 & -0.078 \\
Real house price growth $(t-1)$ & 0.123 & $0.182^{* * *}$ & -0.128 & $-0.237^{* *}$ & 0.066 & 0.162 \\
Gov. exp/GDP growth $(t-1)$ & $-0.184^{* *}$ & $-0.192^{*}$ & 0.115 & 0.087 & 0.005 & 0.052 \\
Recession dummy $(t-1)$ & -0.013 & -0.019 & 0.072 & 0.104 & -0.088 & -0.125 \\
Financial crisis dummy $(t-1)$ & $0.086^{* *}$ & 0.014 & $-0.111^{* *}$ & 0.046 & 0.076 & -0.076 \\
Economic glob. $(t-1)$ & -0.138 & 0.057 & 0.094 & -0.178 & -0.006 & 0.193 \\
Social glob. $(t-1)$ & 0.104 & 0.111 & -0.051 & -0.072 & -0.022 & -0.000 \\
Political glob. $(t-1)$ & -0.059 & -0.050 & 0.004 & -0.026 & 0.047 & 0.080 \\
\hline Observations & 80 & 80 & 80 & 80 & 80 & 80 \\
\hline \hline
\end{tabular}

Standardized beta coefficients, ${ }^{*} p<0.10,{ }^{* *} p<0.05,{ }^{* * *} p<0.01$ significance levels 
Table 2.15: Panel OLS regressions with bottom 20\% income share (European countries). Sample: 1970-2016

\begin{tabular}{lcccccc}
\hline \hline & \multicolumn{2}{c}{ FLVS } & \multicolumn{2}{c}{ MVS } & \multicolumn{2}{c}{ FRVS } \\
& NTE & TE & NTE & TE & NTE & TE \\
\hline Inc. share bottom $20(t-1)$ & -0.121 & -0.120 & $0.359^{*}$ & 0.607 & $-0.363^{* *}$ & -0.689 \\
Unemployment rate $(t-1)$ & $0.676^{* *}$ & $0.951^{* * *}$ & $-0.479^{*}$ & $-0.708^{* *}$ & 0.037 & 0.095 \\
RGDP growth $(t-1)$ & $-0.196^{* * *}$ & $-0.187^{* * *}$ & $0.259^{* * *}$ & $0.262^{* *}$ & -0.167 & -0.178 \\
Inflation $(t-1)$ & 0.100 & 0.138 & -0.050 & -0.159 & -0.022 & 0.087 \\
Credit HH/GDP growth $(t-1)$ & -0.129 & -0.013 & 0.119 & 0.079 & -0.043 & -0.092 \\
Real house price growth $(t-1)$ & $0.156^{*}$ & $0.201^{* *}$ & $-0.175^{*}$ & $-0.250^{* *}$ & 0.092 & 0.152 \\
Gov. exp/GDP growth $(t-1)$ & -0.045 & $-0.289^{*}$ & -0.006 & 0.073 & 0.048 & 0.156 \\
Recession dummy $(t-1)$ & -0.042 & -0.074 & 0.104 & 0.105 & -0.099 & -0.073 \\
Financial crisis dummy $(t-1)$ & 0.055 & -0.012 & -0.084 & 0.041 & 0.062 & -0.044 \\
Economic glob. $(t-1)$ & $-0.538^{* *}$ & -0.086 & $0.439^{*}$ & -0.537 & -0.104 & 0.777 \\
Social glob. $(t-1)$ & 0.221 & 0.145 & $-0.297^{* *}$ & -0.185 & $0.195^{* *}$ & 0.115 \\
Political glob. $(t-1)$ & 0.062 & -0.197 & 0.069 & 0.192 & -0.144 & -0.079 \\
\hline Observations & 79 & 79 & 79 & 79 & 79 & 79 \\
\hline \hline
\end{tabular}

Standardized beta coefficients, ${ }^{*} p<0.10,{ }^{* *} p<0.05,{ }^{* * *} p<0.01$ significance levels

Table 2.16: Panel OLS regressions with bottom 20\% income share (European countries). Subsample: 2000-2016

\begin{tabular}{lcccccc}
\hline \hline & \multicolumn{2}{c}{ FLVS } & \multicolumn{2}{c}{ MVS } & \multicolumn{2}{c}{ FRVS } \\
& NTE & TE & NTE & TE & NTE & TE \\
\hline Inc. share bottom $20(t-1)$ & -0.152 & -0.105 & $0.407^{* *}$ & $0.568^{*}$ & $-0.382^{*}$ & $-0.630^{*}$ \\
Unemployment rate $(t-1)$ & $0.848^{* *}$ & $0.915^{* * *}$ & $-0.670^{* * *}$ & $-0.725^{* *}$ & 0.086 & 0.096 \\
RGDP growth $(t-1)$ & $-0.264^{* * *}$ & $-0.183^{* * *}$ & $0.289^{* * *}$ & $0.272^{* * *}$ & -0.129 & -0.181 \\
Inflation $(t-1)$ & 0.048 & 0.111 & -0.005 & -0.136 & -0.038 & 0.073 \\
Credit HH/GDP growth $(t-1)$ & 0.052 & -0.012 & 0.015 & 0.083 & -0.067 & -0.094 \\
Real house price growth $(t-1)$ & $0.192^{*}$ & $0.196^{* *}$ & $-0.198^{*}$ & $-0.259^{* *}$ & 0.078 & 0.154 \\
Gov. exp/GDP growth $(t-1)$ & $-0.187^{* *}$ & $-0.275^{*}$ & 0.124 & 0.075 & 0.011 & 0.155 \\
Recession dummy $(t-1)$ & -0.040 & -0.071 & 0.087 & 0.107 & -0.075 & -0.072 \\
Financial crisis dummy $(t-1)$ & $0.096^{* *}$ & -0.012 & $-0.136^{* *}$ & 0.045 & 0.086 & -0.047 \\
Economic glob. $(t-1)$ & -0.176 & -0.059 & 0.141 & -0.389 & -0.020 & 0.551 \\
Social glob. $(t-1)$ & 0.129 & 0.082 & -0.065 & -0.111 & -0.035 & 0.068 \\
Political glob. $(t-1)$ & -0.022 & -0.046 & -0.020 & 0.048 & 0.045 & -0.019 \\
\hline Observations & 67 & 67 & 67 & 67 & 67 & 67 \\
\hline \hline
\end{tabular}

Standardized beta coefficients, ${ }^{*} p<0.10,{ }^{* *} p<0.05,{ }^{* * *} p<0.01$ significance levels 
Table 2.17: Panel OLS regressions with top 10\% income share (all countries). Sample: 1970-2016

\begin{tabular}{lcccccc}
\hline \hline & \multicolumn{2}{c}{ FLVS } & \multicolumn{2}{c}{ MVS } & \multicolumn{2}{c}{ FRVS } \\
& NTE & TE & NTE & TE & NTE & TE \\
\hline Inc. share top $10(t-1)$ & 0.182 & 0.151 & $-0.218^{* *}$ & -0.157 & $0.152^{* *}$ & 0.092 \\
Unemployment rate $(t-1)$ & $0.475^{* *}$ & $0.860^{* * *}$ & $-0.345^{*}$ & $-0.631^{* * *}$ & 0.078 & 0.153 \\
RGDP growth $(t-1)$ & $-0.200^{* * *}$ & $-0.229^{* * *}$ & $0.224^{* *}$ & $0.265^{* * *}$ & -0.146 & -0.178 \\
Inflation $(t-1)$ & -0.025 & 0.149 & 0.013 & -0.071 & 0.003 & -0.029 \\
Credit HH/GDP growth $(t-1)$ & -0.113 & -0.110 & 0.090 & 0.158 & -0.030 & -0.130 \\
Real house price growth $(t-1)$ & 0.079 & $0.202^{* * *}$ & -0.085 & $-0.235^{* *}$ & 0.053 & 0.158 \\
Gov. exp/GDP growth $(t-1)$ & -0.133 & $-0.183^{* *}$ & 0.065 & 0.048 & 0.023 & 0.090 \\
Recession dummy $(t-1)$ & 0.004 & -0.002 & 0.070 & 0.090 & -0.102 & -0.125 \\
Financial crisis dummy $(t-1)$ & 0.039 & -0.017 & -0.044 & 0.084 & 0.029 & -0.105 \\
Economic glob. $(t-1)$ & -0.232 & -0.044 & 0.240 & 0.087 & -0.140 & -0.085 \\
Social glob. $(t-1)$ & -0.061 & 0.084 & -0.057 & -0.019 & 0.133 & -0.045 \\
Political glob. $(t-1)$ & 0.035 & -0.193 & -0.002 & 0.006 & -0.027 & 0.157 \\
\hline Observations & 108 & 108 & 108 & 108 & 108 & 108 \\
\hline \hline
\end{tabular}

Standardized beta coefficients, ${ }^{*} p<0.10,{ }^{* *} p<0.05,{ }^{* * *} p<0.01$ significance levels

Table 2.18: Panel OLS regressions with top 10\% income share (all countries). Subsample: 2000-2016

\begin{tabular}{lcccccc}
\hline \hline & \multicolumn{2}{c}{ FLVS } & \multicolumn{2}{c}{ MVS } & \multicolumn{2}{c}{ FRVS } \\
& NTE & TE & NTE & TE & NTE & TE \\
\hline Inc. share top $10(t-1)$ & 0.095 & 0.115 & -0.155 & -0.162 & 0.128 & 0.120 \\
Unemployment rate $(t-1)$ & $0.759^{* * *}$ & $0.911^{* * *}$ & $-0.632^{* * *}$ & $-0.769^{* * *}$ & 0.191 & 0.244 \\
RGDP growth $(t-1)$ & $-0.227^{* * *}$ & $-0.205^{* * *}$ & $0.242^{* * *}$ & $0.275^{* * *}$ & -0.130 & $-0.194^{*}$ \\
Inflation $(t-1)$ & 0.044 & 0.149 & -0.057 & -0.109 & 0.038 & 0.017 \\
Credit HH/GDP growth $(t-1)$ & -0.035 & -0.083 & 0.127 & 0.148 & -0.143 & -0.129 \\
Real house price growth $(t-1)$ & $0.146^{*}$ & $0.202^{* * *}$ & $-0.173^{*}$ & $-0.278^{* *}$ & 0.107 & 0.200 \\
Gov. exp/GDP growth $(t-1)$ & $-0.157^{* *}$ & $-0.176^{*}$ & 0.063 & 0.042 & 0.052 & 0.099 \\
Recession dummy $(t-1)$ & -0.013 & -0.017 & 0.076 & 0.113 & -0.092 & -0.139 \\
Financial crisis dummy $(t-1)$ & 0.073 & -0.016 & $-0.087^{*}$ & 0.105 & 0.054 & -0.129 \\
Economic glob. $(t-1)$ & -0.124 & 0.088 & 0.064 & -0.238 & 0.023 & 0.246 \\
Social glob. $(t-1)$ & 0.067 & 0.085 & 0.012 & -0.015 & -0.076 & -0.055 \\
Political glob. $(t-1)$ & -0.019 & -0.024 & -0.078 & -0.111 & 0.123 & 0.173 \\
\hline Observations & 80 & 80 & 80 & 80 & 80 & 80 \\
\hline \hline
\end{tabular}

Standardized beta coefficients, ${ }^{*} p<0.10,{ }^{* *} p<0.05, * * * p<0.01$ significance levels 
Table 2.19: Panel OLS regressions with top 20\% income share (all countries). Sample: 1970-2016

\begin{tabular}{lcccccc}
\hline \hline & \multicolumn{2}{c}{ FLVS } & \multicolumn{2}{c}{ MVS } & \multicolumn{2}{c}{ FRVS } \\
& NTE & TE & NTE & TE & NTE & TE \\
\hline Inc. share top $20(t-1)$ & 0.218 & 0.152 & $-0.276^{* *}$ & -0.200 & $0.203^{* *}$ & 0.152 \\
Unemployment rate $(t-1)$ & $0.471^{* *}$ & $0.845^{* * *}$ & $-0.340^{* *}$ & $-0.622^{* * *}$ & 0.076 & 0.153 \\
RGDP growth $(t-1)$ & $-0.198^{* * *}$ & $-0.229^{* * *}$ & $0.223^{* *}$ & $0.266^{* * *}$ & -0.145 & -0.180 \\
Inflation $(t-1)$ & -0.039 & 0.144 & 0.031 & -0.069 & -0.011 & -0.027 \\
Credit HH/GDP growth $(t-1)$ & -0.090 & -0.099 & 0.057 & 0.126 & -0.004 & -0.092 \\
Real house price growth $(t-1)$ & 0.066 & $0.194^{* * *}$ & -0.069 & $-0.224^{*}$ & 0.040 & 0.150 \\
Gov. exp/GDP growth $(t-1)$ & $-0.149^{*}$ & $-0.189^{* *}$ & 0.086 & 0.068 & 0.006 & 0.066 \\
Recession dummy $(t-1)$ & 0.002 & -0.010 & 0.071 & 0.097 & -0.102 & -0.128 \\
Financial crisis dummy $(t-1)$ & 0.042 & -0.015 & -0.047 & 0.084 & 0.031 & -0.107 \\
Economic glob. $(t-1)$ & -0.238 & -0.040 & 0.249 & 0.089 & -0.148 & -0.092 \\
Social glob. $(t-1)$ & -0.063 & 0.111 & -0.052 & -0.056 & 0.128 & -0.016 \\
Political glob. $(t-1)$ & 0.064 & -0.173 & -0.038 & -0.008 & -0.001 & 0.161 \\
\hline Observations & 108 & 108 & 108 & 108 & 108 & 108 \\
\hline \hline
\end{tabular}

Standardized beta coefficients, ${ }^{*} p<0.10,{ }^{* *} p<0.05,{ }^{* * *} p<0.01$ significance levels

Table 2.20: Panel OLS regressions with top 20\% income share (all countries). Subsample: 2000-2016

\begin{tabular}{lcccccc}
\hline \hline & \multicolumn{2}{c}{ FLVS } & \multicolumn{2}{c}{ MVS } & \multicolumn{2}{c}{ FRVS } \\
& NTE & TE & NTE & TE & NTE & TE \\
\hline Inc. share top 10 (t-1) & 0.116 & 0.119 & -0.198 & -0.213 & 0.167 & 0.185 \\
Unemployment rate (t-1) & $0.749^{* * *}$ & $0.903^{* * *}$ & $-0.614^{* * *}$ & $-0.770^{* * *}$ & 0.175 & 0.251 \\
RGDP growth (t-1) & $-0.228^{* * *}$ & $-0.205^{* * *}$ & $0.244^{* * *}$ & $0.275^{* * *}$ & -0.132 & $-0.194^{*}$ \\
Inflation (t-1) & 0.039 & 0.153 & -0.048 & -0.127 & 0.031 & 0.037 \\
Credit HH/GDP growth (t-1) & -0.024 & -0.073 & 0.107 & 0.124 & -0.125 & -0.105 \\
Real house price growth (t-1) & 0.139 & $0.198^{* * *}$ & $-0.160^{*}$ & $-0.271^{* *}$ & 0.095 & 0.195 \\
Gov. exp/GDP growth (t-1) & $-0.167^{* *}$ & $-0.183^{*}$ & 0.081 & 0.058 & 0.038 & 0.083 \\
Recession dummy (t-1) & -0.015 & -0.024 & 0.079 & 0.118 & -0.094 & -0.140 \\
Financial crisis dummy (t-1) & $0.076^{*}$ & -0.007 & $-0.092^{*}$ & 0.092 & 0.059 & -0.119 \\
Economic glob. (t-1) & -0.141 & 0.083 & 0.093 & -0.233 & -0.003 & 0.245 \\
Social glob. (t-1) & 0.072 & 0.101 & 0.005 & -0.044 & -0.070 & -0.030 \\
Political glob. (t-1) & -0.030 & -0.033 & -0.057 & -0.083 & 0.104 & 0.142 \\
\hline Observations & 80 & 80 & 80 & 80 & 80 & 80 \\
\hline \hline
\end{tabular}

Standardized beta coefficients, ${ }^{*} p<0.10,{ }^{* *} p<0.05,{ }^{* * *} p<0.01$ significance levels 
Table 2.21: Panel OLS regressions with top 10\% income share (European countries). Sample: 19702016

\begin{tabular}{lcccccc}
\hline \hline & \multicolumn{2}{c}{ FLVS } & \multicolumn{2}{c}{ MVS } & \multicolumn{2}{c}{ FRVS } \\
& NTE & TE & NTE & TE & NTE & TE \\
\hline Inc. share top $10(t-1)$ & 0.109 & 0.156 & $-0.195^{*}$ & -0.264 & $0.160^{* *}$ & 0.209 \\
Unemployment rate $(t-1)$ & $0.684^{* * *}$ & $0.995^{* * *}$ & $-0.525^{* *}$ & $-0.881^{* * *}$ & 0.089 & 0.284 \\
RGDP growth $(t-1)$ & $-0.206^{* * *}$ & $-0.195^{* * *}$ & $0.285^{* * *}$ & $0.298^{* * *}$ & -0.192 & $-0.220^{*}$ \\
Inflation $(t-1)$ & 0.102 & 0.168 & -0.076 & -0.173 & 0.010 & 0.079 \\
Credit HH/GDP growth $(t-1)$ & -0.127 & -0.050 & 0.139 & 0.126 & -0.070 & -0.122 \\
Real house price growth $(t-1)$ & 0.147 & $0.208^{* *}$ & -0.164 & $-0.289^{* *}$ & 0.086 & 0.196 \\
Gov. exp/GDP growth $(t-1)$ & -0.048 & -0.249 & -0.014 & 0.002 & 0.060 & 0.214 \\
Recession dummy $(t-1)$ & -0.046 & -0.073 & 0.129 & 0.137 & -0.128 & -0.115 \\
Financial crisis dummy $(t-1)$ & 0.046 & -0.053 & -0.060 & 0.131 & 0.039 & -0.125 \\
Economic glob. $(t-1)$ & $-0.516^{* *}$ & 0.155 & 0.393 & -0.669 & -0.063 & 0.738 \\
Social glob. $(t-1)$ & 0.191 & 0.121 & -0.258 & -0.049 & 0.170 & -0.041 \\
Political glob. $(t-1)$ & 0.076 & -0.234 & 0.013 & -0.034 & -0.082 & 0.248 \\
\hline Observations & 79 & 79 & 79 & 79 & 79 & 79 \\
\hline \hline
\end{tabular}

Standardized beta coefficients, ${ }^{*} p<0.10,{ }^{* *} p<0.05,{ }^{* * *} p<0.01$ significance levels

Table 2.22: Panel OLS regressions with top 10\% income share (European countries). Subsample: 2000-2016

\begin{tabular}{lcccccc}
\hline \hline & \multicolumn{2}{c}{ FLVS } & \multicolumn{2}{c}{ MVS } & \multicolumn{2}{c}{ FRVS } \\
& NTE & TE & NTE & TE & NTE & TE \\
\hline Inc. share top 10 (t-1) & 0.076 & 0.147 & -0.167 & -0.265 & 0.144 & 0.205 \\
Unemployment rate (t-1) & $0.882^{* * *}$ & $0.957^{* * *}$ & $-0.774^{* * *}$ & $-0.902^{* * *}$ & 0.189 & 0.285 \\
RGDP growth (t-1) & $-0.274^{* * *}$ & $-0.190^{* * *}$ & $0.313^{* * *}$ & $0.310^{* * *}$ & -0.151 & $-0.224^{*}$ \\
Inflation (t-1) & 0.070 & 0.136 & -0.069 & -0.148 & 0.024 & 0.066 \\
Credit HH/GDP growth (t-1) & 0.015 & -0.049 & 0.115 & 0.132 & -0.161 & -0.124 \\
Real house price growth (t-1) & $0.206^{* *}$ & $0.202^{* *}$ & $-0.242^{* *}$ & $-0.299^{* *}$ & 0.122 & 0.198 \\
Gov. exp/GDP growth (t-1) & $-0.167^{*}$ & $-0.237^{*}$ & 0.068 & 0.002 & 0.065 & 0.213 \\
Recession dummy (t-1) & -0.047 & -0.070 & 0.111 & 0.139 & -0.099 & -0.114 \\
Financial crisis dummy (t-1) & $0.086^{*}$ & -0.055 & $-0.108^{*}$ & 0.144 & 0.060 & -0.134 \\
Economic glob. (t-1) & -0.168 & 0.106 & 0.111 & -0.484 & 0.010 & 0.523 \\
Social glob. (t-1) & 0.106 & 0.069 & -0.012 & -0.030 & -0.081 & -0.024 \\
Political glob. (t-1) & 0.004 & -0.055 & -0.105 & -0.008 & 0.131 & 0.061 \\
\hline Observations & 67 & 67 & 67 & 67 & 67 & 67 \\
\hline \hline
\end{tabular}

Standardized beta coefficients, ${ }^{*} p<0.10,{ }^{* *} p<0.05,{ }^{* * *} p<0.01$ significance levels 
Table 2.23: Panel OLS regressions with top 20\% income share (European countries). Sample: 19702016

\begin{tabular}{lcccccc}
\hline \hline & \multicolumn{2}{c}{ FLVS } & \multicolumn{2}{c}{ MVS } & \multicolumn{2}{c}{ FRVS } \\
& NTE & TE & NTE & TE & NTE & TE \\
\hline Inc. share top $20(t-1)$ & 0.136 & 0.128 & $-0.252^{*}$ & -0.304 & $0.211^{* *}$ & 0.285 \\
Unemployment rate $(t-1)$ & $0.680^{* * *}$ & $0.991^{* * *}$ & $-0.516^{*}$ & $-0.883^{* * *}$ & 0.081 & 0.289 \\
RGDP growth $(t-1)$ & $-0.206^{* * *}$ & $-0.194^{* * *}$ & $0.285^{* * *}$ & $0.297^{* * *}$ & -0.193 & $-0.218^{*}$ \\
Inflation $(t-1)$ & 0.096 & 0.173 & -0.063 & -0.210 & -0.001 & 0.124 \\
Credit HH/GDP growth $(t-1)$ & -0.105 & -0.026 & 0.097 & 0.097 & -0.035 & -0.105 \\
Real house price growth $(t-1)$ & 0.137 & $0.207^{* *}$ & -0.144 & $-0.286^{* *}$ & 0.069 & 0.193 \\
Gov. exp/GDP growth $(t-1)$ & -0.065 & $-0.273^{*}$ & 0.018 & 0.032 & 0.033 & 0.195 \\
Recession dummy $(t-1)$ & -0.048 & -0.081 & 0.132 & 0.149 & -0.131 & -0.123 \\
Financial crisis dummy $(t-1)$ & 0.048 & -0.034 & -0.064 & 0.108 & 0.042 & -0.112 \\
Economic glob. $(t-1)$ & $-0.520^{* *}$ & 0.046 & 0.399 & -0.629 & -0.068 & 0.782 \\
Social glob. $(t-1)$ & 0.186 & 0.155 & $-0.245^{*}$ & -0.133 & 0.158 & 0.038 \\
Political glob. $(t-1)$ & 0.096 & -0.232 & -0.024 & 0.045 & -0.052 & 0.144 \\
\hline Observations & 79 & 79 & 79 & 79 & 79 & 79 \\
\hline \hline
\end{tabular}

Standardized beta coefficients, ${ }^{*} p<0.10,{ }^{* *} p<0.05,{ }^{* * *} p<0.01$ significance levels

Table 2.24: Panel OLS regressions with top 20\% income share (European countries). Subsample: 2000-2016

\begin{tabular}{lcccccc}
\hline \hline & \multicolumn{2}{c}{ FLVS } & \multicolumn{2}{c}{ MVS } & \multicolumn{2}{c}{ FRVS } \\
& NTE & TE & NTE & TE & NTE & TE \\
\hline Inc. share top $20(t-1)$ & 0.097 & 0.124 & -0.210 & -0.313 & 0.179 & 0.288 \\
Unemployment rate $(t-1)$ & $0.875^{* * *}$ & $0.954^{* * *}$ & $-0.761^{* * *}$ & $-0.904^{* * *}$ & 0.178 & 0.289 \\
RGDP growth $(t-1)$ & $-0.276^{* * *}$ & $-0.189^{* * *}$ & $0.317^{* * *}$ & $0.308^{* * *}$ & -0.154 & $-0.222^{*}$ \\
Inflation $(t-1)$ & 0.067 & 0.139 & -0.062 & -0.180 & 0.019 & 0.104 \\
Credit HH/GDP growth $(t-1)$ & 0.027 & -0.025 & 0.089 & 0.101 & -0.138 & -0.107 \\
Real house price growth $(t-1)$ & $0.200^{* *}$ & $0.202^{* *}$ & $-0.230^{* *}$ & $-0.296^{* *}$ & 0.113 & 0.196 \\
Gov. exp/GDP growth $(t-1)$ & $-0.177^{*}$ & $-0.260^{*}$ & 0.089 & 0.033 & 0.047 & 0.194 \\
Recession dummy $(t-1)$ & -0.048 & -0.078 & 0.113 & 0.151 & -0.101 & -0.123 \\
Financial crisis dummy $(t-1)$ & $0.089^{*}$ & -0.035 & $-0.115^{*}$ & 0.119 & 0.066 & -0.121 \\
Economic glob. $(t-1)$ & -0.183 & 0.031 & 0.144 & -0.456 & -0.017 & 0.554 \\
Social glob. $(t-1)$ & 0.110 & 0.088 & -0.022 & -0.080 & -0.072 & 0.022 \\
Political glob. $(t-1)$ & -0.007 & -0.054 & -0.083 & 0.011 & 0.113 & 0.035 \\
\hline Observations & 67 & 67 & 67 & 67 & 67 & 67 \\
\hline \hline
\end{tabular}

Standardized beta coefficients, ${ }^{*} p<0.10,{ }^{* *} p<0.05,{ }^{* * *} p<0.01$ significance levels 


\title{
Chapter 3
}

\author{
Are Some People More Equal than Others? \\ Experimental Evidence on Group Identity and \\ Income Inequality ${ }^{1}$
}

\footnotetext{
${ }^{1}$ This Chapter is based on joint work with Prof. Dr. Christian R. Proaño, Jun.-Prof. Dr. Joep Lustenhouwer and Jun.-Prof. Dr. Tomasz Makarewicz. This paper can be found in BERG Working Paper No. 168.
} 


\subsection{Introduction}

The dramatic increase in economic inequality around the world over the last decades has become a central issue in the current political debate. At the same time, structural geopolitical and economic shifts have led to even more pronounced migratory movements not only to the European Union and the United States, but also in other parts of the world. It has been argued that both developments have contributed to the increasing polarization of politics and rise of extreme parties (see e.g. Inglehart and Norris, 2016 and Alesina et al., 2019), but it is difficult to disentangle their individual effects. This is because both phenomena have often influenced each other and have appeared simultaneously. On the one hand, the legal and illegal movement of people across borders has increased over the last decades not only due to events such as the creation of the NAFTA and the expansion of the common labor market in the EU, but also due to the Syrian War, the Venezuelan economic collapse, and others. On the other hand, the 2008 Great Recession exacerbated the already increasing income and wealth inequality around the world (Atkinson et al., 2011, Stiglitz, 2013 Piketty 2014b).

An important example of the influence of both developments is the Brexit. After the 2004 expansion of the EU, there was a significant increase of immigration of citizens of the new member countries that settled in the UK in search of jobs (Sumption and Vargas-Silva, 2020). This caused a political backlash in the British politics, which, after the 2008 economic crisis and the following recession, culminated with the Brexit referendum that was ultimately won by the "Leave" camp with a narrow margin. A further consequence of this political melee and the narrow victory margin in the referendum was an increased polarization, in terms of political distance, between pro- and anti-EU voters, as well as between Scotland and other British constituent countries. The polls showed that the immigration was the most important argument for Leavers (Fisher and Renwick, 2018), but their specific motivation remains unclear: was it a straightforward dislike of the "foreigners", or rather an economic anxiety that these "aliens" will take over jobs from the native population, and put pressure on the already strained social safety programs, as discussed by Fetzer (2019). A similar question can be raised regarding Trump voters and their attitudes with respect to Latin-American migrants in the 2016 US presidential election or regarding some EU citizens who vocally opposed the influx of refugees primarily from the Syrian War in 2015.

In the current debate about the perception and acceptance of immigrants, not only the changes in society and social identity play an important role, but also the shifts in the distribution of income that arise because of the arrival of immigrants. In this paper, we design a laboratory experiment to separate the effects on the participants' social preferences of group identity from those of relative economic status and thus from economic inequality reasons. Moreover, we try to find out which of the two plays a larger role in the minds of the laboratory subjects. Further, we are interested in how attitudes may become more polarized due to these factors. To the best of our knowledge, no 
experimental study has been conducted that addresses the question whether political polarization and an unwillingness for redistribution are mainly driven by factors related to relative economic status, or rather by social identity and group formation.

In our laboratory experiment we elicit opinions and preferences for different political schemes in a controlled environment. We focus on a setting of labor market and unemployment insurance, which is both politically relevant and familiar for our subjects. In each treatment we study a pool of 28 subjects who are assigned to two different types which we interpret as "natives" (20 subjects) and "migrants" ( 8 subjects), respectively. However, we do not use these politically charged terms towards the subjects in the experiment and instead rely on the more neutral Green and Purple color framing. The most important difference between these two groups is that the natives (besides their obvious majority) enjoy political privilege by having exclusive voting rights on the type of social safety net that is implemented.

In particular, each treatment runs for 20 or 25 periods. At the beginning of each period, subjects are randomly split into four groups, each consisting of 5 natives and 2 migrants. Every subject then randomly draws a chance of being employed (which we treat as a representation of the relative economic position in the society), and the native subjects vote whether they want to impose in their group: a) no unemployment insurance, b) unemployment insurance for the whole group, or c) a "partial insurance" (only for some group members, which depends on the treatment).

We use a two-by-two experimental design involving four treatments that allows us to disentangle the two dimensions of our research problem: income inequality between native and immigrant populations on the one hand, and group identity on the other. In the first dimension, native subjects sample employment chances from the same distribution as the migrants (Equality treatments) or are guaranteed to sample better chances than the migrants (Inequality treatments). In the second dimension, native subjects are unaware of the division of the group into two types with different political representation (Grey treatments) - or to the contrary, are explicitly told about the existence of the "migrants" subjects (who were framed as Purple) and group identity is induced by a simple group exercise for the Green subjects (the "natives") before the start of the experiment (Colorful treatments). Furthermore, in the two Colorful treatments "partial insurance" is framed as covering only the Green subjects, while in the two Gray treatments it is framed as covering only those with an high enough employment chance. This gives us the unique opportunity to isolate the effects of group identity from that of income inequality on political polarization and willingness for social sharing.

We are interested in several research questions. First, do people care about income inequality or about their relative economic status when they decide upon an insurance system that also affects their peers? Second, are people motivated by the pure like or dislike of the "other" even if it has no discernible impact on their income? Third, is there a potential feedback between these two dimensions?

Our experimental results can be summarized by the following four findings. First, subjects in 
all treatments were at least to some extent motivated by the prospect of a higher expected personal net income: the higher the employment chance, the less likely they were to vote for a full or partial insurance scheme. At the same time, subjects with low employment chances were more likely to choose partial insurance (rather than full) if this insurance scheme would imply being covered with higher benefits at a lower taxation level. Secondly, subjects from the Inequality treatments (with a more beneficial ex ante distribution of employment chances) tended to behave, on average, in a less selfish fashion. Combining the first and the second finding, the most selfish behavior was exhibited by subjects who faced ex ante worse income distribution, but were lucky to sample the best employment chance. Thirdly - and contrary to popular political discourse - color revelation resulted in the native subjects reacting with compassion towards the migrants, which can be seen in higher voting rates for the full insurance scheme. Fourthly, the voting heterogeneity (i.e. voting polarization) was higher both when group identities were revealed (in a way that benefited the migrants) and when the native income distribution was equalized with that of the migrants (in a way that was to the detriment of the latter population).

To sum up, our experiment suggests a complex relationship between voting, income distribution and group identity, and by extension, migration. It seems that relative economic status has contradictory "average" and "marginal" effects: subjects tend to be more inclusive when they enjoy an ex ante more privileged relative income position (in terms of the range of employment chances that they sample from), but once they achieve an ex post position which is better given their ex ante distribution, they become more selfish. Group identity, on the other hand, tends to lead to more polarization, but in a way that can make the migrants better off.

The remainder of the paper proceeds as follows. We review the existing related literature in Section 3.2. Section 3.3 describes the design of the experiment. In Section 3.4 we discuss our testable hypotheses and experimental procedures, while the results are discussed in Section 3.5 Finally, Section 3.6 summarizes our findings.

\subsection{Literature Overview}

This paper is based on two strands of literature. The first strand is focused on a large body of research that investigates how monetary incentives influence people's decisions. Many of these studies have focused on how monetary incentives can be used as an instrument to increase individual's performance. One the one hand, a number of authors have reported the positive effects of monetary incentives in individual actions. ${ }^{2}$ For example, Schröder et al. (2013), Börsch-Supan et al. (2013) and Singer et al. (1999) demonstrate the positive impact of monetary incentives on response rates in surveys. Similarly,

\footnotetext{
${ }^{2}$ Since a full discussion of the effect of monetary incentives on individual's performance lies beyond the scope of this study, we limit this part providing some empirical evidence in selected fields. See for instance Bonner and Sprinkle (2002) for a thorough literature review.
} 
Caselli and Morelli (2004) and Ferraz and Finan (2009) show how monetary incentives improve the quality of the government. One the other hand, Bonner et al. (2000) and Camerer and Hogarth (1999) find no evidence that monetary incentives increase performance in management accounting and in an experiment with mixtures of incentives schemes, respectively. Taken together, empirical evidence suggests that the effect of monetary incentives on decision making at the individual level cannot be easily generalized across different settings.

The second strand of research is related to the link between immigration/ethnic diversity and preferences for redistribution. A large and growing body of literature has suggested a negative relationship between immigration or ethnic diversity and the preferences of individuals for redistribution. ${ }^{3}$ Much of the previous research has been conducted using observational data from surveys, see for instance Alesina and Ferrara (2005) and Stichnoth and Van der Straeten (2013) for an overview. The first contributions related to these questions are provided by Alesina et al. (2001), Luttmer (2001) and Lind (2007) for the United States using the General Social Survey. They find that the support for redistribution differs by racial preferences as African Americans tend to be much more supportive of welfare spending compared to White Americans. Furthermore, Alesina and Glaeser (2004) argue that ethnic heterogeneity is one of the explaining factors why United Sates and European countries share different welfare systems. They state that higher social heterogeneity, that results from relatively higher immigration rate, tends to hinder the scope of redistribution policies.

More recently, Alesina et al. (2019) assess native's perceptions and attitudes towards immigrants and how these characteristics influence their preferences over redistribution. They conduct a largescale survey as well as an online experiment in six developed countries (France, Germany, Italy, Sweden, the United Kingdom, and the United States). From the survey results, they find that natives, on average, overestimated the factual share of immigrants in all countries and furthermore believed that immigrants had lower levels of education and also lower income levels. Most natives also wrongly estimated the composition of immigrants, thinking that most of them came from the Middle East and North Africa and Muslim regions. Stronger negative misperceptions towards immigrants were found among those individuals, who possessed low levels of education or who worked together with immigrants. The experiment was based on three different treatments. The first treatment (priming treatment) provided no information on immigration. The second treatment (informational treatment) provided factual information on the size and origin of immigrants. Finally, the third treatment (anecdotal treatment) provided a (non-factual) narrative story about a hard-working immigrant. The authors find no impact of these treatments on the connection between immigration perceptions and support for redistributive policies. In an earlier paper, Senik et al. (2009) draw similar conclusions. They use data from the European Social Survey from 2002/2003 to explore the link between immigration and support for a welfare state and find a strong association between negative attitudes towards

\footnotetext{
${ }^{3}$ Redistribution policy in the literature, as well as in this paper is meant to describe the relationship between taxation payments and expenditures related to social benefits and public services.
} 
immigrants and less support for welfare programs.

Natural experiments have also been used to study this phenomenon. For instance, Dahlberg et al. (2012) investigate the impact of the refugee placement program in Swedish municipalities from 1985 to 1994 and demonstrate that a larger share of immigrants coming from non-OECD countries reduced support for redistribution (social benefits) policies. Moreover, the support for welfare programs depends on the degree to which immigrants are allowed to participate in democratic processes? While natives have the right to vote and take decisions based on their own preferences, immigrants, in general, do not share the same rights and cannot express themselves in elections. This approach is explored by Chevalier et al. (2018) who found a positive link between the presence of immigrants with voting rights and redistribution. They investigate the arrival of around eight million forced migrants in West Germany after World War II who in fact were Germans themselves - they were refugees from the territories that Germany lost to USSR, Poland and Czechoslovakia after 1945, and they had full voting rights. The authors show how this inflow of immigrants had a positive effects on welfare state and preferences for more redistribution.

The literature has also examined how high levels of inequality may affect individual preferences for redistribution via other-regarding preferences. ?, p. 94 state that an altruistic behavior, i.e. a "situation in which one agent cares also about the utility of somebody else", may emerge among some individuals when the level of income inequality is high. Using the General Social Survey for United States and the World Value Survey for international cross-country evidence, the authors show that individual socioeconomic status, perception of fairness are among the important determinants of preferences for redistribution. Moreover, Giuliano and Spilimbergo (2009) show that macroeconomic historical experiences affect preferences for redistribution. Macroeconomic shocks may render some individuals more risk-averse, support more redistribution policies, and adopt a left-wing political orientation. $^{4}$

Another important issue is how immigration may affect political preferences. Dustmann et al. (2019) highlight the effect of refugee migration on voting outcomes in municipal elections in Denmark from 1986 to 1998. They find that larger shares of refugees in the overall population? increased electoral support for right-center and anti-immigration parties at the expense of left-leaning parties. Tabellini (2020) analyzes the effects of European immigration to the United States between 1910 and 1930. The author demonstrates that the higher cultural distance between the established population and new European immigrants, the more likely a backlash against immigrants. As a consequence, the political discontent of the already established population was expressed by the choice of lower redistribution policies through voting in favor of more conservative legislators.

More recent attention has focused on the social identity theory as a key determinant of people's preferences over distribution. This theory was developed by Tafjel and Turner (1979) to explore the

\footnotetext{
${ }^{4}$ For a general review of the literature, see Dimick et al. (2018).
} 
psychological basis for intergroup behavior and intergroup conflict. According to the social identity theory, the nature of the intergroup relations is based on social categorizations with which people identify themselves and others. Thoughts, values, beliefs or whatever that may play an important role for categorization will develop a feeling of membership in a group. As a result, this categorization draws the borderline between in-groups and out-groups where "intergroup categorization leads to in-group favoritism and discrimination against the out-group" (Tafjel and Turner 1986, p. 282). Given the relevance of this psychological factor, a considerable amount of recent theoretical and experimental studies has incorporated the social identity theory into economic analysis as a key determinant of redistributional preferences. ${ }^{5}$

Several lines of research confirm the in-group favoritism and out-group discrimination. Among the experimental evidence, Hargreaves Heap and Zizzo (2009) examine how group membership affects individuals' behavior in an experimental trust game and find that insiders show not only a positive discrimination (more charity) towards themselves, but also a negative discrimination (less charity) towards outsiders. Similarly, Chen and Li (2009) find a positive effect of group identity on social preferences. They point out that participants show more charity and less envy when they are matched with in-group members compared to the out-group. Eckel and Grossman (2005) designed a repeatedplay public good game based on a team production problem to examine the effect of identity on team production. They find that "just being identified with a team is, alone, insufficient to overcome self-interest". Nonetheless, activities created to improve team identity contribute to higher levels of team cooperation. Haile et al. (2008) conduct a series of trust game experiments in South Africa to explore the effects of racial and income inequality on cooperativeness in society. Although they do no find evidence that either race nor income by itself can lead to discrimination, they observe that "the low income individuals of both races invest little in partnerships with a high income partner from the other race" (Haile et al., 2008, p. 3). They define this behavior as cross-racial envy.

Finally, competition for limited resources is another important characteristic that may heighten inter-group relations. Campbell (1965) proposed the "realistic group conflict theory" which states that intergroup conflict is based on conflicts of interests between groups, and it thus intensifies due to rivalry over scarce resources as jobs, power, wealth or status differences (changes in the perceived prestige). Some studies have investigated discrimination towards immigrants by analyzing labor market competition as a main determinant, in particular how economic shocks have affected only individual's political opinions, as well as how societies have been becoming more polarized. Majlesi et al. (2020) illustrate that U.S. counties with greater trade exposure have became more polarized by the election of

\footnotetext{
${ }^{5}$ Akerlof and Kranton (2000) was the seminal study that introduced the social identity theory into economic analysis using a model of behavior based on a set of social categories. The authors propose the inclusion of social identity or selfperception in a utility function as the main motivation for individual behavior. This framework is used to understand gender discrimination, the economics of poverty and social exclusion. See also Costa-Font and Cowell (2015) for an overview on how social identity has been measured in the literature and its effect on redistribution attitudes.
} 
more conservative legislators in the United States. Similar conclusions are obtained by Colantone and Stanig (2018) and Anelli et al. (2019) for Western European countries. Colantone and Stanig (2018) highlight how political beliefs of individuals who live in regions more exposed to trade competition shifted towards more right-leaning political parties. Anelli et al. (2019) find that areas with more exposure to automation support more nationalist parties. Similarly, Gennaioli and Tabellini (2019) provide a theoretical model where group identity is introduced in a political economy that is described by the nature of in-group vs out-group. They investigate how group formation is based on voter's belief and how this distances leads to more polarization. Similarly, we are interested in which voting behavior and political polarization may be affected by increasing competition in the domestic labor market.

\subsection{Experimental Design}

\subsubsection{Setup}

We consider a simple $2 \times 2$ design involving 4 treatments, as illustrated in Table 3.1 For each treatment, we ran one session with 28 subjects (optimized to the size of our laboratory). In all treatments, participants are permanently assigned one of two different types: Green and Purple. The 20 Green participants are meant to represent the native part of the population, while the 8 Purple participants represent the non-native (or migrant) part. In order not to bias the experimental results, we do not frame to the subjects the two groups as native versus immigrant populations, but use a more politically neutral color framing.

The experiment was run for 20 periods for the one (GI, see below Table 3.1) treatment and for 25 periods for the three other treatments. In each period $t$, the 28 subjects were randomly split into groups of 7 , which are always composed of 5 Green participants and 2 Purple participants. There were thus always 4 groups of seven participants, however, their composition changed in every period $t$.

The subjects were informed that the group size was fixed at 7, and additionally, in the two Colorful treatments the subjects knew that their group always consisted of 5 Green and 2 Purple participants (including themselves). Subjects knew about the group randomization, but were not informed which other participants are members of their group at any time. This allows our design to exclude groupspecific lock-in dynamics, an issue that could be studied in future experiments.

In the two treatments denoted as Gray treatments $(\mathrm{G})$, the existence of the two color types is not revealed to the subjects. In the two other treatments, denoted as Colorful treatments (C), the subjects are informed about the division into two color types are further primed to be consciously aware of this division during the experiment. Firstly, at the beginning of the session, Green subjects 
participated in a quick group formation game, which will be described later. Secondly, the presence of the small Purple minority of the subjects was highlighted by purple pieces of paper on the wall over the desks of these subjects, which were visible for all participants throughout the game.

Table 3.1: Treatments

\begin{tabular}{|c|c|c|}
\hline & Gray (G) & Colorful (C) \\
\hline Equality (E) & GE & CE \\
\hline Inequality (I) & GI & CI \\
\hline
\end{tabular}

In each period $t$, all participants had a random chance of being employed and thus receiving a high income. The second treatment dimension relates these random employment chances to the two color types, as illustrated in Figure 3.1. In the Inequality (I) treatments the two Purple participants (the "migrants") always draw their employment chances randomly and independently ${ }^{6}$ from the $\{30 \%, 40 \%\}$ set, while the five Green participants (the "natives") always draw their chances randomly and independently from the $\{50 \%, 60 \%, 70 \%, 80 \%, 90 \%\}$ set. This implies that in the I treatments the Green subjects can expect ex ante a visibly higher income than the Purple ones. In the Equality treatments (E), it is also the case that in each period two participants draw from $\{30 \%, 40 \%\}$ and five participants draw from $\{50 \%, 60 \%, 70 \%, 80 \%, 90 \%\}$. However the two 'unlucky' participants are now randomly chosen from the whole group of seven subjects and could be Purple as well as Green participants. Hence, the ex-ante expected employment chance of Purple participants is the same as that of Green participants in the E treatments and there is no inherent income inequality between the two types. ${ }^{7}$

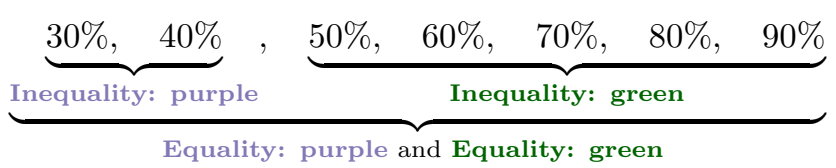

Figure 3.1: Employment probabilities

The task of all subjects in every period $t$ is to vote on an unemployment insurance scheme that can introduce a social security program in their group in the current period (the groups vote independently from each other). Subjects observe their accumulated payoff, the results from the last

\footnotetext{
${ }^{6}$ The independence means both across the subjects and the periods. All draws are taken from a uniform multinomial distribution.

${ }^{7}$ Notice that the in the two Inequality treatments, the Green subjects are in fact more equal between themselves, since they sample employment chances from a "better" and narrower distribution. The distinction between equality and inequality in this paper always refers to the between-group income differences.
} 
voting round that they had participated in, and their last payoff, as well as the employment chances of their current group members. The game has the following timing in each period $t$ :

1. if the period is $t \geqslant 2$, subjects are informed about the results of the voting from their former group, their payoff from period $t-1$ and their accumulated payoff;

2. subjects are notified about their own employment chances for the current period $t$, as well as employment chances of other members of their current group; 8

3. subjects vote individually on the insurance scheme, which is then selected based on the votes of the Green participants only (see Section 3.3.3);

4. the employment status of the subjects is realized based on their individual employment chances;

5. subject payoffs are realized based on their employment status and unemployment insurance.

We discuss the possible unemployment insurance schemes in Section 3.3.2 and then the voting mechanism in Section 3.3.3. In Section 3.3.4 we summarize the four treatments and highlight how these can be used to disentangle the effects of group identity from those of income equality. Payoffs are discussed in Sections 3.3.5. Finally, in Section 3.3.6 we discuss the short group formation game that we let Green subjects play to prime group identity in the Colorful treatments.

\subsubsection{Unemployment Insurance Scheme}

As previously mentioned, all the subjects observe their employment chance, as well as the employment chances of the other members of their current group at the beginning of each period $t$. Participants are then asked to vote for their first and second most preferred unemployment insurance scheme. The three possible choices are:

- Full insurance where the government provides a universal safety net for all 7 group members.

- Partial insurance where the government provides a safety net, but only for a specific part of the group (to be specified in detail below).

- No insurance where the government does nothing and no group member is insured.

Notice that with three options, eliciting the first and second choices yields the full ranking of preferences.

\footnotetext{
${ }^{8}$ Recall that groups are reshuffled in every period and that subjects are not informed about the identity of the members of their group. This also implies that they don't know the history of employment chances or of voting patterns of their current group peers.
} 
In the case that the Full insurance scheme is implemented in a given group, there is an active intervention of the government: all employed group participant (Green and Purple) pay a tax, while all unemployed group participants (Green and Purple) receive unemployment benefits. This happens irrespective of the treatment.

In the case that the No insurance scheme is implemented, there is no intervention of the government, irrespective of the treatment. As a result, there are neither tax payments nor unemployment benefits in this group.

Unlike for the other two options, the implementation of the Partial insurance scheme depends on the treatment. Under the Colorful (C) treatments, only Green subjects take part in the Partial insurance scheme, meaning that only the employed Green subjects pay the tax and only the unemployed Green participants receive the benefit. On the other hand, the Purple subjects are excluded. Note that under the Colorful inequality (CI) treatment, the Green participants have a monetary incentive to exclude the Purple ones, since these always have substantially lower employment chances. On the other hand, under the Colorful equality $(\mathrm{CE})$ treatment there is no such incentive ex ante, and there is only $1 / 42 \approx 2.38 \%$ probability that the two Purple group members are both selected to receive the low $\{30 \%, 40 \$\}$ employment chances.

Under the two Gray $(\mathrm{G})$ treatments, the five subjects insured under the Partial scheme are those who enjoy the high employment chance of at least $50 \%$, i.e. have employment chances in the $\{50 \%, \ldots, 90 \%\}$ set. Notice that under the Gray Inequality (GI) treatment, the unequal distribution of employment chances implies that all the Green participants are automatically included in the Partial insurance, even though the participants are not explicitly informed about this and are unaware of the existence of the two color types. ${ }^{9}$ On the other hand, under the Gray Equality (GE) treatment the Partial insurance may include both Green and Purple participants, since both types can draw a low employment chance. Finally, in both Gray treatments there is a monetary incentive for the subjects with at least 50\% employment chance to vote for Partial insurance and thus to exclude the 'unlucky' participants with lower employment chances.

\subsubsection{Voting Mechanism}

An interesting aspect of immigration in modern countries is that typically only the native population is allowed to vote, but that its electoral choices directly influence the welfare of non-voting immigrants. In our study this is represented by the following voting scheme: the choice of insurance scheme in a particular period in a particular group is based on the votes of the five Green subjects from that group, while the two Purple participants only indicate their preferences and have no effect on the final outcome of the elections. In other words, only the Green participants decide on the inclusivity

\footnotetext{
${ }^{9}$ However, all subjects do know their own set of possible employment chances and are thus aware whether they themselves will always be included in, or always be excluded from, the partial insurance scheme.
} 
of the insurance, even though Purple participants are potential contributors and beneficiaries.

The specific voting procedure is as follows. First, all participants are asked for their most preferred choice from the three options above. Next, all participants are asked to select their second preferred choice from the two remaining options, in case their most preferred choice is not implemented.

For the Purple subjects, the voting decision is framed as a preference questionnaire. These subjects however are informed that their payoff depends on their group's insurance scheme. ${ }^{10}$

For the Green subjects, the choice is framed explicitly as a voting decision. Regardless of the treatment, these participants are told that the "most popular" option will be implemented. Under the two Gray treatments, the Green voters do not realize that only five out of seven members of their group vote and that the two Purple ones are irrelevant. ${ }^{11}$ On the other hand, in the two Colorful treatments the Green subjects are informed that only their five votes matter and that Purple subjects do not possess any voting rights.

The choice of the insurance scheme is based on the standard ranked voting mechanism. Once the votes are cast, the choice with majority of Green votes (i.e. with three, four or five votes) wins. In the case that the most preferred choices of the Green subjects in a group do not provide a clear victory and two choices tie with two votes each: ${ }^{12}$ the tie is broken based on the second preferred choice of the fifth Green voter who cast their vote for the least popular option. For example, if Green subjects 1 and 2 voted for Full, subjects 3 and 4 for Partial, and the fifth subject was the only one to vote for No, their second preference will break the tie.

\subsubsection{Income inequality and group identity: Treatment Summary}

Our experiment is designed to disentangle the effect of income inequality from group identity, in the context of the labor market and unemployment insurance schemes. We highlight this by the following summary of the treatments.

- Gray Equality (GE) treatment:

In this treatment, all participants have the same expected employment chances and are unaware of the split into two groups. Moreover, the Partial insurance scheme covers all lucky

\footnotetext{
${ }^{10}$ We decided to assign the role of Purple group members to actual subjects, who are visible to the Green subjects, instead of computerized bots, in order to ensure that we elicit actual other-regarding preferences.

${ }^{11}$ Note that we are careful not to deceive subjects. We do tell Green subjects that they themselves are always allowed to vote and that this is not the case for all members of their group. However, they are not told how many - and which - group members are not allowed to vote. Furthermore, they are not told whether some subjects are always excluded from voting. Hence, Green subjects are unaware of a presence of an "immigrant" minority that is never allowed to vote, which is the main purpose of having this treatment dimension.

${ }^{12}$ Note that with three options and five voters, the only possible results are 5:0:0, 4:1:0, 3:2:0, 3:1:1 and 2:2:1, where only the last one is a tie without a clear majority.
} 
participants with an employment chance of at least $50 \%$. This is the only treatment where Purple participants may also benefit from the Partial insurance scheme and do not have any disadvantage other than not being able to vote.

- Gray Inequality (GI) treatment:

There now is an unequal distribution of skills or labor market discrimination, expressed as uneven employment chances. Each individual Green participant knows that they always have an employment chance of at least $50 \%$ and is better-off than some others. Moreover, participants know that the Partial insurance scheme will only cover those with employment chances of at least $50 \%$. The Green participants hence know that they always will be covered by this insurance scheme. However, since participants are not told that subjects are divided into two color types, the group identity does not play a role in the Green subjects' decisions.

- Colorful Equality (CE) treatment:

Subjects are informed about the existence of the two color types and know that the Partial insurance only covers the Green subjects, while excluding the Purple participants. However, in this treatment, both color types have the same ex-ante employment probabilities. The Partial and Full insurance schemes, hence, result in exactly the same expected payoffs for Green participants. Therefore, a preference for the Partial over the Full insurance scheme would be driven solely by group identity and other-regarding preferences.

- Colorful Inequality (CI) treatment: The final treatment combines group identity as in the CE treatment with inequality as in the GI Treatment.

\subsubsection{Payoffs}

Subject payoffs are based on the implemented insurance scheme, their employment status and their type: either Green or Purple. Detailed tables of the payoffs for all treatments and for the Green and Purple participants can be found in Appendix 3.7.

The baseline payment for an employed subject in any period is 400 points, while it is 80 points for an unemployed participant. Notice that these payments are the same for the Green and the Purple subjects, and thus that, in the absence of insurance, an ex-ante potential income inequality in the two Inequality treatments is caused only by the uneven distribution of the employment chances. The above payoffs are the final payoffs in the case that No insurance scheme is implemented, and also for all participants that are not covered by the Partial insurance in the case that scheme is implemented.

If there is an insurance scheme in place, unemployed and covered subjects receive an unemployment benefit which is added to the baseline unemployed payment of 80 . Employed and covered participants, on the other hand, need to pay a tax, which is subtracted from their baseline payment of 400 . This 
tax is chosen such that the implemented insurance scheme is ex ante self-financing in expected terms, i.e. the ex ante expected sum of the benefits is equal to that of of the contributions. ${ }^{13}$

In the case of Full insurance in all treatments, as well as in the case of Partial insurance under the $\mathrm{CE}$ treatment, the unemployment benefits are equal to 150 , so that the payoff of a covered unemployed subject becomes 230 points. This is financed by taxes equal to 100 points per covered employed participant, whose payoff becomes 300 points.

In the GI, GE and CI treatments, only subjects with a high employment chance of $50 \%$ or more are covered by Partial insurance scheme. The unemployment benefits under Partial insurance hence are a bit higher here (175 points), while taxes needed to finance them are lower ( 75 points). Final payoffs of covered employed (unemployed) subjects hence become 325 (225) points.

\subsubsection{Group formation game}

As mentioned above, the Green subjects under the two Gray treatments were unaware that they are divided into two color types. However, in the two Colorful treatments subjects were informed about the Purple participants, who cannot vote but can still be part of the insurance scheme. We wanted to guarantee a possibility of the Green players to form a group identity without resorting to politically charged framing about immigrants, and therefore asked them to play a simple game at the very start of their two sessions.

Our Bamberg lab consist of two adjoined rooms. At the very beginning of both the $\mathrm{CI}$ and $\mathrm{CE}$ treatment sessions, once the laboratory was closed and participant list was checked, we asked the 20 Green subjects to stay in the first, rather crammed, room and the 8 Purple participants to move into and wait in the second room. Then, the Green players were given 2 minutes to sort themselves alphabetically based on their first names, which we then checked with the list by asking them to shout their name aloud. Interestingly, both groups performed this task quickly and without a single mistake, and were rewarded with the promised 1 EURO additional reward at the end of the session. Once the game finished, all subjects were seated next to their computer stations, where the Purple players were visibly marked with purple sheets of paper above their computers.

\subsection{Testable Hypotheses and Experimental Procedures}

Below we will present and motivate our testable hypotheses in Section 3.4.2 Before we do that, we introduce the way in which we will operationalize the concept of polarization which plays an important role in our hypotheses (Section 3.4.1). We will end this section with an overview of the experimental

\footnotetext{
${ }^{13}$ In a repeated setting, this implies that the insurance scheme is self-financing in the dynamic sense, i.e. a period of high unemployment will be almost surely offset by a period of low unemployment.
} 
procedures (Section 3.4.3).

\subsubsection{Polarization}

As explained in the introduction, a major theme of our paper is political polarization. We will operationalize this phenomenon in its simplest form: as a substantial heterogeneity of votes. A particularly interesting version of such a polarization would be a scenario where our subjects would increasingly split between Full and No. In three out of four treatments, the Partial insurance is at least as beneficial as Full for the Green subjects, thus it seems reasonable that subjects with some degree of risk aversion would be naturally attracted to it. If, however, we find that in any of these treatments the Full and the No scheme gain in popularity, this would suggest that voters react in a heterogeneous fashion: some become more inclusive towards the Purple participants, while others more exclusive towards all participants. Such a reaction is likely to be driven by different forms of other-regarding preferences. In practice, the native population tends to be much larger than the migrant one, hence such a polarization has a potentially much greater impact on the labor market as a whole.

We also want to emphasize that none of these schemes on its own has to be interpreted as "radical", hence our definition of polarization pertains less to extreme ideologies (which is what that term points to in natural language) and more towards extreme differences between the voters.

\subsubsection{Testable Hypotheses}

The difference between Equality (E) and Inequality (I) treatments highlights the effect of ex-ante inequality of opportunities and ex-post income inequality in a society. In particular, the Gray Equality (GE) serves as a benchmark to study the underlying preferences of our subjects on unemployment insurance. The Gray Inequality (GI) treatment will reveal the pure effect of income inequality on willingness for redistribution. At the same time, the two Colorful $(\mathrm{C})$ treatments will reveal the effect of the division between the native Green population and the Purple immigrant one, i.e. the effect of group identity on social welfare choices.

We will therefore focus on the following three hypotheses:

Hypothesis 1: Voting decisions respond to incentives regarding individual (expected) payoffs:

(a) Green subjects with relatively high employment chances will be less willing to support more redistributive insurance schemes, regardless of the treatment;

(b) Participants that would be covered by both the Full and the Partial insurance schemes have a preference for Partial when this insurance scheme gives them a strictly higher payoff than Full. 
This Hypothesis is the least controversial from the point of view of mainstream economic literature. Hypothesis H1(a) implies that, regardless of the treatment, Green subjects will more likely switch away from Full and Partial towards No when they draw higher individual employment chances. Regarding Hypothesis H1(b), recall that the Partial scheme gives a strictly higher payoff than Full (for those that are covered by it) in treatments GE, GI and CI, but not in CE. This Hypothesis therefore predicts a clear preference for Partial insurance over Full insurance in the former 3 treatments, but not in the latter.

Hypothesis 2: The Green subjects' support for redistributive insurance schemes depends ceteris paribus:

(a) negatively on having more equal ex-ante employment and thus income chances (Full and Partial being less popular relative to No in CE vs. CI and GE vs. GI);

(b) negatively on the revelation of the group identities when redistribution towards Purple subjects is concerned (Full being less popular than Partial and No in CE vs. GE and CI vs. GI).

This is our main Hypothesis. It implies the existence of two important factors in subjects' preferences. Firstly (H2a), under the two income inequality treatments CI and GI, the Green subjects enjoy a visibly better economic status compared to the Purple subjects (even if they do not observe the colors under the GI treatment), which may result in them having a higher degree of acceptance of the economic and political hierarchies preordained by these treatments, and thus be more willing to exhibit a pro-social behavior (i.e. voting for either Full or Partial insurance with higher frequency). Secondly (H2b), the Green subjects framed with in-group identity may react negatively towards the Purple out-group, which would entail lower voting frequency for the Full insurance under the two Colorful treatments.

Hypothesis 3: The polarization of the Green subjects' attitudes towards redistributive insurance schemes will depend:

(a) positively on having more equal ex-ante employment and thus income chances;

(b) positively on the revelation of the group identities.

This Hypothesis predicts that both (a) the two Equality treatments as opposed to the Inequality ones, as well as (b) the two Colorful treatments in comparison with the Gray ones, should have a higher dispersion of votes. In particular, GI and CE should be the least and the most polarized treatments respectively.

The three Hypotheses are closely related to the literature which we discussed in detail in Section 3.2 Im sum, Hypothesis 1 refers to the vast body of research that investigates how monetary incentives influence performance, or - as in our case - preferences over redistribution. Even though, as pointed 
out by Awasthi and Pratt (1990, p. 798) "the effects of monetary incentives on performance cannot be generalized across different settings", we nonetheless support the idea that our Green subjects will, at least to some extent, try to optimize their expected payoff balanced with some risk aversion penalty. This motivates the two parts of this Hypothesis, as described above.

Hypotheses 2 and Hypotheses 3 are related to the link between immigration/ethnic diversity, preferences for redistribution and other-regarding preferences in general. While Hypothesis 2 illustrates two possible channels that may affect the Green subjects' acceptance of redistributive policies, Hypothesis 3 highlights the possibility that immigration combined with loss of the relative economic position by the native population may lead to an additional political backlash and polarization. In particular, hypotheses H2(a) and H3(a) are based on the main contribution by Campbell (1965) where competition for real resources (or - as in our case - competition in the labor market due to changes in the perceived economic prestige from Green subjects) may intensify intergroup conflicts. Hypothesis H2(a) postulates that changes in the economic perceived prestige of Green subjects may reduce their acceptance for more supportive redistribution policies (by switching towards No). Hypothesis H3(a) points to the possibility that changes in the economic perceived status of Green subjects may polarize their voting behavior, resulting in more heterogeneous voting patterns. Hypotheses H2(b) and H3(b) focus on the groups identity theory, proposed by Tafjel and Turner (1979), as a source of discrimination. Hypotheses H2(b) pertains to the direction in which the Green subjects' acceptance for more redistributive policies is affected by the revelation of the two different groups, the effect which we expect to be negative. Moreover, Hypothesis H3(b) illustrates how voting behavior of the Green subjects is affected by the presence of Purple migrant population, which again we expect to result in more polarization society. As pointed out by Alesina et al. (2019), Senik et al. (2009) and among other, there is a negative association between immigration and more support for redistribution from the native population (in our case Green subjects) towards immigrants (in our case Purple subjects). Similarly, as indicated by Majlesi et al. (2020) and Colantone and Stanig (2018), polarization increases when foreign competition increases in local labor markets.

\subsubsection{Experimental procedures}

Our experiment was conducted in the Bamberg Laboratory for Experimental Research (BLER) at the University of Bamberg throughout the summer term 2019. We used the software oTree (Chen et al. 2016) to program the experimental GUI for the subjects. Most of the participants were students from the University of Bamberg. We run one session of 28 subjects per each treatment, with four sessions and 112 participants in total. The experiment was conducted in German language (including instructions, GUI and oversight). The experimental instructions for all treatments (translated to English) can be found in Appendix 3.7 .

All subjects were allowed to participate in one session only. Each treatment session lasted approx- 
imately one hour and 15 minutes. The exchange rate was set to 750 tokens (experimental points) for 1 Euro. Moreover, in the Colorful treatments, Green participants had the possibility to earn $€ 1$ with their initial group formation game mentioned above. If they succeeded in that game, $€ 1$ was added to the final payments of all participants in the session. Furthermore, each participant was paid $\mathrm{a} € 4$ show-up fee. Table 3.2 shows the number of sessions and treatments, and the number of Green and Purple subjects and periods in each treatment. Notice that the GI treatment session lasted for 20 periods, while all the remaining for $25 .{ }^{14}$ In total, we collected 2660 observations, which consists of 1900 observations for the Green subjects and 760 observations for the Purple subjects.

Table 3.2: Features of experimental sessions

\begin{tabular}{c|c|c|c|c}
\hline \hline Treatment & Sessions & Subjects & Periods & Total Observations \\
\hline \multirow{2}{*}{ GI } & 1 & 20 & 20 & 400 \\
\cline { 2 - 5 } & 1 & 8 & 20 & 160 \\
\hline \multirow{2}{*}{ GE } & 1 & 20 & 25 & 500 \\
\cline { 2 - 5 } & 1 & 8 & 25 & 200 \\
\hline \multirow{2}{*}{ CE } & 1 & 20 & 25 & 500 \\
\cline { 2 - 5 } & 1 & 8 & 25 & 200 \\
\hline \multirow{2}{*}{ CI } & 1 & $\mathbf{2 0}$ & 25 & 500 \\
\cline { 2 - 5 } & 1 & 8 & 25 & 200 \\
\hline Total & & & & 2660 \\
\hline \hline
\end{tabular}

Each session had the following timing. Firstly, in the CI and CE sessions we ran the group formation game as discussed in Section 3.3.6. Next, subjects were seated at their computer stations and given time to read the instructions and answer control questions (see Appendix 3.7). Once we made sure that the game setup was clear, the proper part of the experiment started. It usually lasted around 50 minutes, after which the subjects answered a post-experiment survey which contained questions about demographical characteristics, risk attitudes, and strategies used during the experiment. Information about the payoffs, experimental instructions, and survey are included in Appendices $3.7 \quad 3.7$ and 3.7

\subsection{Experimental Results}

In our analysis we will focus on the Green subjects, since as the "native" population they had a monopoly on voting and thus a clear incentive to vote as to secure the implementation of their desired election results. As we mentioned before, the Purple participants, who represent the immigrants, do

\footnotetext{
${ }^{14}$ The first session served as a successful pilot, after which we realized that the experiment can run for 25 periods and still fit in the allocated time.
} 
not enjoy the voting rights, but only indicate their preferences without any influence on the voting outcome. As is standard in the economic experimental literature, we will not analyze in detail these unincentivized choices. ${ }^{15}$

In Section 3.5.1 we first present the aggregate experimental data and consider how the behavior of our subjects generally differed across treatments and across sampled employment chances. We will also shed some light on the validity of Hypotheses 1 and 2. Next, we turn to a more in-depth analysis of the individual decisions in Section 3.5.2, where we confirm these initial results. In Section 3.5.3 we will move the discussion to the issue of polarization and explicitly address Hypothesis 3. Following this, in Sections 3.5.4 we gather our findings on the attitudes of in-group Greens towards out-group Purples and provide an interpretation. Finally, we present the results related to our post-experimental survey in Section 3.5.5.

\subsubsection{Overview}

Table 3.3 shows the frequencies of winning regimes, as well as the frequencies of votes, for the four treatments. Table 3.4 reports the results for pairwise $t$-tests for whether these frequencies are significantly different for each strategy and each pair of treatments. The Partial insurance is by far the most preferred policy choice under the GI treatment with $83 \%$ of groups and $61 \%$ of subjects voting for it over the other two options. This result is not surprising, since under this treatment the Green subjects have a clear monetary incentive to chose the Partial insurance over the Full one. This is because both schemes cover them, but the Partial one is cheaper and provides higher benefits. We can therefore conclude that in the absence of group identity, participants seem to clearly respond to the individual monetary incentive and prefer an insurance scheme that is better for them. This is in line with Hypothesis 1(b).

An interesting additional observation is the clearly lower frequency of the actual votes on the Partial compared to times this scheme has won a majority. The second most popular choice was No with $23 \%$, and as will be explained later, this represents Green subjects who had high enough employment chances to risk having no insurance at all. This requires some luck, however, and in practice such subjects tended to be a minority within any given group, and thus they rarely could impose their preferred choice.

\section{Employment chances}

Next, we consider how the voting decisions depended on the employment chances that participants were facing. In Figure 3.2, we plot, for each treatment, how the the fractions of votes for Full (blue

\footnotetext{
${ }^{15}$ We leave for further research whether there exists a systematic difference between these unincentivized choices and the incentivized subjects' voting decisions.
} 
Table 3.3: Frequencies of winning strategies and overall votes (only Green participants' decisions).

\begin{tabular}{c|c|c|c|c|c|c}
\hline \multirow{2}{*}{ Treatment } & \multicolumn{3}{|c}{ \% of regimes } & \multicolumn{3}{c}{ \% of votes } \\
\cline { 2 - 7 } & Full & Partial & No & Full & Partial & No \\
\hline GI & 7 & 83 & 10 & 16 & 61 & 23 \\
\hline GE & 17 & 52 & 31 & 28 & 39 & 33 \\
\hline CI & 38 & 52 & 10 & 39 & 40 & 21 \\
\hline CE & 40 & 30 & 30 & 35 & 29 & 36 \\
\hline \hline
\end{tabular}

Table 3.4: T-test statistics for differences in regimes and votes between each pair of treatments. *,** and $* * *$ denote $10 \%, 5 \%$ and $1 \%$ significance level respectively.

\begin{tabular}{l|c|c|c|c|c|c}
\hline \hline & \multicolumn{3}{|c|}{ Regimes } & \multicolumn{3}{c}{ Votes } \\
\hline Treatments & Full & Partial & No & Full & Partial & No \\
\hline GI vs. GE & $-1.9794^{* *}$ & $4.6251^{* * *}$ & $-3.6557^{* * *}$ & $-4.65^{* * *}$ & $6.7801^{* * *}$ & $-3.2031^{* * *}$ \\
\hline GI vs. CE & $-5.6555^{* * *}$ & $8.3547^{* * *}$ & $-3.5026^{* * *}$ & $-6.7234^{* * *}$ & $10.075^{* * *}$ & $-4.3564^{* * *}$ \\
\hline GI vs. CI & $-5.3435^{* * *}$ & $4.6251^{* * *}$ & 0 & $-8.1727^{* * *}$ & $6.2719^{* * *}$ & 0.9519 \\
\hline GE vs. CE & $-3.707^{* * *}$ & $3.2289^{* * *}$ & 0.15282 & $-2.113^{* *}$ & $3.2878^{* * *}$ & -1.1963 \\
\hline GE vs. CI & $-3.4044^{* * *}$ & 0 & $3.7903^{* * *}$ & $-3.5646^{* * *}$ & -0.51683 & $4.398^{* * *}$ \\
\hline CE vs. CI & 0.28855 & $-3.2289^{* * *}$ & $3.6332^{* * *}$ & -1.4426 & $-3.8103^{* * *}$ & $5.6153^{* * *}$ \\
\hline \hline
\end{tabular}

squares), Partial (red circles) and No (green triangles) vary with the employment chances (horizontal axis). The four panels correspond to the four different treatments.

In all treatments, the green curves (frequencies of No votes) are clearly upward sloping for higher employment chances and close to zero for low employment chances. The other two curves (frequencies of Full and Partial votes) are generally downward sloping along the employment chances axis. The clear exception is the red curve (frequency of Partial votes) in panel 3.2c, which shows a very low frequency of Partial votes for subjects that had employment chances of $30 \%$ and $40 \%$ in the GE treatment. This is not surprising, as, in that treatment, Green subjects with employment chances below $50 \%$ are not covered by the Partial insurance scheme and hence prefer the Full scheme.

Generally, we can conclude that a participant, when confronted with a low employment chance, preferred to have an insurance scheme in place (either Full or Partial) over having no insurance at all. However, the higher the employment chance, the more participants decided that they did not need any insurance and voted accordingly in that round (for No). This is a clear indication that participants, at least to some extent, were in each round acting in a manner that maximized their expected payoff. Moreover, this finding is a first indication of support for Hypothesis 1(a). 


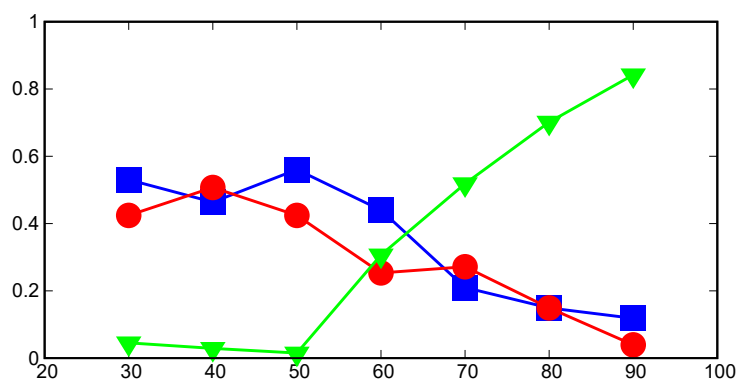

(a) $\mathbf{C E}$

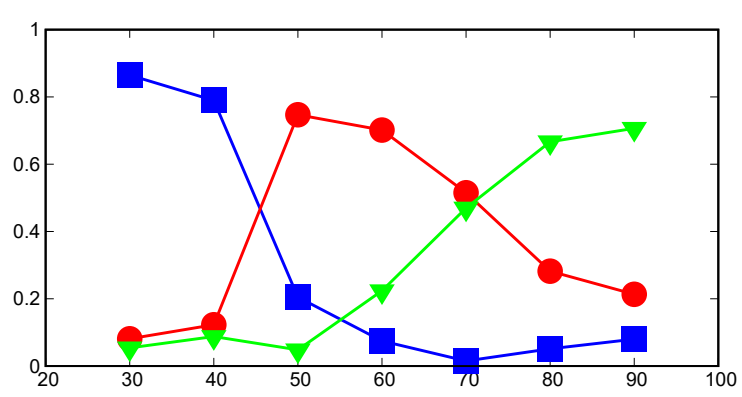

(c) GE

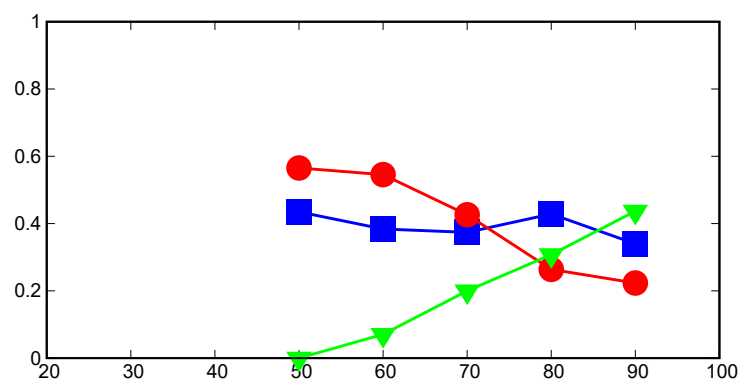

(b) CI

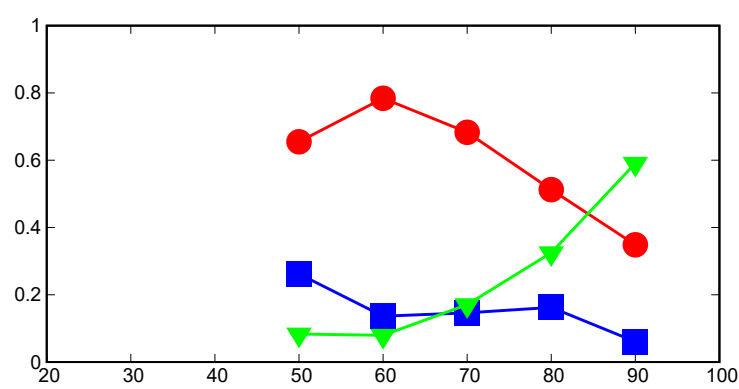

(d) $\mathbf{G I}$

Figure 3.2: Realized voting frequencies for Full (blue squares), Partial (red circles) and No (green triangles) insurance schemes, as a function of employment chance $\{30, \ldots, 90\}$, in each treatment.

For comparison, we present the risk neutral expected payoff maximizing choices for each treatment and for all employment chances in Table 3.5. If participants were risk neutral and only cared about their own payoffs, they should chose an insurance scheme for lower employment chances and then switch to No insurance when they have employment chances above 0.7 or 0.8 . The increases in the fraction of No votes from employment chances of 0.6 onward is qualitatively in line with this theoretical prediction. On the other hand, the fact that green curve is gradually upward sloping and does not jump from 0 to 1 indicates that there might be heterogeneity in risk aversion and other-regarding preferences among the pool of our participants.

Another observation that can be made from Figure 3.2 is that subjects with at least $50 \%$ employment chance clearly preferred Partial insurance over Full insurance, just as in the GI treatment. This is a clear indication that in the Gray treatments subjects that are covered by partial insurance prefer this option over Full insurance. Since Partial insurance gives a strictly higher individual payoff than Full in these two treatments, this confirms Hypothesis 1(b) as far as the Gray treatments are concerned. On the other hand, in the CI treatment, the revelation of color seems to considerably affect subjects' preference regarding Partial vs. Full insurance, so that no direct confirmation of Hypothesis 1(b) can be found here. We will turn to this group identity effect below in Section 3.5.1. 
Table 3.5: Risk neutral payoff maximizing choices for each treatment for different employment chances. Two choices in the same entry indicates indifference.

\begin{tabular}{ccccc}
\hline \hline Employment chance & GI & GE & CI & CE \\
\hline $30 \%$ & - & Full & - & Partial/Full \\
$40 \%$ & - & Full & - & Partial/Full \\
$50 \%$ & Partial & Partial & Partial & Partial/Full \\
$60 \%$ & Partial & Partial & Partial & Partial/Full/No \\
$70 \%$ & Partial/No & Partial/No & Partial/No & No \\
$80 \%$ & No & No & No & No \\
$90 \%$ & No & No & No & No \\
\hline \hline
\end{tabular}

\section{Income equality effect}

What is the effect of income equality on the above results? Comparing the two Gray treatments in Tables 3.3 and 3.4, we observe a statistically significant shift of votes away from Partial towards both Full and No alternatives when moving from GI to GE. Partial insurance was still implemented in half of the groups in GE. However, majority voting for the Full and No insurances were now more than twice and thrice as likely, respectively. Comparing the blue curves (votes for Full scheme) in panels 3.2c and 3.2d of Figure 3.2, it can be seen that the higher overall popularity of Full in GE compared to GI is purely driven by votes of participants with low employment chances. As discussed above, in the GE treatment, the only way in which Green participants can be insured when they have low employment chances is when Full insurance is implemented. For votes of participants with employment chances of $50 \%$ and higher, Full insurance, if anything, is less popular under GE than under GI.

The relative popularity of the No insurance, on the other hand, is at a first glance more difficult to understand. Under the GI treatment, many Green individuals with high enough employment chances decided that the insurance against unlikely unemployment is not worth the fixed cost. Under the GE treatment, sampling a high employment chance is in fact less likely, hence one would expect a lower frequency of votes for No. The fact that we observe the opposite suggests that, for a given employment chance, participants are less willing to contribute to redistribution in the Equality treatment than in the treatment where they are more privileged by their belonging to the in-group. A similar picture arises in Figure 3.2, where the green curve in panel 3.2d lies strictly lower than the green curve in panel $3.2 \mathrm{c}$.

Next we turn our attention to the role of inequality in the Colorful treatments. The effect of Inequality and Equality on the popularity of the No insurance is also evident when the two Colorful 
treatments are inspected in detail. Comparing $\mathrm{CI}$ and $\mathrm{CE}$, income equality again resulted in votes shifting away from the Partial insurance towards the No insurance, which became the most popular choice. This did not translate however into the No option being the most frequent electoral outcome (in fact Full was), suggesting much more polarized voting pattern than in the three other treatments, where Partial was both the most popular choice and the most frequent electoral outcome. Moreover, in Figure 3.2 the green line (frequency of No votes) for the CI treatment is again strictly lower than in the CE treatment. Furthermore, the difference seems to be even more pronounced than for the Gray treatments. We discuss a possible explanation for this in Section 3.5.4 which points towards a certain form of polarization. All in all, the increased popularity of No in both Equality treatments relative to their Inequality counterparts points towards a decrease in Green subject's support for redistributive insurance schemes, in line with Hypothesis 2(a).

On the other hand, the additional shift from Partial to Full that we observed in the Gray treatments does not arise when moving from CI to CE. This is because in both CE and CI, Green participants are always covered by the Partial insurance. They, therefore, never have an incentive, from the perspective of their own payoffs, to prefer Full insurance over Partial insurance, as they had in the GE treatment.

\section{Group identity effect}

Next, we consider the effects of group identity in more detail. Let us focus firstly on the difference between the GI and CI treatments. To our surprise, we do not observe the Partial scheme to become relatively more popular under the CI treatment. In fact the opposite happens: there is a significant shift of votes from Partial towards Full insurance, which also makes the latter strategy a much more likely electoral outcome (38\% in contrast to $7 \%$, see Table 3.3 ). On the other hand, in these two treatments there are no significant differences in popularity of the No insurance.

It seems that once the subjects were made aware of the relative economic and voting disadvantage of the Purple population, some Green participants took pity on these underprivileged subjects and chose the Full insurance instead of the Partial scheme. This suggests some "positive" other-regarding preferences, since Partial insurance is still individually more profitable for the Green subjects than the Full scheme. This contradicts Hypothesis 2(b).

Similar changes can be observed when comparing the GE and CE treatments. When looking at the aggregate votes in Table 3.3, the shift from Partial to Full is less pronounced here. However, as can be seen in Figure 3.2, this is driven by low employment probabilities only, where Green participants are not covered by the Partial insurance. When one considers only votes by Green participants with employment chances of $50 \%$ and higher in these two treatments, there is a clear shift from Partial (red) to Full (blue). This again suggests that Hypothesis 2(b) is false. 


\section{Summary of aggregate results}

To sum up, the aggregate results of our experiment suggest the following mechanisms behind the decisions of our subjects:

- Participants that faced low employment chances favored an insurance scheme (Full or Partial) in all treatments, whereas participants facing higher employment chances increasingly voted for No insurance.

- In treatments where the Partial insurance scheme was cheaper and provided higher unemployment benefits than the Full one, the majority preferred the Partial insurance scheme. In the treatment where this was not the case $(\mathrm{CE})$, they did not.

- If Green participants with low employment chances are not covered by partial insurance (as in the GE treatment), they prefer Full over Partial when sampling low employment chances.

- Equalizing employment chances and thus ex ante income results in an increased popularity for No insurance. ${ }^{16}$

- Participants reacted to type revelation mostly with compassion and voted more often for the Full scheme.

In other words, subjects seem to have composite preferences, where they consider their expected monetary outcome, but also their relative ex ante income level They react kindly to the out-group subjects, but mostly if they still enjoy a higher economic status (expressed by the income inequality treatment). All in all, this provides empirical support for Hypotheses 1(a), 1(b), and 2(a). Hypothesis 2(b) seems not to be corroborated by the aggregate results.

\subsubsection{Individual unemployment insurance preferences}

To formally examine how the group identity and income inequality affect subjects' preferences, we use a standard multinomial logistic regression model with individual random effects for all choices taken by all Green subjects, across all the groups and periods. As explained earlier, our subjects had in fact a choice of six possible voting strategies: firstly, they had to pick one out of three insurance scheme as their most preferred one, and then one out of the two remaining as their second most preferred one. We will use the following notation: when a Green subjects chooses for instance Full_Partial strategy, they pick Full as their most preferred outcome and Partial as the second one, which leaves No being the least preferred outcome - and similarly for the five other possible strategies. ${ }^{17}$

\footnotetext{
${ }^{16}$ The implications of this result for the issue of polarization will be discussed in Section 3.5.3

${ }^{17}$ We also re-estimated the model with only the first choice as the explained variable, see the results in Appendix 3.7 These estimations generally yield similar results to those that are described in this Section, however, they are based on a smaller information set and thus less reliable.
} 
Table 3.6: First and second decisions with non-linear employment probabilities. No_Partial insurance, CE treatment and Ch5060 as base outcome

\begin{tabular}{|c|c|c|c|c|c|}
\hline Variables & 1.Full_No & 2.Full_Partial & 3.No_Full & 5.Partial_Full & 6.Partial_No \\
\hline Ch3040 & 0.377 & $2.020^{* * *}$ & -19.93 & $1.641^{* * *}$ & -1.603 \\
\hline Ch7080 & $-2.687^{* * *}$ & $-4.037^{* * *}$ & -0.590 & $-3.386^{* * *}$ & $-1.471^{* * *}$ \\
\hline Ch90 & $-4.171^{* * *}$ & $-6.078^{* * *}$ & $-1.568^{* * *}$ & $-5.301^{* * *}$ & $-2.951^{* * *}$ \\
\hline CI & 2.158 & 2.162 & -1.234 & $3.145^{* * *}$ & $1.971^{* * *}$ \\
\hline GE & -1.977 & -2.012 & $-2.830^{* *}$ & 0.0442 & 0.141 \\
\hline GI & -1.507 & -0.519 & $-4.023^{* * *}$ & 1.104 & $1.282^{* *}$ \\
\hline poorGE & $2.826^{* * *}$ & 0.638 & 21.42 & $-2.701^{* * *}$ & -0.636 \\
\hline employed_Freq & 0.396 & -1.447 & 0.532 & -1.169 & -0.477 \\
\hline insured_Freq & -1.715 & -0.214 & -0.166 & -0.561 & -0.699 \\
\hline full_Freq & 1.844 & 0.673 & -0.640 & 0.148 & 1.712 \\
\hline partial_Freq & 0.749 & 0.255 & -0.0535 & 0.445 & 1.214 \\
\hline employed_insured_Freq & -0.906 & 0.367 & -1.099 & 1.086 & 0.237 \\
\hline ind. RE & 1 & $1.167^{* * *}$ & $0.745^{* * *}$ & $0.633^{* * *}$ & $0.303^{* * *}$ \\
\hline Constant & 1.529 & $3.183^{* *}$ & $1.661^{*}$ & $2.829^{* * *}$ & 0.444 \\
\hline Observations & 1900 & & & & \\
\hline
\end{tabular}

In our estimations we consider the treatments (fixed per subject) and employment chances (which change from subject to subject and from period to period). We use the latter as a categorical variable, which we split into four categories and which potentially has a non-linear relationship with the preferences. The four considered categories are employment chances in four following subsets: $\{30 \%, 40 \%\}$, $\{50 \%, 60 \%\},\{70 \%, 80 \%\}$ and $\{90 \%\},{ }^{18}$ We also include an interaction dummy for Green subjects who under the GE treatment sampled "poor" employment chance (30\% or $40 \%$ ) in a given period $t$. This category captures the only possible scenario where the Green subjects are excluded from the Partial insurance scheme.

Finally, we control the results with additional five experiential factors. For each subject $i$ at each period $t$, we compute how many times they experienced: being employed (employed_Freq), insured (insured_Freq), being both employed and insured (employed_insured_Freq), being in a group that chose the Full scheme (full_Freq), and the Partial scheme (partial_Freq). For obvious reasons, these variables are transformed into frequencies. For instance, full_Freq ${ }_{i, 6}=0.6$ implies up to the beginning

\footnotetext{
${ }^{18}$ See Appendix 3.7 for estimations with the employment chance treated as a continuous variable. These estimations predicate a linear relationship with the strategy choices of our subjects and we felt this to be a potentially limiting assumption.
} 
of period 6 , subject $i$ has participated in 5 previous elections, in which the Full scheme won 3 times (ie. $60 \%$ of times).

The estimations (coefficients for the logistic map, together with their significance levels) are presented in Table 3.6. The benchmark case is a subject who chose No_Partial (what we consider the most exclusionary strategy), had an employment chance of either $50 \%$ or $60 \%$ (the lowest possible under the two inequality treatments) and participated in the CE treatment.

The following results are consistent and thus support the findings at the aggregate level discussed in Section 5.1. Firstly, the individual employment chances have a strong negative effect on how much the subjects are willing to support more inclusive insurance schemes. For instance, for the Green subjects with $90 \%$ employment chance, the typical preference ordering is given by No_Partial, No_Full, Partial_No, Full_No, Partial_Full and Full_Partial. On the other extreme, subjects with the lowest chances prefer Full_Partial and Partial_Full over the four other strategies. This further confirms Hypothesis 1(a).

Secondly, the treatments have the following direct effects on the subjects' decisions (see also Table 3.21 in Appendix 3.7 for the same estimation, but with GI as the benchmark treatment, for explicit significance tests):

- The two Inequality treatments push the subjects towards the Partial_No decision and away from the benchmark No_Partial. In addition, the CI treatment pushes the subjects towards the Partial_Full strategy in comparison with the CE treatment. This confirms Hypothesis 2(a).

- The two Gray treatments push the subjects away from the No_Full strategy towards the benchmark No_Partial. In addition the GI treatment pushes the subjects away from the Partial_Full and Full_No strategies in comparison with the CI treatment. As our earlier findings, this contradicts Hypothesis 2(b), which we therefore reject. ${ }^{19}$

- When moving from CE to GE treatment, "poor" Green subjects with employment chances of $30 \%$ and $40 \%$ (dummy variable PoorGE) switch away from Partial_Full and towards Full_No in almost an equal rate, which confirms that our subjects understood the available insurance schemes.

It should be noted that, again, Hypothesis 2(b) was not only rejected, in fact its opposite was confirmed: the revelation of group identity seems to increase the propensity of Green subjects to vote for inclusive insurance schemes. We will come back to this result in detail in Section 3.5.4 but first we want to discuss the issue of polarization in Section 3.5.3

Finally, one needs to emphasize that none of the experiential variable had a significant effect on the choices of our subjects. This result is consistent with the experimental design and proves

\footnotetext{
${ }^{19}$ The differences between CI and GI treatments are significant at $5 \%$ level. In addition, the CI increases propensity towards the Full_Partial strategy at $10 \%$ significance level, which we leave out from the discussion.
} 
that the subjects understood that their groups are re-sampled in every period, and thus that their previous experience is unlikely to be good predictor of the present. In other words, our experiment reveals, so to say, benchmark preferences of our subjects, without reputation effects, which we leave for future studies. To test the robustness of our estimations, we also re-estimate the same model without experiential variables, see Table 3.17 in Appendix 3.7

\subsubsection{Polarization}

To keep the discussion structured, we have so far focused on Hypotheses 1 and 2, while only occasionally referring to what the results imply for polarization. We now turn to the latter issue in detail and explicitly consider what we find regarding Hypothesis 3. In a nutshell, our results show that polarization depends on the interplay between three factors: income distribution, group identity, and also the realized employment chance in a particular period.

Recall from Section 3.4.1 that the generally most popular first choice of the Green subjects was the Partial insurance scheme. This implies that a treatment will reduce polarization, if it shifts the subjects - holding their employment chances constant - to the Partial_Full or Partial_No strategies. This is exactly what the estimated individual preferences point to for the case of the two Inequality treatments against their counterpart Equality treatments. This confirms Hypothesis 3(a).

The effect of the Colorful treatments is more complex, however. The CI treatment induces more of the Partial_Full strategy compared to the the GI treatment, but also more of the Full_No strategy. These two effects have opposite implications for the level of polarization and - judging from Table 3.3 and Figure 3.2 - the former effect is more relevant, so that the CI treatment is in fact more polarized. What is surprising about this polarization is that in fact it is beneficial for the migrant population. Apparently, Green subjects reacted with compassion to the presence of Purple ones and voted for more inclusive insurance Full scheme, compared to their baseline behavior. ${ }^{20}$

No such clear outcome can be observed between the CE and GE treatments, where the only baseline treatment effect is a shift between the No_Partial and No_Full strategies. However, the "poor" subjects under the GE treatment have in fact shifted from Partial_Full to Full_No. Given their employment chances, it is clear that these "poor" subjects prefer an unemployment insurance, and since only the Full scheme covers them, they vote for it over the Partial one. Their second choice indicates an "include me or include no one" attitude: if they cannot be insured, they prefer nobody else to be insured either.

Furthermore; under the GE treatments, the "middle class" subjects with $50 \%$ and $60 \%$ employment

\footnotetext{
${ }^{20}$ It would be interesting to study whether this result would also hold in a dynamic setting with reputation effects. Another issue is that our subject pool was mostly consisting of German students (as also confirmed by the survey data) who may be influenced by the particular German history. A cross-country study planned for future research will hopefully shed some light on the latter issue.
} 
chances voted mostly for the Partial scheme and only the lucky "upper class" subjects with the highest chances of $80 \%$ and $90 \%$ prefer No insurance, as clearly observable in Figure 3.2c. This polarization on the income lines indicates focus on maximizing individual utility.

Moving to the CE treatment, we observe that the "poor" and "middle class" subjects vote in a more homogeneous fashion, compared to the GE treatment (see Figure 3.2a). What is interesting is that if we were to pool these two groups, the average vote would in fact look very similar in the GE and CE treatments, with the "poor" and "middle" population splitting their votes roughly equally between the Full and Partial schemes, while the "rich" subjects switching to the No insurance. The difference between these two treatments lies therefore not in the average support for any insurance scheme, but in whether the Partial insurance is supported (CE treatment) or rejected (GE treatment) by the "poor" Green subjects, which is exactly the reason why in the estimated model reported in Table 3.6, the "PoorGE" variable is significant, while there are no significant overall treatment differences between the $\mathrm{CE}$ and GE treatments. In other words, an interesting difference between the $\mathrm{CE}$ and GE treatments lies in the structure, instead of the magnitude of polarization. Future studies should focus on studying this issue in detail, but our results suggest that it is at least possible that the presence of group identity encourages pro-social behavior at least among some of our subjects.

\subsubsection{Summary of the experimental findings on the attitude towards the migrant population}

Our experimental design focuses on two dimensions of how people vote for unemployment insurance: the group identity and income inequality. Our experimental results suggest that the former has a clear-cut (albeit counterintuitive) effect: when the Green subjects learn about the out-group Purple subjects, they in fact often vote for more inclusive insurance schemes to cover them. This effect is particularly clear in the two Inequality treatments, where the group identity causes a major shift of votes towards the Full insurance, despite it being more expensive for the Green subjects. However, the effect of income inequality is less clear and very much conditional on the employment chance that a Green subject has drawn. In particular, for low employment chances (30\% and 40\%), participants are trying to improve their situation through insurance schemes by which they are covered (Full in GE and Partial or Full in CE). Participants in the Equality treatments that face higher employment chances, on the other hand, voted less for Partial and more for No than in the Inequality treatments. How to interpret these results? We propose the following explanation: It is likely that the subjects in the experiment were considering two things: their (expected) income level, but also their relative economic and political position (?). In the two Inequality treatments, Green participants have an ex-ante privileged position and enjoy a visible economic as well as a political advantage over Purple subjects. This might have made some of them feel obliged to engage in more pro-social behavior and vote for a redistributive scheme, even in periods when they had relatively high employment chances 
and did not need an insurance scheme for themselves. This led to polarization of attitudes towards the immigrants.

On the other hand, in the Equality treatments, Green subjects did not enjoy such an economic advantage. They reacted to this with more selfish behavior. In particular, their votes reflected mostly their individual employment chances, and they were quicker to forgo any insurance in periods when they did not need one for themselves. Since, in any period, subjects in a group were likely to have heterogeneous employment chances, polarization based on income emerged as a natural byproduct of this process.

A further effect of the income equality comes through the difference between CE and GE treatments. In particular, even the relatively poor Green subjects in the CE treatment enjoyed an additional visible political advantage over the Purple ones, since they were able to vote to discriminate against that group, whereas the Partial scheme would not exclude them. We believe that this might have, to some extent, compensated them subjectively for their lower relative income under the $\mathrm{CE}$ treatment and thus might have induced a relatively more pro-social behavior. On the other hand, under the GE treatment, Green subjects do not enjoy this additional political advantage - in fact they understood that now they themselves could have been excluded from the Partial insurance scheme, which induced even more selfish behavior and polarization. In sum, polarization and discrimination seem to be thus driven by lower perceived economic and political status over the out-group population.

\subsubsection{Survey}

At the end of each experimental session, we asked the subjects to fill out a thorough questionnaire. The first part of the questions covered their demographic characteristics: gender, age, field of study, as well as nationality and whether they were second generation migrants. In the second part, the subjects had to assess the degree to which they agree or disagree with four statements about immigrants, such as "Germany has a problem with immigrants who do not integrate well". Finally, we tested the subjects' risk attitude with a hypothetical lottery game and asked them to elicit the degree to which they used current and previous employment chances in their decision making. 
Table 3.7: Survey (Full Frequency)

\begin{tabular}{|c|c|c|c|c|}
\hline & Model 1 & Model 2 & Model 3 & Model 4 \\
\hline \multirow[t]{2}{*}{ study3Dummy } & -0.116 & -0.0896 & -0.0711 & -0.0913 \\
\hline & $(-1.65)$ & $(-1.32)$ & $(-0.96)$ & $(-1.26)$ \\
\hline \multirow[t]{2}{*}{ countryDummy } & 0.0732 & 0.0827 & 0.0491 & 0.0503 \\
\hline & $(0.46)$ & $(0.54)$ & $(0.31)$ & $(0.34)$ \\
\hline \multirow[t]{2}{*}{ country_bornDummy } & -0.00716 & -0.0464 & -0.0369 & 0.0113 \\
\hline & $(-0.06)$ & $(-0.36)$ & $(-0.28)$ & $(0.09)$ \\
\hline \multirow[t]{2}{*}{ immigrant_bornDummy } & -0.0191 & -0.0530 & -0.0733 & -0.0421 \\
\hline & $(-0.22)$ & $(-0.62)$ & $(-0.82)$ & $(-0.50)$ \\
\hline \multirow[t]{2}{*}{ gender } & $-0.142^{* *}$ & $-0.138^{* *}$ & $-0.153^{* *}$ & $-0.120^{*}$ \\
\hline & $(-2.11)$ & $(-2.06)$ & $(-2.20)$ & $(-1.81)$ \\
\hline \multirow[t]{2}{*}{ age } & 0.00266 & 0.00350 & 0.00459 & 0.00416 \\
\hline & $(0.65)$ & $(0.87)$ & $(1.09)$ & $(1.06)$ \\
\hline \multirow[t]{2}{*}{ own_prob } & & $-0.0318^{* * *}$ & $-0.0287^{* *}$ & $-0.0390 * * *$ \\
\hline & & $(-2.87)$ & $(-2.40)$ & $(-3.33)$ \\
\hline \multirow[t]{2}{*}{ other_prob } & & -0.00569 & -0.00839 & -0.00886 \\
\hline & & $(-0.52)$ & $(-0.73)$ & $(-0.80)$ \\
\hline \multirow[t]{2}{*}{ past_prob } & & -0.00913 & -0.00724 & -0.00138 \\
\hline & & $(-0.68)$ & $(-0.52)$ & $(-0.10)$ \\
\hline \multirow[t]{2}{*}{ risk_ind } & & -0.00103 & -0.000896 & 0.00000239 \\
\hline & & $(-0.54)$ & $(-0.46)$ & $(0.00)$ \\
\hline \multirow[t]{2}{*}{ money1 } & & & -0.0190 & -0.0217 \\
\hline & & & $(-0.74)$ & $(-0.90)$ \\
\hline \multirow[t]{2}{*}{ money2 } & & & 0.0128 & 0.0109 \\
\hline & & & $(0.61)$ & $(0.55)$ \\
\hline \multirow[t]{2}{*}{ imm1 } & & & 0.00689 & 0.0120 \\
\hline & & & $(0.30)$ & $(0.56)$ \\
\hline \multirow[t]{2}{*}{ imm2 } & & & 0.0101 & 0.00645 \\
\hline & & & $(0.43)$ & $(0.29)$ \\
\hline \multirow[t]{2}{*}{ int1 } & & & -0.0137 & -0.0201 \\
\hline & & & $(-0.65)$ & $(-1.01)$ \\
\hline \multirow[t]{2}{*}{ int2 } & & & 0.00276 & -0.000165 \\
\hline & & & $(0.15)$ & $(-0.01)$ \\
\hline \multirow[t]{2}{*}{$\mathrm{CE}$} & & & & 0.0538 \\
\hline & & & & $(0.58)$ \\
\hline \multirow[t]{2}{*}{ CI } & & & & 0.0297 \\
\hline & & & & $(0.32)$ \\
\hline \multirow[t]{2}{*}{ GI } & & & & $-0.253^{* *}$ \\
\hline & & & & $(-2.55)$ \\
\hline Observations & 83 & 83 & 83 & 83 \\
\hline
\end{tabular}

$t$ statistics in parentheses

${ }^{*} p<0.10,{ }^{* *} p<0.05,{ }^{* * *} p<0.01$ 
We regressed all these variables on the frequency with which each subject voted for Full (as their first decision). Table 3.7 presents the results of this estimation for four different subsets of variables, including the treatment dummies. As can be observed, the majority of these variables have an insignificant effect. The only two clear regularities that we found is that (i) women tend to vote more often for Full than men and (ii) subjects who reported to consider their current employment chances voted slightly less often for Full. The latter observation reflects the findings from the previous part of this section, and suggest some degree of heterogeneity in other-regarding preferences among our subjects. On the other hand, since the demographic and political variables do not seem to play a large role in our experiment, we conclude that the labor market structure was much more important for our subjects than their preconceived attitudes towards migration, which is an important factor to consider for policy makers.

\subsection{Concluding Remarks}

The relationship between migration, income inequality and political polarization has been one of the main topics in the political discourse of the last years. Brexit, as well as popularity of nativist movements across Europe and in the USA are just some examples of a political backlash against recent waves of migration, related to the globalization and political instability in some regions of the world. It is important to study the reasons behind these backlashes, and two popular theories point towards (a) xenophobic attitudes towards culturally "other" migrants and (b) economic anxiety based on fears that relatively poorer migrants might become a burden on the already strained social security systems. Unfortunately, it is difficult to disentangle these two effects in the real world.

We studied this issue with a simple laboratory experiment. Subjects were split into two types, "natives" and "migrants", framed with colors as green and purple subjects. Each subject was repeatedly assigned at random to a group consisting of five green and two purple subjects, and sampled an employment chance. The green subjects were tasked with voting on alternative unemployment insurance schemes: they could choose one that covers nobody, everybody or only part of the population. We studied four treatments in a two-by-two design. In the first dimension, subjects were either unaware or aware of the distinction between the green and the purple players. In the second dimension, the native subjects had the same or a visibly more beneficial ex ante distribution of the employment chances.

Our results confirm some popular beliefs about the attitude towards social insurances and migration, but contradict other. Personal expected income was an important driving factor for our subjects, who tended to be the more selfish the higher the ex ante employment chance they sampled. The revelation of the group identities, contrary to a popular assumption (Dahlberg et al. 2012 and Tabellini 2020), has in fact induced compassion of the native population towards the migrants, in line with ?. 
However, subjects with a worse ex ante income distribution (meaning the employment chance distribution) were also more selfish regardless of the actual employment chance. These two effects caused more polarization as well, but with contradictory effects on the well-being of the migrant subjects. A cynical interpretation of these results is that, in contrast to what many nativist politicians claim, many of our subjects were in fact ready to accept immigrants and include them in social safety net, but only if the latter group would remain visibly poorer.

Further research should study this issue in detail, in particular the extent to which our results depend on the subject pool and Germany (with its peculiar recent history) being their country of residence. Another important issue, which was beyond the scope of interest of our research is the role of reputation and "lock-in" dynamics in groups with constant subject composition. We believe that further research on these issues may lead to further important insights. 
3.7 Appendix to Chapter 3 


\section{Payoffs Tables}

\section{Gray Equality for Green and Purple participants}

Table 3.8: Payoffs for Green and Purple participants in the GE treatment

\begin{tabular}{|c|c|c|c|c|}
\hline & \multicolumn{2}{|c|}{ Employment } & \multicolumn{2}{|c|}{ Unemployment } \\
\hline \multirow{3}{*}{ Full Insurance } & Points & 400 & Points & 80 \\
\hline & Tax & -100 & Subside & +150 \\
\hline & Net & 300 & Net & 230 \\
\hline \multirow{3}{*}{$\begin{array}{l}\text { Partial Insurance with high } \\
\text { employment probabilities }\end{array}$} & Points & 400 & Points & 80 \\
\hline & $\operatorname{Tax}$ & -75 & Subside & +175 \\
\hline & Net & 325 & Net & 255 \\
\hline \multirow{3}{*}{$\begin{array}{l}\text { Partial Insurance with low } \\
\text { employment probabilities }\end{array}$} & Points & 400 & Points & 80 \\
\hline & Tax & 0 & Subside & 0 \\
\hline & Net & 400 & Net & 80 \\
\hline \multirow{3}{*}{ No insurance } & Points & 400 & Points & 80 \\
\hline & Tax & 0 & Subside & 0 \\
\hline & Net & 400 & Net & 80 \\
\hline
\end{tabular}




\section{Gray Inequality for Green participants}

Table 3.9: Payoffs for Green participants in the GI treatment

\begin{tabular}{|l|c|c|c|c|}
\hline \multirow{4}{*}{ Full Insurance } & \multicolumn{2}{|c|}{ Employment } & \multicolumn{2}{c|}{ Unemployment } \\
\hline & Points & 400 & Points & 80 \\
& Tax & -100 & Subside & +150 \\
\cline { 2 - 5 } & Net & 300 & Net & 230 \\
\hline \hline \multirow{3}{*}{ Partial Insurance } & Tax & -75 & Subside & +175 \\
\cline { 2 - 5 } & Net & 325 & Net & 255 \\
\hline \hline \multirow{3}{*}{ No Insurance } & & & & 80 \\
\hline & Points & 400 & Points & 80 \\
\cline { 2 - 5 } & Tax & 0 & Subside & 0 \\
\cline { 2 - 5 } & Net & 400 & Net & 80 \\
\hline \hline
\end{tabular}

\section{Gray Inequality for Purple participants}

Table 3.10: Payoffs for Purple participants in the GI treatment

\begin{tabular}{|l|c|c|c|c|}
\hline \multirow{4}{*}{ Full Insurance } & \multicolumn{2}{|c|}{ Employment } & \multicolumn{2}{c|}{ Unemployment } \\
\hline & Points & 400 & Points & 80 \\
& Tax & -100 & Subside & +150 \\
\cline { 2 - 5 } & Nett & 300 & Nett & 230 \\
\hline \hline \multirow{3}{*}{ Partial Insurance } & Tax & 0 & Subside & 80 \\
\cline { 2 - 5 } & Nett & 400 & Nett & 80 \\
\hline \hline \multirow{3}{*}{ No Insurance } & & & & 80 \\
\hline & Points & 400 & Points & 80 \\
\cline { 2 - 5 } & Nett & 0 & Subside & 0 \\
\hline \hline
\end{tabular}


Colorful Inequality for Green participants

Table 3.11: Payoffs for Green participants in the CI treatment

\begin{tabular}{|l|c|c|c|c|}
\hline \multirow{4}{*}{ Full Insurance } & \multicolumn{2}{|c|}{ Employment } & \multicolumn{2}{c|}{ Unemployment } \\
\hline & Points & 400 & Points & 80 \\
& Tax & -100 & Subside & +150 \\
\cline { 2 - 5 } & Nett & 300 & Nett & 230 \\
\hline \hline \multirow{3}{*}{ Partial Insurance } & Tax & -75 & Subside & +175 \\
\cline { 2 - 5 } & Nett & 325 & Nett & 255 \\
\hline \hline \multirow{3}{*}{ No Insurance } & & & & 80 \\
\hline & Points & 400 & Points & 80 \\
\cline { 2 - 5 } & Tax & 0 & Subside & 0 \\
\cline { 2 - 5 } & Nett & 400 & Nett & 80 \\
\hline \hline
\end{tabular}

Colorful Inequality for Purple participants

Table 3.12: Payoffs for Purple participants in the CI treatment

\begin{tabular}{|l|c|c|c|c|}
\hline \multirow{4}{*}{ Full Insurance } & \multicolumn{2}{|c|}{ Employment } & \multicolumn{2}{c|}{ Unemployment } \\
\hline & Points & 400 & Points & 80 \\
& Tax & -100 & Subside & +150 \\
\cline { 2 - 5 } & Netto & 300 & Netto & 230 \\
\hline \hline \multirow{4}{*}{ Partial Insurance } & Tax & 0 & Subside & 0 \\
\cline { 2 - 5 } & Netto & 400 & Netto & 80 \\
\hline \hline \multirow{3}{*}{ No Insurance } & & 400 & Points & 80 \\
\hline & Points & 400 & Points & 80 \\
\cline { 2 - 5 } & Netto & 400 & Subside & 0 \\
\hline \hline
\end{tabular}


Colorful Equality for Green participants

Table 3.13: Payoffs for Green participants in the CE treatment

\begin{tabular}{|l|c|c|c|c|}
\hline \multirow{4}{*}{ Full Insurance } & \multicolumn{2}{|c|}{ Employment } & \multicolumn{2}{c|}{ Unemployment } \\
\hline & Points & 400 & Points & 80 \\
& Tax & -100 & Subside & +150 \\
\cline { 2 - 5 } & Netto & 300 & Netto & 230 \\
\hline \hline \multirow{4}{*}{ Partial Insurance } & Tax & -100 & Subside & +150 \\
\cline { 2 - 5 } & Netto & 300 & Netto & 230 \\
\hline \hline \multirow{3}{*}{ No Insurance } & & & & 80 \\
\hline & Points & 400 & Points & 80 \\
\cline { 2 - 5 } & Tax & 0 & Subside & 0 \\
\cline { 2 - 5 } & Netto & 400 & Netto & 80 \\
\hline \hline
\end{tabular}

Colorful Equality for Purple participants

Table 3.14: Payoffs for Purple participants in the CE treatment

\begin{tabular}{|l|c|c|c|c|}
\hline \multirow{4}{*}{ Full Insurance } & \multicolumn{2}{|c|}{ Employment } & \multicolumn{2}{c|}{ Unemployment } \\
\hline & Points & 400 & Points & 80 \\
& Tax & -100 & Subside & +150 \\
\cline { 2 - 5 } & Netto & 300 & Netto & 230 \\
\hline \hline \multirow{4}{*}{ Partial Insurance } & & & & \\
\cline { 2 - 5 } & Points & 400 & Points & 80 \\
& Netto & 400 & Subside & 0 \\
\hline \hline \multirow{3}{*}{ No Insurance } & & & & 80 \\
\hline & Points & 400 & Points & 80 \\
\cline { 2 - 5 } & Tax & 0 & Subside & 0 \\
\hline \hline
\end{tabular}




\section{Experimental Instructions}

\section{Gray Equality for Green and Purple participants}

This treatment does not inform about the existence of the two color types. All participants are unaware of the split into two groups. Moreover, Green and Purple participants always draw from the same probability employment distribution. The only difference between the instructions provided to Green and Purple participants in this and all following treatments is based on the rights to vote. While Green participants are asked to vote their most preferred choice (as indicated below in the instructions for Green participants), Purple participants are asked to indicate their most preferred outcome.

\section{Experimental instructions and general information}

Welcome to this experiment at the University of Bamberg! The experiment is anonymous, the data from your decisions will only be linked to your station ID, not to your name. You will be paid privately at the end, after all participants have finished the experiment. After the main part of the experiment and before the payment, you will be asked to fill out a short questionnaire.

During the experiment you are not allowed to use your mobile phone or any other communication devices. You are also not allowed to communicate with other participants. If you have a question at any time, please raise your hand and someone will come to your desk.

\section{Experimental economy}

The experiment will last for 25 periods. In each period, you and all other participants of your group (see below) will participate in a labor market as workers. Each of you will become employed or unemployed at random, independently of each other. Your main task is to vote on an unemployment insurance scheme, which can provide unemployment benefits to (1) all, (2) some or (3) no unemployed members of your group. The most popular choice will be implemented. Your payments will be made based on the implemented insurance scheme and your employment status. The important variables, that you should consider, are: chances of being employed, voting decisions and payments. 
Group composition: At the beginning of every period, all 28 participants will be randomly allocated into groups of seven people. The composition of your group of seven participants will change during the experiment, but its size will remain fixed.

Chances of being employed: In each period, all members of your group, including yourself, will be assigned a chance of being employed, at random and independently of each other. In general, the chances of being employed will, in each period, for all participants be one of the following numbers:

$$
30 \%, \quad 40 \%, \quad 50 \%, \quad 60 \%, \quad 70 \%, \quad 80 \%, \quad 90 \% \text {. }
$$

Remark that your employment chances will change from period to period. However, it will always be the case that two group members have an employment chance of either

$30 \%$ or $40 \%$, and the remaining five group members have an employment chance of at least $50 \%$. In any period, you could either be one of the two group members that have a low employment chance, or one of the five group members that have a high employment chance.

At the beginning of each period, you will be informed about your employment chance and the employment chances of the other members of your group. These chances will be presented in a list and on a graph on your computer screen, in descending order. Your own employment chances will be depicted by blue fonts and bars, while the employment chances of all other group members will be depicted by gray fonts and bars (see also screenshots of the experimental software at the end of this document).

Insurance scheme: After you learn about employment chances but before you know whether you and the other members of your group are employed in the current period, you will be asked to vote on an insurance scheme. You will always have the following three possible choices:

1. Full Insurance. The government provides a universal safety net. If this option is implemented, all unemployed group members receive unemployment benefits and all employed group members pay a tax.

2. Partial Insurance. The government provides a safety net, but only for those group members with at least $50 \%$ chance of being employed. If this option is implemented, all unemployed group members with an employment chance of at least 50\% receive unemployment benefit and all employed group members with an employment chance of at least $50 \%$ pay a tax. On the other hand, those group 
members with employment chances of $30 \%$ and $40 \%$ neither receive benefits nor pay a tax. Remember that, in any period, you could either belong to the five group members that are covered by this insurance scheme, or not. However, you will always know whether you would be covered by the Partial Insurance scheme before you make your choice.

3. No Insurance. If this option is implemented, the government does nothing and there is no insurance policy for unemployed group members.

You will first be asked to state your most preferred choice from the three options above. Next, you will be asked about your second preferred choice: which of the two remaining options do you prefer over the other, in case when your most preferred choice is not implemented (see also screenshots of the experimental software at the end of this document).

You are always allowed to vote. However, this is not the case for all members of your group. After all group members that can vote have made their choice, the most popular policy will be implemented. However, if no option gains majority of the votes, the votes on the second preferred options will be considered. You will then be informed about (1) the implemented policy, (2) whether you were actually employed/unemployed and (3) what is your payoff for that period.

Payments: In the experiment you will collect points where 750 points correspond to $1 €$. The baseline payments in every period are 400 points for employed group members, and 80 points for unemployed group members. This payoff can be modified by taxes for employed, and insurance payments for unemployed group members, depending on the implemented insurance policy.

1. Full Insurance. If this policy choice is implemented, all employed group members pay taxes and all unemployed group members receive unemployment benefits. If you are employed, you will then receive 400 points and pay 100 points for taxes, so that you are left with $\mathbf{3 0 0}$ points. If you are unemployed you receive, on top of the baseline 80 points, an additional 150 points as unemployment benefits from the government, so that you end up with 230 points (see Table). This also holds for all other members of your group.

2. Partial Insurance. If this policy choice is implemented, the insurance policy will only cover all group members with high employment chances. If you belong to the group members with an employment chance of at least 50\%, you will therefore only have to pay a tax of 75 points if you are employed, so that your 


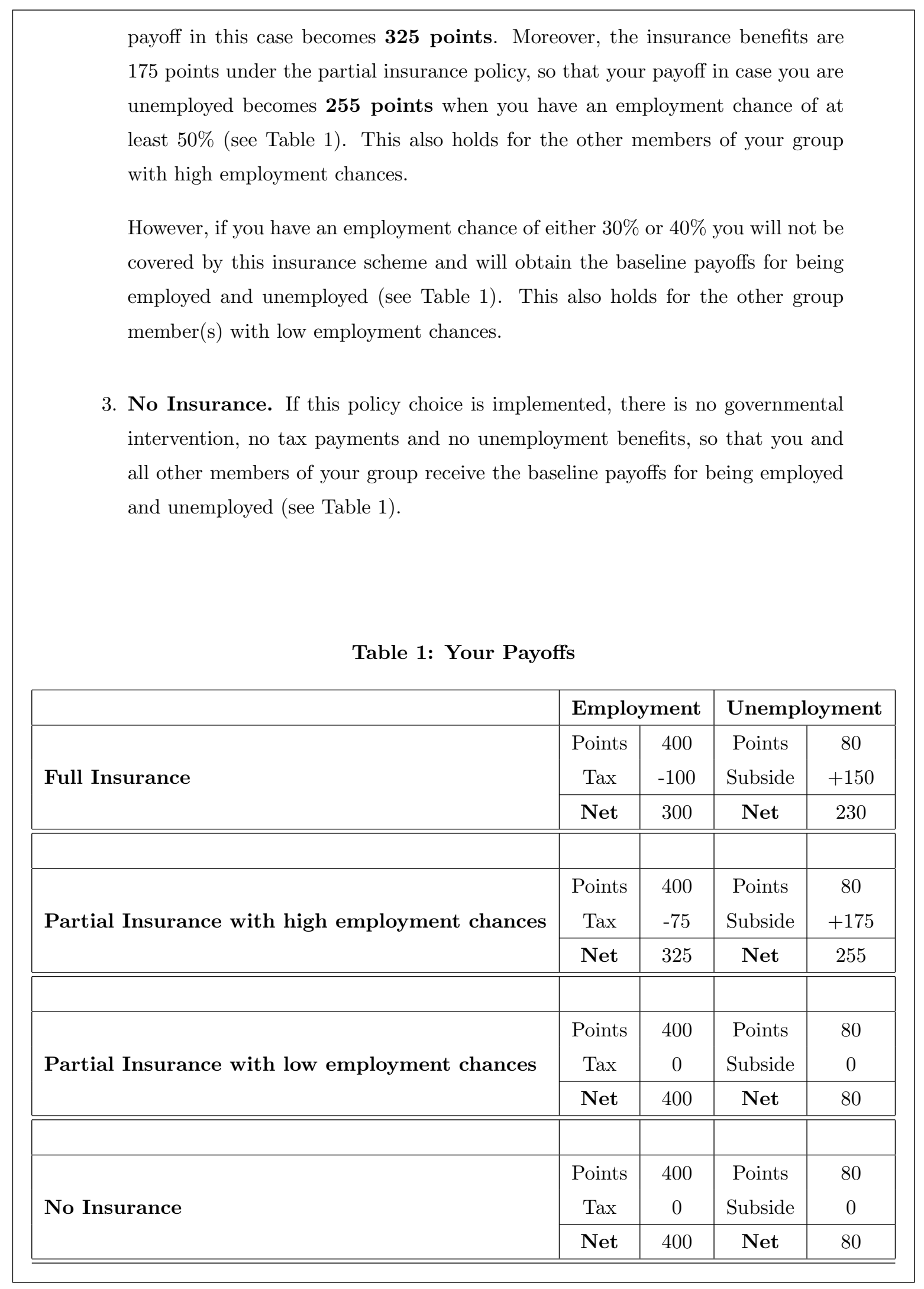




\section{Screenshots of the computer software}

\section{Vorherige Runde}

Das Versicherungssystem, das in der letzten Runde implementiert wurde, war 'Keine Versicherung.', was

bedeutet, dass Sie nicht versichert waren.

Ihre Beschäftigungschancen waren $60 \%$.

Es stellte sich heraus, dass Sie in der vergangenen Runde erwerbstätig waren. Daher beträgt Ihre Auszahlung 400 Punkte.

Die Gesamtzahl der Punkte, die Sie bisher im Experiment gesammelt haben, beträgt jetzt 400 Punkte.

\section{Aktuelle Runde (2/25)}

\section{Beschäftigungschancen}

In dieser Runde beträgt Ihre Beschäftigungschance $\mathbf{8 0 \%}$

Darüber hinaus sind die Beschäftigungschancen für alle Mitglieder Ihrer aktuellen Gruppe (einschließlich Ihnen):

$[80,80,60,50,50,30,30] \%$

\section{Am meisten bevorzugte Wahl}

Welche der folgenden Alternativen ist Ihre am meisten bevorzugte Auswahlmöglichkeit in dieser Runde? (Beachten Sie, dass Sie - da Ihre Beschäftigungschancen ausreichend hoch sind - versichert sind, wenn eine TeilVersicherung umgesetzt wird. )

Voll-Versicherung

Teil-Versicherung

Keine Versicherung

\section{Weiter}

Beschäftigungschancen aller Teilnehmer in Ihrer Gruppe

Die dunkelblaue Spalte zeigt Ihre Beschäftigungschance an

$$
100
$$

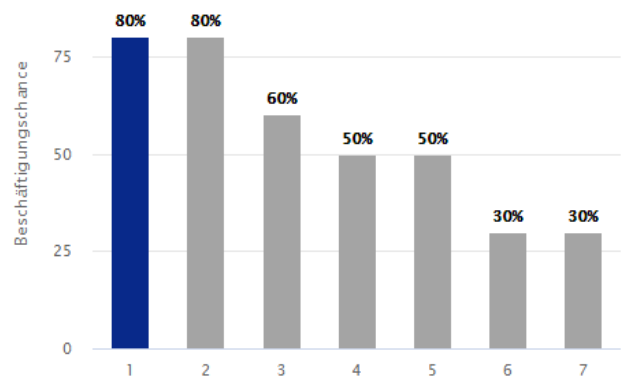

Figure1: Example on computer

\section{Gray Inequality for Green and Purple participants}

The instructions provided to Green and Purple participants in this treatment are similar to the Gray Equality treatment. All participants are unaware of the split into two groups. Nonetheless, the main difference between Green and Purple participants relies on the chances of being employed in every period. While Green participants are always the lucky participants who enjoy chances of being employed of at least 50\%, Purple participants are always the unlucky participants who enjoy chances 
of being employed of either $30 \%$ or $40 \%$.

\section{Colorful Inequality for Green and Purple participants}

This treatment informs about the existence of the two color types. All participants are aware of the split into two groups. The main difference between the instructions provided to Green and Purple participants is based on the chances of being employed in every period. While Green participants are always those who enjoy chances of being employed of at least $50 \%$ because of the color type (as indicated below in the instructions for Green participants), Purple participants are always those who enjoy chances of being employed of either $30 \%$ or $40 \%$ because of the color type.

\section{Experimental instructions and general information}

Welcome to this experiment at the University of Bamberg! The experiment is anonymous, the data from your decisions will only be linked to your station ID, not to your name. You will be paid privately at the end, after all participants have finished the experiment. After the main part of the experiment and before the payment, you will be asked to fill out a short questionnaire.

During the experiment you are not allowed to use your mobile phone or any other communication devices. You are also not allowed to communicate with other participants. If you have a question at any time, please raise your hand and someone will come to your desk.

\section{Experimental economy}

The experiment will last for 25 periods. In each period, you and all other participants of your group (see below) will participate in a labor market as workers. Each of you will become employed or unemployed at random, independently of each other. Your main task is to vote on an unemployment insurance scheme, which can provide unemployment benefits to (1) all, (2) some or (3) no unemployed members of your group. The most popular choice will be implemented. Your payments will be made based on the implemented insurance scheme and your employment status. The important variables, that you should consider, are: chances of being employed, voting decisions and payments. 
Group composition At the beginning of every period, all 28 participants will be randomly allocated into groups of seven people. The composition of your group of seven participants will change during the experiment, but its size will remain fixed. Furthermore, all participants are of one of two different types: green and purple. You belong to the green type. The random allocation to groups is such that, in every period, your group will consist of two participants of the purple type and five participants of the green type (including yourself).

Chances of being employed In each period, all members of your group, including yourself, will be assigned a chance of being employed, at random and independently of each other. In general, the chances of being employed will, in each period, for all participants be one of the following numbers:

$$
30 \%, \quad 40 \%, \quad 50 \%, \quad 60 \%, \quad 70 \%, \quad 80 \%, \quad 90 \% \text {. }
$$

Remark that your employment chances will change from period to period. However, since you are of the green type, your chances of being employed are always at least $50 \%$. The chances of being employed of participants of the purple type, on the other hand, will always be either $30 \%$ or $40 \%$.

At the beginning of each period, you will be informed about your employment chance and the employment chances of the other members of your group. These chances will be presented in a list and on a graph on your computer screen, in descending order. The employment chances of green (purple) group members will be depicted by green (purple) fonts and bars. Your own employment chances will be depicted by dark green fonts and bars (see also screenshots of the experimental software at the end of this document).

Insurance scheme After you learn about employment chances but before you know whether you and the other members of your group are employed in the current period, you will be asked to vote on an insurance scheme. You will always have the following three possible choices:

1. Full Insurance. The government provides a universal safety net. If this option is implemented, all unemployed group members receive unemployment benefits and all employed group members pay a tax.

2. Partial Insurance. The government provides a safety net, but only for green members of your group, including yourself. If this option is implemented, all 
green unemployed group members receive unemployment benefits and all green employed group members pay a tax. On the other hand, purple group members neither receive benefits nor pay a tax.

3. No Insurance. If this option is implemented, the government does nothing and there is no insurance policy for unemployed group members.

You will first be asked to state your most preferred choice from the three options above. Next, you will be asked about your second preferred choice: which of the two remaining options do you prefer over the other, in case when your most preferred choice is not implemented (see also screenshots of the experimental software at the end of this document).

Only green participants can vote; purple participants are not allowed to vote. After all green members of your group vote, the most popular policy will be implemented. However, if no option gains majority of the votes, the votes on the second preferred options will be considered. You will then be informed about (1) the implemented policy, (2) whether you were actually employed/unemployed and (3) what is your payoff for that period.

Payments In the experiment you will collect points where 750 points correspond to $1 €$. The baseline payments in every period are 400 points for employed group members, and 80 points for unemployed group members. This payoff can be modified by taxes for employed, and insurance payments for unemployed group members, depending on the implemented insurance policy.

1. Full Insurance. If this policy choice is implemented, all employed group members pay taxes and all unemployed group members receive unemployment benefits. If you are employed, you will then receive 400 points and pay 100 points for taxes, so that you are left with $\mathbf{3 0 0}$ points. If you are unemployed you receive, on top of the baseline 80 points, an additional 150 points as unemployment benefits from the government, so that you end up with $\mathbf{2 3 0}$ points (see Table 1). This also holds for all other members of your group.

2. Partial Insurance. If this policy choice is implemented, the insurance policy will only cover all green group members (including yourself), who have better chances of being employed than purple group members. Under this insurance policy, you will therefore only have to pay a tax of 75 points if you are employed, so that your payoff in this case becomes $\mathbf{3 2 5}$ points. Moreover, the insurance benefits are 175 points under the partial insurance policy, so that your payoff in 
case you are unemployed becomes 255 points (see Table 1). This also holds for the other green members of your group. Purple group members, however, will not be covered by this insurance scheme and will obtain the baseline payoffs for being employed and unemployed.

3. No Insurance. If this policy choice is implemented, there is no governmental intervention, no tax payments and no unemployment benefits, so that you and all other members of your group receive the baseline payoffs for being employed and unemployed (see Table 1).

Table1: Your Payoffs

\begin{tabular}{|l|c|c|c|c|}
\hline \multirow{3}{*}{ Full Insurance } & \multicolumn{2}{|c|}{ Employment } & \multicolumn{2}{c|}{ Unemployment } \\
\hline & Points & 400 & Points & 80 \\
& Tax & -100 & Subside & +150 \\
\cline { 2 - 5 } & Nett & 300 & Nett & 230 \\
\hline \hline \multirow{4}{*}{ Partial Insurance } & Tax & -75 & Subside & +175 \\
\cline { 2 - 5 } & Nett & 325 & Nett & 255 \\
\hline \hline \multirow{4}{*}{ No Insurance } & & & & 80 \\
\hline & Points & 400 & Points & 80 \\
\cline { 2 - 5 } & Tax & 0 & Subside & 0 \\
\cline { 2 - 5 } & Nett & 400 & Nett & 80 \\
\hline
\end{tabular}




\section{Screenshots of the computer software}

\section{Vorherige Runde}

Das Versicherungssystem, das in der letzten Runde implementiert wurde, war 'Keine Versicherung.', was bedeutet, dass Sie nicht versichert waren.

Ihre Beschäftigungschancen waren $70 \%$.

Es stellte sich heraus, dass Sie in der vergangenen Runde erwerbstätig waren. Daher betrăgt Ihre Auszahlung 400 Punkte.

Die Gesamtzahl der Punkte, die Sie bisher im Experiment gesammelt haben, betrăgt jetzt 400 Punkte.

Aktuelle Runde (2/25)

\section{Beschäftigungschancen}

In dieser Runde beträgt Ihre Beschäftigungschance $60 \%$.

Darüber hinaus sind die Beschăftigungschancen für alle Mitglieder Ihrer aktuellen Gruppe (einschließlich Ihnen):

$[90,70,70,60,60,40,30] \%$.

\section{Am meisten bevorzugte Wahl}

Welche der folgenden Alternativen ist Ihre am meisten bevorzugte Auswahlmöglichkeit in dieser Runde?

( Beachten Sie, dass Sie - da Sie ein grüner Spieler sind -

versichert sind, wenn eine Teil-Versicherung umgesetzt wird. )
Beschäftigungschancen aller Teilnehmer in Ihrer Gruppe

Die dunkelgrüne Spalte zeigt lhre Beschäftigungschance an

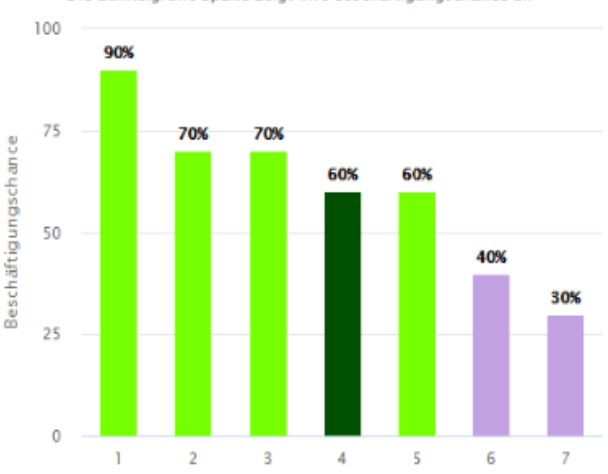

\footnotetext{
Voll-Versicherung

Teil-Versicherung

Keine Versicherung
}

Figure1: Example on computer

\section{Colorful Equality for Green and Purple participants}

This treatment informs about the existence of the two color types. All participants are aware of the split into two groups. Moreover, Green and Purple participants always draw from the same probability employment distribution. The main difference between the instructions provided to Green and Purple participants in this treatment is based on the expected payoffs for Green participants. While Green participants have the same expected payoffs by voting for either Full or Partial insurance 
in every period (as indicated below in the instructions for Green participants), Purple participants have the identical expected payoff as in the other treatments.

\section{Experimental instructions and general information}

Welcome to this experiment at the University of Bamberg! The experiment is anonymous, the data from your decisions will only be linked to your station ID, not to your name. You will be paid privately at the end, after all participants have finished the experiment. After the main part of the experiment and before the payment, you will be asked to fill out a short questionnaire.

During the experiment you are not allowed to use your mobile phone or any other communication devices. You are also not allowed to communicate with other participants. If you have a question at any time, please raise your hand and someone will come to your desk.

\section{Experimental economy}

The experiment will last for 25 periods. In each period, you and all other participants of your group (see below) will participate in a labor market as workers. Each of you will become employed or unemployed at random, independently of each other. Your main task is to vote on an unemployment insurance scheme, which can provide unemployment benefits to (1) all, (2) some or (3) no unemployed members of your group. The most popular choice will be implemented. Your payments will be made based on the implemented insurance scheme and your employment status. The important variables, that you should consider, are: chances of being employed, voting decisions and payments.

Group composition: At the beginning of every period, all 28 participants will be randomly allocated into groups of seven people. The composition of your group of seven participants will change during the experiment, but its size will remain fixed. Furthermore, all participants belong to one of in total two types: green and purple. You belong to the green type. The random assignment to the groups is selected so that your group consists in every period of two participants of the purple type and five participants of the green type (including you). 
Chances of being employed: In each period, all members of your group, including yourself, will be assigned a chance of being employed, at random and independently of each other. In general, the chances of being employed will, in each period, for all participants be one of the following numbers:

$$
30 \%, \quad 40 \%, \quad 50 \%, \quad 60 \%, \quad 70 \%, \quad 80 \%, \quad 90 \% \text {. }
$$

Remark that your employment chances will change from period to period. However, it will always be the case that two group members have an employment chance of either $30 \%$ or $40 \%$, and the remaining five group members have an employment chance of at least $50 \%$. This distribution is independent on whether the group members are green or purple. Hence you can be in every period either one of the two group members that have a low employment chance, or one of the five group members that have a high employment chance.

At the beginning of each period, you will be informed about your employment chance and the employment chances of the other members of your group. These chances will be presented in a list and on a graph on your computer screen, in descending order. The employment chances of the green (purple) group members are shown in green (purple) font and a green (purple) bar. Your own chances of employment will be shown in dark green font and dark green bar (see screenshots of the experimental software at the end of this document).

Insurance scheme: After you learn about employment chances but before you know whether you and the other members of your group are employed in the current period, you will be asked to vote on an insurance scheme. You will always have the following three possible choices:

1. Full Insurance. The government provides a universal safety net. If this option is implemented, all unemployed group members receive unemployment benefits and all employed group members pay a tax.

2. Partial Insurance. The government provides a safety net, but only for the green group members (including yourself). If this option is implemented, all green unemployed group members receive unemployment benefits and all green employed group members pay a tax. Purple group members, however, neither receive benefits nor pay a tax.

3. No Insurance. If this option is implemented, the government does nothing and there will be no insurance scheme for unemployed group members. 
You will first be asked to state your most preferred choice from the three options above. Next, you will be asked about your second preferred choice: which of the two remaining options do you prefer over the other, in case when your most preferred choice is not implemented (see also screenshots of the experimental software at the end of this document).

Only green participants (including you) are allowed to vote; purple participants are not allowed to vote. After all green members of your group made their choice, the the most popular insurance scheme will be implemented. However, if no option gains majority of the votes, the votes on the second preferred options will be considered. You will then be informed about (1) the implemented scheme, (2) whether you were actually employed/unemployed and (3) what is your payoff for that period.

Payments: In the experiment you will collect points where 750 points correspond to $1 €$. In the experiment you will collect points, whereas 750 points equal 1 Euro. The baseline payments in every period are 400 points for employed group members, and 80 points for unemployed group members. This payoff can be modified by taxes for employed, and insurance payments for unemployed group members, depending on the implemented insurance scheme.

1. Full Insurance. If this choice is implemented, all employed group members pay taxes and all unemployed group members receive unemployment benefits. If you are employed, you will then receive 400 points and pay 100 points for taxes, so that you are left with $\mathbf{3 0 0}$ points. If you are unemployed you receive, on top of the baseline 80 points, an additional 150 points as unemployment benefits from the government, so that you end up with $\mathbf{2 3 0}$ points (see Table 1). This also holds for all other members of your group.

2. Partial Insurance. If this choice is implemented, the insurance scheme will only cover all green group members (including yourself).Under this insurance scheme, you will receive the same payoffs as under the full insurance. This means that in case you are employed you have to pay a tax of 100 points, so that your payoff in this case becomes 300 points. Moreover, the insurance benefits are 150 points, so that your payoff in case you are unemployed becomes 230 points (see Table 1). This also holds for the other green members of your group. However, purple group members will not be covered by this insurance scheme and will obtain the baseline payoffs for being employed and unemployed.

3. No Insurance. If this choice is implemented, there is no governmental inter- 
vention, no tax payments and no unemployment benefits, so that you and all other members of your group receive the baseline payoffs for being employed (400 points) and unemployed (80 points). See Table 1.

Table1: Your Payoffs

\begin{tabular}{|l|c|c|c|c|}
\hline \multirow{3}{*}{ Full Insurance } & \multicolumn{2}{|c|}{ Employment } & \multicolumn{2}{c|}{ Unemployment } \\
\hline & Points & 400 & Points & 80 \\
& Tax & -100 & Subside & +150 \\
\cline { 2 - 5 } & Netto & 300 & Netto & 230 \\
\hline \hline \multirow{4}{*}{ Partial Insurance } & Tax & -100 & Subside & +150 \\
\cline { 2 - 5 } & Netto & 300 & Netto & 230 \\
\hline \hline \multirow{4}{*}{ No Insurance } & & & & 80 \\
\hline & Points & 400 & Points & 80 \\
\cline { 2 - 5 } & Netto & 400 & Subside & 0 \\
\cline { 2 - 5 } & Netto & 80 \\
\hline
\end{tabular}




\section{Screenshots of the computer software}

\section{Vorherige Runde}

Das Versicherungssystem, das in der letzten Runde implementiert wurde, war 'Keine Versicherung.', was bedeutet, dass Sie nicht versichert waren.

Ihre Beschäftigungschancen waren $60 \%$.

Es stellte sich heraus, dass Sie in der vergangenen Runde erwerbstätig waren. Daher beträgt Ihre Auszahlung 400 Punkte.

Die Gesamtzahl der Punkte, die Sie bisher im Experiment gesammelt haben, beträgt jetzt 400 Punkte.

\section{Aktuelle Runde (2/25)}

\section{Beschäftigungschancen}

In dieser Runde beträgt Ihre Beschäftigungschance $30 \%$,

Darüber hinaus sind die Beschäftigungschancen für alle Mitglieder Ihrer aktuellen Gruppe (einschließlich Ihnen):

$[90,90,80,70,60,30,30] \%$

\section{Am meisten bevorzugte Wahl}

Welche der folgenden Alternativen ist Ihre am meisten bevorzugte Auswahlmöglichkeit in dieser Runde?

(Beachten Sie, dass Sie - da Sie ein grüner Spieler sind -

versichert sind, wenn eine Teil-Versicherung umgesetzt wird. )
Voll-Versicherung
Teil-Versicherung
Keine Versicherung

Beschäftigungschancen aller Teilnehmer in Ihrer Gruppe

Die dunkelgrüne Spalte zeigt Ihre Beschäftigungschance an

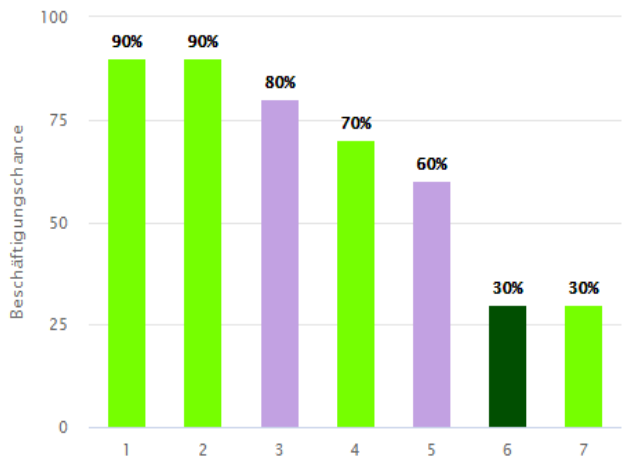

Figure 1: Example on computer 


\section{Survey}

\section{General Information}

For green and purple participants.

Question 1: How old are you?

Question 2: What is your gender?

Question 3: What is the highest degree you have achieved so far

Question 4: What degree are you currently aiming for

Question 5: What is your field of study

Question 6: What is your nationality

Question 7: In which country were you born?

Question 8: Were your parents born in the same country as you?

\section{Risk Aversion}

For green and purple participants.

You can indicate your preferences below each time whether you would hypothetically participate in a lottery game or would like to obtain a safe outcome. In the case that you would like to participate in the lottery game, you have a $50 \%$ probability of winning 150 and a $50 \%$ probability of receiving nothing. For each of the following ten cases below, please indicate whether you would prefer to participate in the lottery game or you would like to receive a safe outcome. Please note that this is only a game and you will not receive any additional payment. 


\begin{tabular}{|c|c|}
\hline Option A (lottery game) & Option B (safe outcome) \\
\hline 150 (50\% probability) or 0 (50\% probability) & 10 \\
150 (50\% probability) or 0 (50\% probability) & 20 \\
150 (50\% probability) or 0 (50\% probability) & 30 \\
150 (50\% probability) or 0 (50\% probability) & 40 \\
150 (50\% probability) or 0 (50\% probability) & 50 \\
150 (50\% probability) or 0 (50\% probability) & 60 \\
150 (50\% probability) or 0 (50\% probability) & 70 \\
150 (50\% probability) or 0 (50\% probability) & 80 \\
150 (50\% probability) or 0 (50\% probability) & 90 \\
150 (50\% probability) or 0 (50\% probability) & 100 \\
\hline
\end{tabular}

Table 3.15: Risk Aversion

\section{Decisions}

\section{For green participants:}

Please indicate, on a scale from 1 to 10, to what extent you agree with the following statements. While the number 1 indicates that you strongly disagree with the statement, the number 10 indicates that you strongly agree with the statement.

Control 1: The decisions I made depended on my own employment opportunities

Control 2: The decisions I made depended on the employment opportunities of the other members of my group

Control 3: The decisions I made were based on my employment opportunities in previous periods

Control 4: The decisions I made depended on the insurance systems implemented in previous periods

Control 5: My decisions were based on the knowledge that I was a green person? (only asked in the Colorful treatments, i.e. CE \& CI) 
For purple participants:

Please indicate, on a scale from 1 to 10, to what extent you agree with the following statements. While the number 1 indicates that you strongly disagree with the statement, the number 10 indicates that you strongly agree with the statement.

Control 1: My stated preferences depended on my own employment opportunities

Control 2: My stated preferences depended on the employment opportunities of the other members of my group

Control 3: My stated preferences were based on my employment opportunities in previous periods

Control 4: My stated preferences depended on the insurance systems implemented in previous periods

Control 5: My decisions were based on the knowledge that I was a purple person? (only asked in the Colorful treatments, i.e. CE \& CI)

\section{Demographic controls}

For green and purple participants.

Please indicate, on a scale from 1 to 10 , to what extent you agree with the following statements. While the number 1 indicates that you strongly disagree with the statement, the number 10 indicates that you strongly agree with the statement.

Control 1: Germany spends too much money on immigrants

Control 2: Germany spends too little money on immigrants

Control 3: Germany has currently accepted too many immigrants

Control 4: Germany should accept more immigrants

Control 5: Immigrants have integrated well into German society 
Control 6: Germany has a problem with immigrants who do not integrate into society

\section{Preferences CE as a base outcome}

Table 3.16: First and second decisions with linear employment probabilities. No_Partial insurance and CE treatment as base outcome

\begin{tabular}{lccccc}
\hline \hline Variables & 1.Full_No & 2.Full_Partial & 3.No_Full & 5.Partial_Full & 6.Partial_No \\
\hline probability & $-17.23^{* * *}$ & $-18.95^{* * *}$ & $-4.630^{* * *}$ & $-13.90^{* * *}$ & $-6.621^{* * *}$ \\
CI & 2.184 & $3.184^{*}$ & -0.816 & $3.403^{* * *}$ & $2.286^{* * *}$ \\
GE & 0.316 & -1.397 & $-2.175^{*}$ & -0.352 & 0.107 \\
GI & -2.099 & -0.836 & $-3.922^{* * *}$ & 1.055 & $1.295^{* *}$ \\
ind. RE & 1 & $0.872^{* * *}$ & $0.515^{* * *}$ & $0.409^{* * *}$ & $0.206^{* * *}$ \\
Constant & $9.279^{* * *}$ & $12.76^{* * *}$ & $3.655^{* * *}$ & $9.798^{* * *}$ & $4.091^{* * *}$ \\
\hline Observations & 1900 & & & \\
\hline \hline
\end{tabular}

Table 3.17: First and second decisions with non-linear employment probabilities. No_Partial insurance, CE treatment and Ch5060 as base outcome

\begin{tabular}{lccccc}
\hline \hline Variables & 1.Full_No & 2.Full_Partial & 3.No_Full & 5.Partial_Full & 6.Partial_No \\
\hline Ch3040 & $2.933^{* * *}$ & $2.249^{* * *}$ & -0.459 & 0.478 & $-1.900^{* *}$ \\
Ch7080 & $-2.887^{* * *}$ & $-3.734^{* * *}$ & $-0.672^{*}$ & $-3.208^{* * *}$ & $-1.423^{* * *}$ \\
Ch90 & $-4.255^{* * *}$ & $-5.604^{* * *}$ & $-1.613^{* * *}$ & $-4.965^{* * *}$ & $-2.824^{* * *}$ \\
CI & 1.822 & 2.833 & -1.042 & $2.995^{* * *}$ & $2.072^{* * *}$ \\
GE & 0.222 & -1.341 & $-2.259^{* *}$ & -0.435 & -0.0321 \\
GI & -1.820 & -0.708 & $-4.056^{* * *}$ & 0.893 & $1.121^{* *}$ \\
ind. RE & 1 & $0.853^{* * *}$ & $0.528^{* * *}$ & $0.385^{* * *}$ & $0.206^{* * *}$ \\
Constant & -0.927 & $2.221^{*}$ & 1.018 & $2.517^{* * *}$ & $0.764^{*}$ \\
\hline Observations & 1900 & & & \\
\hline \hline
\end{tabular}




\section{Preferences GI as the benchmark}

Table 3.18: First decision with continuous employment chances. No insurance and GI treatment as base outcome

\begin{tabular}{|c|c|c|c|c|}
\hline Variables & Full (1) & Full (2) & Partial(1) & Partial(2) \\
\hline probability & $-14.84^{* * *}$ & $-14.96^{* * *}$ & $-11.44^{* * *}$ & $-11.50^{* * *}$ \\
\hline $\mathrm{CE}$ & 0.0966 & -0.110 & $-2.172^{* * *}$ & $-2.115^{* * *}$ \\
\hline $\mathrm{CI}$ & $2.274^{*}$ & 2.239 & $0.964^{*}$ & 0.941 \\
\hline GE & -1.053 & -1.140 & $-1.266^{* *}$ & $-1.126^{* *}$ \\
\hline poorGE & -0.251 & -0.297 & $-3.650^{* * *}$ & $-3.666^{* * *}$ \\
\hline M1[sub_id] & 1 & 1 & $0.373^{* * *}$ & $0.367^{* * *}$ \\
\hline employed_Freq & & -0.320 & & -0.734 \\
\hline insured_Freq & & 0.216 & & -0.138 \\
\hline full_Freq & & 0.239 & & 0.644 \\
\hline partial_Freq & & -0.0231 & & 0.476 \\
\hline employed_insured_Freq & & -0.553 & & 0.548 \\
\hline Constant & $9.463^{* * *}$ & $9.875^{* * *}$ & $9.770^{* * *}$ & $9.659^{* * *}$ \\
\hline Observations & 1900 & & & 1900 \\
\hline
\end{tabular}


Table 3.19: Employment chance + treatments

\begin{tabular}{|c|c|c|c|c|}
\hline Variables & Full (1) & Full (2) & $\operatorname{Partial}(1)$ & Partial $(2)$ \\
\hline Ch3040 & $2.360^{* * *}$ & $2.656^{* * *}$ & $1.884^{* * *}$ & $1.825^{* * *}$ \\
\hline Ch7080 & $-3.260 * * *$ & $-2.614^{* * *}$ & $-2.388^{* * *}$ & $-2.024^{* * *}$ \\
\hline Ch90 & $-4.855^{* * *}$ & $-3.585 * * *$ & $-3.888^{* * *}$ & $-3.320^{* * *}$ \\
\hline $\mathrm{CE}$ & 0.188 & -0.0944 & $-2.091^{* * *}$ & $-2.987 * * *$ \\
\hline $\mathrm{CI}$ & $2.262^{*}$ & $1.338^{*}$ & $0.978^{*}$ & $-1.129^{* * *}$ \\
\hline GE & -1.040 & -0.196 & $-1.233^{* *}$ & $-1.110^{* * *}$ \\
\hline poorGE & 0.0564 & 0.0184 & $-3.438^{* * *}$ & $-3.343^{* * *}$ \\
\hline M1[sub_id $]$ & 1 & 1 & $0.373^{* * *}$ & $-0.350^{* *}$ \\
\hline employed_Freq & & $-1.684^{* *}$ & & -1.018 \\
\hline insured_Freq & & 0.713 & & -0.766 \\
\hline full_Freq & & 1.227 & & $3.007^{* * *}$ \\
\hline partial_Freq & & -0.423 & & 0.374 \\
\hline employed_insured_Freq & & 0.881 & & 0.836 \\
\hline Constant & 1.369 & 0.450 & $3.446^{* * *}$ & $2.923^{* * *}$ \\
\hline Observations & 1900 & & & 1900 \\
\hline
\end{tabular}

Table 3.20: First and second decisions with linear employment probabilities. No_Partial insurance and GI treatment as base outcome

\begin{tabular}{|c|c|c|c|c|c|}
\hline Variables & 1.Full_No & 2.Full_Partial & 3.No_Full & 5.Partial_Full & 6.Partial_No \\
\hline probability & $-12.48^{* * *}$ & $-19.45^{* * *}$ & $-4.174^{* * *}$ & $-16.64^{* * *}$ & $-7.872^{* * *}$ \\
\hline $\mathrm{CE}$ & 1.270 & 0.358 & $3.836^{* * *}$ & -1.190 & $-1.511^{* * *}$ \\
\hline $\mathrm{CI}$ & $3.757^{* *}$ & $2.845^{*}$ & $2.727^{* *}$ & $2.151^{* *}$ & 0.661 \\
\hline GE & -0.481 & -1.536 & 1.169 & -1.093 & $-1.157^{* *}$ \\
\hline employed_Freq & 0.277 & -1.407 & 0.518 & -1.022 & -0.297 \\
\hline insured_Freq & -1.558 & 0.0796 & -0.387 & -0.253 & -0.514 \\
\hline full_Freq & 1.803 & 0.362 & -0.329 & -0.206 & 1.629 \\
\hline partial_Freq & 0.781 & 0.130 & 0.204 & 0.249 & 1.198 \\
\hline employed_insured_Freq & -0.934 & 0.0817 & -1.128 & 0.773 & -0.112 \\
\hline poorGE & 0.958 & -0.956 & 0.650 & $-4.171^{* * *}$ & $-3.722^{* * *}$ \\
\hline M1[sub_id] & 1 & $1.222^{* * *}$ & $0.730^{* * *}$ & $0.682^{* * *}$ & $0.311^{* * *}$ \\
\hline Constant & $6.942^{* * *}$ & $13.42^{* * *}$ & 0.0799 & $13.18^{* * *}$ & $6.090^{* * *}$ \\
\hline Observations & 1900 & & & & \\
\hline
\end{tabular}


Table 3.21: First and second decisions with non-linear employment probabilities. No_Partial insurance, GI treatment and Ch5060 as base outcome

\begin{tabular}{lccccc}
\hline \hline Variables & 1.Full_No & 2.Full_Partial & 3.No_Full & 5.Partial_Full & 6.Partial_No \\
\hline Ch3040 & $2.933^{* * *}$ & $2.249^{* * *}$ & -0.459 & 0.478 & $-1.900^{* *}$ \\
Ch7080 & $-2.887^{* * *}$ & $-3.734^{* * *}$ & $-0.672^{*}$ & $-3.208^{* * *}$ & $-1.423^{* * *}$ \\
Ch90 & $-4.255^{* * *}$ & $-5.604^{* * *}$ & $-1.613^{* * *}$ & $-4.965^{* * *}$ & $-2.824^{* * *}$ \\
CE & 1.820 & 0.708 & $4.056^{* * *}$ & -0.893 & $-1.121^{* *}$ \\
CI & $3.642^{*}$ & $3.541^{*}$ & $3.014^{* *}$ & $2.102^{* *}$ & $0.951^{*}$ \\
GE & 2.042 & -0.633 & 1.796 & -1.328 & $-1.153^{* *}$ \\
M1[sub_id] & 1 & $0.853^{* * *}$ & $0.528^{* * *}$ & $0.385^{* * *}$ & $0.206^{* * *}$ \\
Constant & $-2.747^{*}$ & 1.514 & $-3.038^{* * *}$ & $3.410^{* * *}$ & $1.885^{* * *}$ \\
\hline Observations & 1900 & & & & \\
\hline \hline & $* p<0.10, * * p<0.05, * * * p<0.01$ & & & \\
\hline
\end{tabular}

Table 3.22: First decision without employment chances. No insurance and CE treatment as base outcome

\begin{tabular}{lcc}
\hline \hline Variables & Full & Partial \\
\hline CI & 0.0753 & $0.657^{* * *}$ \\
GE & $-1.637^{* *}$ & $0.551^{* *}$ \\
GI & $-1.304^{*}$ & $1.297^{* * *}$ \\
poorGE & $4.443^{* * *}$ & 0.123 \\
ind.RE & 1 & $-0.291^{* *}$ \\
Constant & -0.243 & $-0.424^{* *}$ \\
\hline Observations & 1900 & \\
\hline \hline & $* p<0.10,{ }^{* *} p<0.05,{ }^{* * *} p<0.01$
\end{tabular}


Table 3.23: Frequency probability30_40

\begin{tabular}{|l|cccc|}
\hline pairwise decision and & \multicolumn{4}{|c|}{ treatment } \\
probability 30_40 & CE & CI & GE & GI \\
\hline probability30_40=0 & & & & \\
Full_No & 20 & 34 & 8 & 4 \\
Full_Partial & 86 & 161 & 25 & 59 \\
No_Full & 69 & 4 & 15 & 2 \\
No_Partial & 108 & 99 & 140 & 91 \\
Partial_Full & 48 & 136 & 120 & 154 \\
Partial_No & 34 & 66 & 61 & 90 \\
\hline probability30_40=1 & & & & \\
Full_No & 3 & & 34 & \\
Full_Partial & 64 & & 75 & \\
No_Full & & & 3 & \\
No_Partial & 5 & & 6 & \\
Partial_Full & 62 & & 12 & \\
Partial_No & 1 & & 1 \\
\hline
\end{tabular}




\section{Auxiliary estimations}

Table 3.24: First decision with continuous employment chances. No insurance and CE treatment as base outcome

\begin{tabular}{lcccc}
\hline \hline Variables & Full $(1)$ & Full $(2)$ & Partial(1) & Partial(2) \\
\hline probability & $-14.84^{* * *}$ & $-14.96^{* * *}$ & $-11.44^{* * *}$ & $-11.50^{* * *}$ \\
CI & 2.177 & $2.349^{*}$ & $3.136^{* * *}$ & $3.056^{* * *}$ \\
GE & -1.149 & -1.030 & $0.906^{*}$ & $0.990^{*}$ \\
GI & -0.0966 & 0.110 & $2.172^{* * *}$ & $2.115^{* * *}$ \\
poorGE & -0.251 & -0.297 & $-3.650^{* * *}$ & $-3.666^{* * *}$ \\
employed_Freq & & -0.320 & & -0.734 \\
insured_Freq & & 0.216 & & -0.138 \\
full_Freq & & 0.239 & & 0.644 \\
partial_Freq & & -0.0231 & & 0.476 \\
employed_insured_Freq & & -0.553 & & 0.548 \\
ind. RE & 1 & 1 & $0.308^{* * *}$ & $0.367^{* * *}$ \\
Constant & $9.560^{* * *}$ & $9.764^{* * *}$ & $7.597^{* * *}$ & $7.544^{* * *}$ \\
\hline Observations & & 1900 & & 1900 \\
\hline \hline
\end{tabular}

$* p<0.10,{ }^{* *} p<0.05,{ }^{* * *} p<0.01$ 
Table 3.25: First decision with non-linear employment probabilities. CE treatment and Ch5060 as base outcome

\begin{tabular}{lcccc}
\hline \hline Variables & Full (1) & Full $(2)$ & Partial (1) & Partial $(2)$ \\
\hline Ch3040 & $2.360^{* * *}$ & $2.656^{* * *}$ & $1.884^{* * *}$ & $1.825^{* * *}$ \\
Ch7080 & $-3.260^{* * *}$ & $-2.614^{* * *}$ & $-2.388^{* * *}$ & $-2.024^{* * *}$ \\
Ch90 & $-4.855^{* * *}$ & $-3.585^{* * *}$ & $-3.888^{* * *}$ & $-3.320^{* * *}$ \\
CI & 2.074 & $1.433^{*}$ & $3.068^{* * * *}$ & $1.858^{* * *}$ \\
GE & -1.227 & -0.101 & 0.858 & $1.877^{* * *}$ \\
GI & -0.188 & 0.0945 & $2.091^{* * *}$ & $2.987^{* * *}$ \\
poorGE & 0.0564 & 0.0184 & $-3.438^{* * *}$ & $-3.343^{* * *}$ \\
employed_Freq & & $-1.684^{* *}$ & & -1.018 \\
insured_Freq & & 0.714 & & -0.766 \\
full_Freq & & 1.227 & & $3.007^{* * *}$ \\
partial_Freq & & -0.423 & & 0.374 \\
employed_insured_Freq & & 0.881 & & 0.836 \\
ind. RE & 1 & $0.314^{* * *}$ & & $-0.350^{* *}$ \\
Constant & $1.556^{*}$ & 0.356 & $1.356^{* * *}$ & -0.0642 \\
\hline Observations & 1900 & & & 1900 \\
\hline \hline
\end{tabular}

${ }^{*} p<0.10,{ }^{* *} p<0.05,{ }^{* * *} p<0.01$ 
Table 3.26: First and second decisions with continuous employment probabilities. No_Partial insurance and $\mathrm{CE}$ treatment as base outcome

\begin{tabular}{lccccc}
\hline \hline Variables & 1.Full_No & 2.Full_Partial & 3.No_Full & 5.Partial_Full & 6.Partial_No \\
\hline probability & $-12.48^{* * *}$ & $-19.45^{* * *}$ & $-4.174^{* * *}$ & $-16.64^{* * *}$ & $-7.872^{* * *}$ \\
CI & $2.487^{*}$ & 2.487 & -1.109 & $3.341^{* * *}$ & $2.172^{* * *}$ \\
GE & -1.751 & -1.894 & $-2.667^{* *}$ & 0.0963 & 0.354 \\
GI & -1.270 & -0.358 & $-3.836^{* * *}$ & 1.190 & $1.511^{* * *}$ \\
poorGE & 0.958 & -0.956 & 0.650 & $-4.171^{* * *}$ & $-3.722^{* * *}$ \\
employed_Freq & 0.277 & -1.407 & 0.518 & -1.022 & -0.297 \\
insured_Freq & -1.558 & 0.0796 & -0.387 & -0.253 & -0.514 \\
full_Freq & 1.803 & 0.362 & -0.329 & -0.206 & 1.629 \\
partial_Freq & 0.781 & 0.130 & 0.204 & 0.249 & 1.198 \\
employed_insured_Freq & -0.934 & 0.0817 & -1.128 & 0.773 & -0.112 \\
ind. RE & 1 & $1.222^{* * *}$ & $0.730^{* * *}$ & $0.682^{* * *}$ & $0.311^{* * *}$ \\
Constant & $8.212^{* * *}$ & $13.78^{* * *}$ & $3.916^{* * *}$ & $11.99^{* * *}$ & $4.579^{* * *}$ \\
\hline Observations & 1900 & & & & \\
\hline \hline
\end{tabular}

${ }^{*} p<0.10,{ }^{* *} p<0.05,{ }^{* * *} p<0.01$ 


\section{Bibliography}

Abou-Chadi, T. (2016), 'Niche Party Success and Mainstream Party Policy Shifts - How Green and Radical Right Parties Differ in Their Impact', British Journal of Political Science 46(2), 417-436.

Abrigo, M. R. and Love, I. (2016), 'Estimation of Panel Vector Autoregression in Stata', The Stata Journal 16(3), 778-804.

Acemoglu, D., Aghion, P. and Zilibotti, F. (2006), 'Distance to Frontier, Selection, and Economic Growth', Journal of the European Economic Association 4(1), 37-74.

Acemoglu, D., Naidu, S., Restrepo, P. and Robinson, J. A. (2015), Democracy, Redistribution, and Inequality, in 'Handbook of Income Distribution', Vol. 2, 1885-1966, Elsevier.

Aghion, P. and Bolton, P. (1997), 'A Theory of Trickle-Down Growth and Development', The Review of Economic Studies 64(2), 151-172.

Aghion, P., Caroli, E. and Garcia-Penalosa, C. (1999), 'Inequality and Economic Growth: The Perspective of the New Growth Theories', Journal of Economic Literature 37(4), 1615-1660.

Akerlof, G. A. and Kranton, R. E. (2000), 'Economics and Identity', The Quarterly Journal of Economics 115(3), 715-753.

Alesina, A. and Ferrara, E. L. (2005), 'Ethnic Diversity and Economic Performance', Journal of Economic Literature 43(3), 762-800.

Alesina, A. and Glaeser, E. (2004), Fighting Poverty in the US and Europe: A World of Difference, Oxford University Press.

Alesina, A., Glaeser, E. and Sacerdote, B. (2001), 'Why Doesn't the United States Have a EuropeanStyle Welfare System?', Brookings Papers on Economic Activity 2, 187-254.

Alesina, A., Miano, A. and Stantcheva, S. (2019), Immigration and Redistribution. NBER Working Paper, No. 24733, National Bureau of Economic Research.

Alesina, A. and Rodrik, D. (1994), 'Distributive Politics and Economic Growth', The Quarterly Journal of Economics 109(2), 465-490. 
Alvaredo, F., Chancel, L., Piketty, T., Saez, E. and Zucman, G. (2018), World Inequality Report 2018, Belknap Press.

Anderson, C. J. (1995), Blaming the Government: Citizens and the Economy in Five European Democracies, New York: M. E. Sharpe.

Anderson, C. J. and Beramendi, P. (2012), 'Left Parties, Poor Voters, and Electoral Participation in Advanced Industrial Societies', Comparative Political Studies 45(6), 714-746.

Anelli, M., Colantone, I. and Stanig, P. (2019), We Were the Robots: Automation and Voting Behavior in Western Europe. IZA Discussion Papers, No. 12485, Institute of Labor Economics (IZA), Bonn.

Arellano, M. and Bond, S. (1991), 'Some Tests of Specification for Panel Data: Monte Carlo Evidence and an Application to Employment Equations', The Review of Economic Studies 58(2), 277-297.

Arellano, M. and Bover, O. (1995), 'Another look at the instrumental variable estimation of errorcomponents models', Journal of Econometrics 68(1), 29-51.

Arrow, K. J. and Debreu, G. (1954), 'Existence of an Equilibrium for a Competitive Economy', Econometrica 22, 265-290.

Arzheimer, K. (2013), Working-class parties 2.0? Competition between centre left and extreme right parties. In: J. Rydgren (eds.), Class Politics and the Radical Right, 75-90.

Arzheimer, K. and Carter, E. (2006), 'Political opportunity structures and right-wing extremist party success', European Journal of Political Research 45(3), 419-443.

Atems, B. and Jones, J. (2015), 'Income inequality and economic growth: a panel VAR approach', Empirical Economics 48(4), 1541-1561.

Atkinson, A. B. (1970), 'On the measurement of inequality', Journal of Economic Theory 2, 244-263.

Atkinson, A. B., Piketty, T. and Saez, E. (2011), 'Top incomes in the long run of history', Journal of Economic Literature 49(1), 3-71.

Awasthi, V. and Pratt, J. (1990), 'The Effects of Monetary Incentives on Effort and Decision Performance: The Role of Cognitive Characteristics', The Accounting Review 65, 797-811.

Banerjee, A. V. and Newman, A. F. (1993), 'Occupational Choice and the Process of Development', Journal of Political Economy 101(2), 274-298.

Bank for International Settlements (2017), 'Total Credit to Households and Non-Profit Institutions Serving Households, Adjusted for Breaks, retrieved from FRED, Federal Reserve Bank of St. Louis'.

Barro, R. J. (2000), 'Inequality and Growth in a Panel of Countries', Journal of Economic Growth $\mathbf{5}(1), 5-32$. 
Bartels, L. M. (2014), Ideology and Retrospection in Electoral Responses to the Great Recession. In: N. Bermeo and L. M. Bartels (eds.), Mass Politics in Tough Times: Opinions, Votes and Protest in the Great Recession, Oxford: Oxford University Press, 185-223.

Bartolini, S. (2000), 'The Political Mobilization of the European Left, 1860-1980'.

Beck, T. and Levine, R. (2004), 'Stock markets, banks, and growth: Panel Evidence', Journal of Banking \& Finance 28(3), 423-442.

Benabou, R. (1996), Inequality and Growth. NBER Working Paper 5658, National Bureau of Economic Research.

Benoit, K. and Laver, M. (2006), Party Policy in Modern Democracies, London: Routledge.

Berg, A., Ostry, J. D., Tsangarides, C. G. and Yakhshilikov, Y. (2018), 'Redistribution, inequality, and growth: new evidence', Journal of Economic Growth 23(3), 259-305.

Bergmann, H., Bäck, H. and Saalfeld, T. (2020), Party-System Polarization, Legislative Institutions and Cabinet Survival in 28 Parliamentary Democracies, 1945-2019, Bamberg and Lund.

Betz, H.-G. (1994), Radical right-wing populism in Western Europe, New York: St. Martin's Press.

Bloise, F., Chironi, D. and Pianta, M. (2019), Inequality and elections in Italian regions. MPRA Paper No. 96416, Munich Personal RePEc Archive.

Blundell, R. and Bond, S. (1998), 'Initial conditions and moment restrictions in dynamic panel data models', Journal of Econometrics 87(1), 115-143.

Bolt, J., Inklaar, R., de Jong, H. and van Zanden, J. L. (2018), Rebasing 'Maddison': new income comparisons and the shape of long-run economic development. GGDC Research Memorandum No GD-174, Groningen Growth and Development Centre, University of Groningen.

Bonner, S. E., Hastie, R., Sprinkle, G. B. and Young, S. M. (2000), 'A Review of the Effects of Financial Incentives on Performance in Laboratory Tasks: Implications for Management Accounting', Journal of Management Accounting Research 12(1), 19-64.

Bonner, S. E. and Sprinkle, G. B. (2002), 'The effects of monetary incentives on effort and task performance: theories, evidence, and a framework for research', Accounting, Organizations and Society 27(4-5), 303-345.

Bornschier, S. and Kriesi, H. (2013), The Populist Right, the Working Class, and the Changing Face of Class Politics. In: J. Rydgren (eds.), Class Politics and the Radical Right, London and New York, Routledge, 10-29. 
Börsch-Supan, A., Krieger, U. and Schröder, M. (2013), Respondent incentives, interviewer training and survey participation. SHARE Working Paper Series 12-2013, Survey of Health, Ageing and Retirement in Europe, Max Planck Institute for Social Law and Social Policy, Munich.

Bring, J. (1994), 'How to standardize regression coefficients', The American Statistician 48(3), 209213.

Bry, G. and Boschan, C. (1971), Cyclical Analysis of Time Series: Selected Procedures and Computer Programs. National Bureau of Economic Research.

Buch-Hansen, H. and Koch, M. (2019), 'Degrowth through income and wealth caps?', Ecological Economics 160, 264-271.

Burgoon, B., van Noort, S., Rooduijn, M. and Underhill, G. (2019), 'Positional deprivation and support for radical right and radical left parties', Economic Policy 34(97), 49-93.

Camerer, C. F. and Hogarth, R. M. (1999), 'The Effects of Financial Incentives in Experiments: A Review and Capital-Labor-Production Framework', Journal of Risk and Uncertainty 19(1-3), 7-42.

Campbell, D. T. (1965), Ethnocentric and other altruistic motives, New York: University of Nebraska Press.

Carnes, N. (2013), White-Collar Government: The Hidden Role of Class in Economic Policy Making, University of Chicago Press.

Carnes, N. and Lupu, N. (2015), 'Rethinking the Comparative Perspective on Class and Representation: Evidence from Latin America', American Journal of Political Science 59(1), 1-18.

Caselli, F. and Morelli, M. (2004), 'Bad Politicians', Journal of Public Economics 88(3-4), 759-782.

Chen, D. L., Schonger, M. and Wickens, C. (2016), 'oTree - An open-source platform for laboratory, online, and field experiments', Journal of Behavioral and Experimental Finance 9, 88-97.

Chen, Y. and Li, S. X. (2009), 'Group Identity and Social Preferences', American Economic Review 99(1), 431-57.

Chevalier, A., Elsner, B., Lichter, A. and Pestel, N. (2018), Immigrant Voters, Taxation and the Size of the Welfare State. IZA Discussion Papers, No. 11725, Institute of Labor Economics (IZA), Bonn.

Chinn, M. D. and Ito, H. (2008), 'A New Measure of Financial Openness', Journal of Comparative Policy Analysis 10(3), 309-322.

Choi, I. (2001), 'Unit root tests for panel data', Journal of International Money and Finance 20(2), 249-272.

Cochrane, J. H. (2009), Asset pricing: Revised Edition, Princeton University Press. 
Colantone, I. and Stanig, P. (2018), The Economic Determinants of the 'Cultural Backlash': Globalization and Attitudes in Western Europe. BAFFI CAREFIN Centre Research Paper No. 2018-91.

Costa-Font, J. and Cowell, F. (2015), 'Social identity and redistributive preferences: A survey', Journal of Economic Surveys 29(2), 357-374.

da Silva, M. E. A. (2020), 'Does inequality benefit growth? New evidence using a panel VAR approach', International Journal of Development Issues $\mathbf{1 9}(1)$.

Dabla-Norris, M. E., Kochhar, M. K., Suphaphiphat, M. N., Ricka, M. F. and Tsounta, M. E. (2015), Causes and Consequences of Income Inequality: A Global Perspective. IMF Staff Discussion Note, International Monetary Fund SDN/15/13.

Dahlberg, M., Edmark, K. and Lundqvist, H. (2012), 'Ethnic Diversity and Preferences for Redistribution', Journal of Political Economy 120(1), 41-76.

Dassonneville, R. and Lewis-Beck, M. S. (2014), 'Macroeconomics, economic crisis and electoral outcomes: A national European pool', Acta Politica 49(4), 372-394.

de Bromhead, A., Eichengreen, B. and O'Rourke, K. H. (2012), Right-Wing Political Extremism in the Great Depression, Discussion Papers in Economic and Social History, Number 95, University of Oxford.

De Dominicis, L., De Groot, H. L. and Florax, R. J. (2006), Growth and inequality: A meta-analysis. Tinbergen Institute Discussion Paper, No. 06-064/3, Tinbergen Institute, Amsterdam and Rotterdam.

De La Croix, D. and Doepke, M. (2003), 'Inequality and Gowth: Why Differential Fertility Matters', American Economic Review 93(4), 1091-1113.

Dimick, M., Rueda, D. and Stegmueller, D. (2018), 'Models of Other-Regarding Preferences, Inequality, and Redistribution', Annual Review of Political Science 21, 441-460.

Döring, H. and Manow, P. (2015), 'Parliaments and governments composition database (parlgov): Information on parties, elections and cabinets in modern democracies. Stable version $2015-12$ March 2016'.

Dornbusch, R. and Edwards, S. (1989), Macroeconomic populism in Latin America, No. 2986. National Bureau of Economic Research.

Dreher, A. (2006), 'Does globalization affect growth? Evidence from a new index of globalization', Applied Economics 38(10), 1091-1110.

Duca, J. V. and Saving, J. L. (2016), 'Income Inequality and Political Polarization: Time Series Evidence Over Nine Decades', Review of Income and Wealth 62(3), 445-466. 
Duch, R. M. and Stevenson, R. (2006), 'Assessing the magnitude of the economic vote over time and across nations', Electoral Studies 25(3), 528-547.

Dülmer, H. and Klein, M. (2005), 'Extreme Right-Wing Voting in Germany in a Multilevel Perspective: A Rejoinder to Lubbers and Scheepers', European Journal of Political Research 44(2), 243-263.

Dustmann, C., Vasiljeva, K. and Piil Damm, A. (2019), 'Refugee Migration and Electoral Outcomes', The Review of Economic Studies 86(5), 2035-2091.

Eckel, C. C. and Grossman, P. J. (2005), 'Managing diversity by creating team identity', Journal of Economic Behavior 85 Organization 58(3), 371-392.

Elsässer, L., Hense, S. and Schäfer, A. (2017), ‘,Dem Deutschen Volke “? Die ungleiche Responsivität des Bundestags', Zeitschrift für Politikwissenschaft 27(2), 161-180.

Engler, S. and Weisstanner, D. (2020), 'The threat of social decline: income inequality and radical right support', Journal of European Public Policy 28(2), 153-173.

Erikson, R. S., MacKuen, M. B. and Stimson, J. A. (2002), The Macro Polity, Cambridge University Press.

Falter, J. W. (2013), 'Voter Movements to the NSDAP 1924-1933. Methodical Problems - Empirircally Founded Insights - Unresolved Questions', Historical Social Research 1(1), 49-89.

Ferraz, C. and Finan, F. (2009), Motivating Politicians: The Impacts of Monetary Incentives on Quality and Performance. NBER Working Paper, No. 14906, National Bureau of Economic Research.

Fetzer, T. (2019), 'Did Austerity Cause Brexit?', American Economic Review 109(11), 3849-86.

Fisher, S. D. and Renwick, A. (2018), 'The UK's referendum on EU membership of June 2016: how expectations of Brexit's impact affected the outcome', Acta Politica 53(4), 590-611.

Forbes, K. J. (2000), 'A Reassessment of the Relationship Between Inequality and Growth', American Economic Review 90(4), 869-887.

Fraile, M. and Lewis-Beck, M. S. (2014), 'Economic vote instability: Endogeneity or restricted variance? Spanish panel evidence from 2008 and 2011', European Journal of Political Research $\mathbf{5 3}(1), 160-179$.

Funke, M., Schularick, M. and Trebesch, C. (2016), 'Going to extremes: Politics after financial crises, 1870-2014', European Economic Review 88, 227-260.

Galor, O. and Moav, O. (2004), 'From Physical to Human Capital Accumulation: Inequality and the Process of Development', The Review of Economic Studies 71(4), 1001-1026. 
Galor, O. and Zeira, J. (1993), 'Income Distribution and Macroeconomics', The Review of Economic Studies 60(1), 35-52.

Gennaioli, N. and Tabellini, G. (2019), Identity, Beliefs, and Political Conflict. SSRN Electronic Journal 3300726 .

Gilens, M. (2005), 'Inequality and Democratic Responsiveness', Public Opinion Quarterly 69(5), 778796.

Gilens, M. (2012), Affluence and Influence: Economic Inequality and Political Power in America, Princeton University Press.

Giuliano, P. and Spilimbergo, A. (2009), Growing Up in a Recession: Beliefs and the Macroeconomy. NBER Working Paper, No. 15321, National Bureau of Economic Research.

Goldsmith, R. W. (1969), Financial Structure and Development. Yale University Press, New Haven, CT.

Granger, C. W. (1969), 'Investigating Causal Relations by Econometric Models and Cross-spectral Methods', Econometrica 37(3), 424-438.

Greenwood, J. and Jovanovic, B. (1990), 'Financial Development, Growth, and the Distribution of Income', Journal of Political Economy 98(5, Part 1), 1076-1107.

Haile, D., Sadrieh, A. and Verbon, H. A. (2008), 'Cross-racial envy and underinvestment in South African partnerships', Cambridge Journal of Economics 32(5), 703-724.

Hamilton, J. (1994), Time Series Analysis, first ed. Princeton University Press, Princeton.

Han, K. J. (2016), 'Income inequality and voting for radical right-wing parties', Electoral Studies $42,54-64$.

Han, S. M. and Chang, E. C. (2016), 'Economic inequality, winner-loser gap, and satisfaction with democracy', Electoral Studies 44, 85-97.

Hargreaves Heap, S. P. and Zizzo, D. J. (2009), 'The Value of Groups', American Economic Review 99(1), 295-323.

Hawkins, K. A. (2010), Venezuela's Chavismo and Populism in Comparative Perspective, Cambridge, Cambridge University Press.

Hellwig, T. and Samuels, D. (2007), 'Voting in Open Economies: The Electoral Consequences of Globalization', Comparative Political Studies 40(3), 283-306.

Hellwig, T. and Samuels, D. (2008), 'Electoral Accountability and the Variety of Democratic Regimes', British Journal of Political Science 38(1), 65-90. 
Hetherington, M. J. (2001), 'Resurgent Mass Partisanship: The Role of Elite Polarization', American Political Science Review 95(3), 619-631.

Hetherington, M. J. and Weiler, J. D. (2009), Authoritarianism and Polarization in American politics, Cambridge University Press.

Holtz-Eakin, D., Newey, W. and Rosen, H. S. (1988), 'Estimating Vector Autoregressions with Panel Data', Econometrica 56(6), 1371-1395.

Inglehart, R. and Norris, P. (2016), Trump, Brexit, and the Rise of Populism: Economic Have-Nots and Cultural Backlash, Boston, MA: Harvard Kennedy School.

International IDEA (2019), Political Finance Database, Accessed on 20 February 2019.

Iversen, T. and Soskice, D. (2015), 'Information, Inequality, and Mass Polarization: Ideology in Advanced Democracies', Comparative Political Studies 48(13), 1781-1813.

Jackman, R. W. and Volpert, K. (1996), 'Conditions Favouring Parties of the Extreme Right in Western Europe', British Journal of Political Science 26(4), 501-521.

Jeong, H. and Kim, S. (2018), 'Finance, Growth, and Inequality: New Evidence from the Panel VAR Perspective', Seoul Journal of Economics 31(2).

Jesuit, D. K., Paradowski, P. R. and Mahler, V. A. (2009), 'Electoral support for extreme right-wing parties: A sub-national analysis of Western European elections', Electoral Studies 28(2), 279-290.

Jordà, O., Schularick, M. and Taylor, A. (2017), Macrofinancial History and the New Business Cycle Facts, In NBER Macroeconomics Annual 2016, vol. 31, edited by Eichenbaum, M., and Parker, J.A. Chicago: University of Chicago Press.

Kaldor, N. (1957), 'A Model of Economic Growth', The Economic Journal 67(268), 591-624.

Kallis, G., Kostakis, V., Lange, S., Muraca, B., Paulson, S. and Schmelzer, M. (2018), 'Research On Degrowth', Annual Review of Environment and Resources 43, 291-316.

Kelley, J. and Evans, M. D. (1993), 'The Legitimation of Inequality: Occupational Earnings in Nine Nations', American Journal of Sociology 99(1), 75-125.

Kharas, H. (2010), The Emerging Middle Class in Developing Countries. OECD, Working Paper No. 285, OECD Development Centre.

King, G., Tanner, M. A. and Rosen, O. (2004), Ecological Inference: New Methodological Strategies, Cambridge: Cambridge University Press.

King, R. G. and Levine, R. (1993), 'Finance and growth: Schumpeter might be right', Quarterly Journal of Economics 108(3), 717-737. 
Knigge, P. (1998), 'The ecological correlates of right-wing extremism in Western Europe', European Journal of Political Research 34(2), 249-279.

Kramer, G. H. (1971), 'Short-Term Fluctuations in US Voting Behavior, 1896-1964', American Political Science Review 65(1), 131-143.

Krieger, T. and Meierrieks, D. (2019), 'Income inequality, redistribution and domestic terrorism', World Development 116, 125-136.

Kriesi, H. (2014), The Political Consequences of the Economic Crisis in Europe: Electoral Punishment and Popular Protest. In: N. Bermeo and L. M. Bartels (eds.), Mass Politics in Tough Times: Opinions, Votes and Protest in the Great Recession, Oxford: Oxford University Press, 297-333.

Kuznets, S. (1955), 'Economic Growth and Income Inequality', The American Economic Review $45(1), 1-28$.

Lachat, R. (2008), 'The impact of party polarization on ideological voting', Electoral Studies 27(4), 687-698.

Laeven, L. and Valencia, F. (2008), Systemic Banking Crises: A New Database, International Monetary Fund - Working Paper No. 8-224.

Laeven, L. and Valencia, F. (2012), Systemic Banking Crises Database: An Update., International Monetary Fund - Working Paper No. 12-163.

Levin, A., Lin, C.-F. and Chu, C.-S. J. (2002), 'Unit root tests in panel data: asymptotic and finitesample properties', Journal of Econometrics 108(1), 1-24.

Levine, R. (1997), 'Financial Development and Economic Growth: Views and Agenda', Journal of Economic Literature 35(2), 688-726.

Levine, R. (2005), 'Finance and Growth: Theory and Evidence', Handbook of Economic Growth 2005(1), 865-934.

Lewis-Beck, M. S. and Stegmaier, M. (2013), 'The VP-function revisited: a survey of the literature on vote and popularity functions after over 40 years', Public Choice 157(3-4), 367-385.

Li, H. and Zou, H.-f. (1998), 'Income Inequality Is Not Harmful for Growth: Theory and Evidence', Review of Development Economics 2(3), 318-334.

Lind, J. T. (2007), 'Fractionalization and the size of government', Journal of Public Economics 91(12), $51-76$.

Lubbers, M., Gijsberts, M. and Scheepers, P. (2002), 'Extreme right-wing voting in Western Europe', European Journal of Political Research 41(3), 345-378. 
Lubbers, M. and Scheepers, P. (2000), 'Individual and contextual characteristics of the German extreme Right-Wing vote in the 1990s. A test of complementary theories', European Journal of Political Research 38(1), 63-94.

Lubbers, M. and Scheepers, P. (2002), 'French front national voting: a micro and macro perspective', Ethnic and Racial Studies 25(1), 120-149.

Luttmer, E. F. (2001), 'Group Loyalty and the Taste for Redistribution', Journal of Political Economy $\mathbf{1 0 9}(3), 500-528$.

Majlesi, K., Dorn, D., Hanson, G. et al. (2020), 'Importing Political Polarization? The Electoral Consequences of Rising Trade Exposure', American Economic Review 110(10), 3139-83.

March, L. and Rommerskirchen, C. (2015), 'Out of left field? Explaining the variable electoral success of European radical left parties', Party Politics 21(1), 40-53.

McCarty, N. (2019), Polarization: What Everyone Needs to Know, Oxford: Oxford University Press.

McCarty, N., Poole, K. T. and Rosenthal, H. (2016), Polarized America: The Dance of Ideology and Unequal Riches, MIT Press.

McKinnon, R. I. (1973), Money and Capital in Economic Development, Brookings Institution, Washington, DC.

Meguid, B. M. (2005), 'Competition Between Unequals: The Role of Mainstream Party Strategy in Niche Party Success', American Political Science Review 99(3), 347-359.

Meltzer, A. H. and Richard, S. F. (1981), 'A Rational Theory of the Size of Government', Journal of Political Economy 89(5), 914-927.

Mian, A., Sufi, A. and Trebbi, F. (2014), 'Resolving Debt Overhang: Political Constraints in the Aftermath of Financial Crises', American Economic Journal: Macroeconomics 6(2), 1-28.

Milanovic, B. (2016), Global Inequality: A New Approach for the Age of Globalization, Cambridge: Harvard University Press.

Mudde, C. (2007), Populist radical right parties in Europe, Cambridge: University Press Cambridge.

Nannestad, P. and Paldam, M. (1994), 'The VP-function: A survey of the literature on vote and popularity functions after 25 years', Public Choice 79(3-4), 213-245.

OECD (2010), Tax Policy Reform and Economic Growth. OECD Tax Policy Studies, No. 20, Organisation for Economic Co-operation and Development.

OECD (2017), Housing (indicator), doi: 10.1787/63008438-en (accessed on 11 november 2017). 
O’Grady, T. (2019), 'Careerists Versus Coal-Miners: Welfare Reforms and the Substantive Representation of Social Groups in the British Labour Party', Comparative Political Studies 52(4), 544-578.

Okun, A. M. (2015), Equality and efficiency: The big tradeoff, Brookings Institution Press.

Ostry, M. J. D., Berg, M. A. and Tsangarides, M. C. G. (2014), Redistribution, inequality, and growth, International Monetary Fund.

Owyang, M. and Shell, H. (2016), Taking Stock: Income Inequality and the Stock Market. Economic Synopses, Federal Reserve Bank of St. Louis 7, 1-2.

Perotti, R. (1996), 'Growth, income distribution, and democracy: What the data say', Journal of Economic growth 1(2), 149-187.

Persson, T. and Tabellini, G. (1994), 'Is Inequality Harmful for Growth?', The American Economic Review 84(3), 600-621.

Piketty, T. (2014a), 'Capital in the 21st Century', Cambridge: The Belknap Press of Harvard University Press. .

Piketty, T. (2014b), Capital in the $21^{\text {st }}$ Century, Cambridge Massachusetts: The Belknap Press of Harvard University Press.

Pontusson, J. and Rueda, D. (2010), 'The Politics of Inequality: Voter Mobilization and Left Parties in Advanced Industrial States', Comparative Political Studies 43(6), 675-705.

Powell, G. B., Whitten, G. D. et al. (1993), 'A Cross-National Analysis of Economic Voting: Taking Account of the Political Context', American Journal of Political Science 37(2), 391-414.

Rattinger, H. (1981), Unemployment and the 1976 election in Germany: Some findings at the aggregate and the individual level of analysis. In: Hibbs, D. \& Fassbender, H. (eds.), Contemporary Political Economy. Amsterdam: North-Holland, 121-135.

Rioja, F. and Valev, N. (2004), 'Finance and the Sources of Growth at Various Stages of Economic Development', Economic Inquiry 42(1), 127-140.

Roodman, D. (2009), 'How to do Xtabond2: An introduction to Difference and System GMM in Stata', The Stata Journal 9(1), 86-136.

Rooduijn, M. and Burgoon, B. (2018), 'The Paradox of Well-being: Do Unfavorable Socioeconomic and Sociocultural Contexts Deepen or Dampen Radical Left and Right Voting Among the Less Well-Off?', Comparative Political Studies 51(13), 1720-1753.

Rydgren, J. (2008), 'Immigration sceptics, xenophobes or racists? Radical right-wing voting in six West European countries', European Journal of Political Research 47(6), 737-765. 
Rydgren, J. (2012), Class politics and the radical right, London: Routledge.

Sahay, R., Čihák, M., N'diaye, P., Barajas, A., Bi, R., Ayala, D., Gao, Y., Kyobe, A., Nguyen, L., Saborowski, C., Svirydzenka, K. and Reza, S. (2015), 'Rethinking Financial Deepening: Stability and Growth in Emerging Markets', IMF Staff Discussion Note 15(08).

Saint-Paul, G. and Verdier, T. (1993), 'Education, democracy and growth', Journal of Development Economics 42(2), 399-407.

Scharpf, F. W. (1999), Governing in Europe: Effective and democratic?, Oxford University Press.

Schröder, M., Saßenroth, D., Körtner, J., Kroh, M. and Schupp, J. (2013), Experimental Evidence of the Effect of Monetary Incentives on Cross-Sectional and Longitudinal Response: Experiences from the Socio-Economic Panel (SOEP). SOEPpapers on Multidisciplinary Panel Data Research, No. 603, Deutsches Institut für Wirtschaftsforschung (DIW), Berlin.

Schumpeter, J. A. (1912), Theorie der Wirtschaftlichen Entwicklung [The Theory of Economic Development]. Leipzig: Dunker \& Humblot; trans. Redvers Opie, Cambridge, MA:Hardvard University Press, 1934.

Senik, C., Stichnoth, H. and Van der Straeten, K. (2009), 'Immigration and Natives' Attitudes towards the Welfare State: Evidence from the European Social Survey', Social Indicators Research 91(3), 345-370.

Shaw, E. (1973), Financial Deepening in Economic Development. Oxford University Press, New York.

Sims, C. A. (1980), 'Macroeconomics and Reality', Econometrica 48(1), 1-48.

Singer, E., Van Hoewyk, J., Gebler, N. and McGonagle, K. (1999), 'The Effect of Incentives on Response Rates in Interviewer-Mediated Surveys', Journal of Official Statistics 15(2), 217-230.

Solt, F. (2009), 'Standardizing the World Income Inequality Database', Social Science Quarterly $\mathbf{9 0}(2), 231-242$.

Solt, F. (2010), 'Does Economic Inequality Depress Electoral Participation? Testing the Schattschneider Hypothesis', Political Behavior 32(2), 285-301.

Solt, F. (2016), 'The Standardized World Income Inequality Database', Social Science Quarterly 97(5), 1267-1281.

Solt, F. (2019), The Standardized World Income Inequality Database, Version 8-9. Harvard Dataverse, Available at: https://doi.org/10.7910/DVN/LM4OWF

Stichnoth, H. and Van der Straeten, K. (2013), 'Ethnic Diversity, Public Spending, and Individual Support for the Welfare State: A Review of the Empirical Literature', Journal of Economic Surveys 27(2), 364-389. 
Stiglitz, J. E. (2000), 'Capital Market Liberalization, Economic Growth, and Instability', World development 28(6), 1075-1086.

Stiglitz, J. E. (2013), The price of inequality: How today's divided society endangers our future, New York: W.W. Norton \& Company.

Stimson, J. (2018), Public Opinion In America: Moods, Cycles, and Swings (Second Edition), London: Routledge.

Sumption, M. and Vargas-Silva, C. (2020), Net migration to the UK. The Migration Observatory, University of Oxford, UK.

Svirydzenka, K. (2016), Introducing a New Broad-based Index of Financial Fevelopment. IMF Working Paper No. 16/5, International Monetary Fund.

Swank, D. and Betz, H.-G. (2003), 'Globalization, the welfare state and right-wing populism in Western Europe', Socio-Economic Review 1(2), 215-245.

Tabellini, M. (2020), 'Gifts of the Immigrants, Woes of the Natives: Lessons from the Age of Mass Migration', The Review of Economic Studies 87(1), 454-486.

Tafjel, H. and Turner, J. C. (1979), An Integrative Theory of Intergroup Conflict. In: Worchel S. and Austin W (eds.), The Social Psychology of Intergroup Relations, Monterey, CA: Brooks/Cole, $3-47$.

Tafjel, H. and Turner, J. C. (1986), The Social Identity Theory of Intergroup Behavior. In: Worchel S. and Austin W (eds.), The Psychology of Intergroup Relations, Chicago: Nelson-Hall, 7-24.

Thirlwall, A. P. (1994), Growth And Development: With Special Reference To Developing: with Special Reference to Developing Economies, Macmillan International Higher Education.

Ura, J. D. and Ellis, C. R. (2012), 'Partisan Moods: Polarization and the Dynamics of Mass Party Preferences', The Journal of Politics 74(1), 277-291.

Van Kesteren, F., Altaf, A. and de Weerd, R. (2019), Trickle Up: how pro-poor investments drive economic development. The Broker, Synthesis Study.

Visser, M., Lubbers, M., Kraaykamp, G. and Jaspers, E. (2014), 'Support for radical left ideologies in Europe', European Journal of Political Research 53(3), 541-558.

Vlaicu, R. (2018), 'Inequality, participation, and polarization', Social Choice and Welfare 50(4), 597624 .

Voorheis, J., McCarty, N. and Shor, B. (2015), Unequal Incomes, Ideology and Gridlock: How Rising Inequality Increases Political Polarization, Available at SSRN: https://ssrn. com/abstract= 2649215 
Wagner, M. (2012), 'When do parties emphasise extreme positions? How strategic incentives for policy differentiation influence issue importance', European Journal of Political Research 51(1), 64-88.

Werts, H., Scheepers, P. and Lubbers, M. (2013), 'Euro-scepticism and radical right-wing voting in Europe, 2002-2008: Social cleavages, socio-political attitudes and contextual characteristics determining voting for the radical right', European Union Politics 14(2), 183-205.

World Bank (2017), World Development Indicators, Variables: Inflation, GDP deflator (annual \%), General government final consumption expenditure (\% of GDP), (Accessed on 20 December 2017). Available at https://data.worldbank.org/products/wdi

World Bank (2019), World Development Indicators, Variables: Income share held by highest 10\%, Income share held by highest $20 \%$, Income share held by lowest $10 \%$, Income share held by lowest 20\%, (Accessed on 15 November 2019). Available at https://data.worldbank.org/. 